\title{
Bone Mass Preservation and Fracture Risk Assessment with Bisphosphonate Therapy During Spaceflight
}

\author{
A Thesis \\ Presented to the Faculty of \\ California Polytechnic State University \\ San Luis Obispo \\ In Partial Fulfillment of the \\ Requirements for the Degree \\ Master of Science in Engineering \\ with a Specialization in Biomedical Engineering
}

by

Christopher Gardina

June 2008 
AUTHORIZATION FOR REPRODUCTION OF MASTER'S THESIS

I grant permission for the reproduction of this thesis in its entirety or any of its parts, without further authorization from me.

Christopher $R$ thad ic

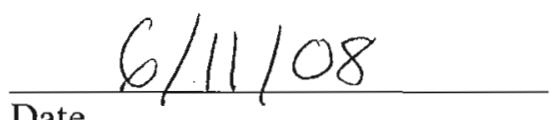

ii 


\section{APPROVAL}

Title: Bone Mass Preservation and Fracture Risk Assessment with Bisphosphonate Therapy During Spaceflight

Author: $\quad$ Christopher Gardina

Date Submitted: June 2008

Dr. Scott Hazelwood Committee Chair

Dr. Lanny Griffin

Committee Member

Dr. Peter Schuster

Committee Member
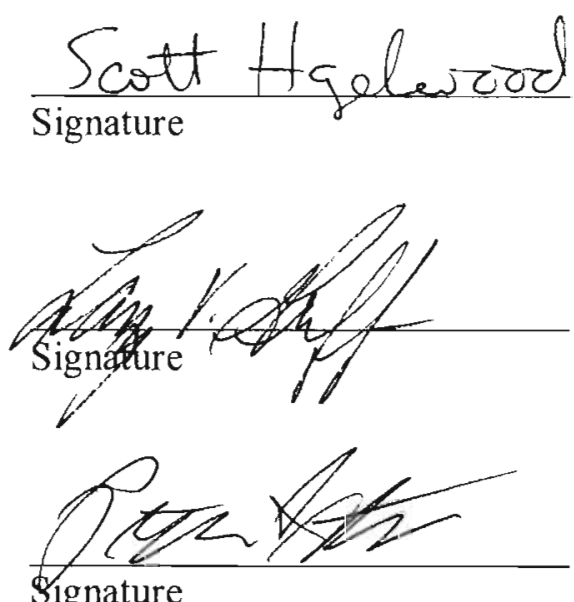


\begin{abstract}
Bone Mass Preservation and Fracture Risk Assessment with Bisphosphonate Therapy During Spaceflight

Christopher Gardina
\end{abstract}

Space exploration and microgravity have substantial negative effects on the human body. Symptoms of space explorers include cardiovascular deconditioning, bone loss, muscular atrophy, and impairment of neurovestibular and sensory function. The great loss of bone due long-duration spaceflight increases fracture risk, jeopardizing the success of the mission and postflight recovery. Bisphosphonates may be able to counteract this bone loss by altering the remodeling process. These drugs increase bone mass, thus reducing fracture risk, but also lead to increased levels of fatigue microdamage. Fracture risk can be lowered by increasing both bone mass (quantity) and bone quality.

The purpose of this study was to create a computer model to simulate bisphosphonate treatment on astronauts while traveling in space in order to examine the ability of bisphosphonates to maintain bone mass in a microgravity environment and reduce fracture risk of bone upon return to Earth. Various bisphosphonate treatment potencies and bone balance ratios given at different time points (either at or before spaceflight) were examined. Flight duration was also varied to examine short-term (10 days) to long-term (1 year) effects of microgravity on bone mineral density (BMD), a measure commonly used to estimate bone strength, and damage accumulation. The model predicted bisphosphonate treatments with low to intermediate suppression of remodeling activation and that create higher bone balance ratios cause reductions in fracture risk. The simulation also predicted significant changes to BMD and damage upon return to Earth 
as the remodeling response readjusted to higher stress conditions. For treatments highly suppressing remodeling activation, these predicted post flight changes included decreased $\mathrm{BMD}$ and increased damage accumulation. Low levels of remodeling suppression led the model to predict substantial increases in BMD and small increases in damage postflight. Postflight changes were minimal for treatments with intermediate suppression. 


\section{ACKNOWLEDGEMENTS}

I would like to thank all of those who have assisted me with this project, either through advice, example, or support. A great deal of my gratitude goes to my advisor, Dr. Scott Hazelwood, who developed the idea and guided and inspired me on numerous occasions. Lastly, I would like to thank my parents who gave me love and support throughout my life and provided me the opportunity to pursue higher education. 


\section{TABLE OF CONTENTS}

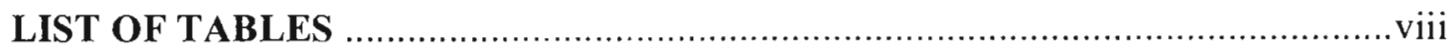

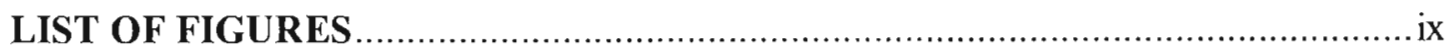

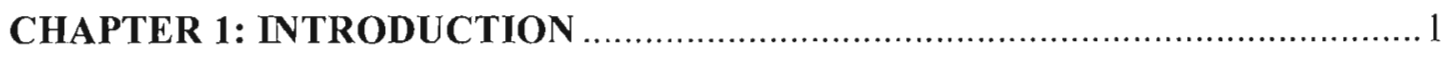

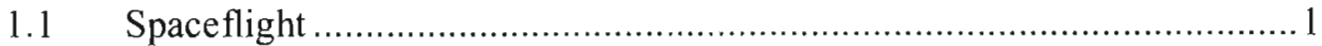

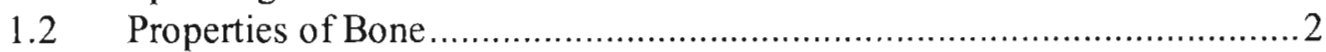

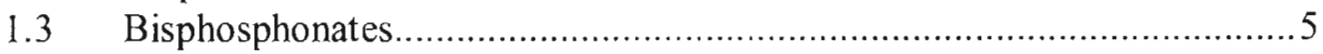

$1.4 \quad$ Previous Models...................................................................... 7

1.5 Simulating Bisphosphonate Treatment during Spaceflight .................... 11

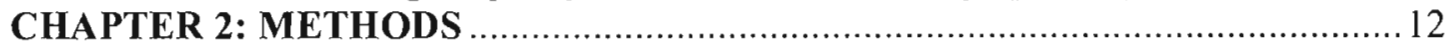

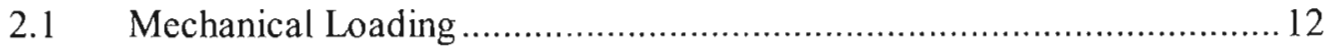

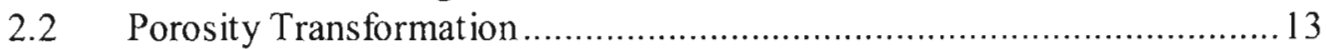

2.3 BMU Activation..................................................................... 14

2.4 Microdamage Accumulation...................................................... 15

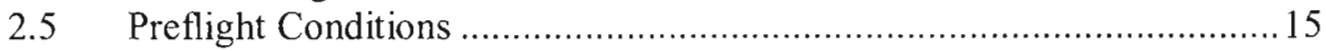

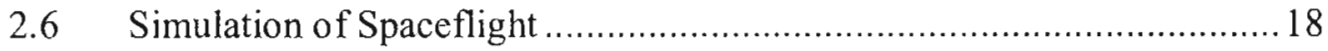

2.7 Simulation of Bisphosphonates................................................ 18

$2.8 \quad$ Simulation of Return to Earth ........................................................ 20

2.9 Model Implementation .......................................................... 20

2.10 Postflight Parameter Analysis ....................................................... 21

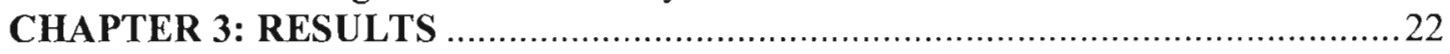

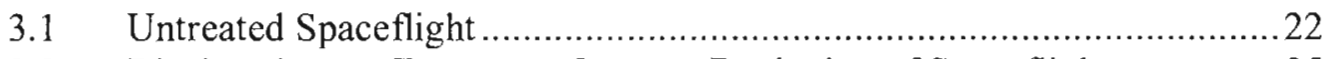

3.2 Bisphosphonate Treatment Onset at Beginning of Spaceflight ...............25

$3.3 \quad$ Preflight Bisphosphonate Treatment ............................................. 28

3.4 Varying Onset of Bisphosphonate Treatment for Spaceflight.................. 32

3.5 One-Year Postflight, Posttreatment Recovery ..................................... 33

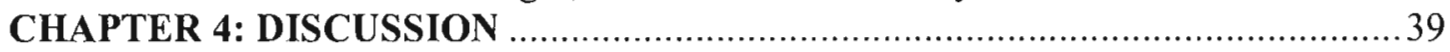

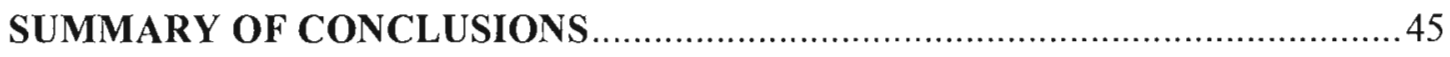

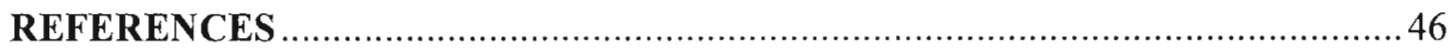

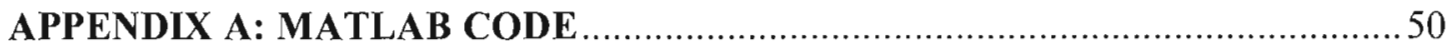

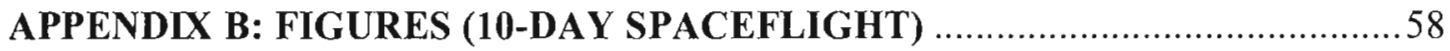

APPENDIX C: FIGURES (90-DAY SPACEFLIGHT) …............................. 70

APPENDIX D: FIGURES (180-DAY SPACEFLIGHT) ................................ 82

APPENDIX E: FIGURES (365-DAY SPACEFLIGHT) .................................. 95 


\section{LIST OF TABLES}

Table 2.1. Model parameters with values obtained from simulating remodeling in trabecular bone before entering space and at the end of a 180-day space simulation.

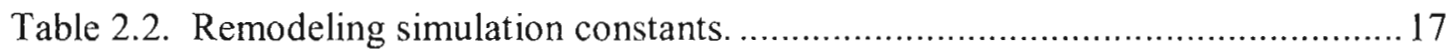

Table 2.3. Bisphosphonate effects analyzed during the simulation. .............................. 19

Table 3.1. Ratio of percent preflight increase of damage (D) to BMD ........................... 32 


\section{LIST OF FIGURES}

Figure 1.1. Illustration of long bone showing cortical and trabecular bone.................... 3

Figure 1.2. A healthy trabecular bone strut and one with microdamage........................ 3

Figure 1.3. A BMU containing osteoblasts and osteoclasts. .................................... 4

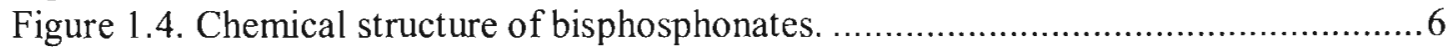

Figure 1.5. Schematic of a bone remodeling algorithm by Hazelwood et al.................... 9

Figure 1.6. Schematic of a bone remodeling algorithm by Hernandez et al. ................. 10

Figure 2.1. Trabecular volume and cross-section. .................................................. 17

Figure 2.2. Bisphosphonate potency as a function of number of resorbing BMUs......... 19

Figure 3.1. Predicted percent decreases in BMD and damage (D) of untreated bone

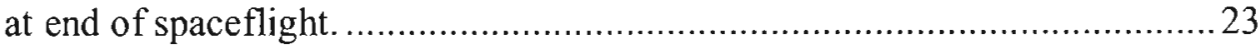

Figure 3.2. Predicted effects of 180-day spaceflight on BMD of untreated bone from beginning of flight through 19.5 years postflight. ................................... 23

Figure 3.3. Predicted effects of 180-day spaceflight on damage accumulation (D) of untreated bone from beginning of flight through 19.5 years postflight.........24

Figure 3.4. Predicted percent changes in BMD and damage (D) at end of 10-day

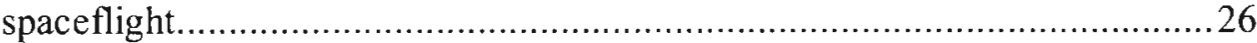

Figure 3.5. Predicted percent changes in BMD and damage (D) at end of 90-day spaceflight

Figure 3.6. Predicted percent changes in BMD and damage (D) at end of 180-day spaceflight

Figure 3.7. Predicted percent changes in BMD and damage (D) at end of 365-day spaceflight.

Figure 3.8. Predicted preflight increase in BMD and damage (D) due to 7-day preflight treatment.

Figure 3.9. Predicted preflight increase in BMD and damage (D) due to 14-day preflight treatment.

Figure 3.10. Predicted preflight increase in BMD and damage (D) due to 30-day preflight treatment.

Figure 3.11. Predicted preflight increase in BMD and damage (D) due to 90-day preflight treatment.

Figure 3.12. Predicted preflight increase in BMD and damage (D) due to 180-day preflight treatment.

Figure 3.13. Predicted percent changes in BMD and damage (D) at end of 365-day spaceflight due to 30 -day preflight treatment.

Figure 3.14. Predicted percent changes in BMD and damage (D) at end of 365-day spaceflight due to 180-day preflight treatment.

Figure 3.15. Predicted bisphosphonate effects on BMD and posttreatment return to Earth from 180-day spaceflight. ........................................................... 34

Figure 3.16. Predicted bisphosphonate effects on BMD and posttreatment return to Earth from 180-day spaceflight.

Figure 3.17. Predicted bisphosphonate effects on damage accumulation (D) and posttreatment return to Earth from 180-day spaceflight. 36

Figure 3.18. Predicted bisphosphonate effects on damage accumulation (D) and posttreatment return to Earth from 180-day spaceflight. 
Figure 3.19. Predicted bisphosphonate effects on damage accumulation (D) and posttreatment return to Earth from 365-day spaceflight.

Figure 3.20. Predicted bisphosphonate effects bcginning 90 days preflight on BMD and posttreatment return to Earth from 180-day spaceflight

Figure 3.21. Predicted bisphosphonate effects beginning 90 days preflight on damage accumulation (D) and posttreatment return to Earth from 180-day spaceflight. 


\section{CHAPTER 1: INTRODUCTION}

\subsection{Spaceflight}

Microgravity has many adverse cffects on the human body that hinder the ability of astronauts to explore outer space. The physiologic adaptations caused by unloading include cardiovascular deconditioning, bone loss, muscular atrophy, and impairment of neurovestibular and sensory function [1]. The focus here is on bone loss, which results from the reduced levels of stress caused by weightlessness. This loss poses a significant health risk for astronauts and is a major deciding factor of mission duration. In 6-month missions to the International Space Station (ISS), astronauts experienced up to 5 percent loss of bone mineral density (BMD) in the lumbar spine and 10 percent in the proximal femur [2]. On the MIR space station, the greatest bone loss observed in a crew member was on the order of half the mineral loss incurred in a lifetime of normal aging [3]. Upon return to Earth's gravitational environment, the average fracture risk for a space explorer paralleled the estimated level for 70- to 80 -year old postmenopausal women [4]. While bone has the ability to recovery mineral, it is much slower than the rate at which it is lost. Complete recovery may take from 1 to 3 years, and in many cases bone will never fully recover the mineral lost during spaceflight [5].

NASA and other space exploration agencies have developed exercise programs to combat bone loss in space. In earlier space missions, exercise routines utilized bungee cords for resistive exercises, and stationary bicycles and treadmills for longer, aerobic exercises. More recent missions to the ISS combined these older techniques with a new piece of equipment, the Interim Resistive Exercise Device (iRED), focusing on resistive training. This apparatus, by sufficiently increasing loading intensity, may lead to shorter, 
more effective exercise routines [5]. Thus far, exercise routines have been able to slow bone loss, but remain unsuccessful at completely preventing it from occurring. NASA believes that coupling pharmacological treatments with their exercise programs will allow them to reach their goal [4]. Though the efficacy of many treatments such as testosterone, parathyroid hormone, calcium, vitamin $\mathrm{D}$, and vitamin $\mathrm{K}$, are being investigated, bisphosphonates seem a likely candidate [6]. Bisphosphonate therapy has the potential to increase bone mass in space just as it does for osteoporotic patients on Earth [2], though the pharmacokinetic altering effects of spaceflight have yet to be determined.

\subsection{Properties of Bone}

In a healthy individual, bones provide structure and support, and with the help of muscles, tendons and ligaments, they allow for movement of the body. There are two distinct types of bone tissue: cortical or compact bone and trabecular (also referred to as cancellous or spongy) bone. The main difference between these two types is their porosity. Cortical bone is fairly dense and non-porous, while trabecular bone has a much higher porosity and is made up of 'struts' that form an interconnected matrix (Figures 1.1 and 1.2). Theoretically, the porosity of bone tissue can be anywhere from 0 to 100 percent; however, it is almost always either very high (trabecular bone) or very low (cortical bone) and rarely in the intermediate range [7]. Cortical bone contains cylindrical units called osteons (Figure 1.1) and typically has a porosity of 5 to 10 percent. It can be found in the shafts and outermost layer of bone. Trabecular bone, normally 75 to 95 percent porous, is contained deep inside the bone and is filled with marrow [7]. It is made up of a matrix of packets of bone. 


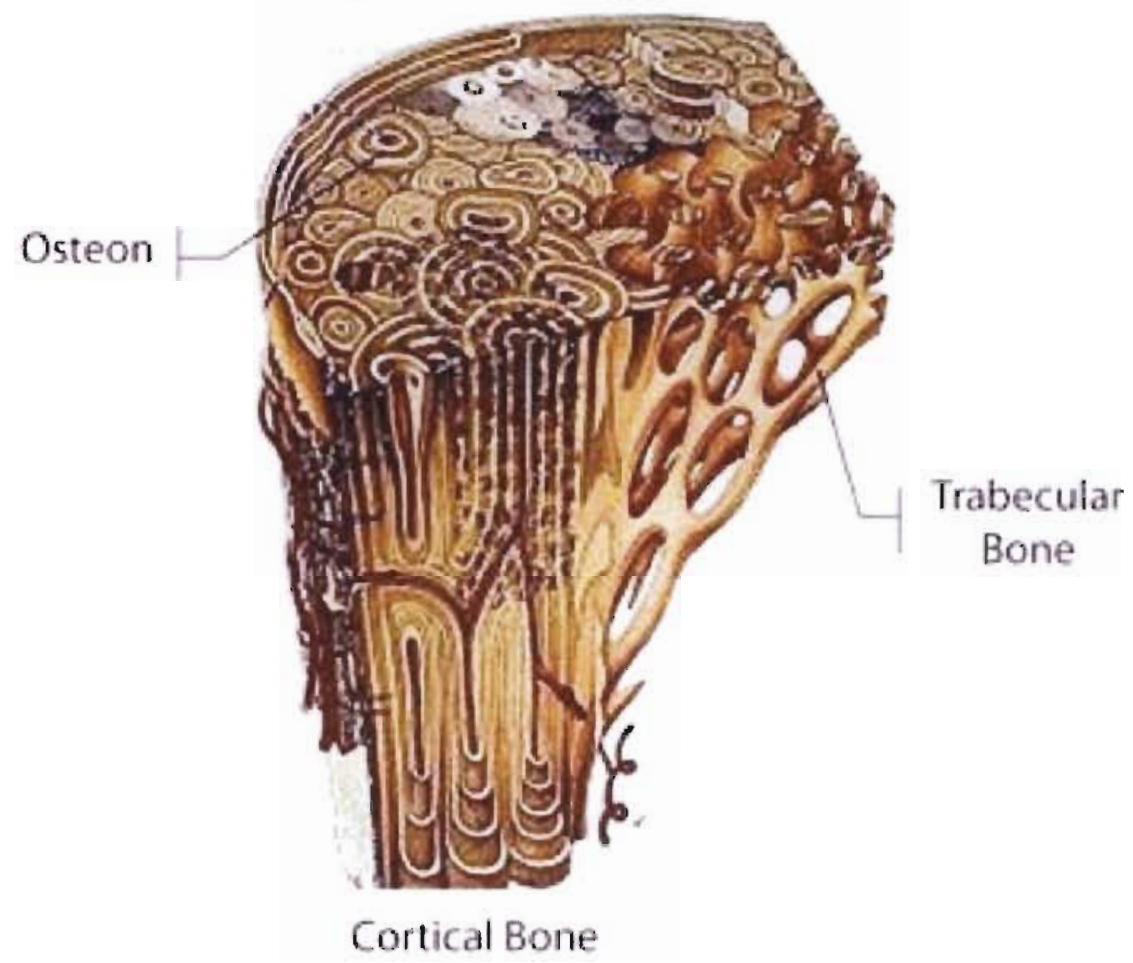

Figure 1.1. Illustration of long bone showing cortical and trabecular bone [8].
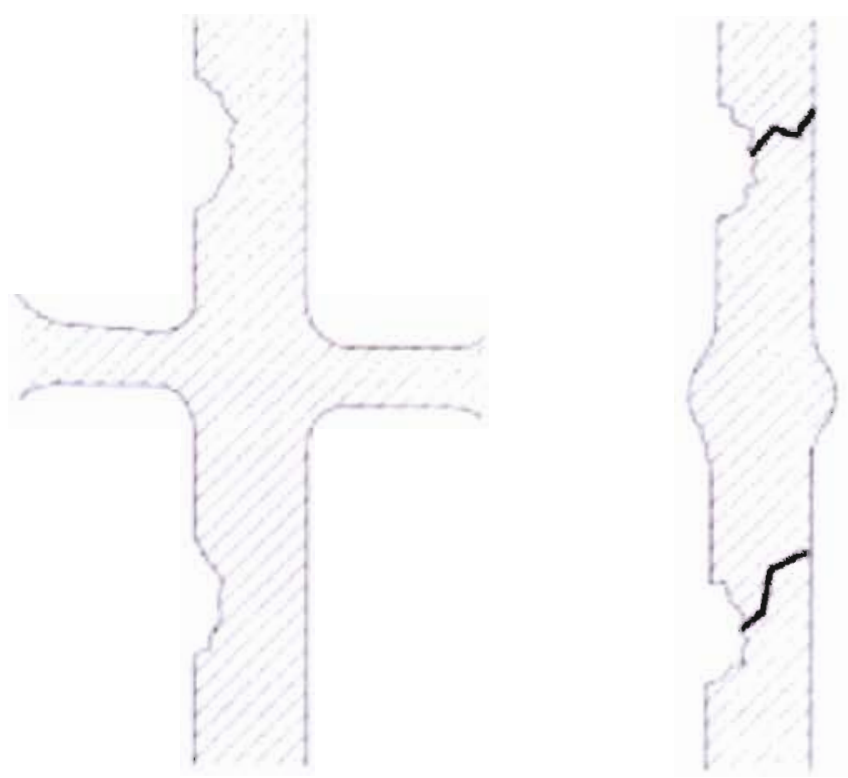

Figure 1.2. A healthy trabecular bone strut and one with microdamage [9]. 
Bone tissue experiences repetitive stress, leading to the formation of microdamage. Remodeling removes this damage, preventing fatigue failure from occurring under normal conditions [10]. Bone is made up of many packets of bone cells and minerals that are constantly undergoing this process. There are three major cells that partake in remodeling: osteoblasts, osteoclasts, and osteocytes. Osteoblasts and osteoclasts are bone-forming and bone-resorbing cells, respectively. Osteocytes are differentiated osteoblasts that are now fused into the bone matrix. Their role is to sense mechanical stimuli and send signals based on what they sensed to the surrounding bone cells [7]. The 3 to 4 month process of remodeling begins when osteoclasts receive signals to resorb damaged or fatigued bone. Osteoblasts then take over to remodel and 'fill in' the trenches created by the osteoclasts [7]. Together, osteoblasts and osteoclasts make up basic multicellular units (BMUs) of bone (Figure 1.3).

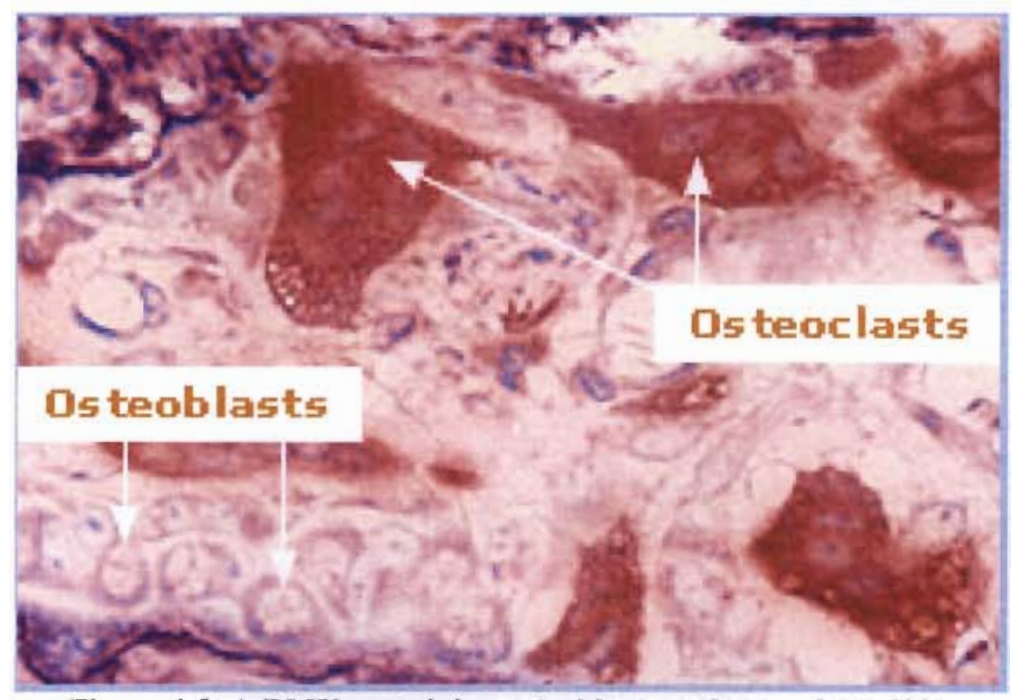

Figure 1.3. A BMU containing osteoblasts and osteoclasts [11].

BMUs are activated to remodel not only in response to increased microdamage $[12,13,14,15]$, but also due to disuse situations where bone use is reduced $[16,17]$. 
Exposing bone to fewer loading cycles or reduced load lowers the frequency or magnitude of strain experienced by the bone so that its current level of strength is no longer necessary. Bone is largely remodeled in order to minimize bone mass while maintaining the strength necessary to support the body [18]. The equilibrium point, where remodeling due to mechanical loading is such that bone resorption equals bone formation, varies from subject to subject. In general, as applied force and strain increase, the number of cycles required to maintain bone mass decreases [19].

\subsection{Bisphosphonates}

Bisphosphonates provide new hope for long-duration space exploration. This class of drugs treats diseases with elevated bone remodeling by suppressing osteoclastic function [18]. Bisphosphonates suppress resorption upon physical contact with osteoclasts [20]. They have a high affinity for bone mineral [21] and bind to bone in areas where resorption has exposed hydoxyapatite [22]. There are a variety of bisphosphonates, each having different potency and cellular mechanisms inhibiting BMU activation [23]. The various potencies allow for a range in the degree of resorption suppression [24].

Bisphosphonates vary due to their chemical composition (Figure 1.4). First generation bisphosphonates, etidronate, clodronate, and tiludronate, did not have side chains containing nitrogen nor hydroxyl groups. These first bisphosphonates were less potent and did not specifically target bone as well as those from generation two [25]. Second generation bisphosphonates, including alendronate, pamidronate, and risedronate, each contain a hydroxyl group on the $\mathrm{R}_{1}$ side chain and have a chain containing nitrogen on $\mathrm{R}_{2}$. The hydroxyl group on the carbon atom causes the increased affinity for bone and the $R_{2}$ chain determines the potency and mode of action of the drug [25]. The P-C-P 
chemical substructure enhances the safety and efficacy of the compound by increasing the affinity for calcium and resisting the metabolic processes of the body [25]. Although newer bisphosphonates have a very high affinity for mineral, only about half of any dose reaches bone [25]. Once they do reach bone and bind to exposed mineral, they can be uptaken by osteoclasts. While bound to osteoclasts, they exert their inhibiting effect by interfering with enzymatic activity.

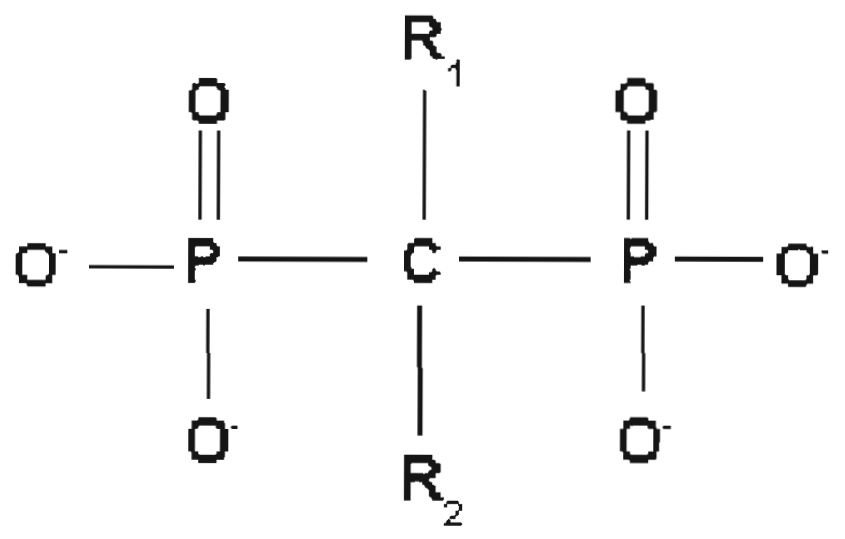

Figure 1.4. Chemical structure of bisphosphonates.

Bisphosphonates increase bone mass by reducing the temporary porosity created by remodeling known as the remodeling space, and by creating a positive bone balance where more bone is added than is removed $[23,26]$. Experimental data exists depicting the resulting bone mass increase in patients with osteoporosis. Spinal BMD increased 8.8 percent and reduced vertebral fractures by 48 percent after 3 years of alendronate treatment [27]. Another study reported increased spinal BMD throughout 7 years of alendronate treatment on postmenopausal women [28].

Bisphosphonates also can have negative effects on bone. Lowered remodeling levels increase microdamage accumulation and reduce toughness because less damage is removed [23]. Mineralization of the bones also increases, leading to brittler bones that 
are less resistant to crack propagation. One-year studies of alendronate and risedronate treatment on dogs showed microdamage accumulation and BMU activation frequency to be inversely proportional [29]. The overall goal of these therapies is to minimize the amount of damage accumulated while increasing bone mass. In the long run, high potency bisphosphonate treatment may in fact be detrimental if bone quality degrades enough to increase fracture risk due to high amounts of microdamage. Long-term effects of bisphosphonates on bone remodeling still remain unclear. Since studies can take up to 10 years to acquire real data, mathematically modeling the data and relationships obtained from shorter studies may be a more effective method of gathering insight into these phenomena.

\subsection{Previous Models}

Computational models are commonly used to test theories regarding the adaptation of bone to mechanical and physiological stimuli. Early models tested bone's mechanical adaptation. Carter et al. [30] and Huiskes et al. [31] were the first to use finite element modeling to develop mathematical relationships between mechanical loading and trabecular bone density [7]. In their, model, Carter et al. [30] focused on a daily mechanical stimulus based on stress and an error function. The error function tracked the difference between the stress stimulus at a given time point and a predetermined equilibrium stress stimulus. The apparent density of each element in the model was adjusted according to the error function. New modulus values were calculated by multiplying the changes in density by a constant. These new values were used at the next time point to recalculate the daily stress stimulus for each element. 
Huiskes' approach to model bone [31] has been shown to be the equivalent of Carter's but with different coefficients [7]. The model invoked bone adaptation by using strain energy density as the mechanical stimulus. New modulus values were calculated from strain energy density as in Carter's model [30] described above. The purpose of this particular model was to predict and analyze changes in bone due to total hip arthroplasties. Though both of these models were sufficient at simulating the mechanical environment that bone is subjected to, they did not include adaptations due to cellular responses.

A model by Hazelwood et al. [18] incorporates responses to both mechanical and biological stimuli. The model takes into account the cellular responses of BMUs. It predicts changes in porosity and elastic modulus based on simulated responses to mechanical stimuli such as disuse or overload and to the biological stimulus of damage accumulation. The schematic below (Figure 1.5) shows the basis for the simulation. Note that damage and disuse affect BMU activation frequency, which in turn affects porosity and modulus. The schematic shows remodeling to be a dynamic loop. 


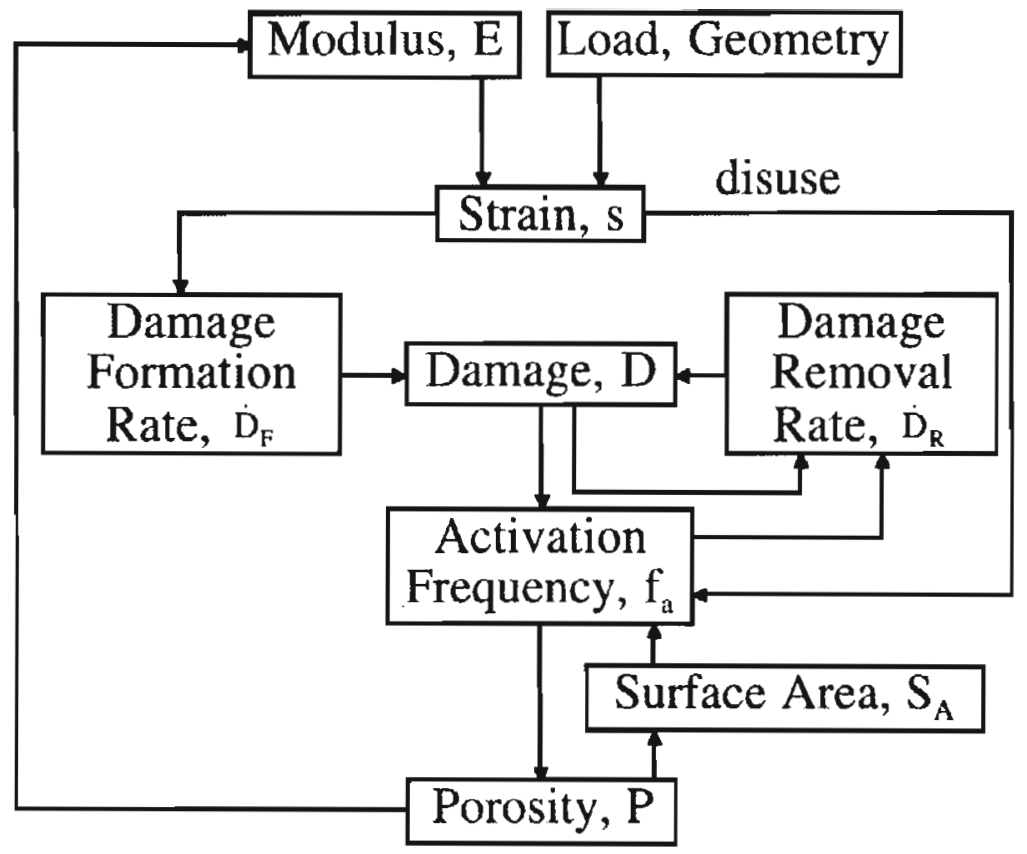

Figure 1.5. Schematic of a bone remodeling algorithm by Hazelwood et al. [18].

In early computational models simulating bisphosphonate effects, the basic strategy was to match clinical results of the treatment. The models simulated varying parameters such as BMU activation frequency, resorption and formation periods, bone balance, and mineralization $[32,33,34,23]$, but failed to include other stimuli important to long-term changes to BMD. These models did not take into account bone remodeling due to mechanical loading nor microdamage accumulation. Heaney et al. [32] developed a model that was fairly accurate when predicting bisphosphonate effects on BMD out to 6 months. The model simulated treatment using the bone balance method of decreasing bone turnover, decreasing remodeling space, increasing focal bone balance, and keeping bone mineralization constant. Hernandez et al. [33] developed a model to compare this method with the mineralization method in which remodeling space is decreased, focal bone balance remains the same, and bone mineralization is varied. This model included a longer secondary mineralization period to account for increases to BMD in the long term. 
The schematic shown in Figure 1.6 shows the lack of mechanical stimuli in the model developed by Hernandez et al. [33]. Lacy et al. [34] developed a model of bisphosphonate treatment in which various parameters were closely analyzed. Activation frequency was found to have the greatest influence on predicting changes in bone volume [34].

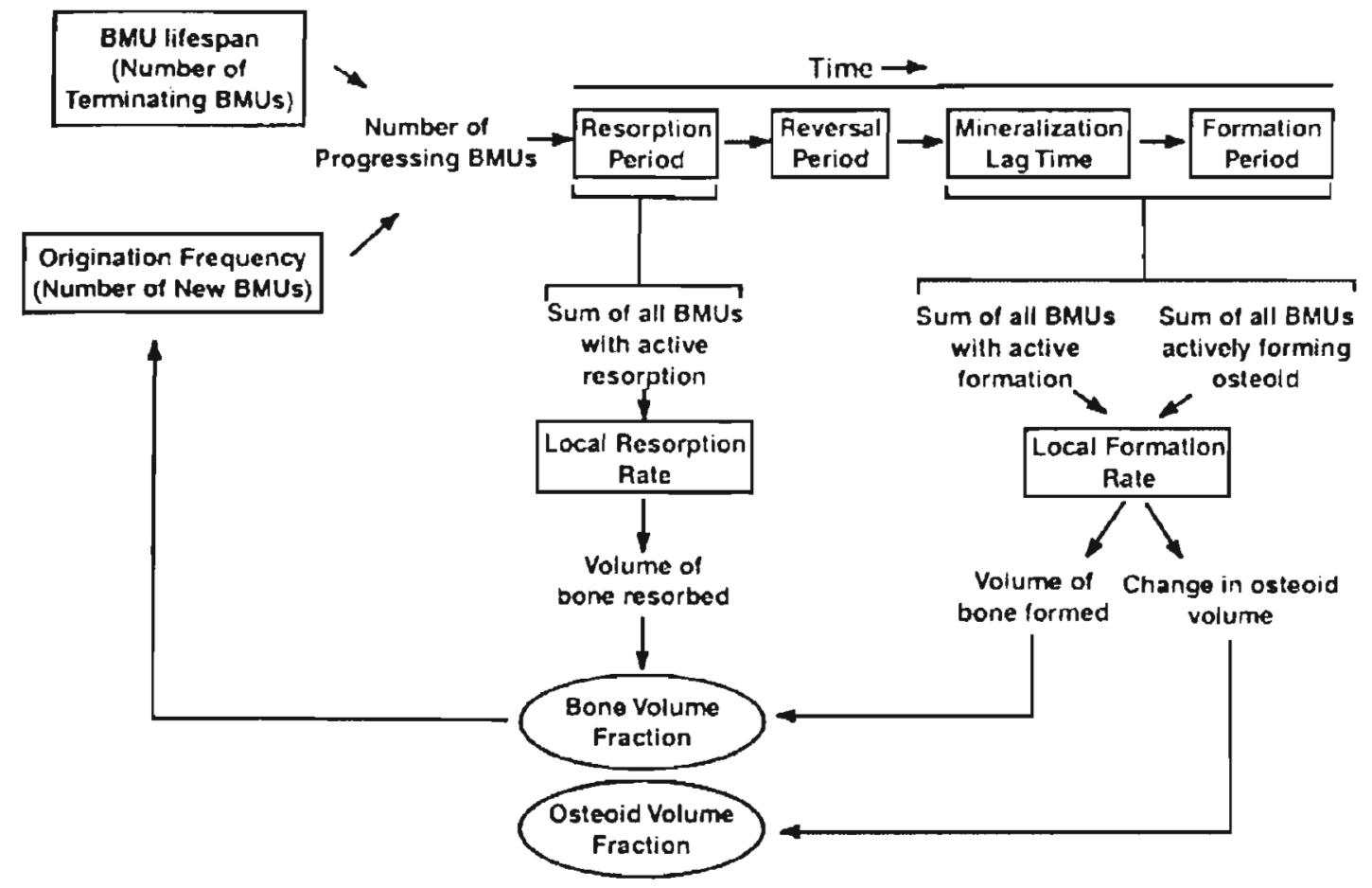

Figure 1.6. Schematic of a bone remodeling algorithm by Hernandez et al. [33].

A model developed by Nyman et al. [23] utilized both mechanisms of remodeling described previously, in response to mechanical loading and damage accumulation, to simulate the long-term effects of bisphosphonate usage. The model was designed for examining postmenopausal osteoporosis, so it also included a bone remodeling response to estrogen deficiency. The results of the model showed that the reduction of resorption space caused by bisphosphonate treatment played a key role in increasing bone volume over the long run. Also, Nyman et al. [23] concluded that the disuse response contributed 
to the eventual plateau of bone gain that other models failed to achieve [32,33]. Overall, the model showed incomplete suppression of BMU activation frequency and positive bone balances to permanently increase bone volume with minor gains in microdamage accumulation [23]. Various aspects of this model are implemented in this study and will be covered in more detail in the next section and in the methods chapter.

\subsection{Simulating Bisphosphonate Treatment during Spaceflight}

The goal of this study is to create a computer model to simulate bisphosphonate treatment on astronauts while traveling in space in order to examine bisphosphonates' ability to maintain bone mass in a microgravity environment and reduce fracture risk of bone upon return to Earth. We will examine various bisphosphonate treatment potencies and bone balance ratios given at different time points (either at or before spaceflight). Flight duration will also be varied to examine short-term (10 days) to long-term (1 year) effects of microgravity on BMD and damage accumulation. To examine these effects, mathematical relationships modeling bisphosphonate therapies [23] were applied to a computational model developed by Hazelwood et al [18] that was modified to investigate the influence of microgravity on the bone remodeling process.

This simulation will not only increase the understanding of the effects of spaceflight and bisphosphonate usage, but it may also lead to advances of other proposed treatments such as parathyroid hormone, testosterone, and vitamin $\mathrm{K}_{2}$ [6]. Understanding each therapy will eventually lead to better treatments on Earth and in space, decrease the health risks of space travel, and drastically expand the bounds of human space exploration. 


\section{CHAPTER 2: METHODS}

\subsection{Mechanical Loading}

This model simulates cyclic uniaxial loading of a volume of vertebral trabecular bone (Figure 1.1). Applying data from previous studies [35,36,37], and assuming a linear relationship between apparent density and porosity allows apparent stiffness (elastic modulus, $E$ ) to be determined,

$E=E_{0} \times(1-p)^{b}$

in units of $\mathrm{MPa}$, where $p$ is porosity, and 14927 and 1.33 are values of $E_{0}$ and $\mathrm{b}$, respectively, for trabecular bone [18]. Bone mineral density (BMD) was calculated by a porosity relationship,

$B M D=\rho \times(1-p)$

where $\rho$ is apparent density of bone $\left(2.0 \mathrm{~g} / \mathrm{cm}^{3}\right.$ when $\left.p=0\right)$ [18]. Apparent density is the measure of mass per unit volume, including the voids spaces within the material.

Peak strain was calculated using Hooke's Law,

$\varepsilon=\sigma / E$.

The mechanical stimulus modeled to stimulate the bone volume's response to remodeling was defined as

$\Phi=R_{L} \times \varepsilon^{q}$

where $R_{L}$ is the loading frequency in cycles per day and $q$ adjusts the peak strain and loading frequency to correctly model the loading potential [18]. 


\subsection{Porosity Transformation}

The rate of change of porosity $(\dot{p})$ is the difference between the amount of bone formation and amount of bone resorption per given time period $[38,39]$. It is defined as $\dot{p}=Q_{R} N_{R}-Q_{F} N_{F}$,

where $Q_{R}$ and $Q_{F}$ are the mean bone resorbing and refilling (forming) rates, and $N_{R}$ and $N_{F}$ are the densities of resorbing and refilling BMUs per area, respectively. The rate of resorption,

$Q_{R}=A_{R} / T_{R}$,

and rate of refilling,

$Q_{\mathrm{F}}=A_{\mathrm{F}} / T_{\mathrm{F}}$,

are assumed to be linear in time [40]. The area of bone resorbed $\left(A_{R}\right)$ and area of bone formed $\left(A_{F}\right)$ were based on a cement line radius of $0.095 \mathrm{~mm}[40]$. When bone is in a disuse state $\left(\Phi<\Phi_{0}\right)$, refilling is reduced on bone surfaces [41]; thus, area of bone formed during disuse was reduced to $A\left[0.5+0.5\left(\Phi / \Phi_{0}\right)\right][18]$. The resorption $\left(T_{R}\right)$ and refilling $\left(T_{F}\right)$ periods were 25 and 64 days, respectively [23]. These periods were used to.

calculate total number of resorbing BMUs and refilling BMUs per given area. Integrating $\mathrm{BMU}$ activation frequency, $f_{\mathrm{a}}$ (BMUs/area/time), over a known time period will result in the number per section area of resorbing BMUs,

$$
N_{R}=\int_{i-T_{R}}^{t} f_{a}\left(t^{\prime}\right) d t^{\prime},
$$

and the number per section area of refilling BMUs,

$$
N_{F}=\int_{t-\left(T_{R}+T_{l}+T_{F}\right)}^{t-\left(T_{R}+T_{t}\right)} f_{a}\left(t^{\prime}\right) d t^{\prime} .
$$

Present time is $t$ and $T_{I}$ is a latency period between resorption and refilling [23]. 


\subsection{BMU Activation}

BMU activation frequency, as previously mentioned, is the number of active BMUs in the section area per day. It is assumed to be a function of the two remodeling mechanisms modeled by Hazelwood et al. [18], damage and disuse. Activation frequency, $f_{a}=\left(f_{a(\text { disuse })}+f_{a(\text { damage })}\right) S_{A}$,

is also a function of internal surface area as BMUs must begin on a bone surface [18]. $S_{A}$ is internal surface area per unit volume normalized to values between 0 and 1 by $S_{A \max }$. It was determined by using a porosity-surface area relationship developed by Martin [42] $S_{A}=\left(32.1 p-93.9 p^{2}+134 p^{3}-101 p^{4}+28.8 p^{5}\right) / S_{A \max }$

This accounts for decreased remodeling in bone volumes with smaller surface areas.

Changes in activation frequency due to damage and disuse were modeled assuming sigmoidal relationships [18] between damage and $f_{a(d a m a g e)}$

$$
f_{a(\text { damage })}=\frac{\left(f_{a 0}\right)\left(f_{a(\max )}\right)}{f_{a 0}+\left(f_{a(\max )}-f_{a 0}\right) e^{\left[k_{r}\left(f_{a(\max )}\right)\left(D-D_{0}\right) / \overline{D_{0}}\right]}},
$$

and mechanical stimulus and $f_{a(d i s u s e) \text {, }}$

$$
f_{a(\text { disuse })}=\frac{f_{a(\max )}}{1+e^{k_{b}\left(\Phi-k_{c}\right)}} \text { for } \Phi<\Phi_{0}
$$

The $k$ values were determined by matching the curves to clinical data [18]. Coefficients $k_{\mathrm{r}}$, $k_{\mathrm{b}}$, and $k_{\mathrm{c}}$ define the shape, slope, and inflection point of the curves, respectively. The maximum activation frequency $\left(f_{a(\max )}\right)$ of $0.50 \mathrm{BMUs} / \mathrm{mm}^{2} /$ day was higher than the highest measured activation frequency $\left(0.14 \mathrm{BMUs} / \mathrm{mm}^{2} /\right.$ day $)$ for human cortical bone because it is assumed the measurements did not reach the upper limits $[43,18]$. 


\subsection{Microdamage Accumulation}

Microdamage $(D)$ is defined as total crack length per section area of bone.

Damage accumulation due to fatigue is modeled according to Martin's work [44] as

$\dot{D}=\dot{D}_{F}-\dot{D}_{R}$

where $\dot{D}_{F}$ and $\dot{D}_{R}$ are the damage formation and removal rates, respectively. Based on Martin's findings, we assumed the rate of damage formation to be proportional to the loading potential [18],

$\dot{D}_{F}=k_{D} \times \Phi$

This model assumes a random distribution of BMUs and damage in the section area of bone; however, a damage removal specificity factor is included when modeling the rate of removal due to evidence showing that damage initiates the activation of BMUs $[9,10$, $14,15,18]$

$\dot{D}_{\mathrm{R}}=D f_{\mathrm{a}} A_{R} F_{s}$

The specificity factor, $F_{s}$, was assumed to be 5 based on experimental data [44]. Initial equilibrium is defined here as the time at which the rate of damage formation is equal to the rate of damage removal [18]. Setting both equations equal allows us to determine the damage rate coefficient,

$k_{D}=D_{0} f_{a 0} A F_{s} / \Phi$,

where initial, equilibrium values are designated by the subscript 0 . These values were obtained from Hazelwood et al. [18].

\subsection{Preflight Conditions}

Before applying microgravitational conditions, preflight parameters were calculated (Table 2.1) using constants derived from experimental data (Table 2.2). These 
parameter values were obtained by executing the model developed by Hazelwood et al. [18] until the values reached equilibrium. The applied stress on earth modeled to reach these values was determined by matching the predicted bone porosity of the model to 0.78, a porosity typical of vertebral trabecular bone in adult males in Earth's gravitational environment $[45,46,23]$. The calculated applied stress, $1 \mathrm{MPa}$, was based on a $100 \mathrm{~mm}^{2}$ cross-section from the modeled $1 \mathrm{~cm}^{3}$ trabecular bone volume (Figure 2.1) [23]. The resulting preflight BMD was $0.44 \mathrm{~g} / \mathrm{cm}^{3}$.

Table 2.1. Model parameters with values obtained from simulating remodeling in trabecular bone before entering space and at the end of a $\mathbf{1 8 0}$-day space simulation.

\begin{tabular}{|c|c|c|c|}
\hline & Description & $\begin{array}{c}\text { Preflight } \\
\text { trabecular bone }\end{array}$ & $\begin{array}{c}\text { End of flight values } \\
\text { (180-day) }\end{array}$ \\
\hline$E(\mathrm{MPa})$ & Elastic modulus & 1992.3 & 1881.9 \\
\hline$p$ & Porosity & 0.780 & 0.789 \\
\hline$B M D\left(\mathrm{~g} / \mathrm{cm}^{3}\right)$ & Bone mineral density & 0.4400 & 0.4215 \\
\hline$\varepsilon\left(10^{-6}\right)$ & Microstrain & 501.9 & 473.4 \\
\hline$\Phi\left(10^{-10}\right)$ & Loading potential & 1.904 & 1.507 \\
\hline $\mathrm{D}\left(\mathrm{mm} / \mathrm{mm}^{2}\right)$ & Total crack length per & 0.0375 & 0.0327 \\
\hline$\dot{D}_{F}\left(\mathrm{~mm} / \mathrm{mm}^{2} /\right.$ day $)$ & Damage formation area & 0.0000353 & 0.0000280 \\
\hline$\dot{D}_{R}\left(\mathrm{~mm} / \mathrm{mm}^{2} /\right.$ day $)$ & Damage removal rate & 0.0000353 & 0.0000417 \\
\hline$f_{a}\left(\mathrm{BMUs} / \mathrm{mm}^{2} /\right.$ day $)$ & $\begin{array}{c}\text { BMUs appearing in the } \\
\text { section area per day }\end{array}$ & 0.0133 & 0.0180 \\
\hline$N_{F}\left(\# / \mathrm{mm}^{2}\right)$ & Number of refilling sites & 0.8495 & 1.2825 \\
\hline$N_{R}\left(\# / \mathrm{mm}^{2}\right)$ & Number of resorbing sites & 0.3318 & 0.4483 \\
\hline
\end{tabular}


Table 2.2. Remodcling simulation constants.

\begin{tabular}{|c|c|c|c|}
\hline Constant (units) & Description & $\begin{array}{c}\text { Nominal } \\
\text { value }\end{array}$ & Source \\
\hline$A_{F}\left(\mathrm{~mm}^{2}\right)$ & Area of formation & $1.418 \times 10^{-2}$ & {$[23]$} \\
\hline$A_{R}\left(\mathrm{~mm}^{2}\right)$ & Area of resorption & $1.418 \times 10^{2}$ & {$[23]$} \\
\hline$A_{R(1.2)}\left(\mathrm{mm}^{2}\right)$ & Area of resorption (reduced by $1 / 6$ ) & $1.181 \times 10^{2}$ & {$[23]$} \\
\hline$A_{R(l .3)}\left(\mathrm{mm}^{2}\right)$ & Area of resorption (reduced by $3 / 13$ ) & $1.090 \times 10^{2}$ & {$[23]$} \\
\hline$T_{R}$ (days) & Resorption period & 25 & {$[23]$} \\
\hline$T_{I}$ (days) & Reversal period & 5 & {$[23]$} \\
\hline$T_{F}$ (days) & Refilling period & 64 & [23] \\
\hline$k_{D}\left(\mathrm{~mm} / \mathrm{mm}^{2}\right)$ & Damage rate coefficient & $\sim 1.85 \times 10^{5}$ & {$[18]$} \\
\hline$R_{L}(\mathrm{cpd})$ & Loading rate & 3000 & {$[18]$} \\
\hline$q$ & Damage rate exponent & 4 & {$[47]$} \\
\hline$F_{s}$ & Damage removal specificity factor & 5 & {$[44]$} \\
\hline$D_{0}\left(\mathrm{~mm} / \mathrm{mm}^{2}\right)$ & Initial damage & 0.0366294 & {$[14,48]$} \\
\hline$f_{a 0}\left(\mathrm{BMUs} / \mathrm{mm}^{2} /\right.$ day $)$ & Initial BMU activation frequency & 0.0067 & {$[49]$} \\
\hline$\Phi_{0}(\mathrm{cpd})$ & Initial mechanical stimulus & $1.875 \times 10^{-10}$ & {$[18]$} \\
\hline$\sigma_{e}(\mathrm{MPa})$ & Stress applied on earth & 1 & \\
\hline$\sigma_{s}(\mathrm{MPa})$ & Stress applied in space & 0.8909 & \\
\hline $\begin{array}{c}f_{a(\max )} \\
\left(\mathrm{BMUs} / \mathrm{mm}^{2} / \mathrm{day}\right)\end{array}$ & Maximum BMU activation frequency & 0.5 & {$[18]$} \\
\hline$S_{A(\max )}$ & Max. specific surface area, normalizing constant & 4.195 & {$[18]$} \\
\hline$k_{b}\left(\mathrm{cpd}^{-1}\right)$ & Activation frequency dose-response coefficient & $6.5 \times 10^{10}$ & {$[18]$} \\
\hline$k_{c}(\mathrm{cpd})$ & Activation frequency dose-response coefficient & $9.4 \times 10^{-1 t}$ & {$[18]$} \\
\hline$k_{r}$ & Activation frequency dose-response coefficient & -1.6 & {$[18]$} \\
\hline
\end{tabular}

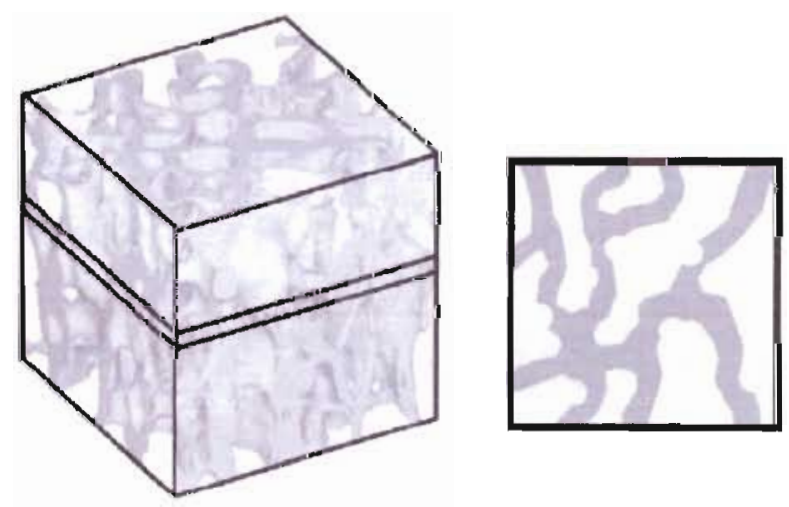

Figure 2.1. Trabecular volume and cross-section [23]. 


\subsection{Simulation of Spaceflight}

Microgravity was simulated by lowering the stress applied to the bone volume. Astronauts experienced an average vertebral trabecular BMD loss of $0.7 \%$ per month, for a total of $4.2 \%$ BMD loss during the 180 -day average ISS missions $[3,5,6]$. For this simulation, stress applied to the representative volume during spaceflight was then determined as $0.8909 \mathrm{MPa}$ based on this 180-day spaceflight ending density of 0.4215 $\mathrm{g} / \mathrm{cm}^{3}$

Using the density loss values at 180 days as a baseline, various durations of spaceflight were simulated based on typical mission length for astronauts, including 10 days, 90 days, and 180 days $[5,4]$. Though 365 days in space is not typical, it was also simulated to examine the potential of bisphosphonates to maintain BMD without increasing damage.

\subsection{Simulation of Bisphosphonates}

Bisphosphonate treatment was simulated using two factors: one lowering activation frequency and the other reducing the resorption area. A potency variable $(P)$, where $0 \leq P \leq 1$, is applied to exert the former effect by multiplying $f_{a}$ by the quantity ( 1 $-P) . P$ is based on pharmacokinetic properties of bisphosphonates, including potency factors, binding, uptake, and mode of action [23],

$P=P_{\max }\left(1-\mathrm{e}^{-\tau \mathrm{s}_{\mathrm{x}} N_{\mathrm{R}}}\right)$.

$P_{\max }$ and $T_{\mathrm{s}}$ are suppression coefficients reflecting various properties of bisphosphonates in order to model a range of drug potencies. Values for these coefficients (Table 2.3) were selected based on the experimental results from 1 year studies of daily alendronate and daily pamidronate treatment, and modeled the variations in the reduction of 
activation frequency as seen in these studies [23]. Figure 2.2 displays the effects of the coefficients on the relationship between $P$ and $N_{R}$.

Table 2.3. Bisphosphonate effects analyzed during the simulation.

\begin{tabular}{|c|c|c|c|c|c|}
\hline Treatment & Label & $\begin{array}{c}\text { Level of } \\
\text { suppression }\end{array}$ & $\boldsymbol{P}_{\max }$ & $T_{\mathrm{s}}$ & $\begin{array}{c}\text { Initial } \\
\text { bone } \\
\text { balance }\end{array}$ \\
\hline no treatment & P0a1 & - & 0 & 0 & 1 \\
\hline$A$ & P07t5a12 & Low & 0.7 & 5 & 1.2 \\
\hline$B$ & P07t20a12 & \multirow{2}{*}{ Intermediate } & 0.7 & 20 & 1.2 \\
\hline$C$ & $\mathrm{P} 1 \mathrm{t5a} 12$ & & 1.0 & 5 & 1.2 \\
\hline$D$ & P1t20a12 & High & 1.0 & 20 & 1.2 \\
\hline$E$ & P07t5a13 & Low & 0.7 & 5 & 1.3 \\
\hline$F$ & P07t20a13 & \multirow{2}{*}{ Intermediate } & 0.7 & 20 & 1.3 \\
\hline$G$ & P1t5a13 & & 1.0 & 5 & 1.3 \\
\hline$H$ & Płt20a13 & High & 1.0 & 20 & 1.3 \\
\hline
\end{tabular}

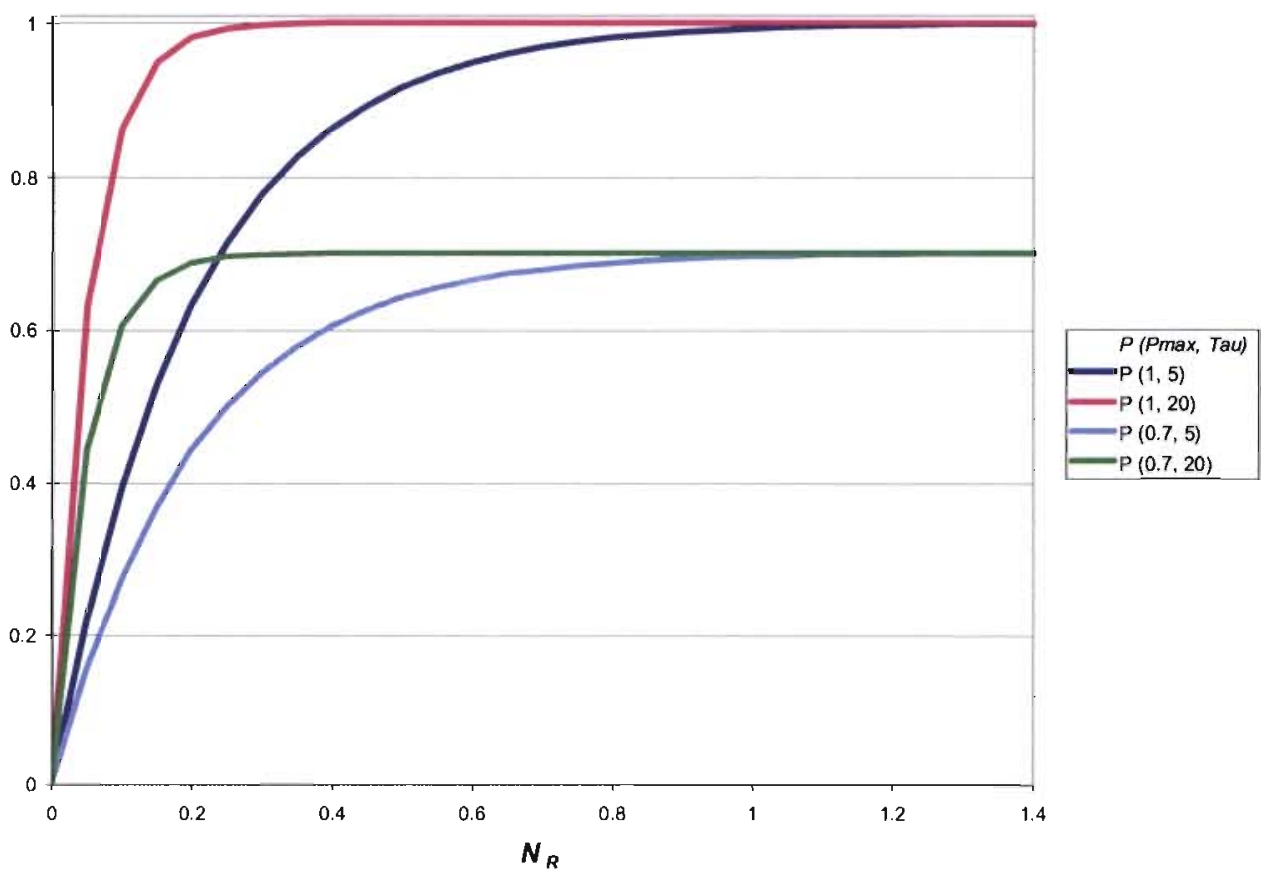

Figure 2.2. Bisphosphonate potency as a function of number of resorbing BMUs.

The size of the resorption cavity is reduced during bisphosphonate treatment due to their effects on osteoclasts [23], resulting in alterations to the ratio of bone area formed to bone area removed. Two different initial bone balance ratios $\left(A_{F} / A_{R}\right)$ for the simulation 
of bisphosphonate treatment were used (Table 2) based on reductions of $1 / 6$ and 3/13 to resorption area found in postmenopausal women treated with bisphosphonates for 1 year [23]. A bone balance of 1.0 is assumed for the simulation when bisphosphonate treatment is not in effect.

Bisphosphonates were simulated during the entire spaceflight. Preflight treatment was also examined, where bisphosphonates were applied to the simulation at $0,7,14,30$, 90 , and 180 days preflight.

\subsection{Simulation of Return to Earth}

The return to earth was modeled by resuming preflight bone loading conditions, with an applied stress of $1 \mathrm{MPa}$. Once back on Earth, bisphosphonate therapy was discontinued. The simulation was extended 365 days postflight to examine increases or decreases in fracture risk based on bone mineral density and damage accumulation. Though it takes 1 to 3 years to fully recover without treatment, a one year postflight examination allowed us to determine if a treatment is successful at maintaining bone mass without accumulating more damage. A successful treatment will cause the remodeling properties to reach new equilibrium values within one complete remodeling cycle ( 3 to 4 months) after arriving back on Earth.

\subsection{Model Implementation}

The computational model was coded in MATLAB (Appendix A). The time increment for which all the model variables were updated and tracked was 1 day. The computational simulation was executed in Windows Vista (32-bit) using an Intel Core 2 Duo processor. 


\subsection{Postflight Parameter Analysis}

Together, bone mass (quantity) and bone quality determine the ability of bone to resist fracture [50,51]. For this simulation, we examined changes in bone mass and bone quality in terms of BMD and microdamage accumulation, respectively. Experimental results from ex vivo studies have shown BMD to predict 66 to 74 percent of the variation in bone strength [52]. An increase in bone mass and bone quality will lead to stronger, more fracture resistant bone; thus, in the analysis we assumed higher BMD and lower damage accumulation to lead to higher fracture resistance. 


\section{CHAPTER 3: RESULTS}

\subsection{Untreated Spaceflight}

The model's results were consistent with experimental data obtained from spaceflight [4] in that the predicted BMD loss was non-linear (Figure 3.1). Predicted damage accumulation decreased as flight duration increased (Figure 3.1) due to the disuse response in which damage was specifically targeted during resorption and because less damage formed each day due to the microgravity environment (Figure D25). The predicted rate of BMD loss was greatest early on in the flight, showing smaller decrements as time spent in space increased. The model predicted little BMD loss and change from preflight damage for the typical flight duration of 10 days (Figure 3.1); however, postflight predictions showed that BMD continued to decrease upon return to Earth until it reached a value nearly 3 times lower than it was at the end of the mission before increasing to near normal levels about 100 days after entering Earth's gravity (Figure B1). 


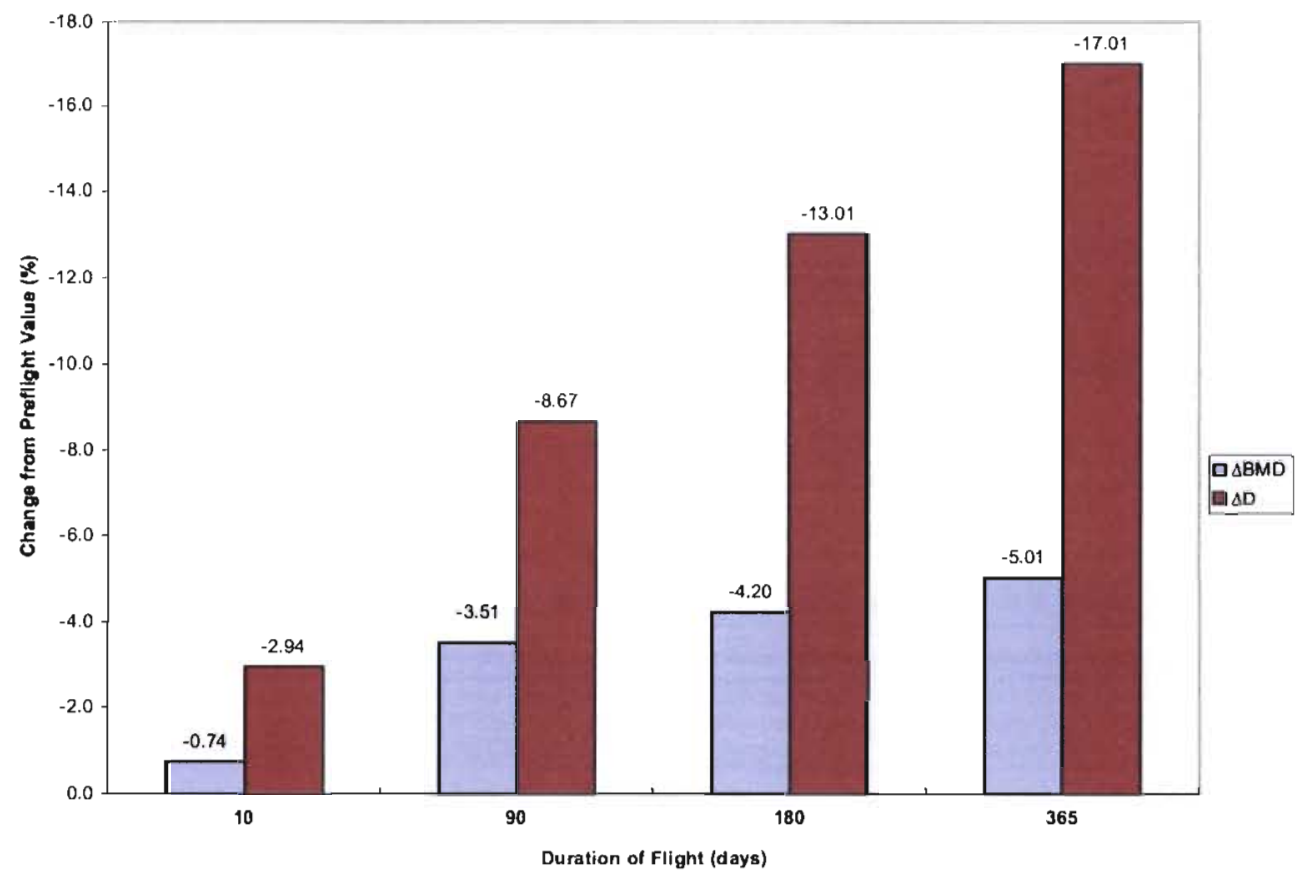

Figure 3.1. Predicted percent decreases in BMD and damage (D) of untreated bone at end of spaceflight.

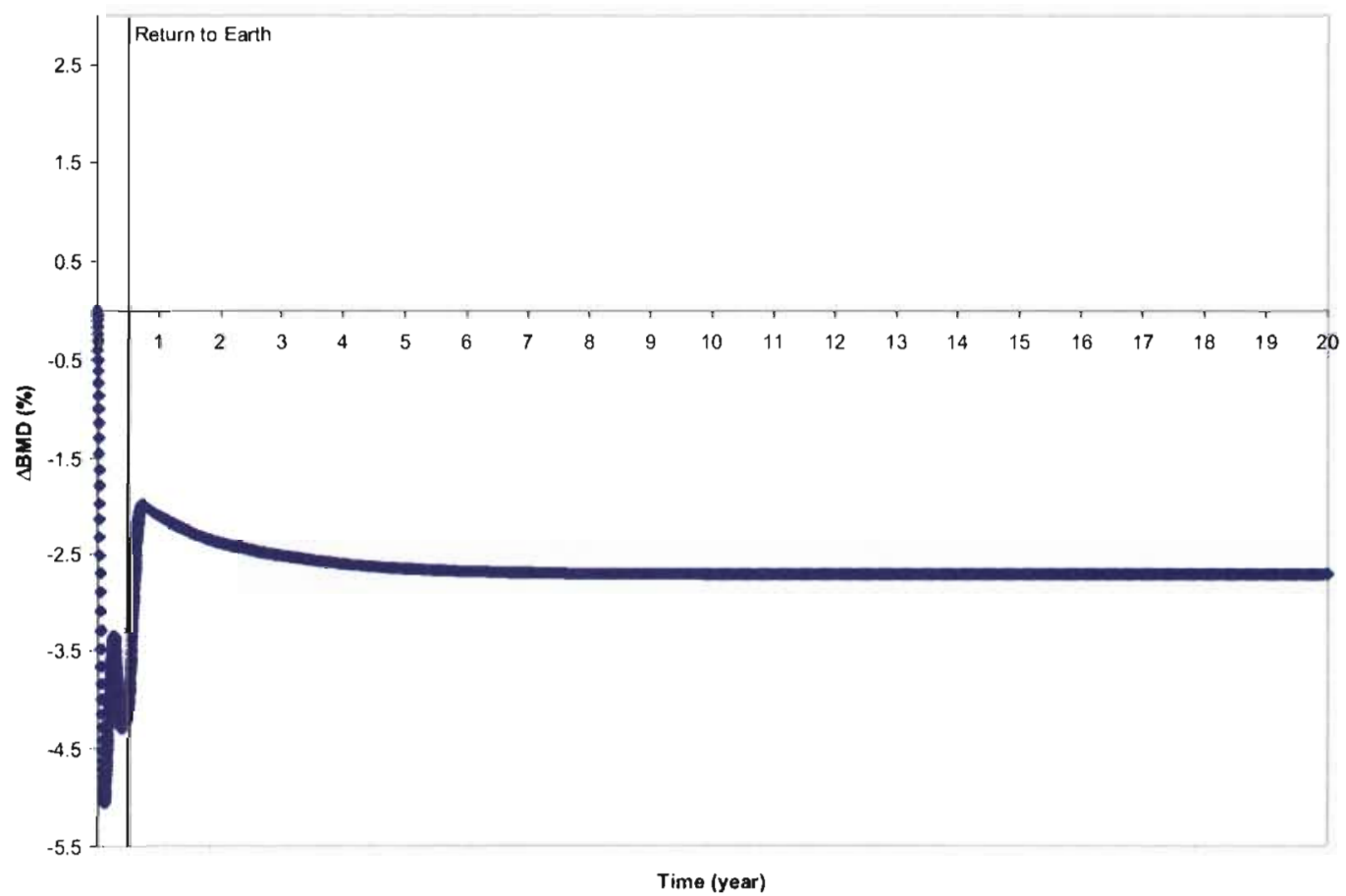

Figure 3.2. Predicted effects of 180-day spaceflight on BMD of untreated bone from beginning of flight through 19.5 years postflight. 


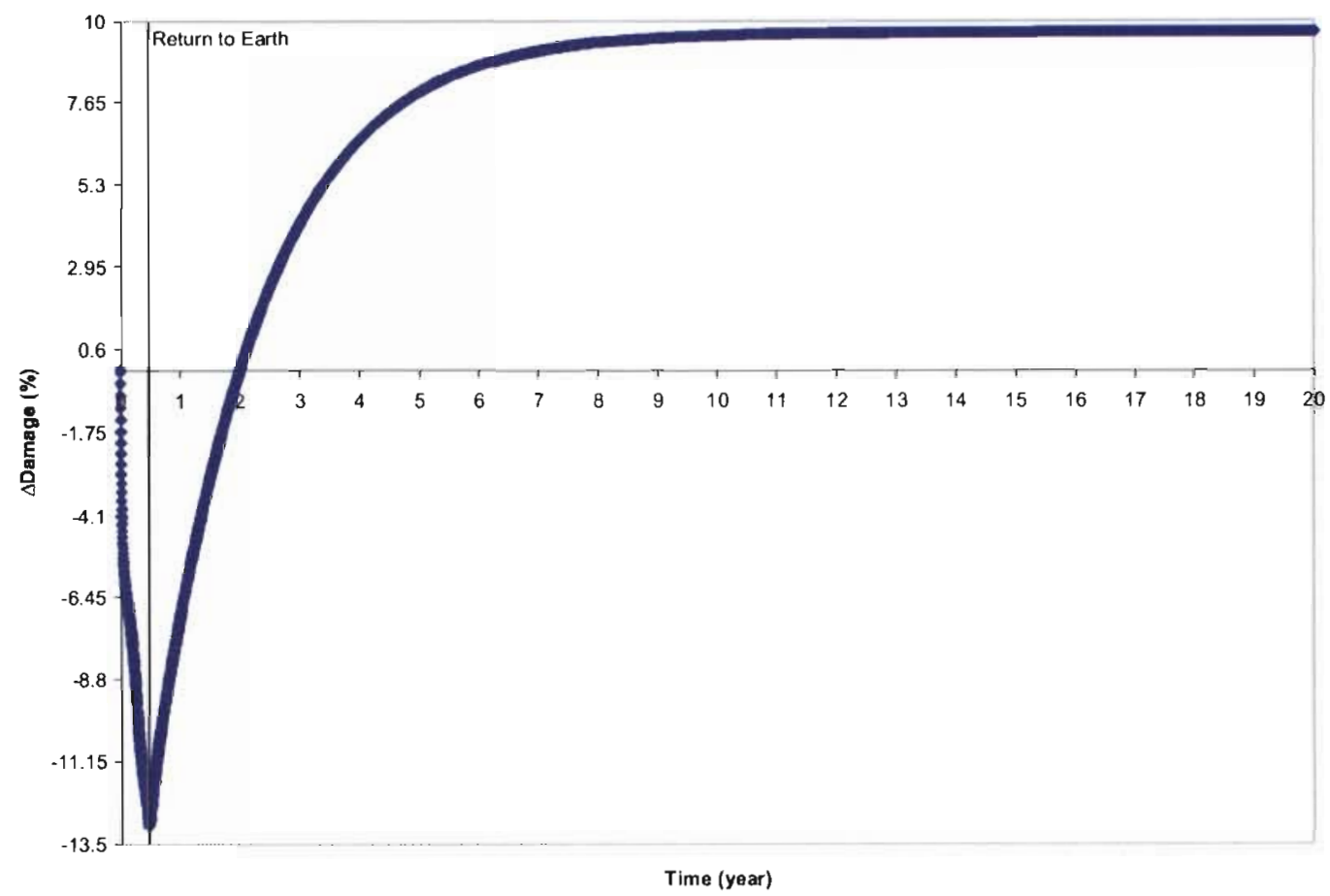

Figure 3.3. Predicted effects of 180-day spaceflight on damage accumulation (D) of untreated bone from beginning of flight through 19.5 years postflight.

For the 180-day space mission, the predicted postflight results for untreated bone showed sharp increases to both BMD and damage upon return to Earth (Figures 3.2 and 3.3). BMD reached equilibrium approximately 7 years postflight, and was about $2.7 \%$ less than its preflight value. Damage continued to accumulate postflight until equilibrium was reached about 15 years after the return to Earth. Though damage was initially lower upon return to earth, it continued to increase postflight until reaching a value approximately $9.5 \%$ higher than its preflight value. Figures 3.2 and 3.3 clearly indicate a need for treatment as bone was negatively affected by decreased mass and quality.

Also noteworthy is the predicted oscillatory behavior of BMD values seen in disuse (Figures 3.2 and 3.15) for untreated bone. The inflection points are located approximately at the same intervals as the transitions from resorption to refilling and 
refilling back to resorption in the BMU remodeling period. This oscillatory trend was also noted in the model by Hazelwood et al. [18] and still needs further investigation.

\subsection{Bisphosphonate Treatment Onset at Beginning of Spaceflight}

The simulation predicted gains in BMD for shorter flights (10 and 90 days) in which treatments exhibited intermediate to high suppression (Figures 3.4 and 3.5). For all flight durations, high suppression of BMU activation (Table 2.3) led the model to predict increases in both BMD and damage accumulation. Overall, the model predicted treatments creating bone balances of 1.3 to have more positive effects on BMD (i.e. less loss or greater increase). Although those with bone balances of 1.3 positively affected $\mathrm{BMD}$, the model predicted that they also caused more damage to accumulate when the level of remodeling suppression was high. In general, the model predicted treatments with intermediate levels of suppression to have smaller changes in BMD at the end of spaceflight than the decreases predicted with low levels of suppression and than the increases predicted with high levels of suppression. Also, the simulation predicted that treatments with intermediate suppression caused end-of-flight BMD and damage accumulation values of to remain closer to the pretreatment, preflight values than treatments with low or high remodeling suppression.

As flight duration increased, predicted percent changes in damage and BMD increased. Simulations of long flight durations (180 and 365 days) resulted in significant changes, predicting treatments with low and intermediate suppression to have significant decreases in damage (up to $14.5 \%$ ) similar to untreated bone. The greatest predicted increases in both damage and BMD occurred for treatments of high suppression and bone

balances of 1.3. Simulation of treatments C, E, F, and G (P1t5a12, P07t5a13, P07t20a13, 
and P1t5a13), with low and intermediate levels of remodeling suppression, showed increases in BMD and decreases in damage accumulation on longer duration spaceflights (Figures 3.6 and 3.7).

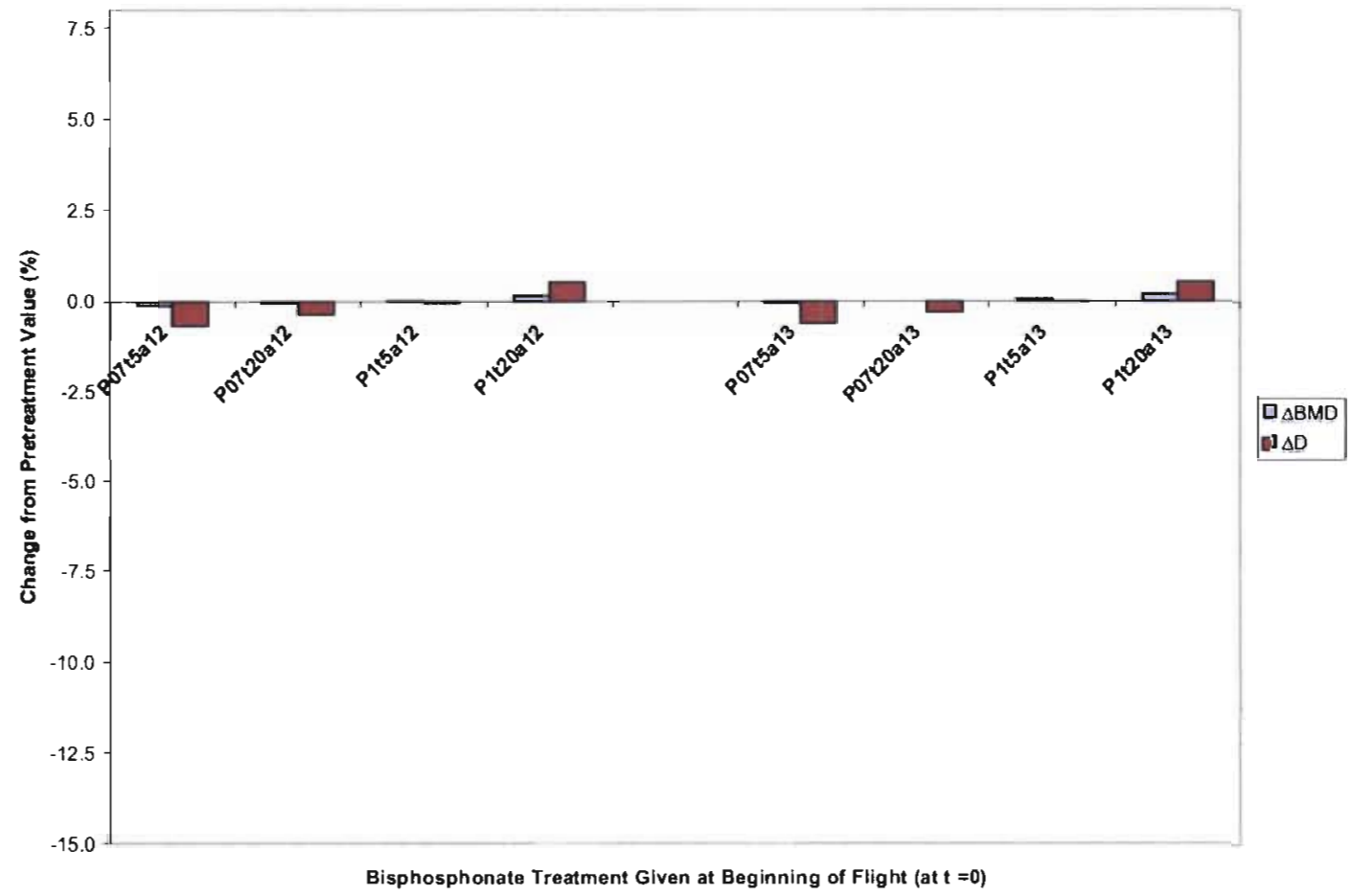

Figure 3.4. Predicted percent changes in BMD and damage (D) at end of 10-day spaceflight. 


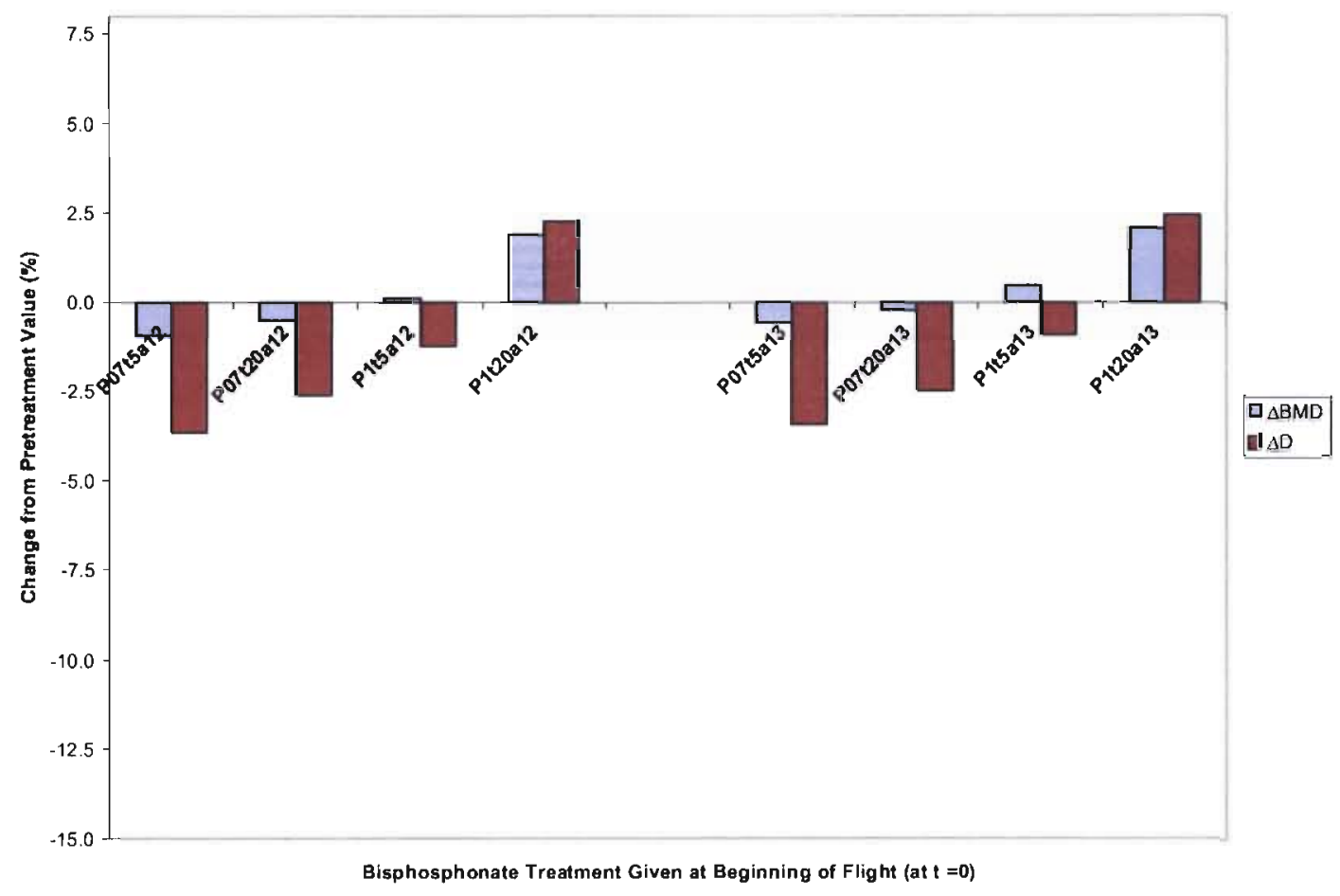

Figure 3.5. Predicted percent changes in BMD and damage (D) at end of 90-day spaceflight.

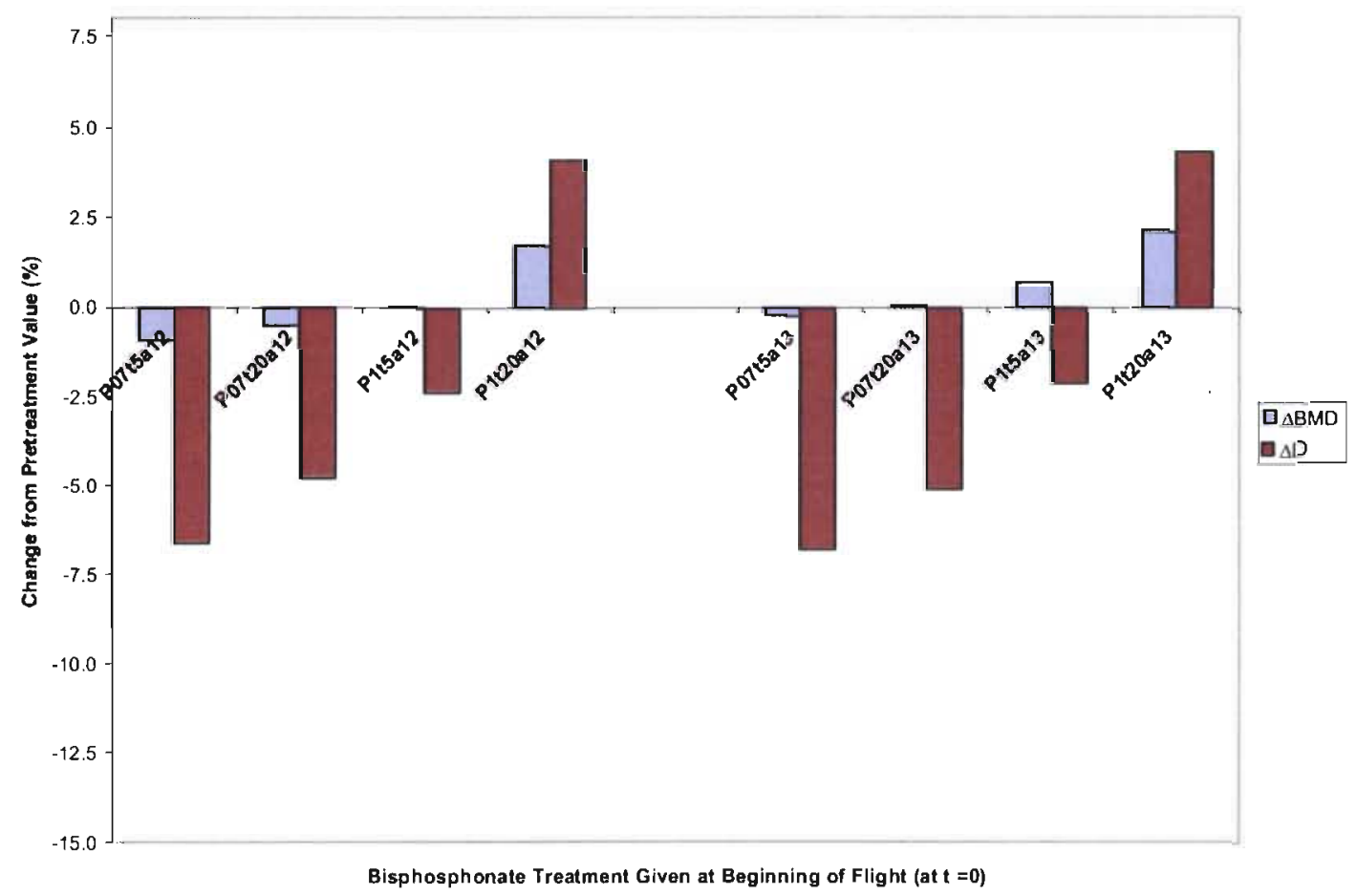

Figure 3.6. Predicted percent changes in BMD and damage (D) at end of 180-day spaceflight. 


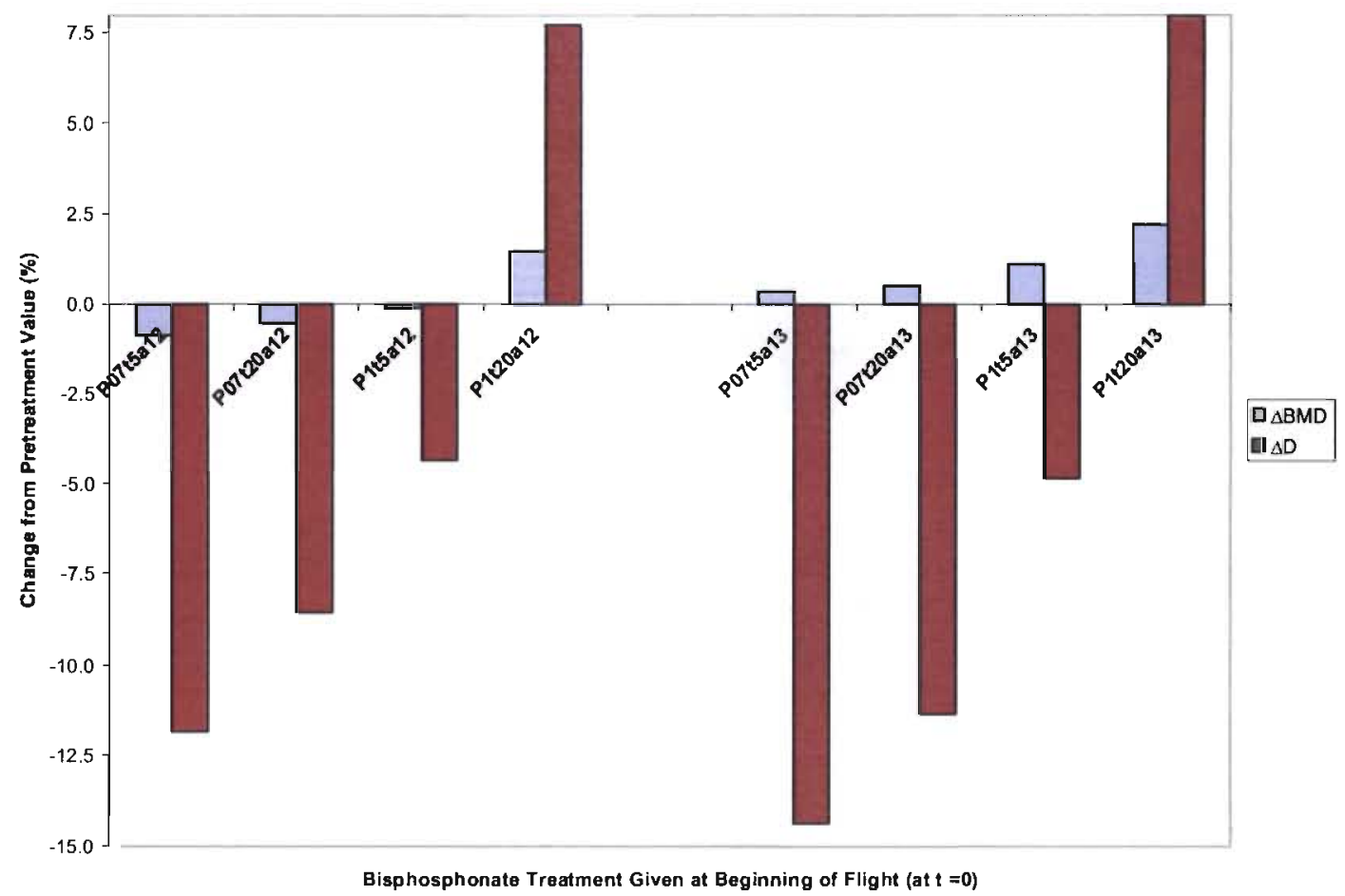

Figure 3.7. Predicted percent changes in BMD and damage (D) at end of 365-day spaceflight.

\subsection{Preflight Bisphosphonate Treatment}

In accordance with data obtained from clinical studies [21], the predicted results of bisphosphonate treatment on Earth exhibited increases in BMD and damage accumulation. Predicted pre-spaceflight additions to BMD and damage continued to rise as the duration of the preflight therapy simulation increased from 7 to 180 days (Figures 3.8-3.12). The model showed damage to increase at a faster rate than BMD. The ratio of percent increase in damage to percent increase in $\mathrm{BMD}$, used in this study as a measure indicating efficiency of reducing fracture risk in which a lower value is more optimal, was highest for treatments with high levels of remodeling suppression, and especially for those also with lower bone balances (Table 3.1). The lowest ratio of percent preflight increase of damage to BMD was predicted for Treatment $\mathrm{E}$ (P07t5a13), which modeled 
low remodeling suppression and a bone balance ratio of 1.3 . Bisphosphonate treatments with lower suppression of BMU activation and higher bone balance ratios proved to be optimal by adding more healthy bone per increase in BMD.

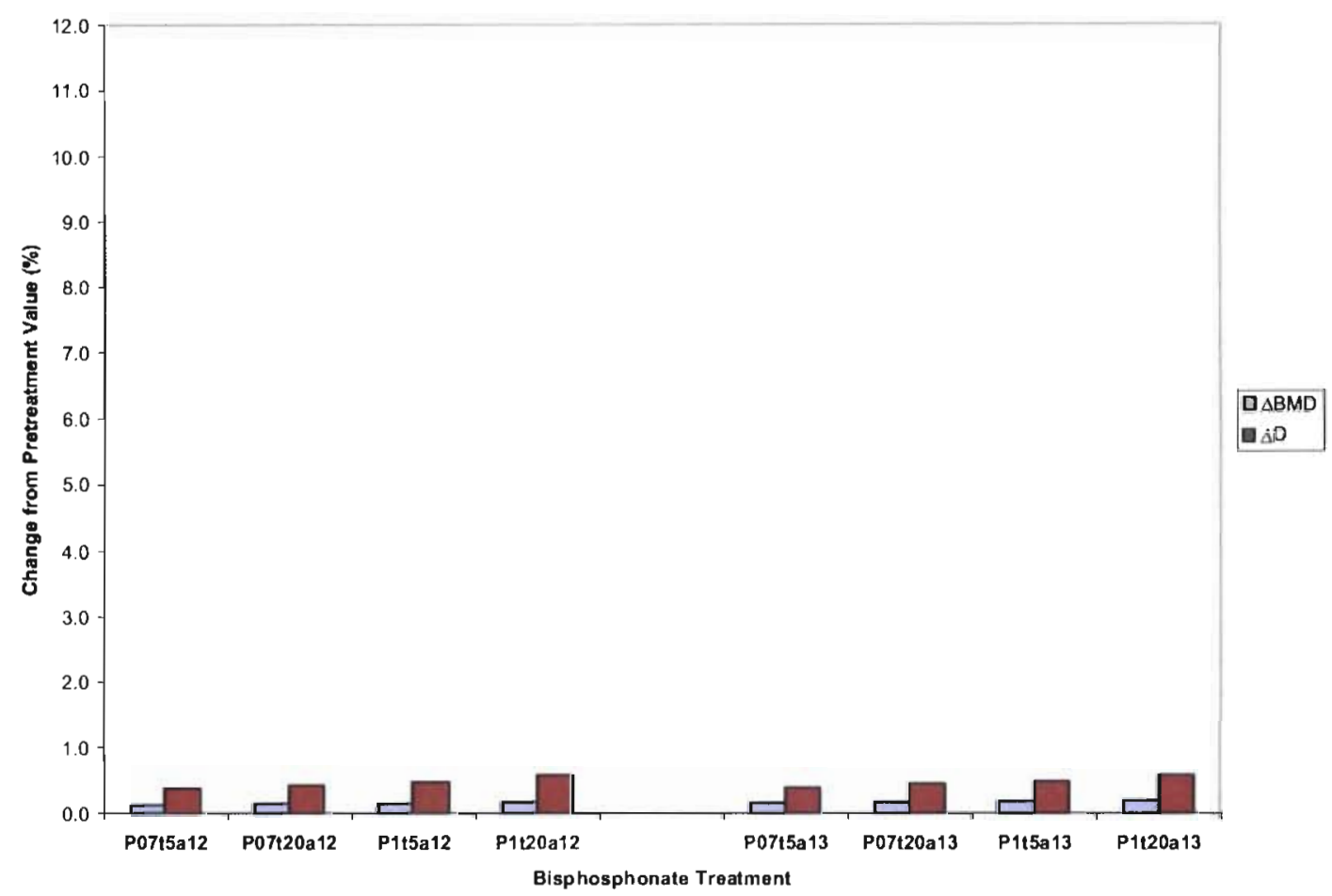

Figure 3.8. Predicted preflight increase in BMD and damage (D) due to 7-day preflight treatment. 


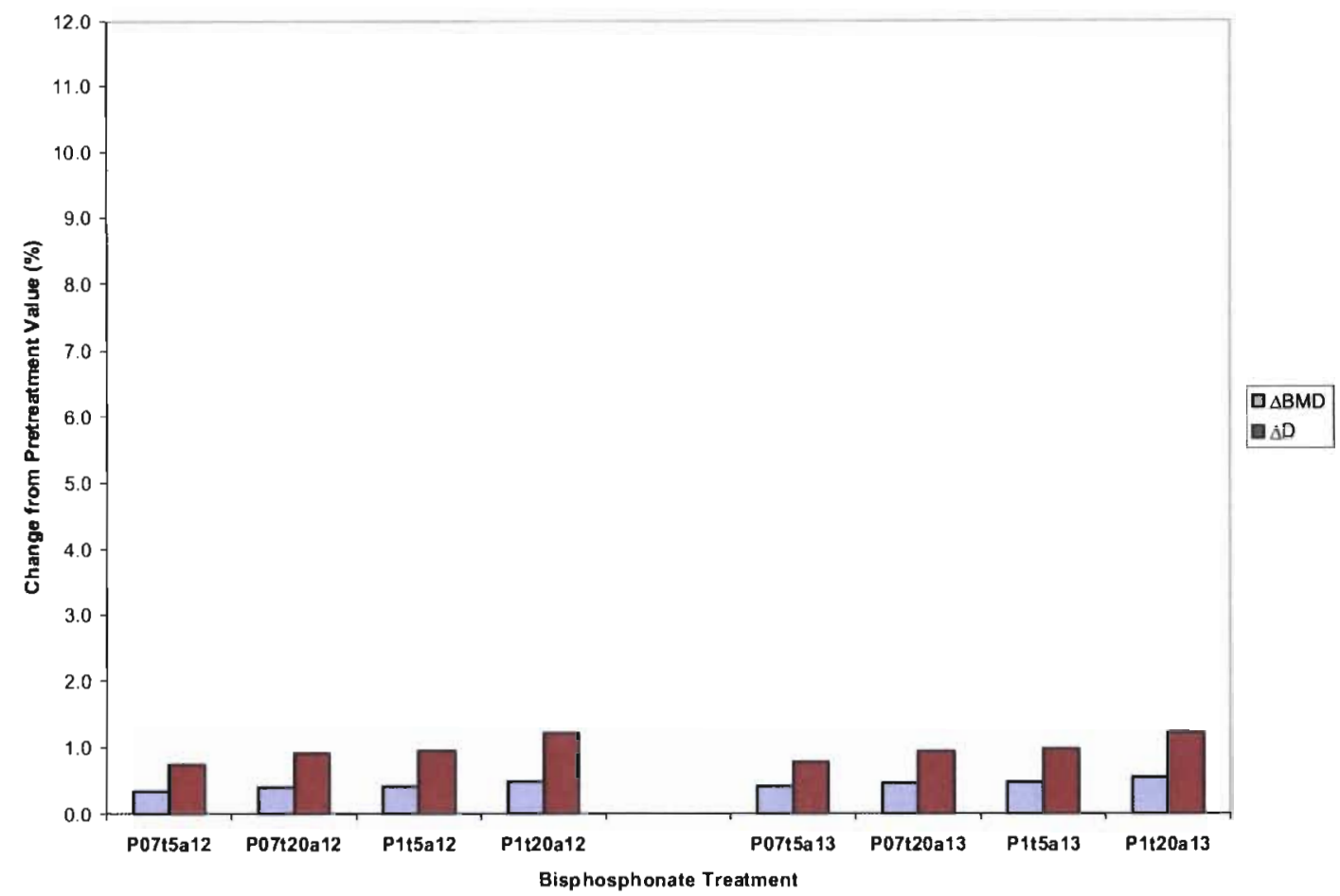

Figure 3.9. Predicted preflight increase in BMD and damage (D) due to 14-day preflight treatment.

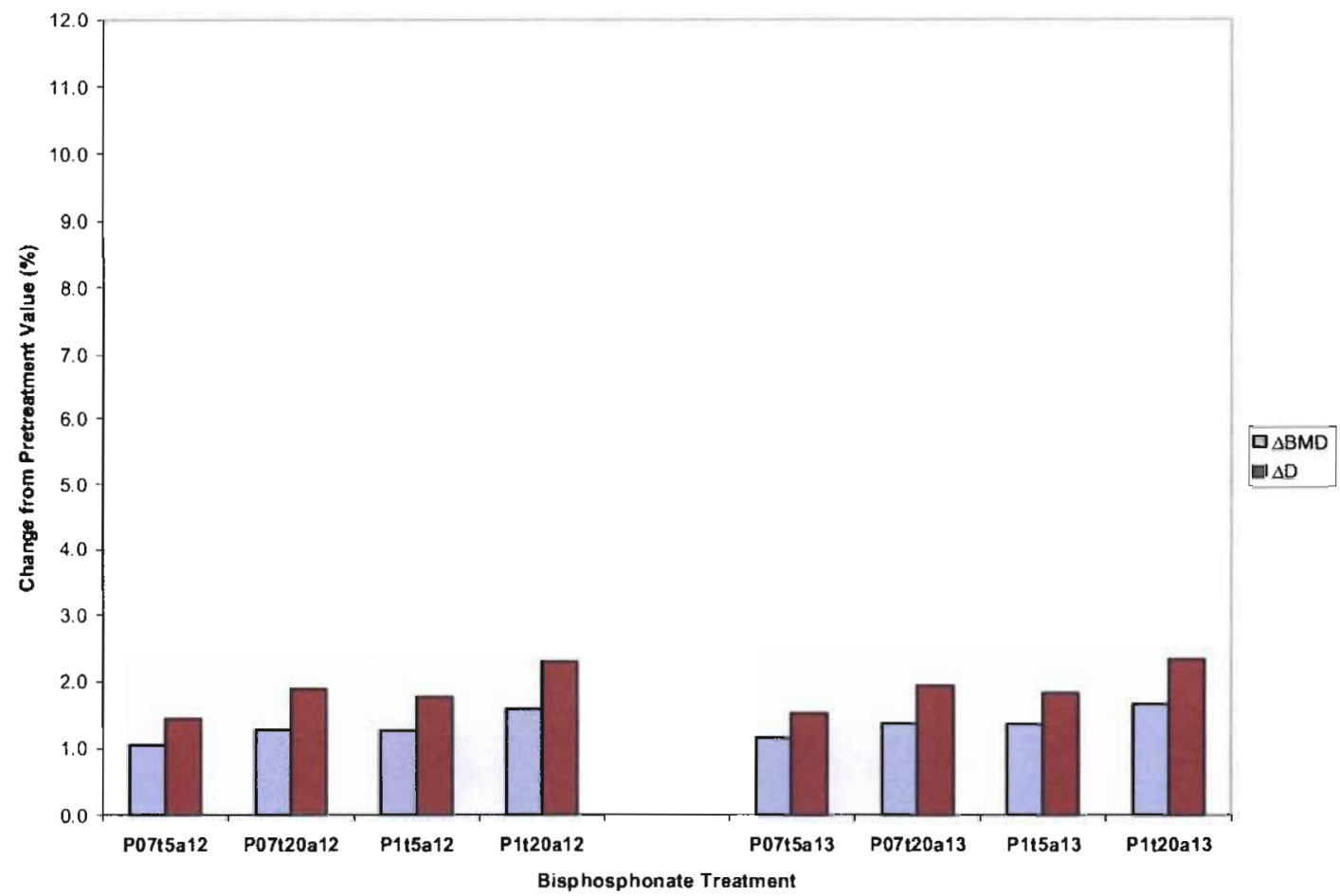

Figure 3.10. Predicted preflight increase in BMD and damage (D) due to 30-day preflight treatment. 


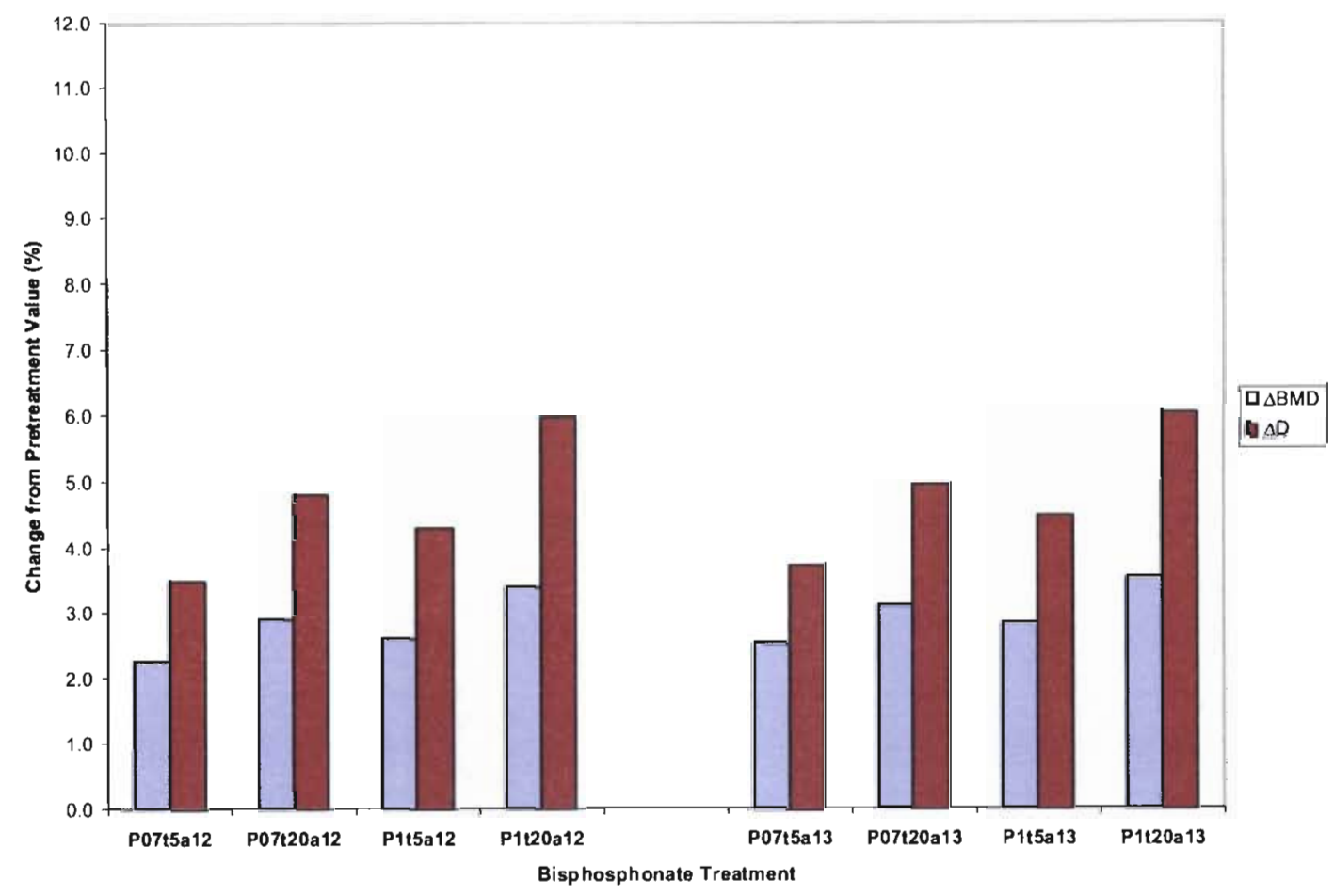

Figure 3.11. Predicted preflight increase in BMD and damage (D) due to 90-day preflight treatment.

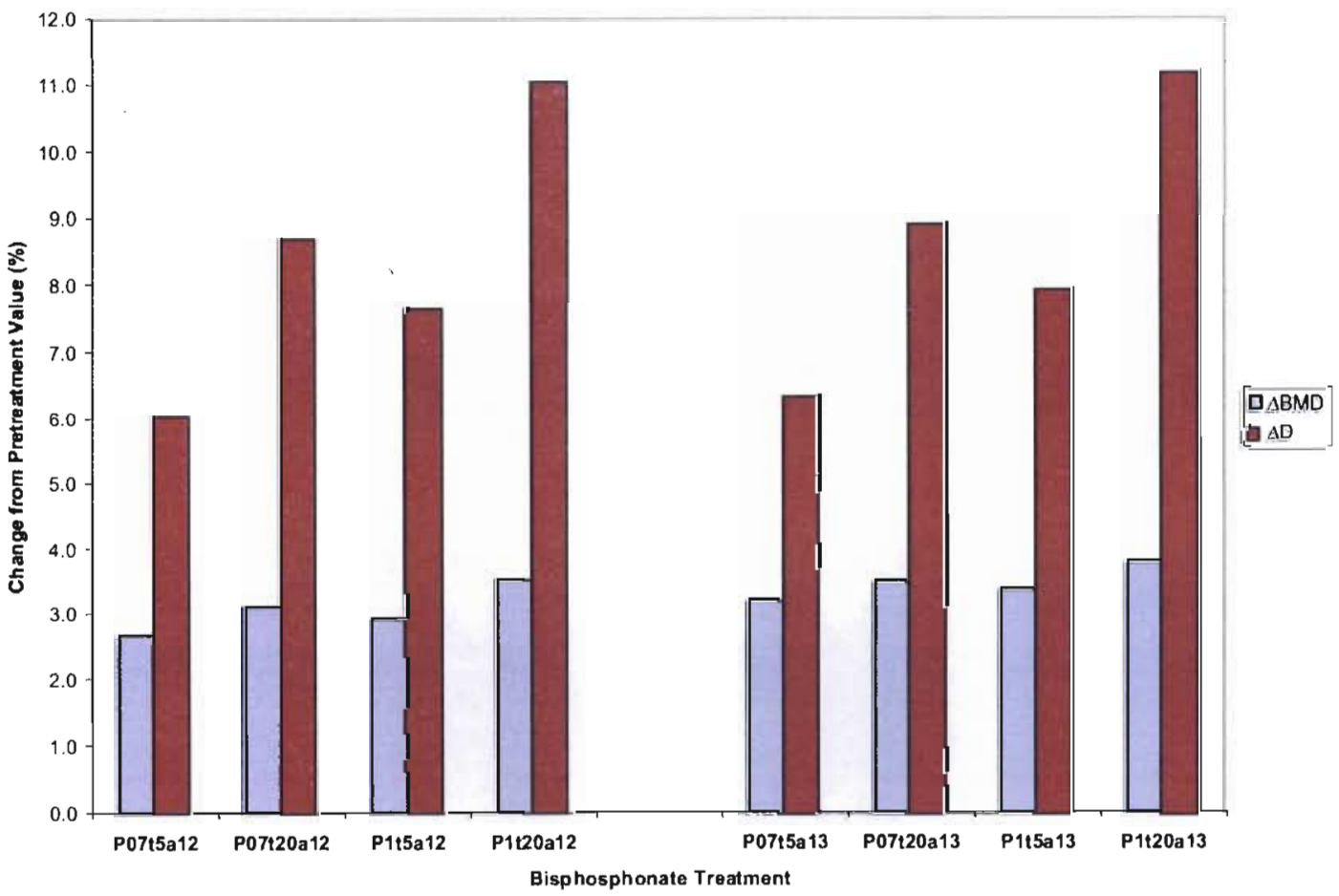

Figure 3.12. Predicted preflight increase in BMD and damage (D) due to 180-day preflight treatment. 
Table 3.1. Ratio of percent preflight increase of damage (D) to BMD.

\begin{tabular}{|c|c|c|c|c|c|}
\cline { 2 - 6 } \multicolumn{1}{c|}{} & \multicolumn{4}{c}{$\% \Delta \mathrm{D}: \% \Delta \mathrm{BMD}$ for Number of Days of Preflight Treatment } \\
\cline { 2 - 6 } & $\mathbf{7}$ & $\mathbf{1 4}$ & $\mathbf{3 0}$ & $\mathbf{9 0}$ & $\mathbf{1 8 0}$ \\
\hline P07t5a12 & 2.83 & 2.08 & 1.37 & 1.55 & 2.25 \\
\hline P07t20a12 & 2.91 & 2.30 & 1.48 & 1.66 & 2.80 \\
\hline P1t5a12 & 3.17 & 2.29 & 1.39 & 1.66 & 2.62 \\
\hline P1t20a12 & 3.39 & 2.49 & 1.44 & 1.76 & 3.14 \\
\hline P07t5a13 & 2.44 & 1.88 & 1.32 & 1.47 & 1.98 \\
\hline P07t20a13 & 2.52 & 2.04 & 1.42 & 1.60 & 2.55 \\
\hline P1t5a13 & 2.72 & 2.04 & 1.34 & 1.58 & 2.35 \\
\hline P1t20a13 & 2.92 & 2.26 & 1.40 & 1.71 & 2.96 \\
\hline
\end{tabular}

\subsection{Varying Onset of Bisphosphonate Treatment for Spaceflight}

For longer durations in space, the model predicted pretreatment periods to become less effective at influencing the end-of-flight values for BMD and damage (Figure 3.7, 3.13, and 3.14). As duration in space increased, the model predicted end-of-flight values to be influenced more by the effects of spaceflight than the effects of preflight treatment.

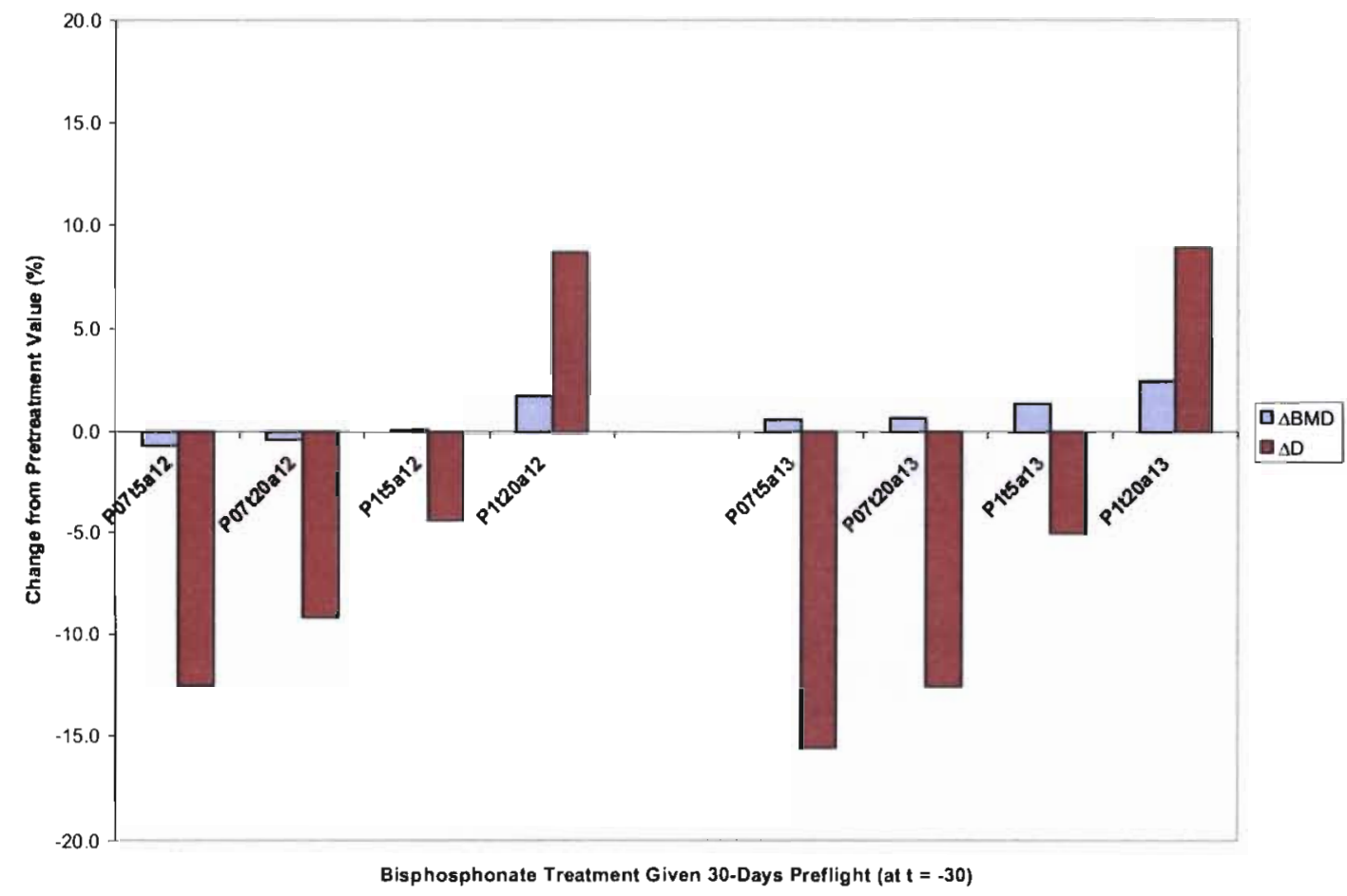

Figure 3.13. Predicted percent changes in BMD and damage (D) at end of 365-day spaceflight due to 30-day preflight treatment. 


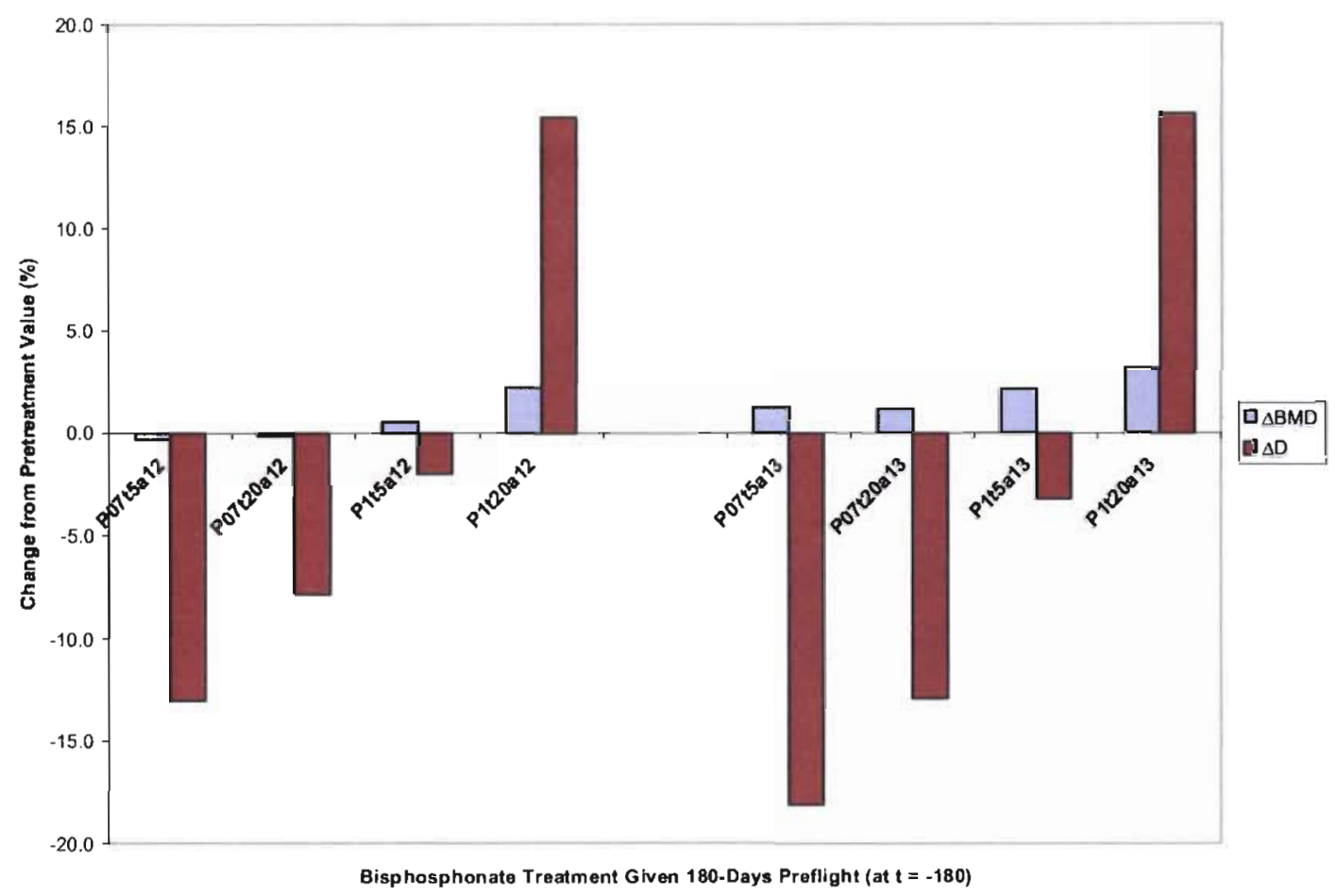

Figure 3.14. Predicted percent changes in BMD and damage (D) at end of 365-day spaceflight due to 180-day preflight treatment.

Alternately, pretreatment phases highly influenced the model's predictions of BMD and damage for shorter durations of spaceflight. For nearly all bisphosphonate potencies simulated, the addition of preflight treatment caused increased BMD and damage accumulation at the end of 10-day spaceflight. For 90-day spaceflight, a pretreatment period of 30 days predicted increases in BMD without significantly increasing damage, and the same occurred with a 90 -day pretreatment for 180-day spaceflight. Also, adding preflight treatment for therapies with high suppression predicted further increases to the already large gains in BMD and damage that occurred without pretreatment.

\subsection{One-Year Postflight, Posttreatment Recovery}

The model predicted treatments with low levels of remodeling suppression given initially at the beginning of spaceflight to result in the highest BMD and lowest 
microcrack density accumulation 1-year after returning to Earth (which is also 1-year posttreatment). Treatments with high suppression, though they resulted in higher BMD at the end of flight, were predicted to generate the lowest BMD of all the applied treatments. Predicted 1-year postflight values for BMD and damage for treatments with intermediate levels of remodeling suppression were between the two extremes (Figures 3.15 and 3.16). Note that bisphosphonate treatment begins where the $\mathrm{x}$ - and $\mathrm{y}$-axes meet for the line graphs that display timelines of preflight through 1-year postflight predicted values.

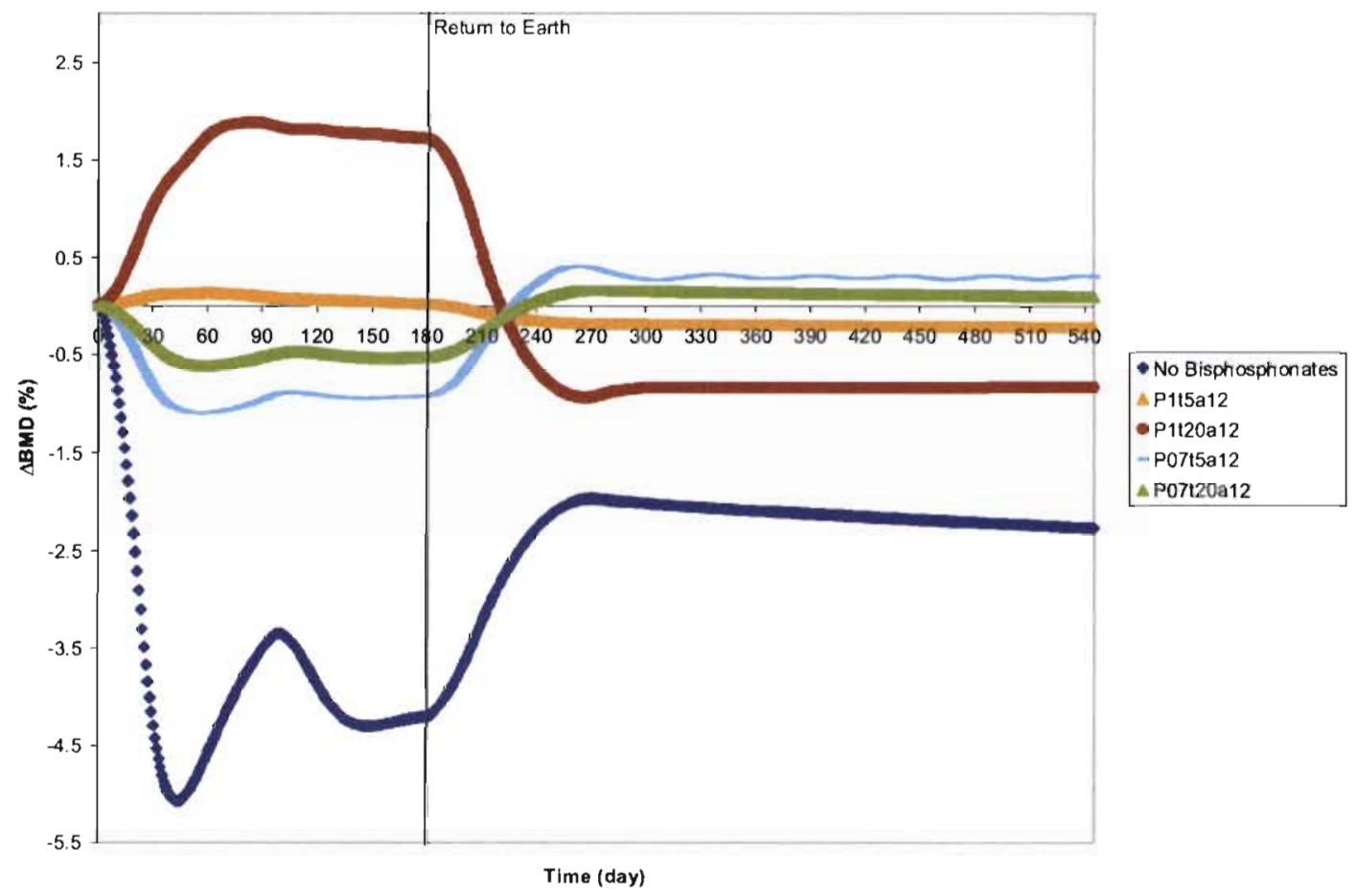

Figure 3.15. Predicted bisphosphonate effects on BMD and posttreatment return to Earth from 180day spaceflight. 


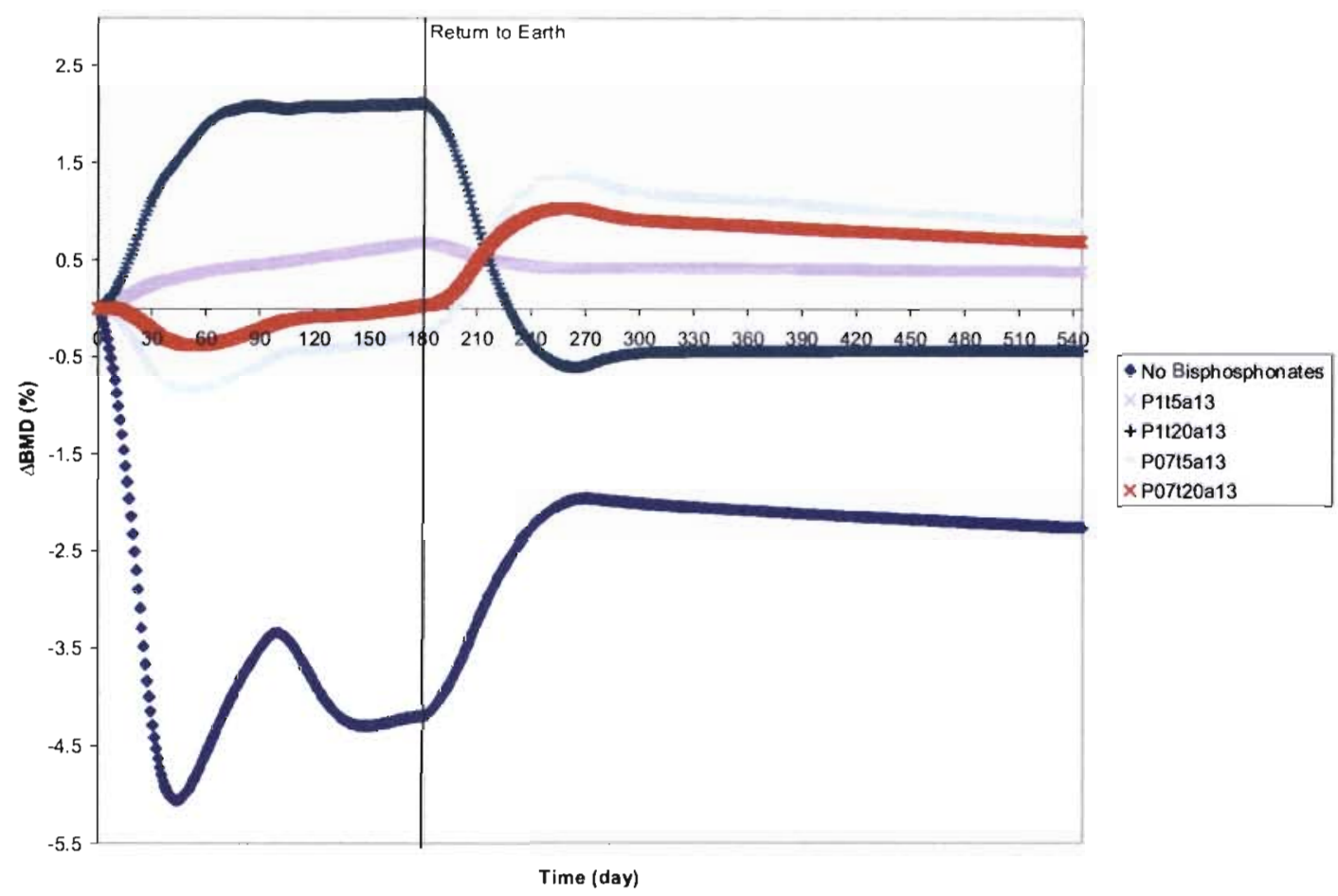

Figure 3.16. Predicted bisphosphonate effects on BMD and posttreatment return to Earth from 180day spaceflight.

Upon return to Earth, predicted damage accumulation increased for treatments given at the beginning of spaceflight that created bone balances of 1.2 (Figure 3.17). This was the opposite case for bone balances of 1.3 , where the model predicted further decreases in damage post-flight and post-treatment (Figure 3.18), except in the case of 365-day spaceflight where predicted damage loss was substantial during flight (Figure 3.19). Prediction results for treatments with high levels of remodeling suppression did not follow these trends, as they always led to decreases in damage during postflight recovery. 


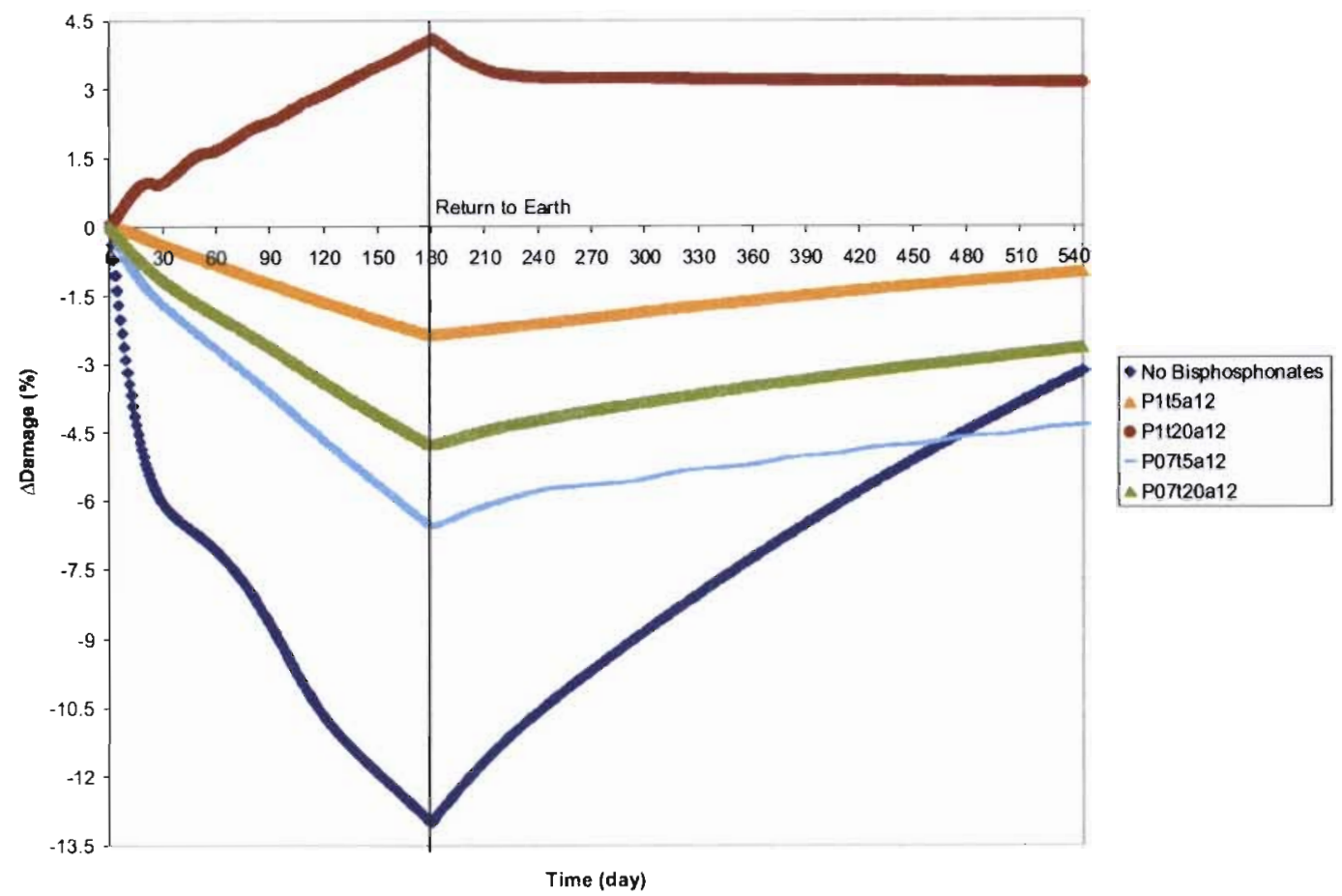

Figure 3.17. Predicted bisphosphonate effects on damage accumulation (D) and posttreatment return to Earth from 180-day spaceflight.

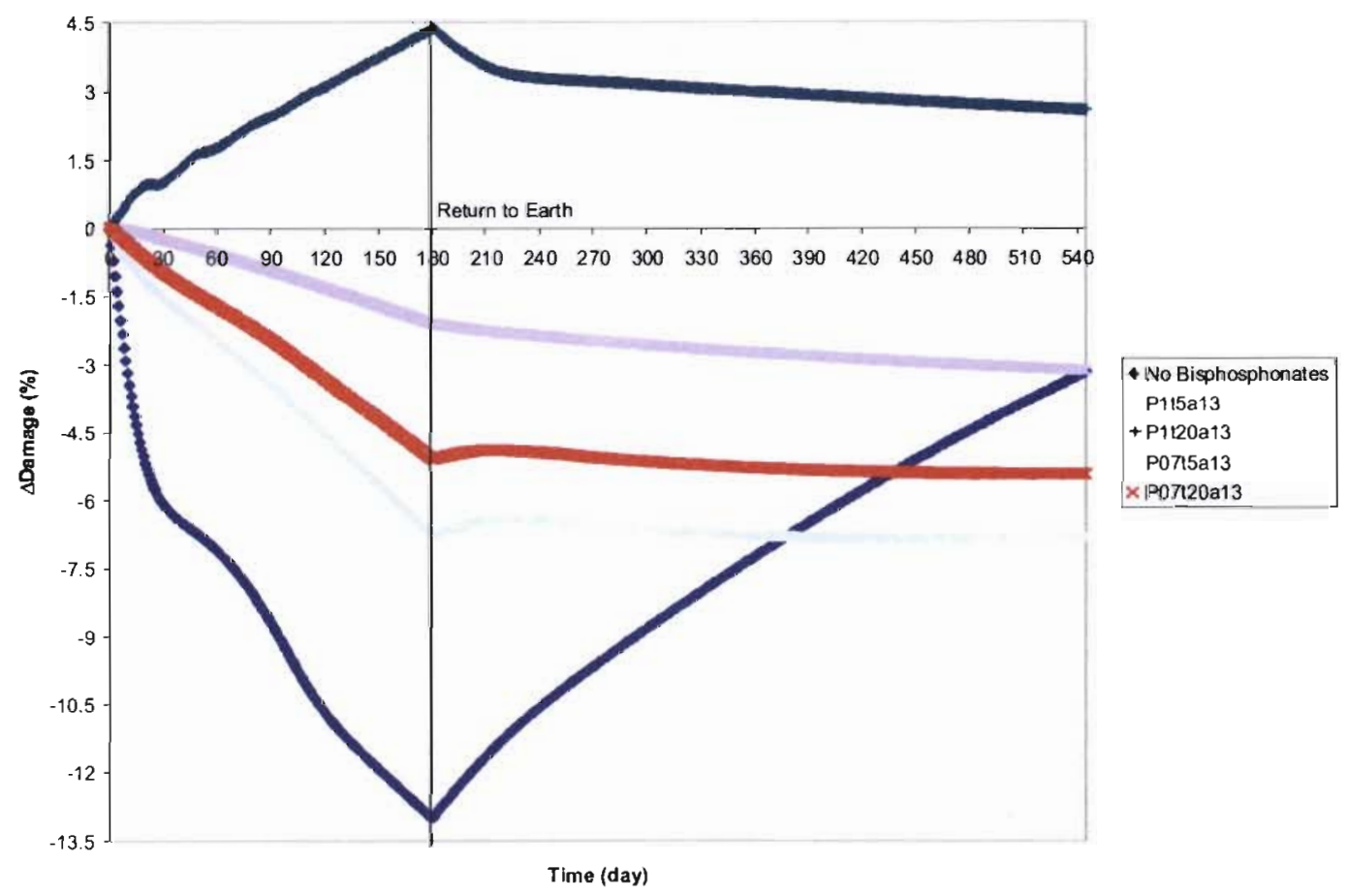

Figure 3.18. Predicted bisphosphonate effects on damage accumulation (D) and posttreatment return to Earth from 180-day spaceflight. 


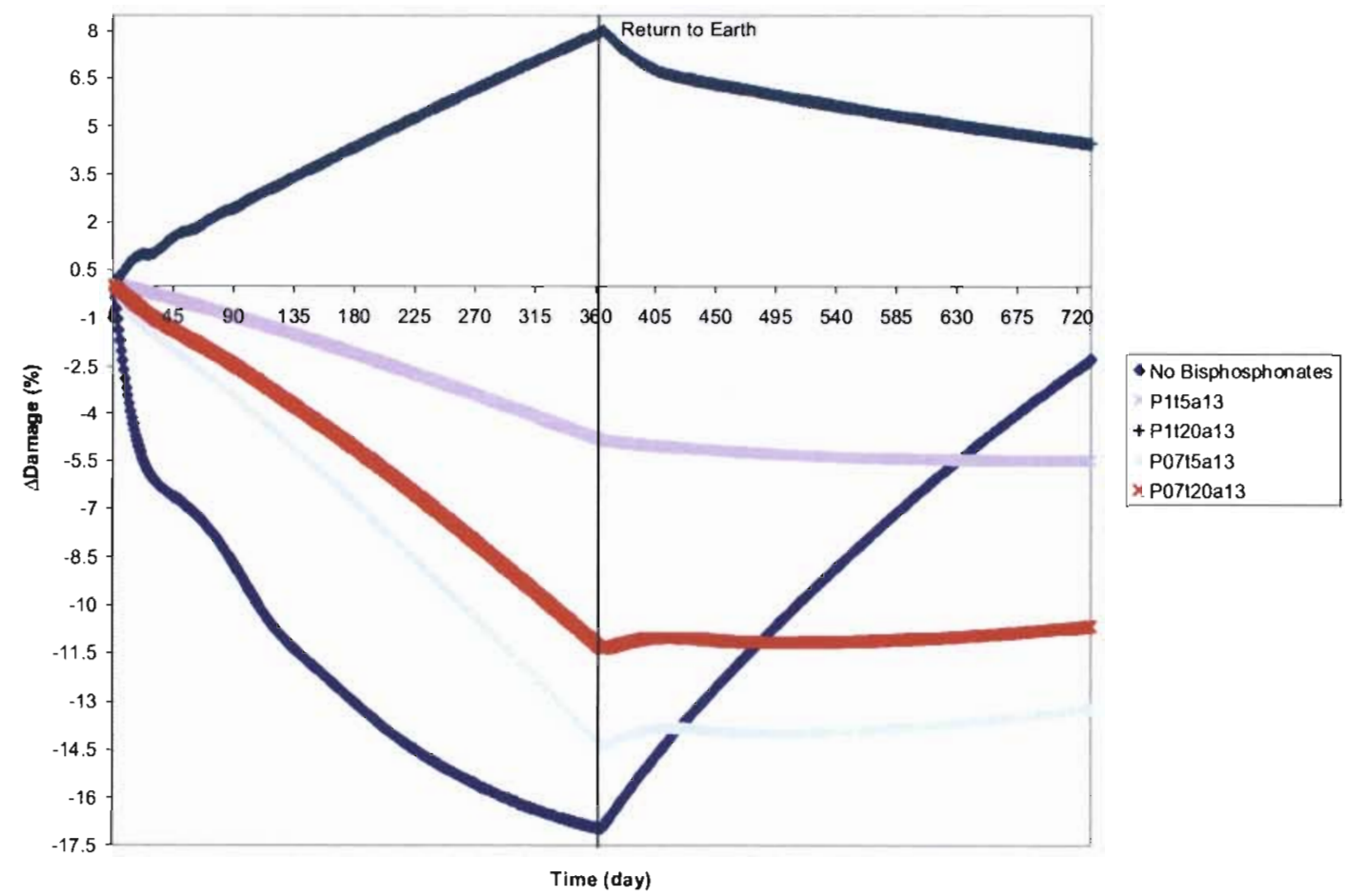

Figure 3.19. Predicted bisphosphonate effects on damage accumulation (D) and posttreatment return to Earth from 365-day spaceflight.

The predicted gains in BMD due to preflight treatment appear to have increased the ability to recover from spaceflight as predicted BMD and damage nearly reach pretreatment values (Figures 3.20 and 3.21 for bisphosphonates given 90 days preflight. See appendices B, C, D, and E for treatment results for 10-, 90-, 180-, and 365-day spaceflights, respectively.). Most of the predicted values for BMD and damage either were near or reached equilibrium after 1-year of postflight recovery. The model predicted 1-year postflight recovery values for BMD and damage to be both above and below pretreatment values, depending on the treatment. 


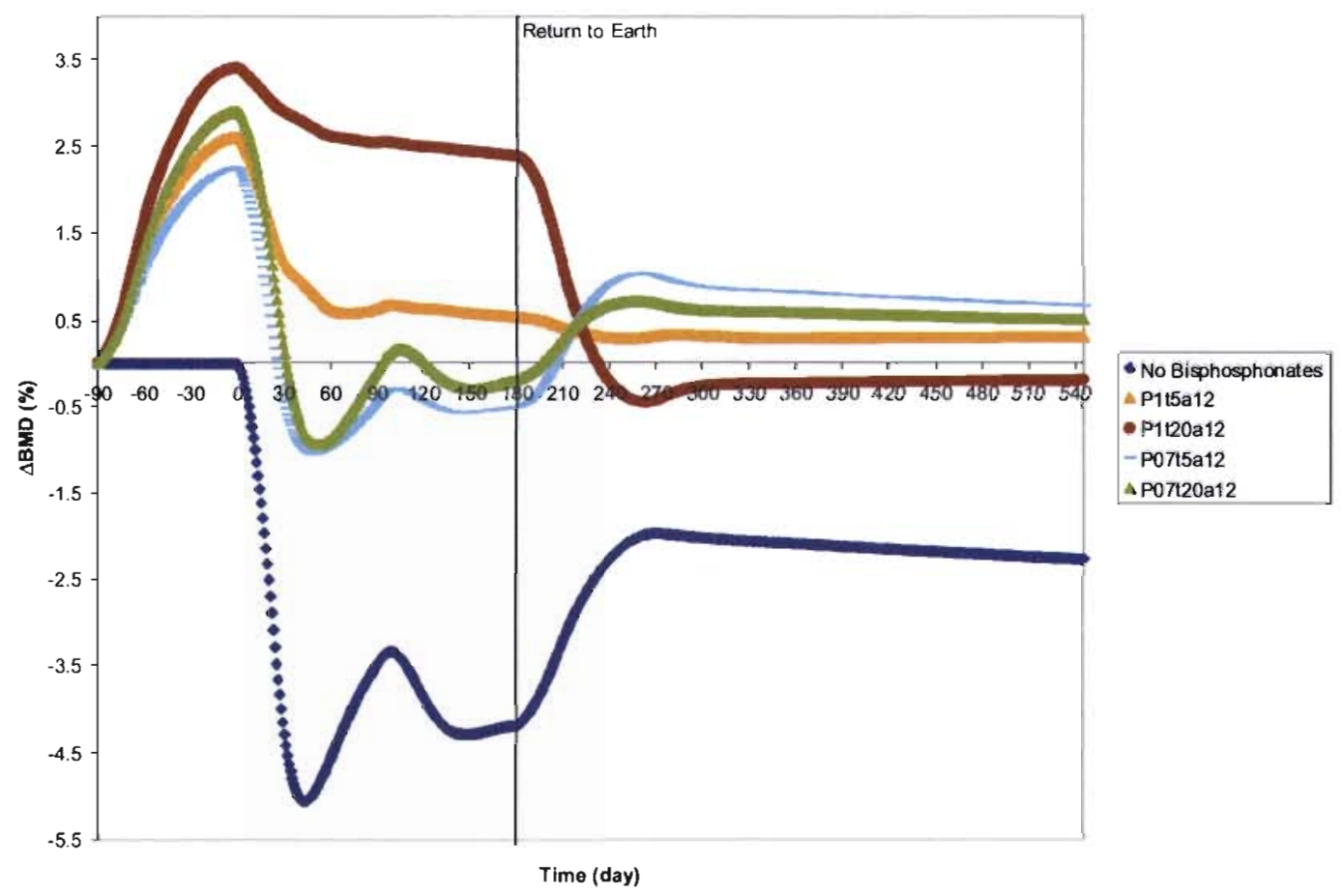

Figure 3.20. Predicted bisphosphonate effects beginning 90 days preflight on BMD and posttreatment return to Earth from 180-day spaceflight.

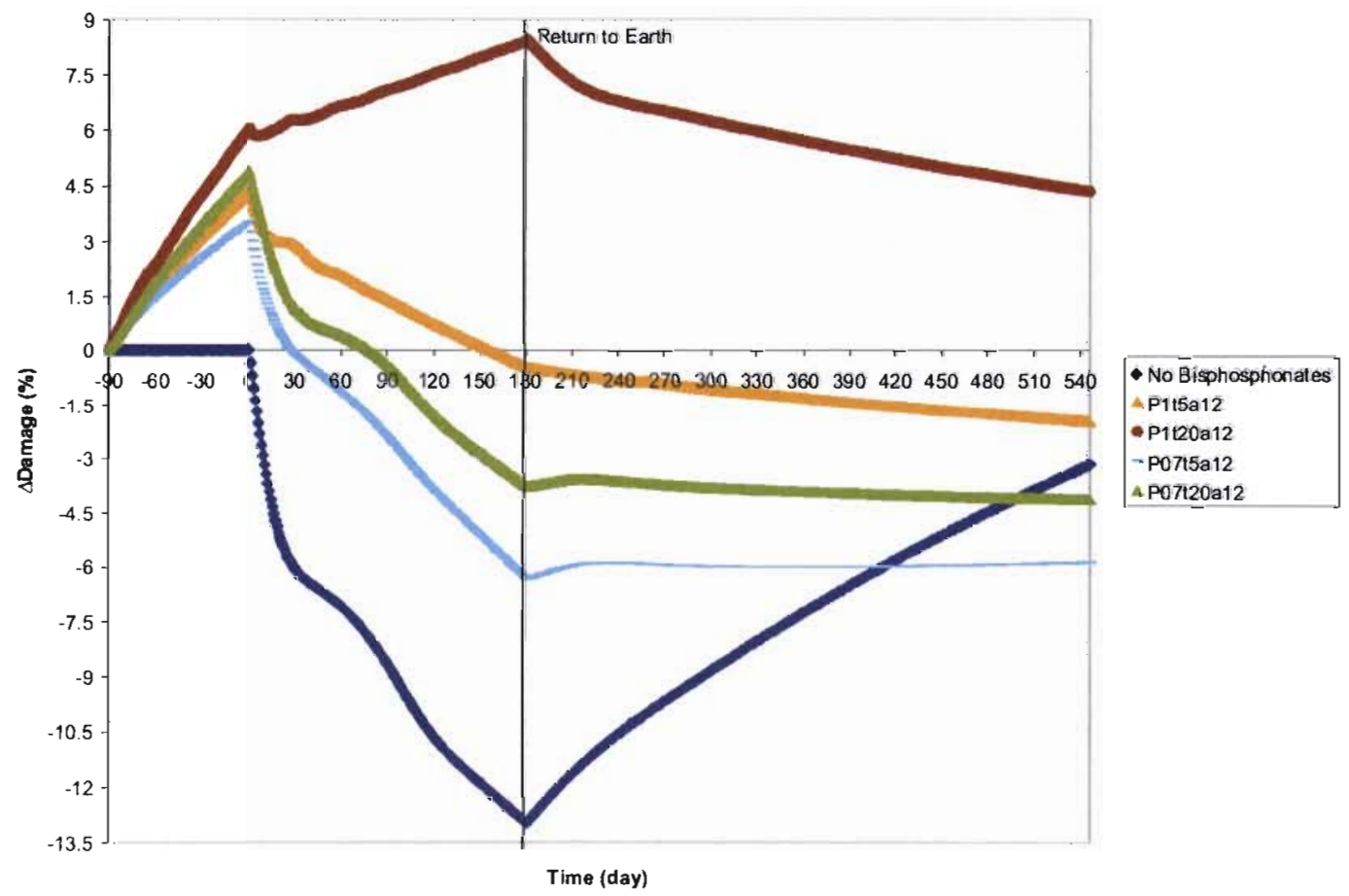

Figure 3.21. Predicted bisphosphonate effects beginning 90 days preflight on damage accumulation (D) and posttreatment return to Earth from 180-day spaceflight. 


\section{CHAPTER 4: DISCUSSION}

The computer model developed here combines previous bone remodeling and bisphosphonate algorithms plus spaceflight data obtained from experimental studies in literature in order to better understand the adverse effects of microgravity on bone and predict potential treatments for space explorers. The model predicted reduced risk of fracture by increasing bone quantity and either increasing or only slightly reducing bone quality for treatments (1) with low to intermediate suppression of remodeling activation and (2) that create higher bone balance ratios. The simulation also predicted significant changes to BMD and damage upon return to Earth as the remodeling response readjusted to higher stress conditions. For treatments highly suppressing remodeling activation, these predicted postflight changes included decreased BMD and increased damage accumulation. Low levels of remodeling suppression led the model to predict substantial increases in BMD and small increases in damage postflight. Postflight changes were minimal for treatments with intermediate suppression.

The model was developed to match the 4.2 percent loss in BMD over 180 days in

space as seen on the International Space Station $[3,5,6]$. The model's greatest predicted BMD loss in untreated, trabecular vertebral bone was 5.01 percent for a 365 -day spaceflight. This is less than half the highest loss (-10.8 percent) seen in Russian cosmonauts on Salyut missions lasting 5 to 7 months [5]. Though the model does not match the results from these older missions, it is likely due to advancements in technology, physical preparedness of subjects, and onboard exercise routines that were developed for missions to the International Space Station. 
Full recovery for space explorers returning from the ISS took from 1 to 3 years to complete [5]. The model developed here predicted that full recovery to preflight BMD values may never be attained without treatment. With bisphosphonate treatment, the model predicted complete recovery to occur; some treatments even resulted in higher BMD values than existed preflight. The model suggests that bisphosphonate treatment, combined with exercise, may be the solution that NASA and other space exploration programs desire to combat bone deterioration in space.

The predicted remodeling response of untreated bone to environmental changes was non-linear, as most BMD was lost or gained early on in the transitions from Earth to space and space to Earth. For 10 days in space, the model predicted more mineral to be lost while readjusting to Earth's gravity than lost during spaceflight. This has yet to be examined experimentally, but certainly the model provided insight into a possible trend that may have gone unnoticed. Knowing when fracture risk is maximized may provide for better postflight recovery programs so that fracture can be avoided.

The model predicted bisphosphonate treatment to be beneficial for all durations of spaceflight, not just for longer duration missions. In many instances, preflight treatments were shown to reduce the fracture risk upon return to Earth. Longer simulated flight durations required longer preflight treatments to provide similar effects to those with shorter flights and shorter pretreatments. The problem with this is that as treatment on Earth is lengthened, damage accumulation increases to such an extent that it could actually cause an increase in fracture risk before entering space. Based on the model's predictions of damage increase, pretreatment periods longer than 30 days may put the subjects at risk. During pretreatment, the subjects are still on Earth where higher stresses 
cause greater increases in damage as compared to space. They may also be exposed to even higher stresses due to exercises in preparation for the mission. These exercises, combined with brittler bones, could lead to a fracture before flight and put a hold on the mission.

Most interestingly, the model predicted treatments with high suppression of remodeling activation to have the highest gain in BMD at the end of flight, and the lowest BMD values 1 -year postflight. These treatments almost completely inhibited remodeling, causing large amounts of damage to accumulate. Though BMD was much higher, the quality of bone was poor. Upon return to earth and discontinuation of treatment, bone remodeling was no longer inhibited and responded to the high amount of damage. Damage and bone were removed at a much greater rate than bone formation occurred, causing quality of bone to increase, but quantity to decrease.

Alternately, the model predicted treatments with low suppression of activation frequency to have the lowest BMD at the end of spaceflight, but they had the highest BMD and lowest amount of damage 1-year postflight. These treatments allowed a fair amount of remodeling to continue in space, but limited it enough so that bone loss was kept to a minimal amount. A majority of the predicted bone loss came from lost damage, so upon return to earth damage accumulation did not activate a remodeling response. These treatments had the largest postflight gains in BMD because the response was mostly due to loading in which bone was added to meet the strength required to support the subject.

Treatments with intermediate suppression and bone balance ratios of 1.3 were optimal for both end-of-flight and 1-year postflight. The model predicted these treatments 
to lower risk of fracture both upon return to Earth and after 1 year of recovery. Lower levels of suppression allowed just enough resorption to remove a good amount of fatigue microdamage and increase bone quality, while higher bone balances appropriated more formation than resorption, increasing bone mass.

Since the simulation of bisphosphonate treatment in this model was short in comparison with other models, it is difficult to determine if limits of BMD growth were reached. Predicted BMD gains were non-linear and fluctuated throughout the simulation; in contrast, previous models that did not account for damage and disuse stimuli showed only permanent, linear gain in mass $[32,33,34]$.

Similar to Lacy's model of trabecular bone turnover [34], activation frequency was found to have the greatest effect on bone mass. Unloading in microgravity caused a disuse response in untreated bone, increasing BMU activation and leading to the resorption of large amounts of bone. The model by Hernandez et al. [33] also showed similar results in that the initial gain in bone mass was dependent upon the level of remodeling suppression and bone balance ratio. Heaney's model [32] exhibited an initial gain dependent upon the pretreatment remodeling parameters; however this model did not examine variations in these parameters before treatment.

It is difficult to compare the accumulation of damage of this model to others since it also simulates microgravity. The model by Nyman et al. [23] predicted small gains in microdamage initially that were proportional to activation frequency suppression. In contrast, the model developed here predicted losses in microdamage for treatments with low and intermediate levels of suppression and gains in damage accumulation when activation frequency was highly suppressed. 
The limitations of this model occur where assumptions have been made due to the lack of available information. First, the damage rate coefficient, $k_{D}$, was kept constant throughout the simulation, though it is likely to change in space or for various bone balances. Other coefficients, too, such as the damage rate exponent or the activation frequency coefficients, are likely to be altered in space and would benefit from further study of bone remodeling in microgravity. Second, the predicted postflight results are limited by the fact that they are based on the same stress applied preflight even though postflight recovery programs enable space explorers to ease back into full loading. This high postflight stress would cause overpredictions of both BMD and damage. Also, a bone balance ratio of 1.0 was instantly applied upon return to Earth, when it is more likely that the ratio would slowly ease back down. Third, the simulation applies a constant stress derived from bone loss to a section of bone rather than deriving the actual strain and applying it to a finite element model. Using a finite element model would create a more accurate remodeling response with more precise loading conditions and allow detailed analysis of the effects of specific exercises on maintaining bone mass. Lastly, the model does not take into account effects of spaceflight on blood flow, drug metabolism, tissue binding, drug elimination, fluid shear stress, or changes in hormone levels $[2,4]$. Many of these affect the efficacy of the drug itself. Changes to fluid shear stress in a microgravity environment could affect the mechanosensory ability of osteoclasts to sense signals indicating bone loading and would lead to further loss of bone even under heavy exercise [4]. Also, although the model tracks changes to the populations of osteoblasts and osteoclasts, it only accounts for changes due to the 
remodeling response and not due to the physiological adaptations that may occur in microgravity [2].

Changes in hormone levels or physiological alterations to the populations of osteoblasts or osteoclasts could significantly alter the remodeling response in space and the response to bisphosphonate treatment [4]. With reduced levels of PTH, IGF-1, and growth hormone [4], it would be likely for remodeling formation and resorption rates to be altered in microgravity. With so little bone formation occurring, it could also be possible that active osteoclasts may significantly outnumber osteoblasts and lead to a slower recovery response upon return to earth.

It is clear that the model would significantly benefit from further studies on spaceflight. Though the model has to overcome the many unknown variables of bone remodeling, bisphosphonates, and microgravity, it has shown the ability to provide potential trends for future studies. As new data and information becomes available, the model's accuracy can only be improved and could eventually be a tool used for predicting effects of other treatments as well. 


\section{SUMMARY OF CONCLUSIONS}

- The model predicted bisphosphonates reduced fracture risk by increasing bone quantity and either increasing or only slightly reducing bone quality for treatments:

- (1) with low to intermediate suppression of remodeling activation

- (2) that create higher bone balance ratios

- Most changes to BMD occurred early on when adjusting to new environments

- Predicted BMD loss was fairly consistent with data from missions to the International Space Station

- Preflight treatments were shown to reduce the risk of fracture for all durations of spaceflight

- Longer preflight treatment periods may put the subject at risk due to increased microdamage accumulation

- Overall, the model suggested that bisphosphonate treatment, combined with existing exercise programs, may be the solution to combat bone deterioration in space 


\section{REFERENCES}

[1] Payne MWC, Williams DR, Trudel G. Space flight rehabilitation. Am J of Phys Med Rehabil 2007;86:583-91

[2] Shapiro, JR. Microgravity and drug effects on bone. J. Musculoskelet Neuronal Interact 2006;6:322-23.

[3] Lang T, LeBlanc A, Evans H, Lu Y, Genant H, Yu A. Cortical and trabecular bone mineral loss from the spine and hip in Long-Duration Spaceflight. J Bone Miner Res 2004;19:1006-12.

[4] Bloomfield, SA. Summary - bone in microgravity environments: "Houston, we have a problem." J Musculoskelet Neuronal Interact 2006;6:329-30.

[5] LeBlanc AD, Spector ER, Evans HJ, Sibonga JD. Skeletal responses to space flight and the bed rest analog: a review. J Musculoskelet Neuronal Interact 2007;7:33-47.

[6] Iwamoto J, Takeda T, Sato Y. Interventions to prevent bone loss in astronauts during space flight. Keio J Med 2005;54:55-9.

[7] Martin RB, Burr DB, Sharkey NA. Skeletal Tissue Mechanics. New York: Springer; 1998.

[8] Gunaratne G. A theoretical analysis of vibrational modes aimed at their use as measures of bone damage. ISSO Annual Report 2005:105-7,126.

[9] Epstein S. The roles of bone mineral density, bone turnover, and other properties in reducing fracture risk during antiresorptive therapy. Mayo Clin Proc 2005;80:379-88.

[10] Parfitt AM. Bone age, mineral density, and fatigue damage. Calcified Tissue International 1993;53(Supplement 1):S82-6.

[11] Osteoblasts and Osteoclasts. 2004. Children's Hospital Boston. 1 June 2008. $<$ http://www.childrenshospital.org/cfapps/research/data_admin/Site31/mainpageS 31P1 sublevel2.html>

[12] Bentolila V, Boyce TM, Fyhrie DP, Drumb R, Skerry TM, Schaffler, MB. Intracortical remodeling in adult rat long bones after fatigue loading. Bone $1998 ; 23: 275-81$.

[13] Burr DB, Martin RB, Schaffler MB, Radin EL. Bone remodeling in response to in vivo fatigue microdamage. J Biomech 1985;18:189-200.

[14] Burr DB, Martin RB. Calculating the probability that microcracks initiate resorption spaces. J Biomech 1993;26:613-6. 
[15] Mori S, Burr DB. Increased intracortical remodeling following fatigue damage. Bone 1993;14:103-9.

[16] Li XJ, Jee WSS, Chow SY, Woodbury DM. Adaptation of cancellous bone to aging immobilization in the rat: a single photon absorptiometry and histomophometry study. The Anatomical Record 1990;227:12-24.

[17] Chaffler MB, LI XJ. Immobilization induced bone loss: quantitive histological studies of cortical bone resorption. Transactions of the $36^{\text {th }}$ Annual Meeting of the Orthopaedic Research Society 1990;15:187.

[18] Hazelwood SJ, Martin RB, Rashid MM, Rodrigo, JJ. A mechanistic model for internal bone remodeling exhibits different dynamic responses in disuse and overload. J Biomech 2001;34:299-308.

[19] Cullen DM, Smith RT, Akhter MP. Bone-loading response varies with strain magnitude and cycle number. J Appl Physiol 2001;91:1971-6.

[20] Lin JH. Bisphosphonates: a review of their pharmacokinetic properties. Bone 1996;19:80-100.

[21] Rogers MJ, Watts DJ, Russell RG. Overview of bisphosphonates. Cancer 1997;80:1652-60.

[22] Sato M, Grasser W, Endo N, Akins R, Simons H, Thompson DD, et al. Bisphosphonate action. Alendronate localization in rat bone and effects on osteoclast ultrastructure. J Clin Invest 1991;88:2095-105.

[23] Nyman JS, Yeh OC, Hazelwood SJ, Martin RB. A theoretical analysis of longterm bisphosphonate effects on trabecular bone volume and microdamage. Bone 2004;35:296-305.

[24] Fleish H. Bisphosphonates: mechanisms of action. Endocr Rev 1998;19:80-100.

[25] Kimmel DB. Mechanism of action, pharmacokinetic and pharmacodynamic profile, and clinical applications of nitrogen-containing bisphosphonates. J Dent Res 2007;86:1022-33.

[26] Rodan GA, Fleisch HA. Bisphosphonates: mechanisms of action. J Clin Invest 1996;97:2692-6.

[27] Lieberman Ua, Weiss Sr, Broll J, Minne HW, Quan H, Bell NH, RodriguesPortales J, Downs RD Jr, Dequeker J, Favus M. Effect of oral alendronate on bone mineral density and the incidence of fractures in postmenopausal osteoporosis. The Alendronate Phase III Osteoporosis Treatment Study Group. N Engl J Med 1995;333(22):1437-43. 
[28] Tonino RP, Meunier PJ, Emkey R, Rodriguez-Portales JA, Menkes CJ, Wasnich RD, Bone HG, Santora AC, WU m, Desai R, Ross PD. Skeletal benefits of alendronate: 7-year treatment of postmenopausal osteoporotic women. Phase III Osteoporosis Treatment Study Group. J Clin Endocrinol Metab 2000;85:109-15.

[29] Mashiba T, Turner CH, Hirano T, Forwood MR, Johnston CC, Burr DB. Effects of suppressed bone turnover by bisphosphonates on microdamage accumulation and biomechanical properties in clinically relevant skeletal sites in beagles. Bone 2001;28:524-31.

[30] Carter DR, Fyhrie DP, Whalen RT. Trabecular bone density and loading history: regulation of connective tissue biology by mechanical energy. J Biomech 1987;20:785-94.

[31] Huiskes R, Weinans H, Grootenboer HJ, Dalstra M, Fudala B, Slooff TJ. Adaptive bone-remodeling theory applied to prosthetic-design analysis. $\mathrm{J}$ Biomech 1987;20:1135-50.

[32] Heaney RP, Yates AJ, Santora II AC. Bisphosphonate effects and the bone remodeling transient. J Bone Miner Res 1997;12:6-15.

[33] Hernandez CJ, Beaupré GS, Marcus R, Carter DR. A theoretical analysis of the contributions of remodeling space, mineralization, and bone balance to changes in bone mineral density during alendronate treatment. Bone 2001;29:511-6.

[34] Lacy ME, Bevan JA, Boyce RW, Geddes AD. Antiresorptive drugs and trabecular bone turnover: validation and testing of a computer model. Calcif Tissue Int 1994;54:179-85.

[35] Currey JD. The effect of porosity and mineral content on the Young's modulus of elasticity of compact bone. J Biomech 1988;21:131-9.

[36] Rho JY, Ashman RB, Turner CH. Young's modulus of trabecular and cortical bone material: ultrasonic and microtensile measurements. J Biomech 1993;26:111-9.

[37] Turner CH, Anne V, Pidaparti RMV. A uniform strain criterion for trabecular bone adaptation: do continuum-level strain gradients drive adaptation? J Biomech 1997;30:555-63.

[38] Hart RT, Davy DT. Theories of bone modeling and remodeling. In: Cowin SC, editor. Bone Mechanics. Boca Raton, FL: CRC Press; 1989, pp.253-77.

[39] Martin RB. The usefulness of mathematical models for bone remodeling. Yearbook of Physical Anthropology 1985;28:227-36. 
[40] Parfitt AM. The physiologic and clinical significance of bone histomorphometric data. In: Recker RR, editor. Bone Histomorphemtry: Techniques and Interpretation. Boca Raton, FL: CRC Press; 1983, pp.143-223.

[41] Frost HM. On rho, a marrow mediator, and estrogen: their roles in bone strength and "mass" in human females, osteopenias, and osteoporoses - insights from a new paradigm. J Bone Miner Metab 1998;16:113-23.

[42] Martin RB. Porosity and specific surface of bone. Critical Reviews in Biomedical Engineering 1984;10:179-222.

[43] Frost HM. Tetracycline-based histological analysis of bone remodeling. Calcif Tissue Res 1969;3:211-37.

[44] Martin RB. Mathematical model for repair of fatigue damage and stress fracture in osteonal bone. J Orthop Res 1995;13:309-16.

[45] Chappard D, Minaire P, Privat C, Berard E, Mendoza-Sarmiento J, Tournebise H, Basle MF, Audran M, Rebel A, Picot C, Gaud C. Effects of tiludronate on bone loss in paraplegic patients. J Bone Miner Res 1995;10:112-8.

[46] Rapillard L, Charlebois M, Zysset K. Compressive fatigue behavior of human vertebral trabecular bone. J Biomech 2006;39:2133-9.

[47] Whalen RT, Carter DR, Steele CR. Influence of physical activity on the regulation of bone density. J Biomech 1988;21:825-37.

[48] Schaffler MB, Choi K, Milgrom C. Aging and matrix microdamage accumulation in human compact bone. Bone 1995;17:521-25.

[49] Brockstedt H, Kassem M, Eriksen EF, Mosekilde L, Melsen F. Age- and sexrelated changes in iliac cortical bone mass and remodeling. Bone 1993;14:681-91.

[50] Hernandez CJ, Keaveny TM. A biomechanical perspective on bone quality. Bone 2006;39:1173-81.

[51] Morgan EF, Yeh OC, Keaveny TM. Damage in trabecular bone at small strains. Eur J Morphol 2005;42:13-21.

[52] Ammann P, Rizzoli R. Bone strength and its determinants. Osteoporos Int 2003;14:S13-8. 


\section{APPENDIX A: MATLAB CODE}

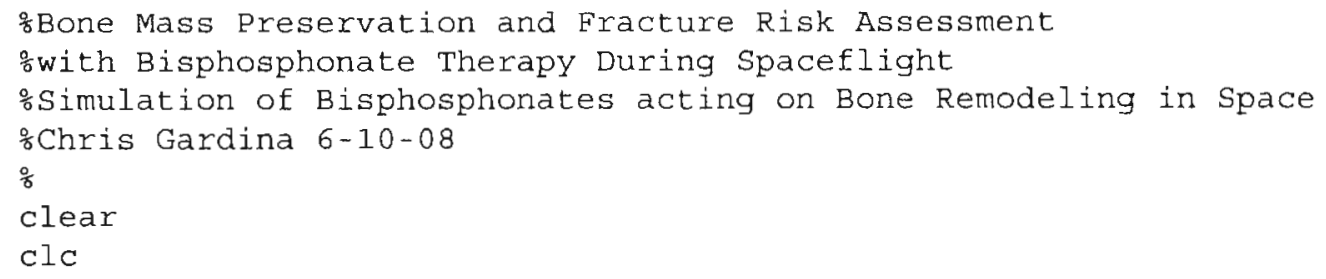

\% Cross-sectional area of bone 


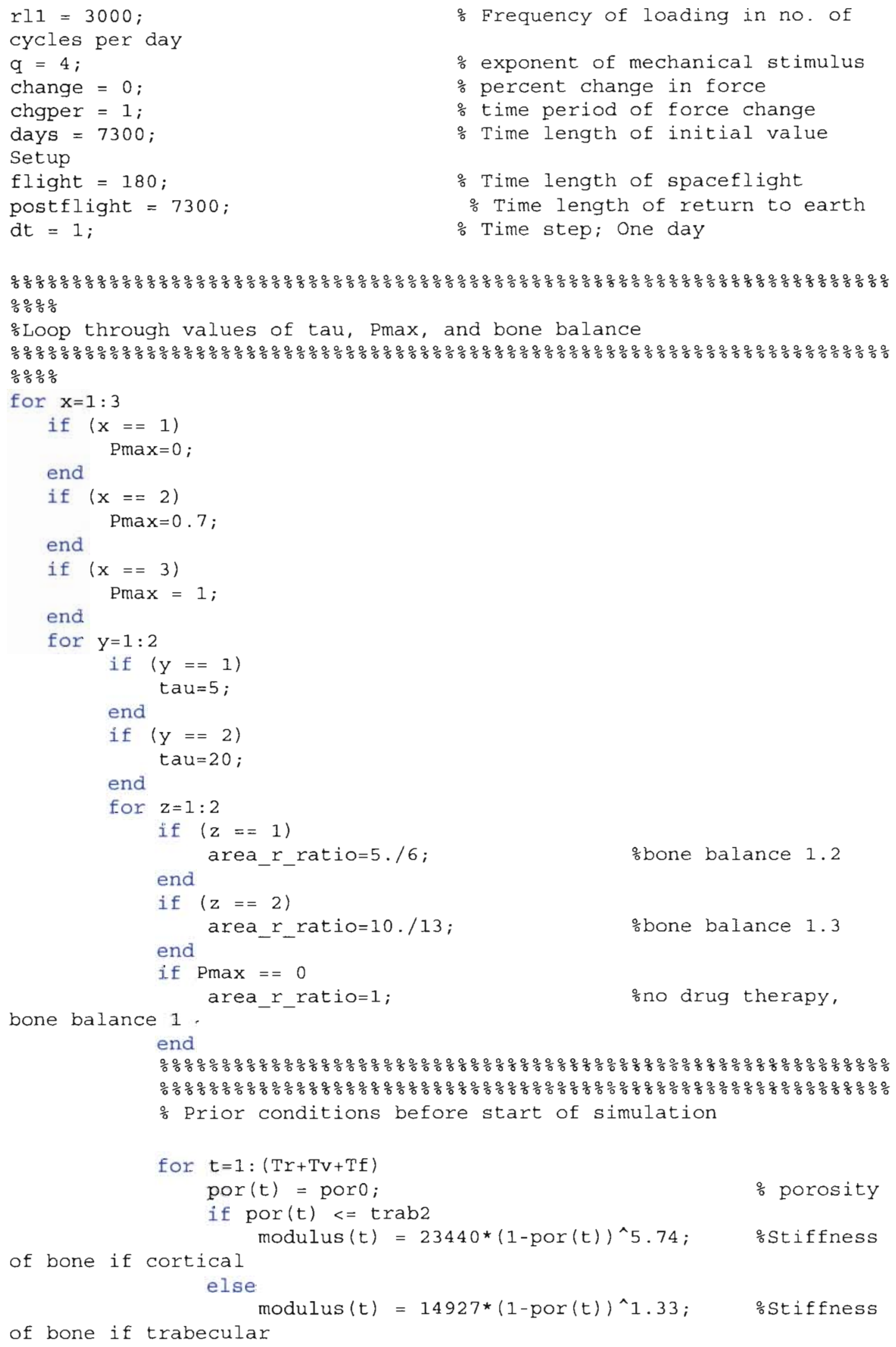




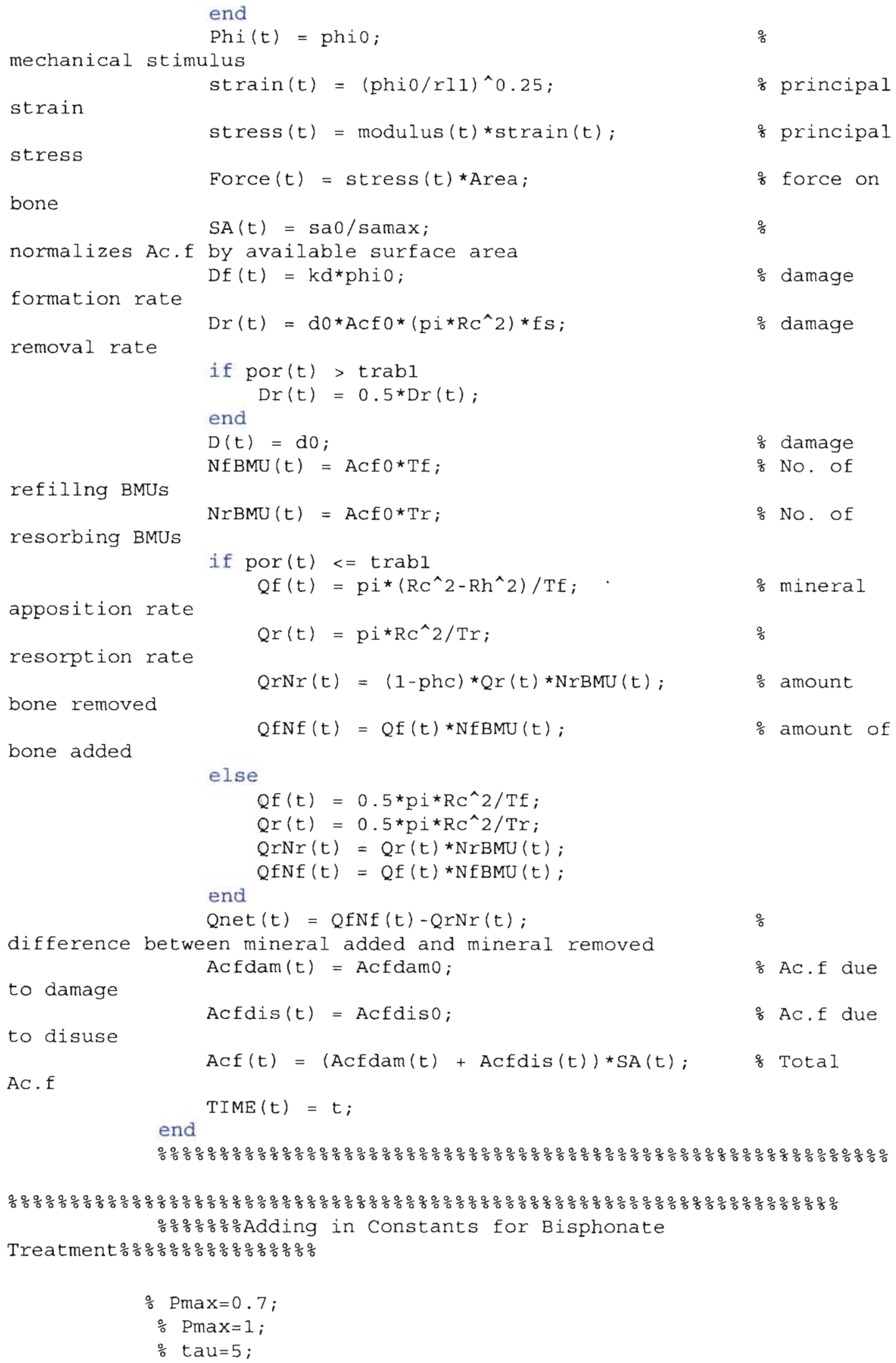




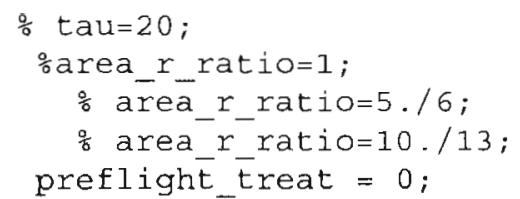

\%bone balance 1

obone balance 1.2

obone balance 1.3

DDays of Bisphosphonate

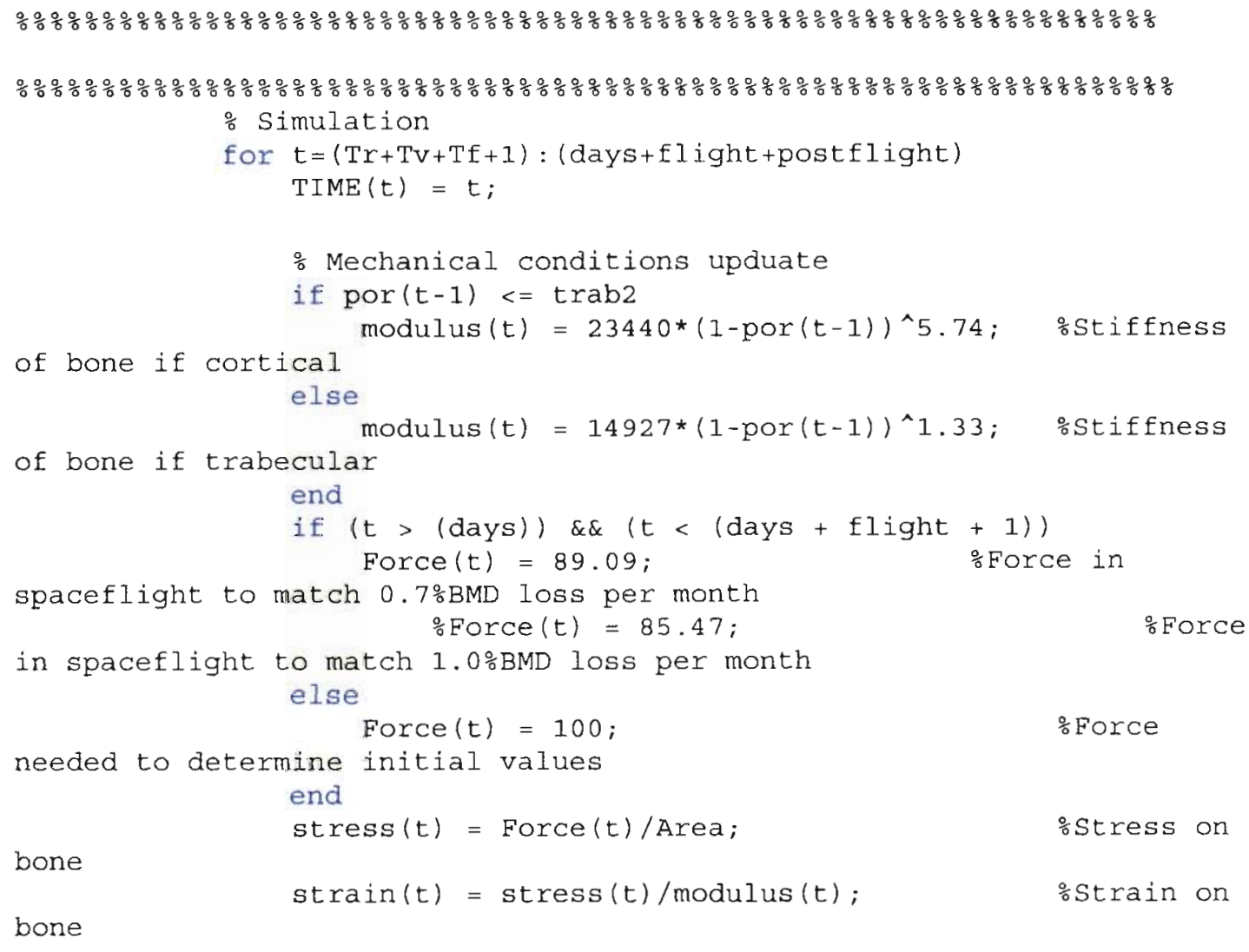




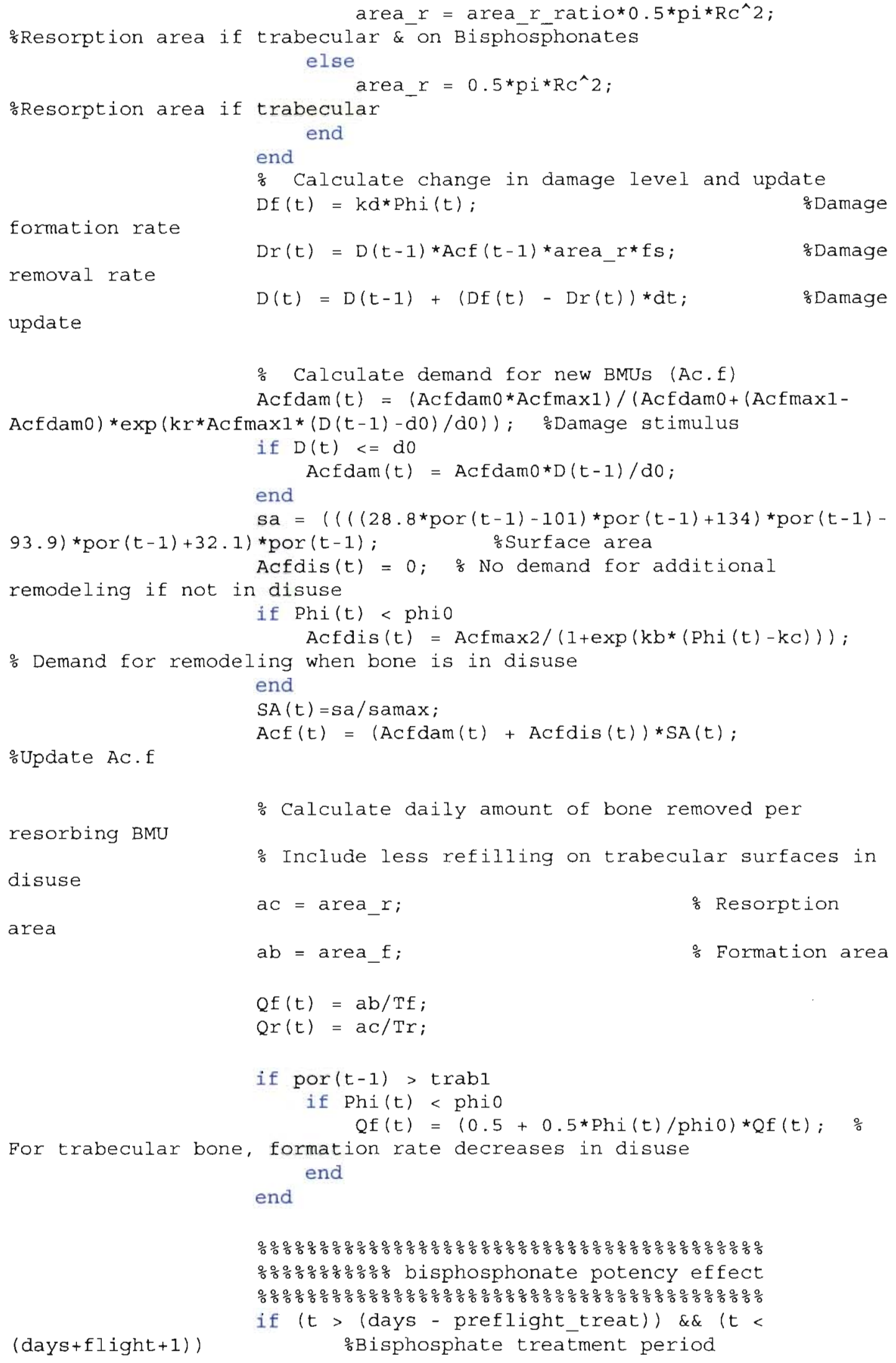




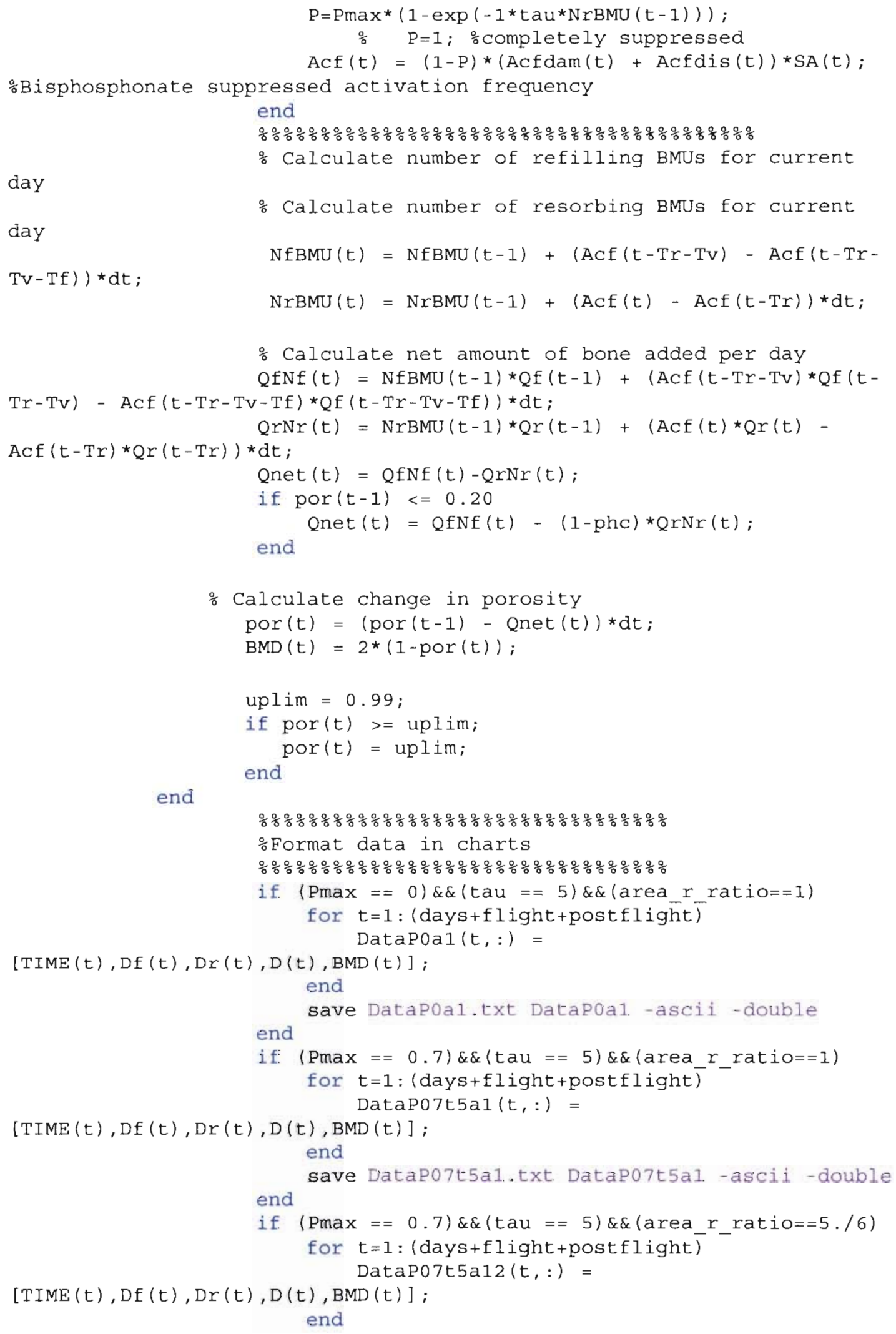


double

save DataP07t5al2.txt DataPo7t5al2 -ascii -

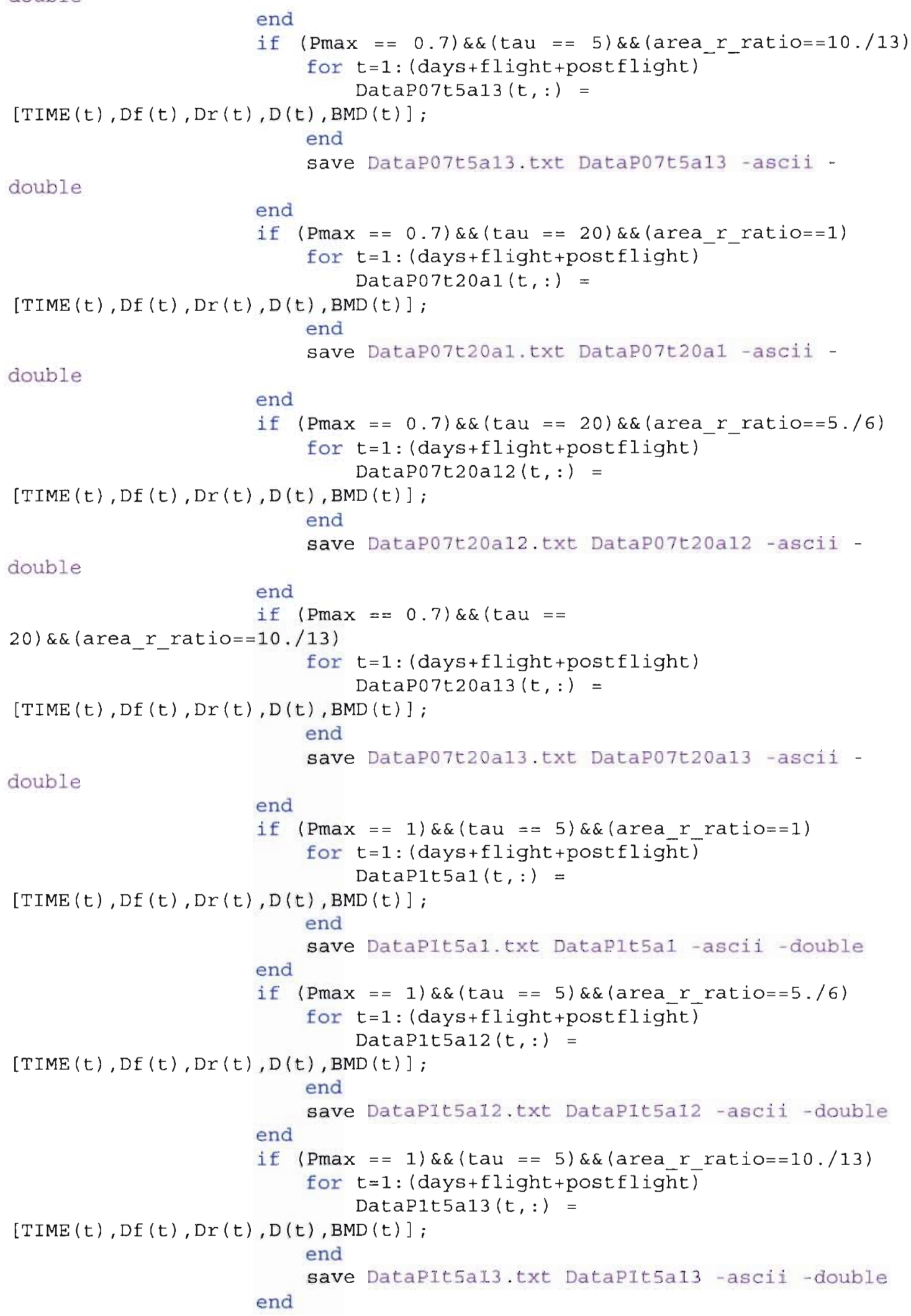


if. $(\operatorname{Pmax}==1) \& \&($ tau $==20) \& \&($ area_r_ratio $==1)$

for $t=1:$ (days+flight+postflight)

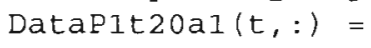

end 


\section{APPENDIX B: FIGURES (10-DAY SPACEFLIGHT)}

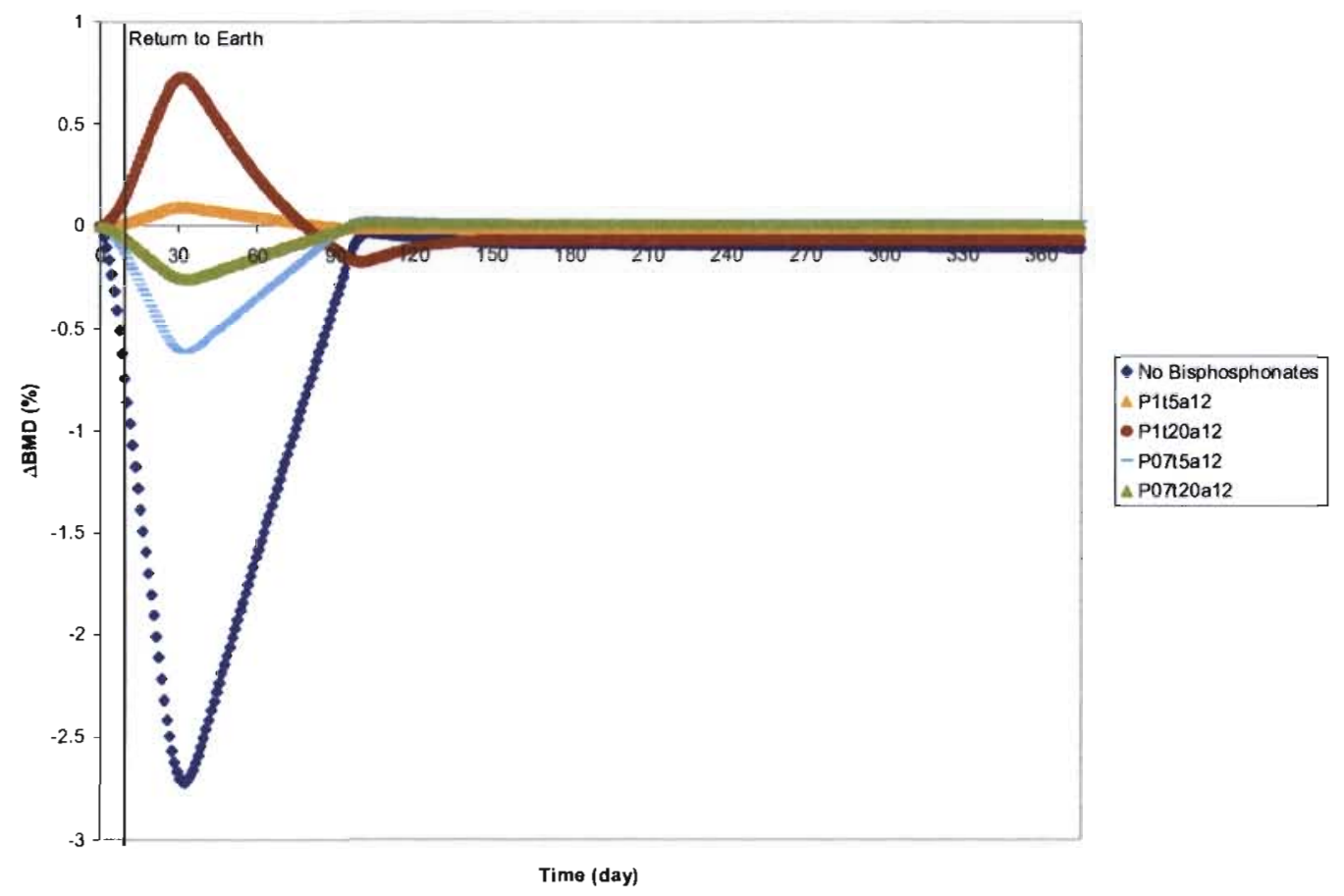

Figure B1. Predicted bisphosphonate effects on BMD and posttreatment return to Earth from 10-day spaceflight.

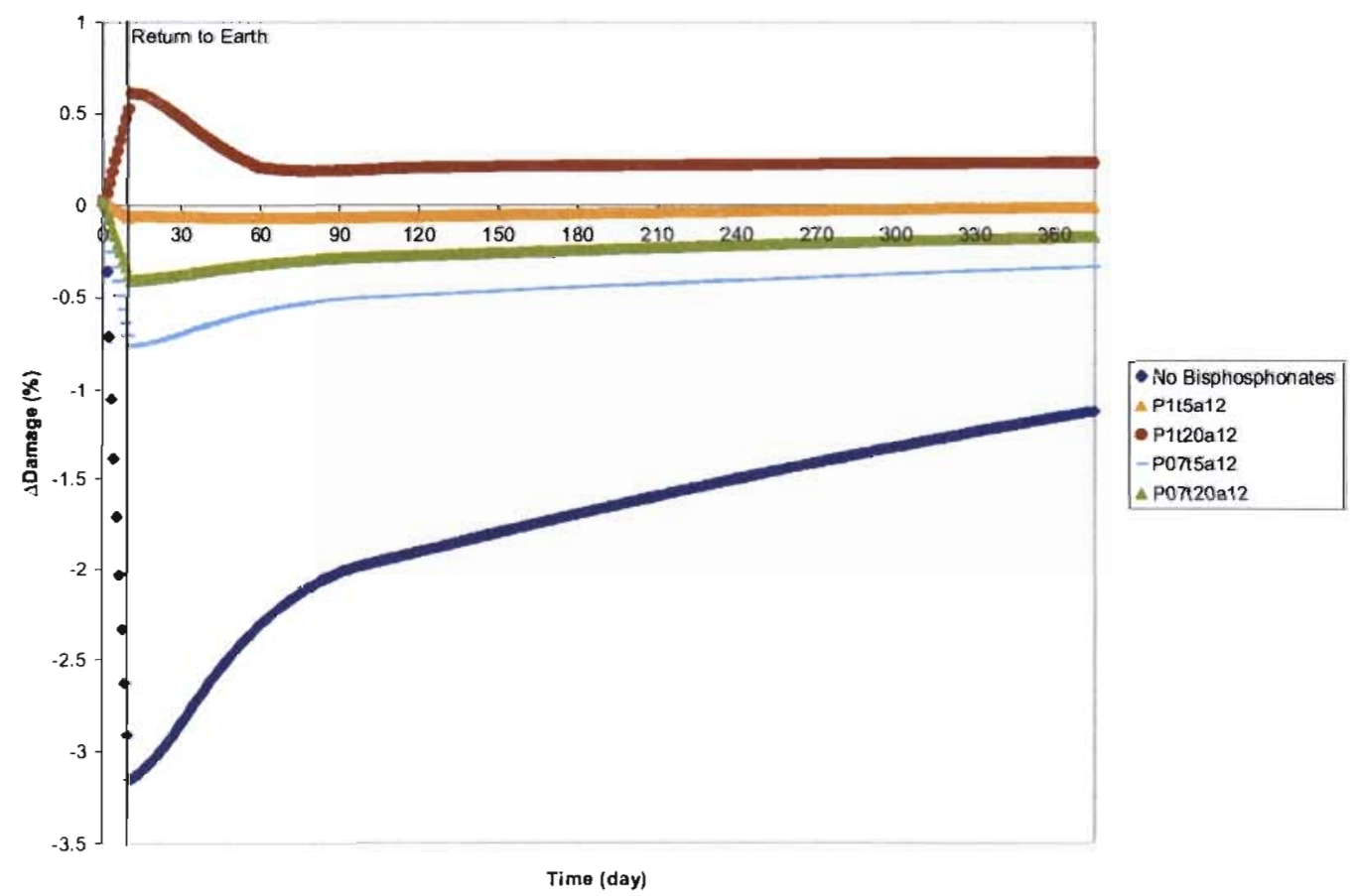

Figure B2. Predicted bisphosphonate effects on damage accumulation (D) and posttreatment return to Earth from 10-day spaceflight. 


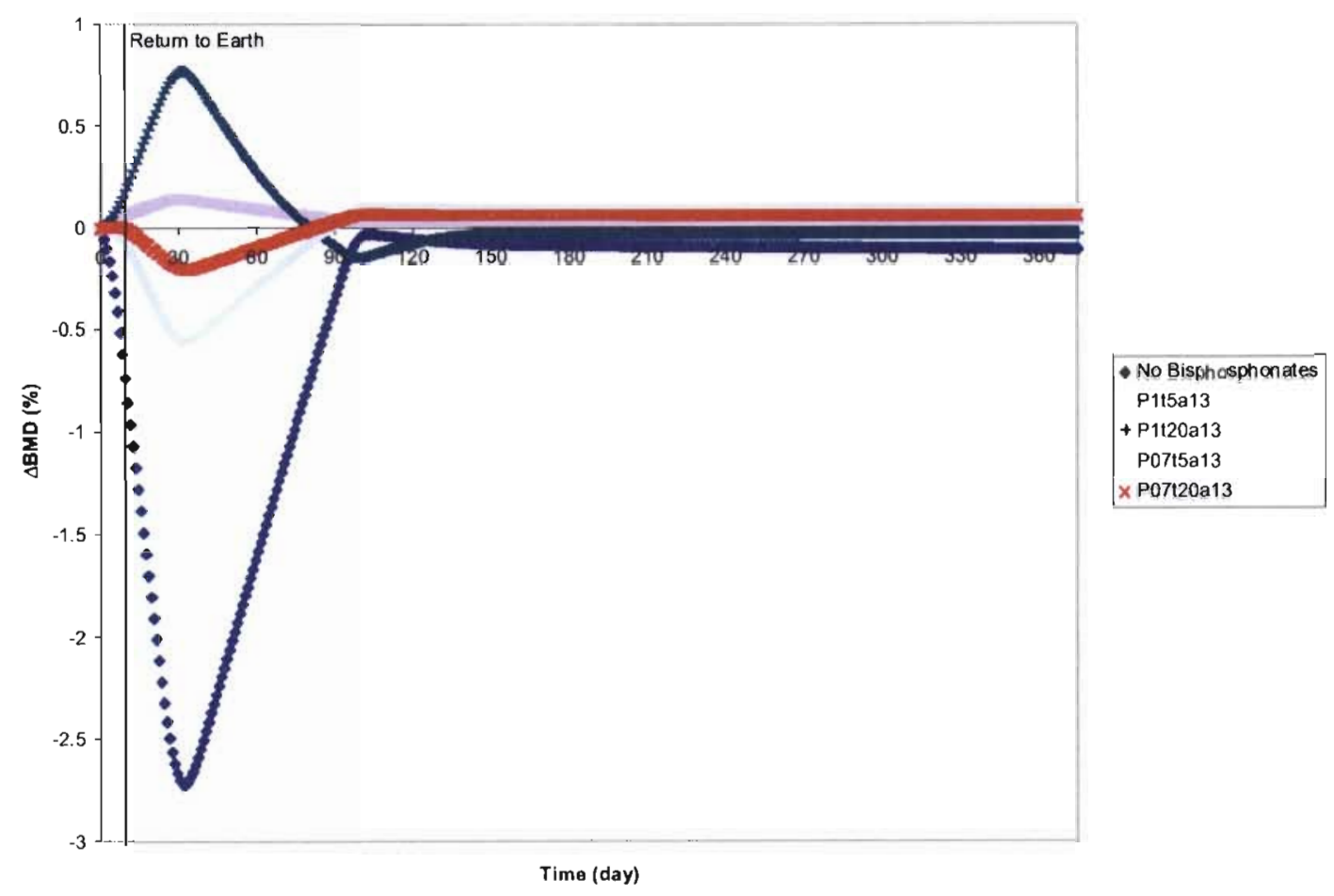

Figure B3. Predicted bisphosphonate effects on BMD and posttreatment return to Earth from 10-day spaceflight.

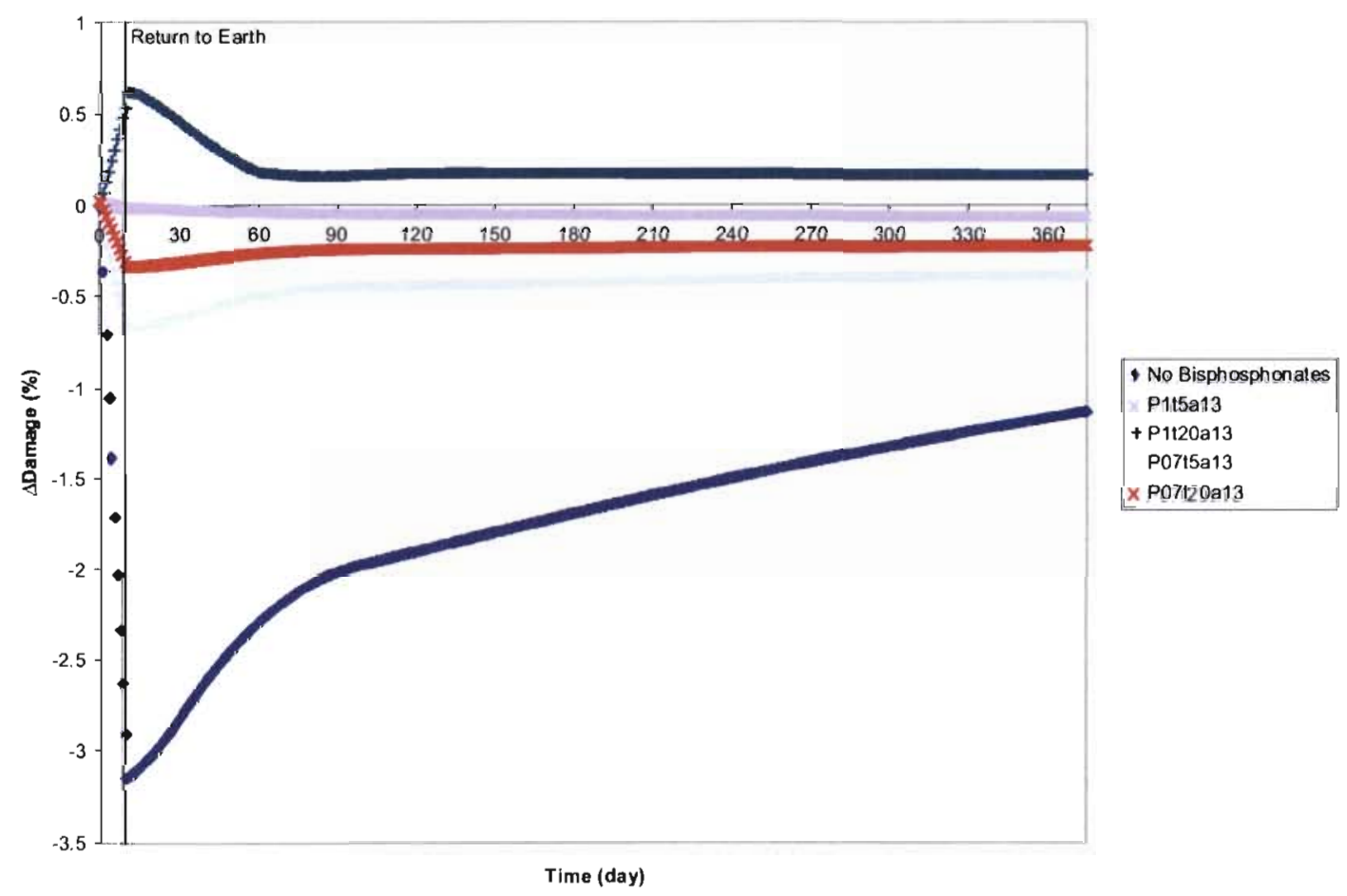

Figure B4. Predicted bisphosphonate effects on damage accumulation (D) and posttreatment return to Earth from 10-day spaceflight. 


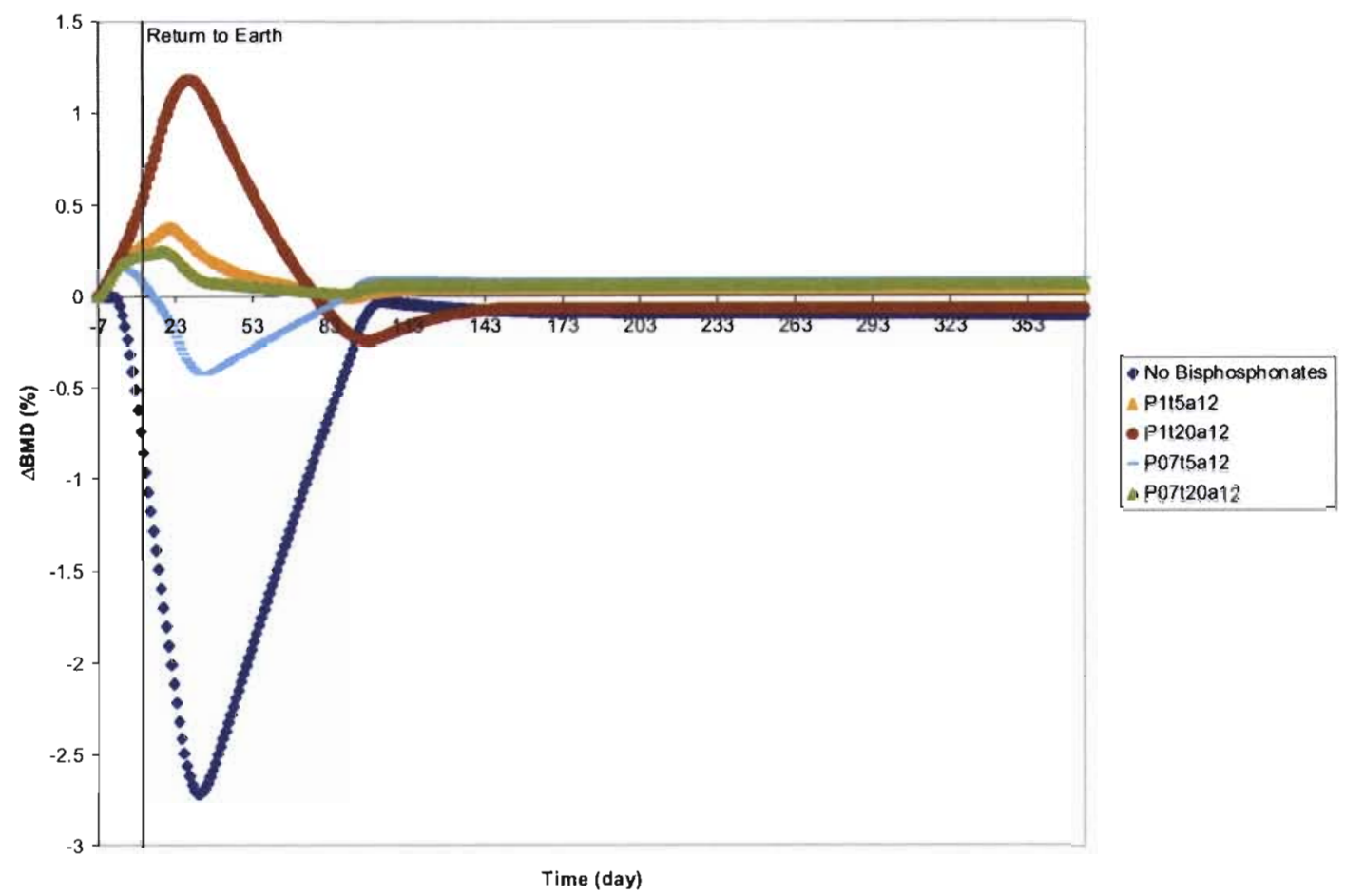

Figure B5. Predicted bisphosphonate effects beginning 7 days preflight on BMD and posttreatment return to Earth from $\mathbf{1 0 - d a y ~ s p a c e f l i g h t . ~}$

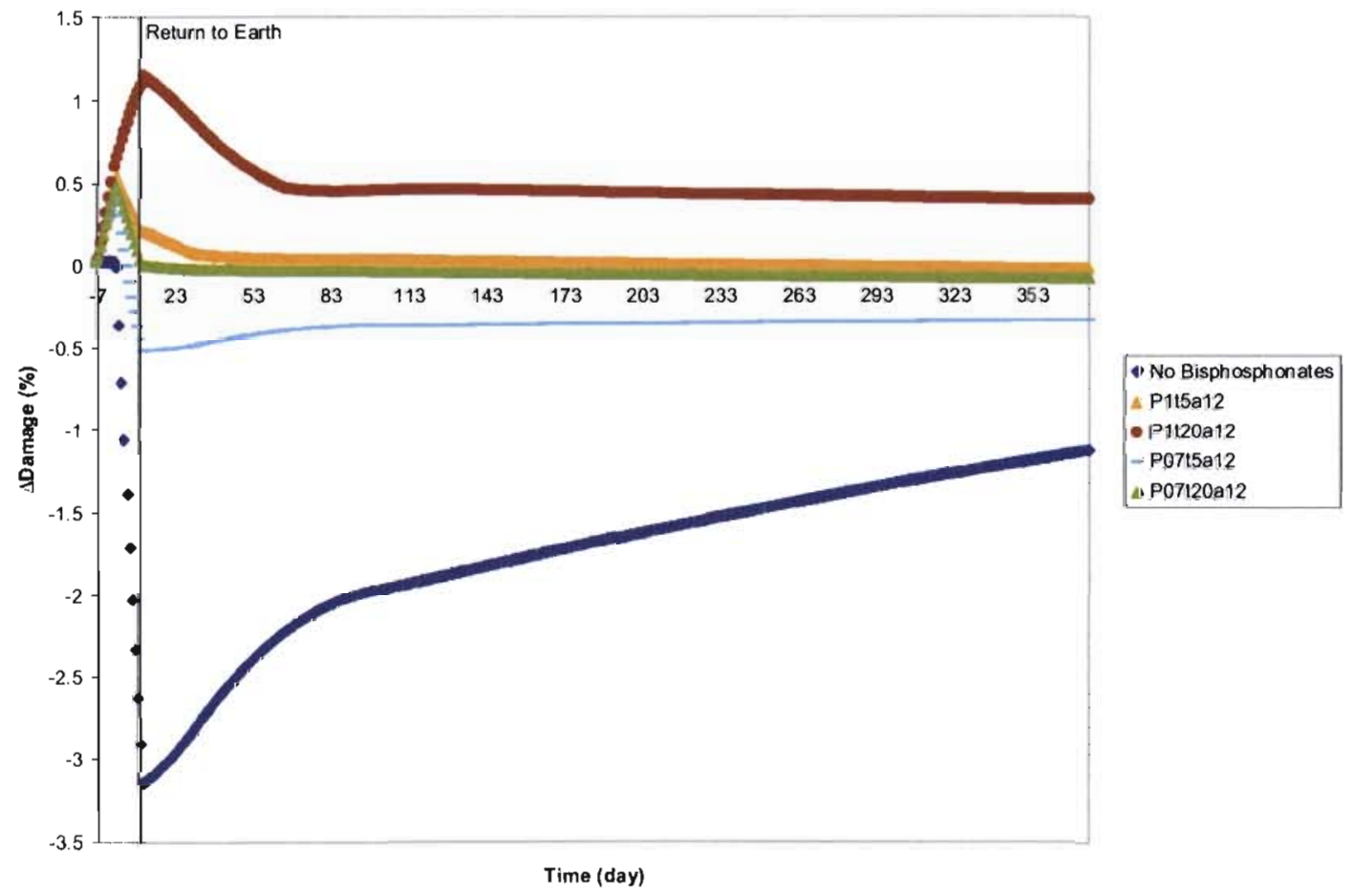

Figure B6. Predicted bisphosphonate effects beginning 7 days preflight on damage accumulation (D) and posttreatment return to Earth from 10-day spaceflight. 


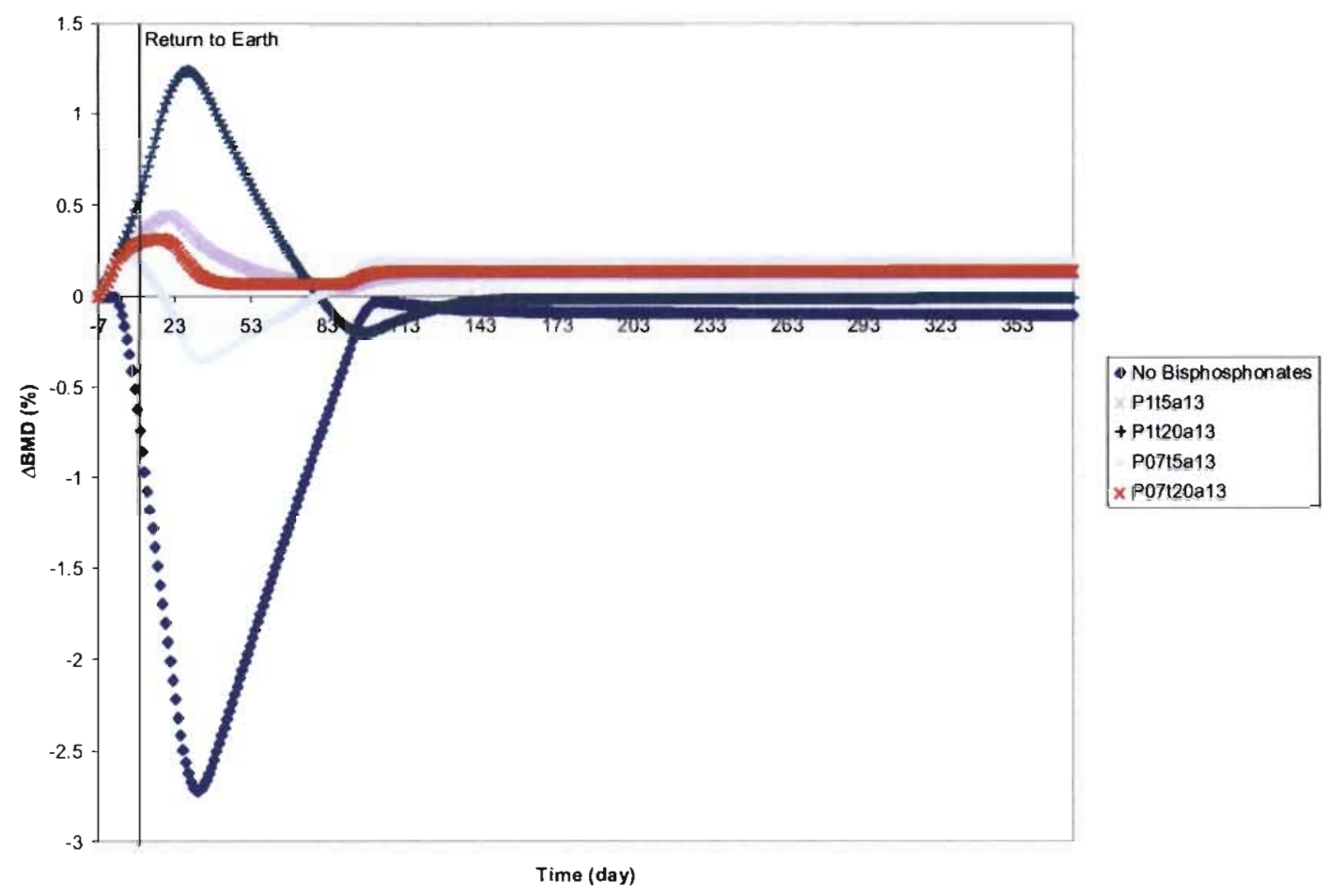

Figure B7. Predicted bisphosphonate effects beginning 7 days preflight on BMD and posttreatment return to Earth from 10-day spaceflight.

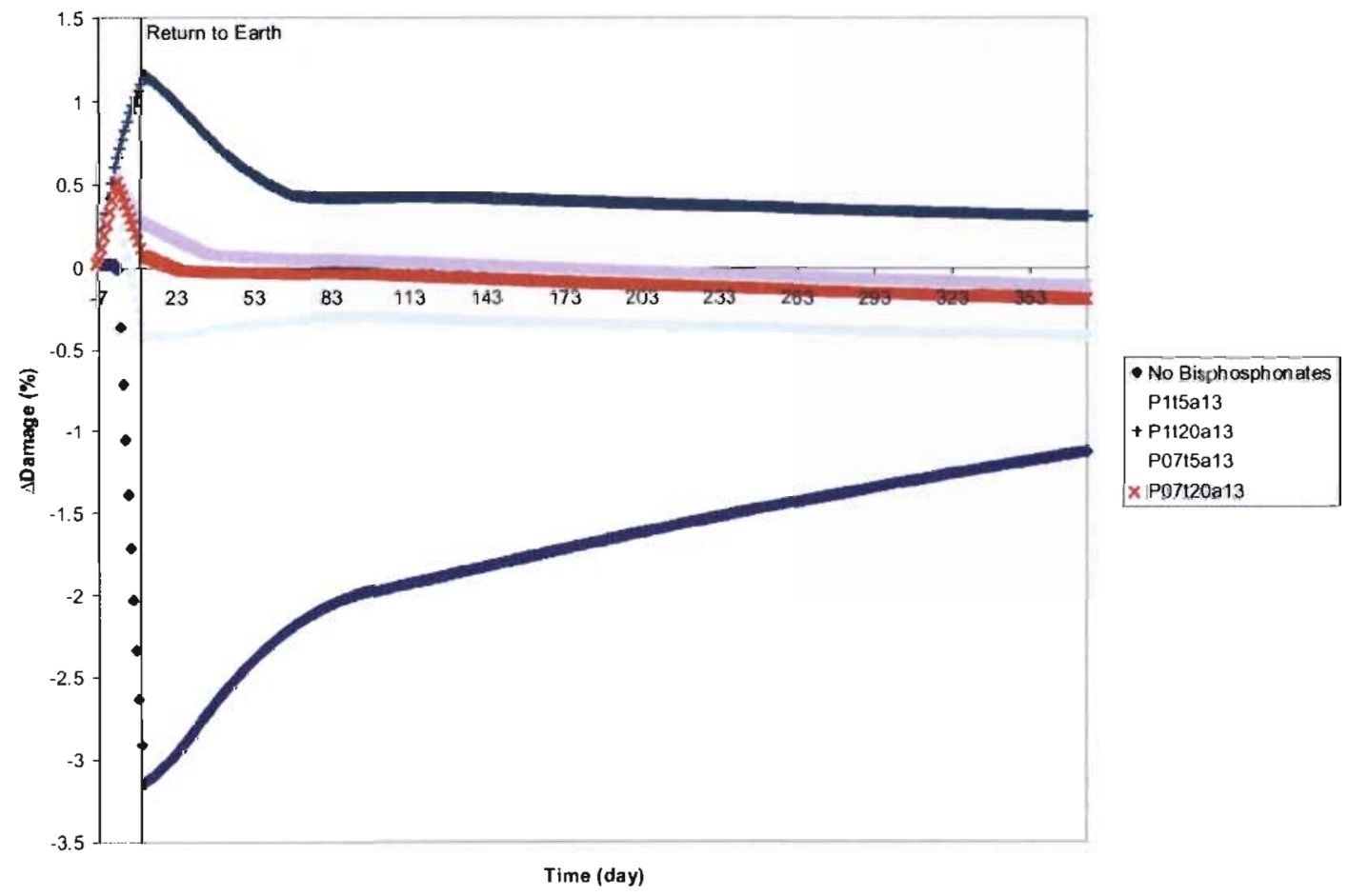

Figure B8. Predicted bisphosphonate effects beginning 7 days preflight on damage accumulation (D) and posttreatment return to Earth from 10-day spaceflight. 


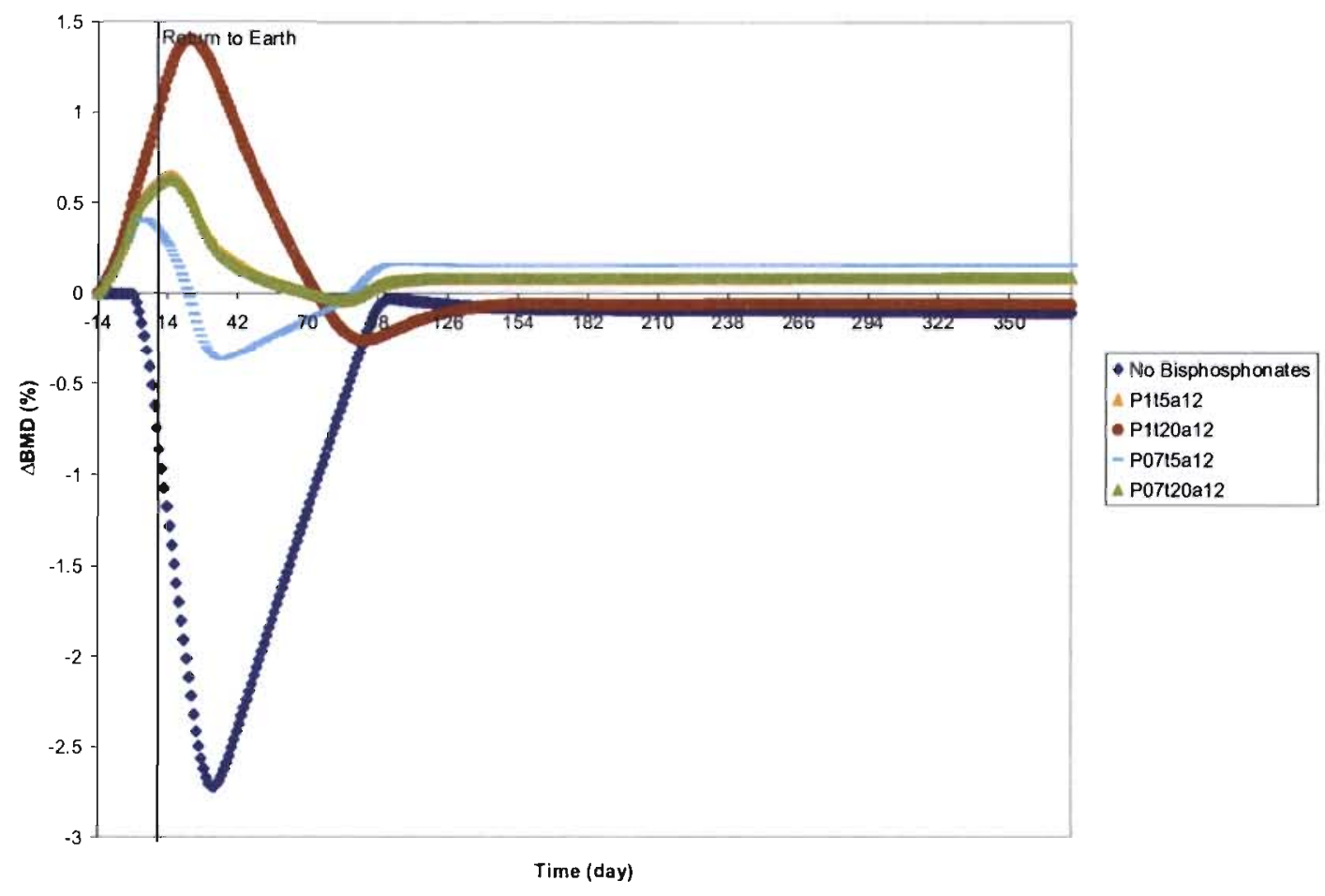

Figure B9. Predicted bisphosphonate effects beginning 14 days preflight on BMD and posttreatment return to Earth from $\mathbf{1 0 - d a y}$ spaceflight.

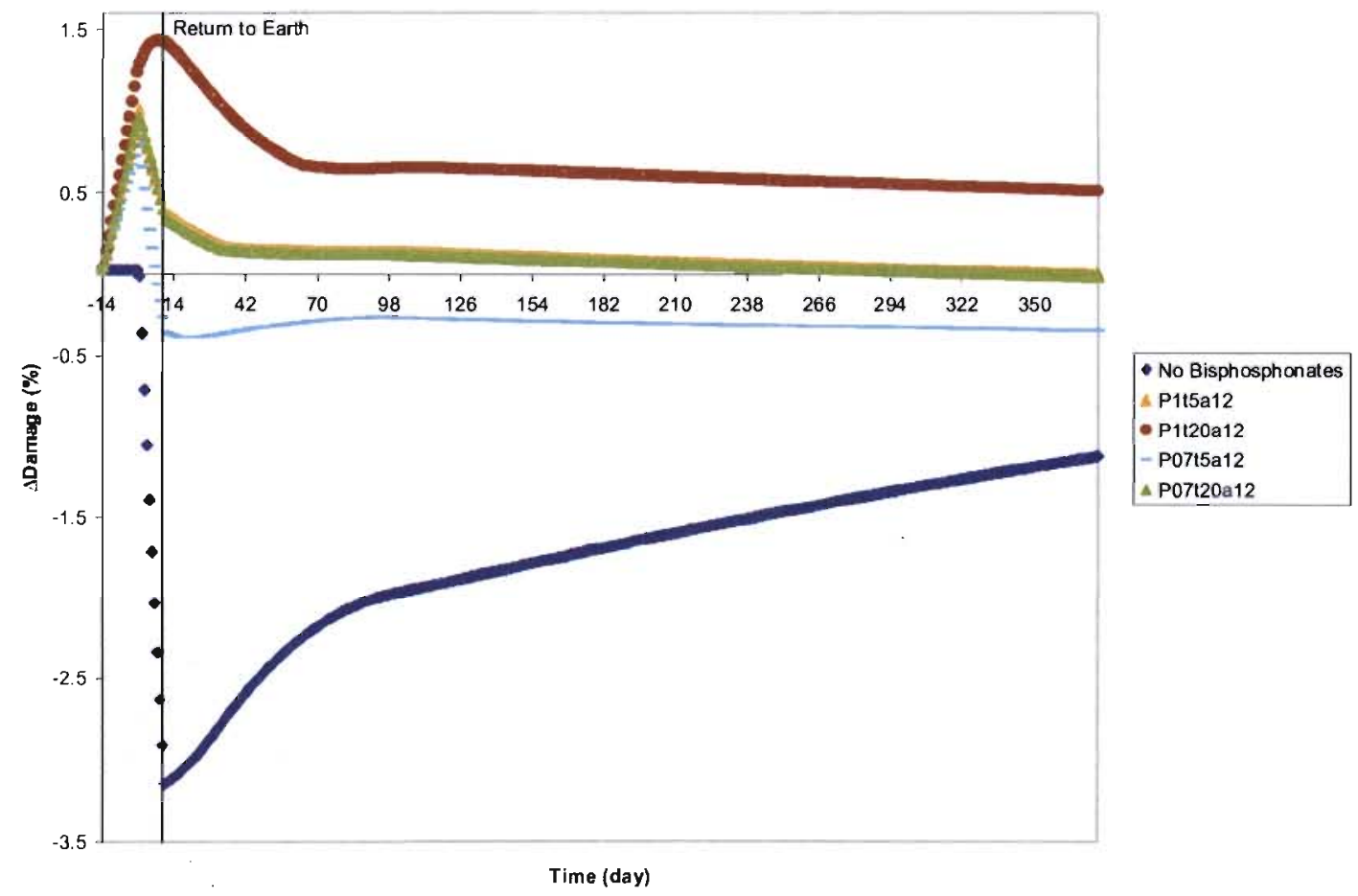

Figure B10. Predicted bisphosphonate effects beginning 14 days preflight on damage accumulation (D) and posttreatment return to Earth from 10-day spaceflight. 


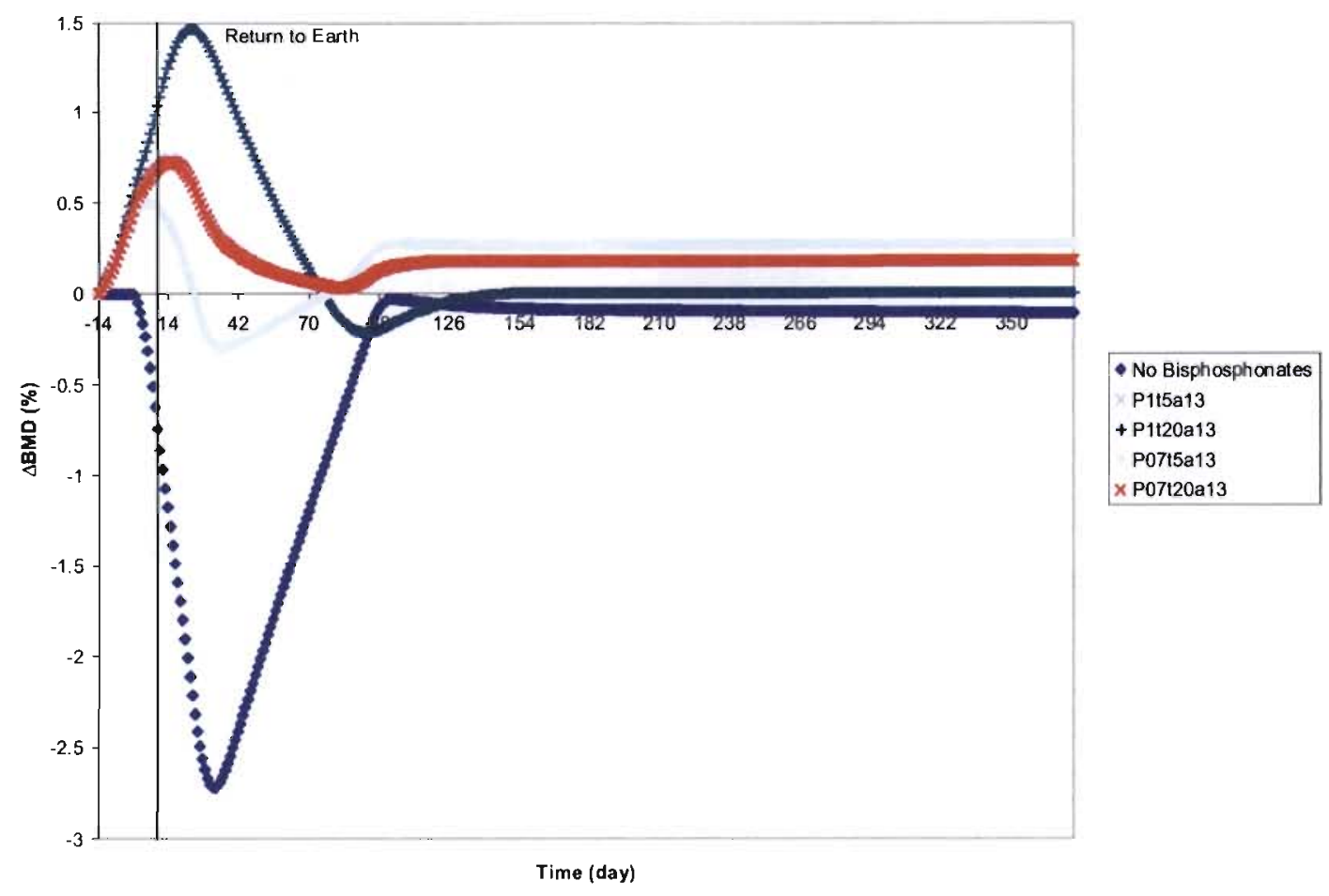

Figure B11. Predicted bisphosphonate effects beginning 14 days preflight on BMD and posttreatment return to Earth from 10-day spaceflight.

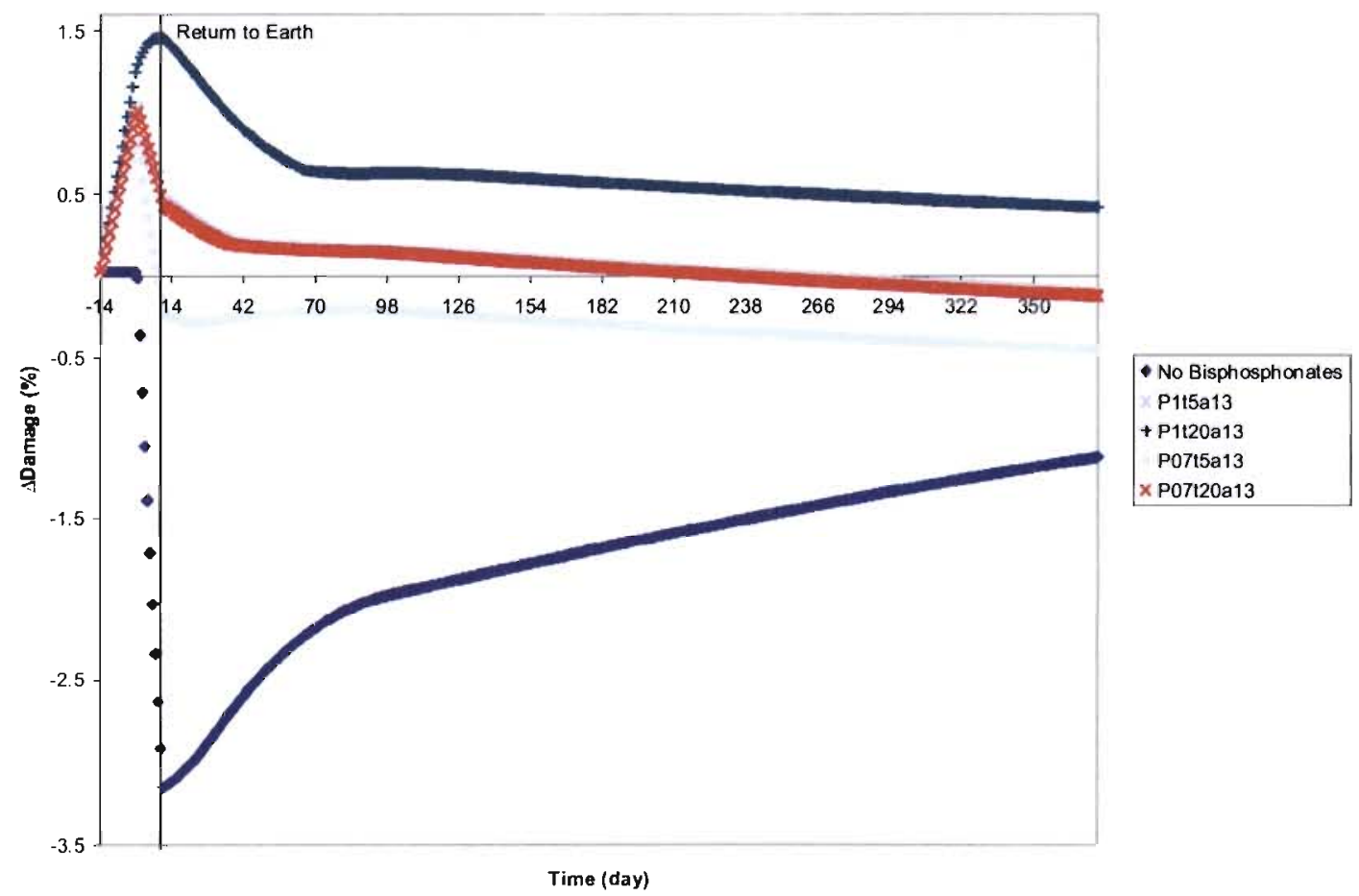

Figure B 12. Predicted bisphosphonate effects beginning 14 days preflight on damage accumulation (D) and posttreatment return to Earth from 10-day spaceflight. 


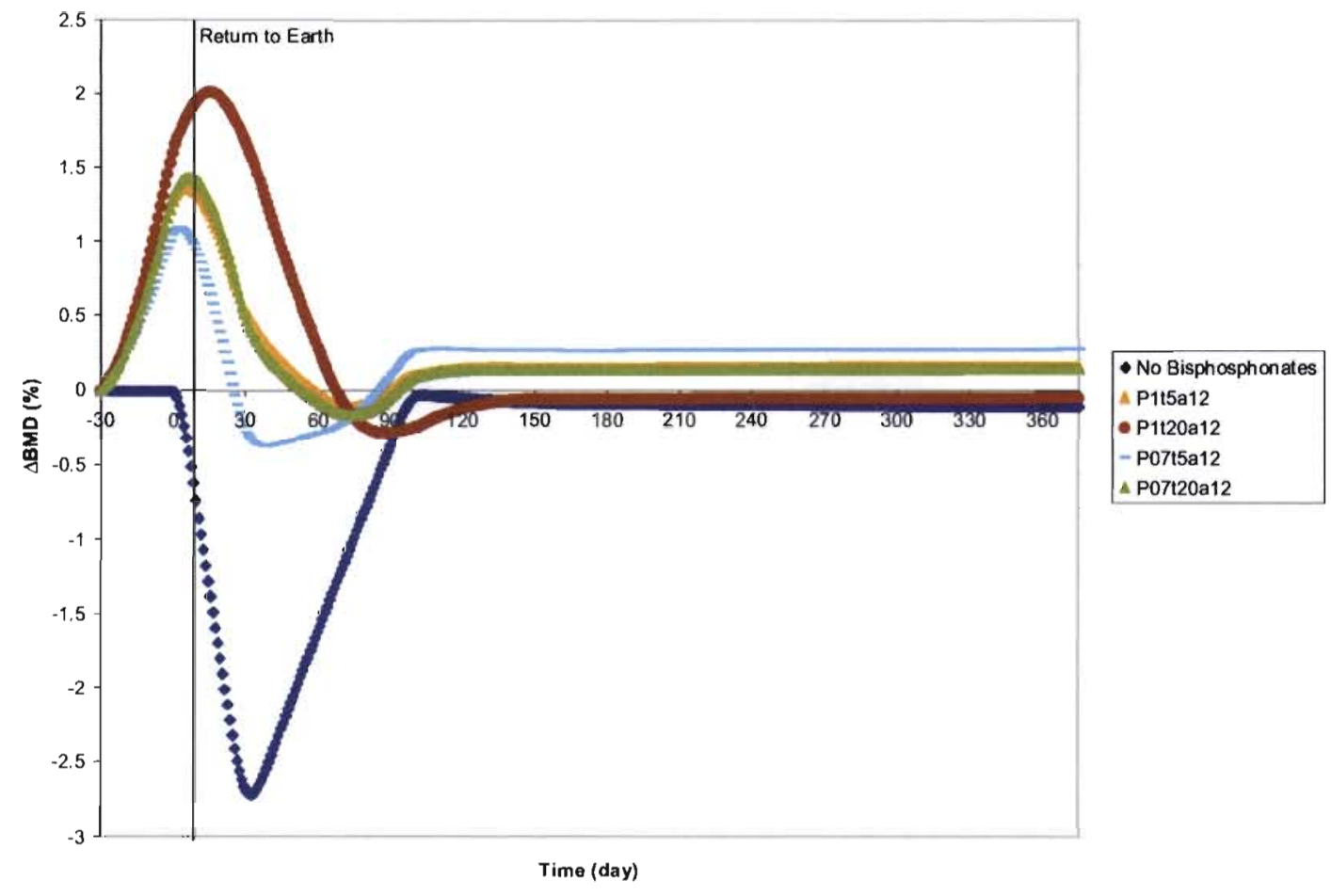

Figure B13. Predicted bisphosphonate effects beginning 30 days preflight on BMD and posttreatment return to Earth from 10-day spaceflight.

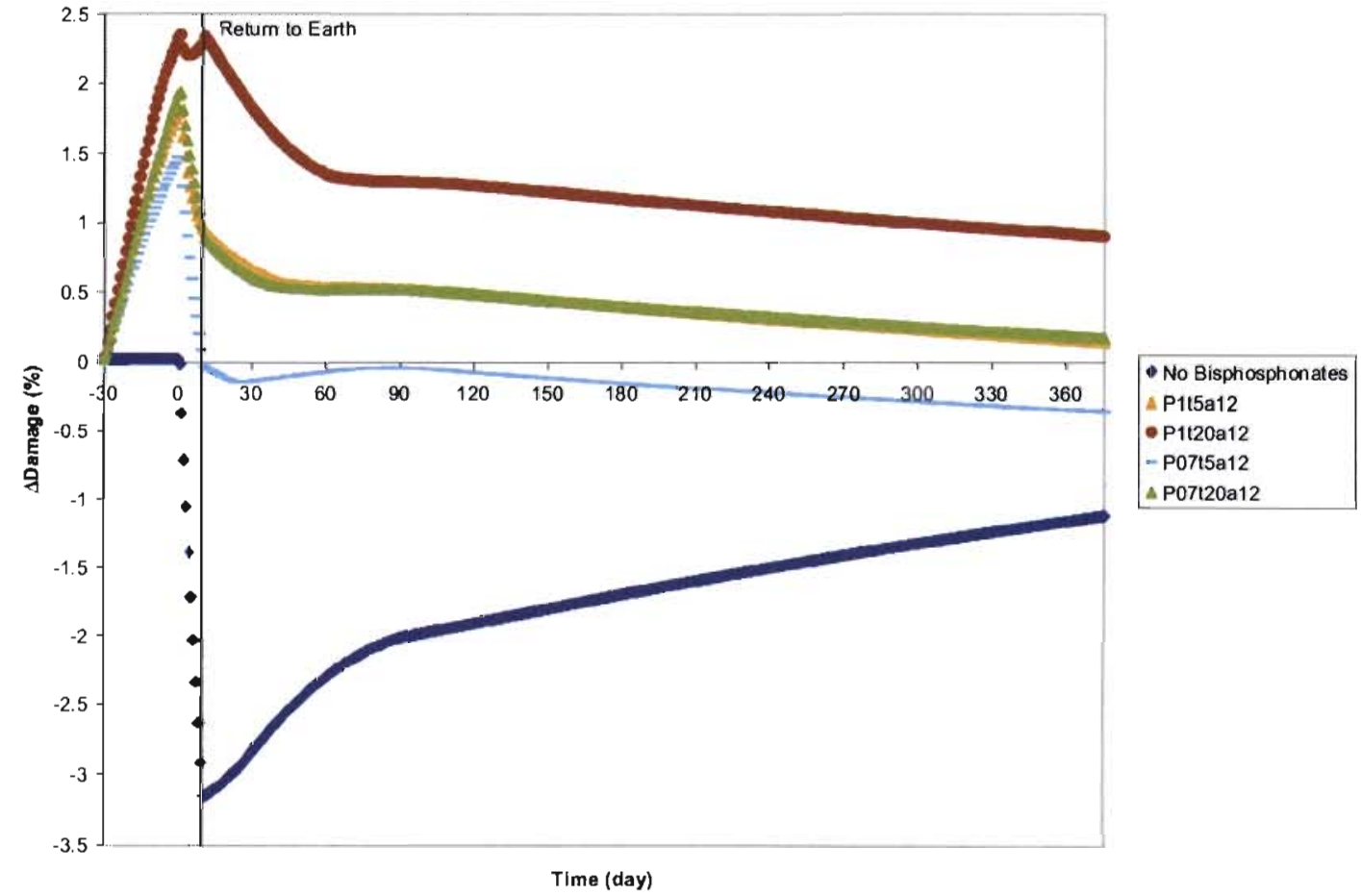

Figure B14. Predicted bisphosphonate effects beginning 30 days preflight on damage accumulation (D) and posttreatment return to Earth from 10-day spaceflight. 


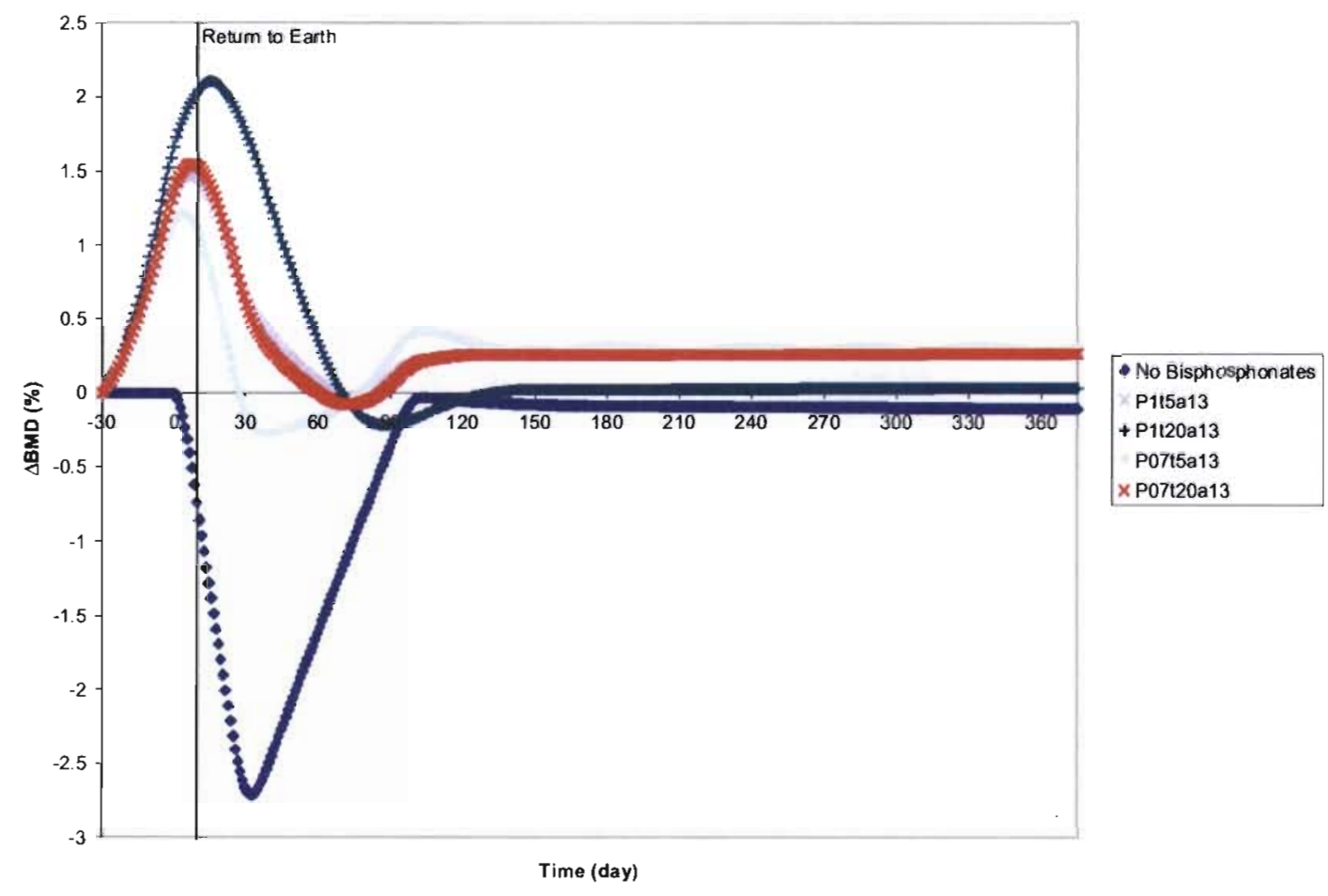

Figure B15. Predicted bisphosphonate effects beginning 30 days preflight on BMD and posttreatment return to Earth from 10-day spaceflight.

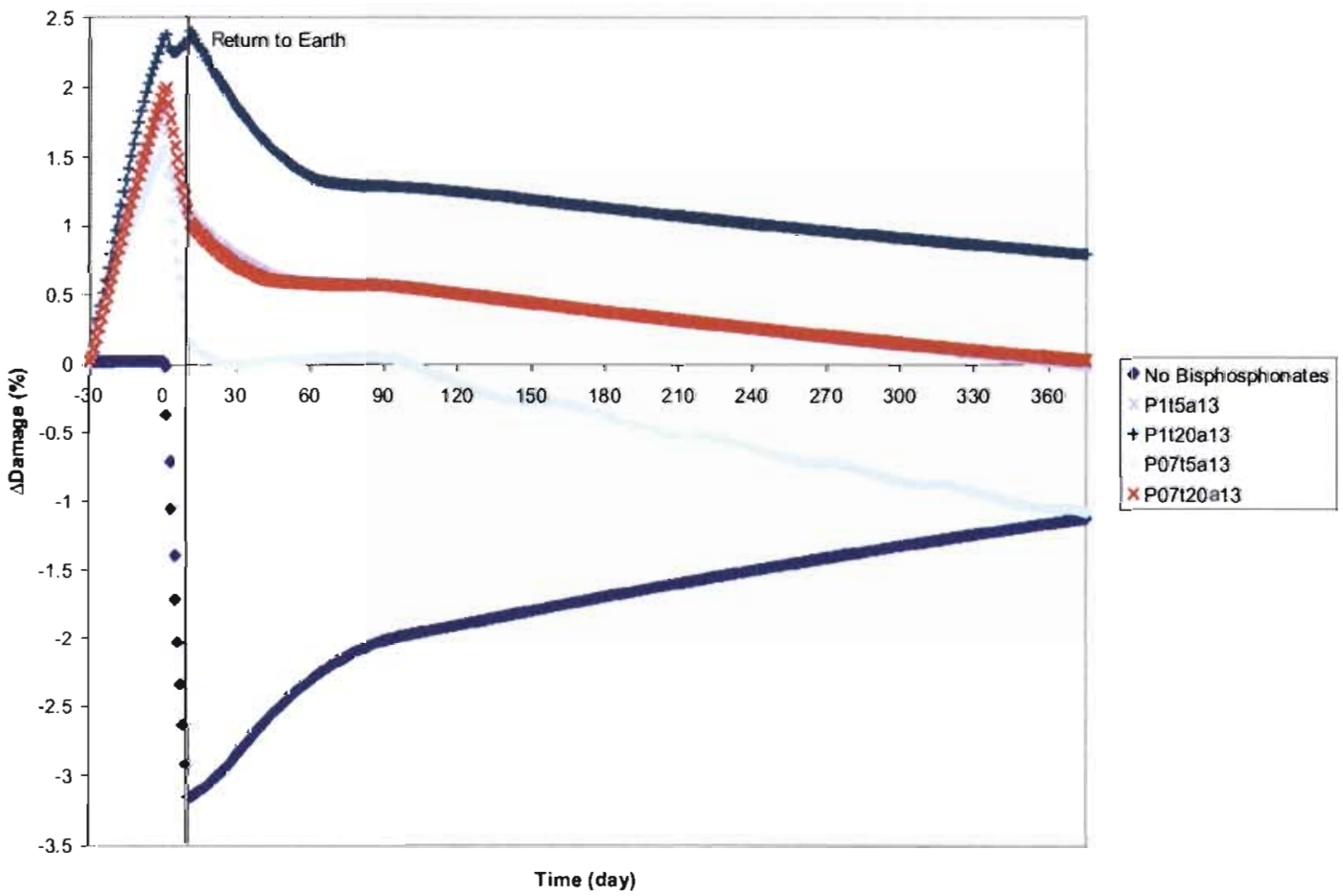

Figure B16. Predicted bisphosphonate effects beginning 30 days preflight on damage accumulation (D) and posttreatment return to Earth from 10-day spaceflight. 


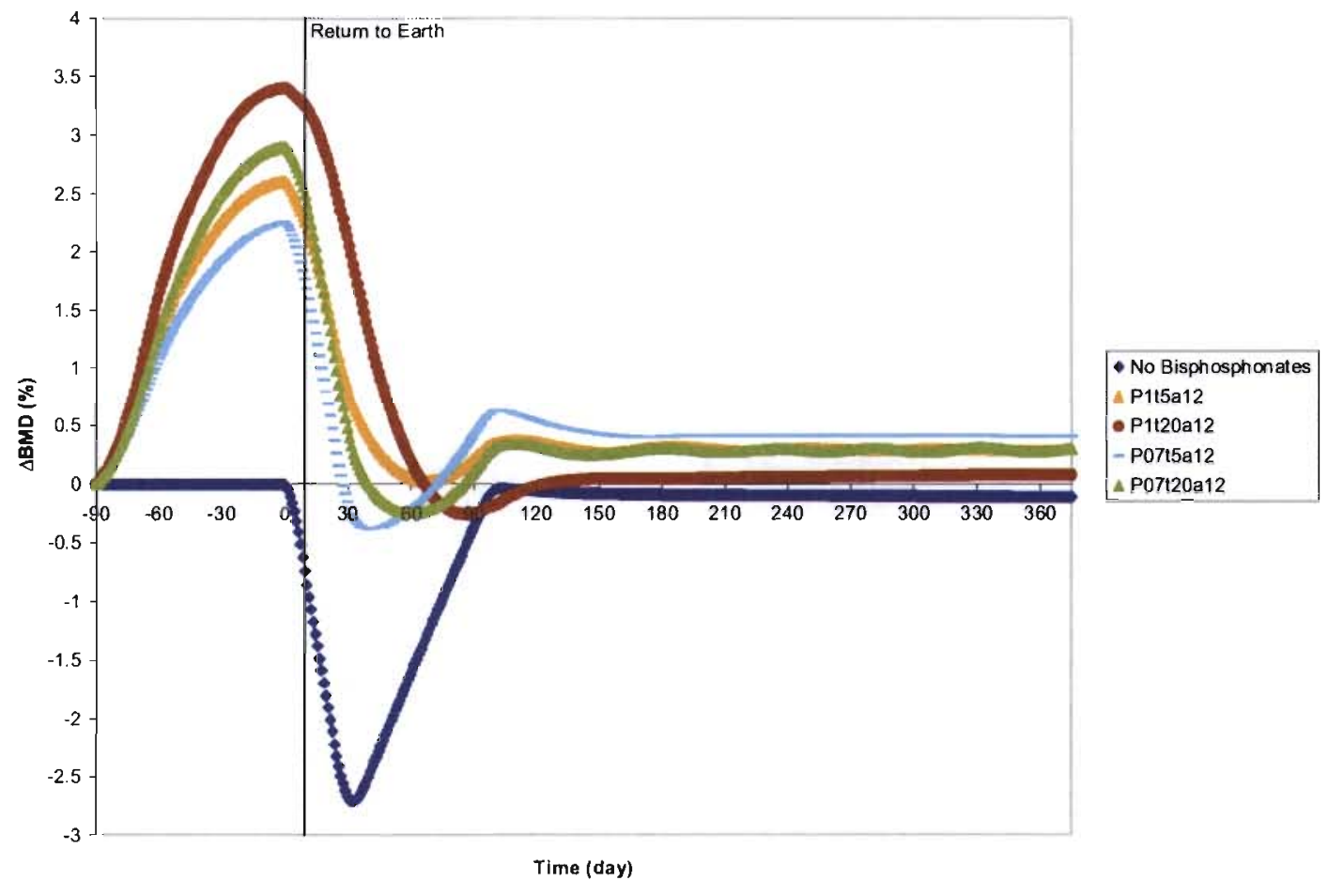

Figure B17. Predicted bisphosphonate effects beginning 90 days preflight on BMD and posttreatment return to Earth from 10-day spaceflight.

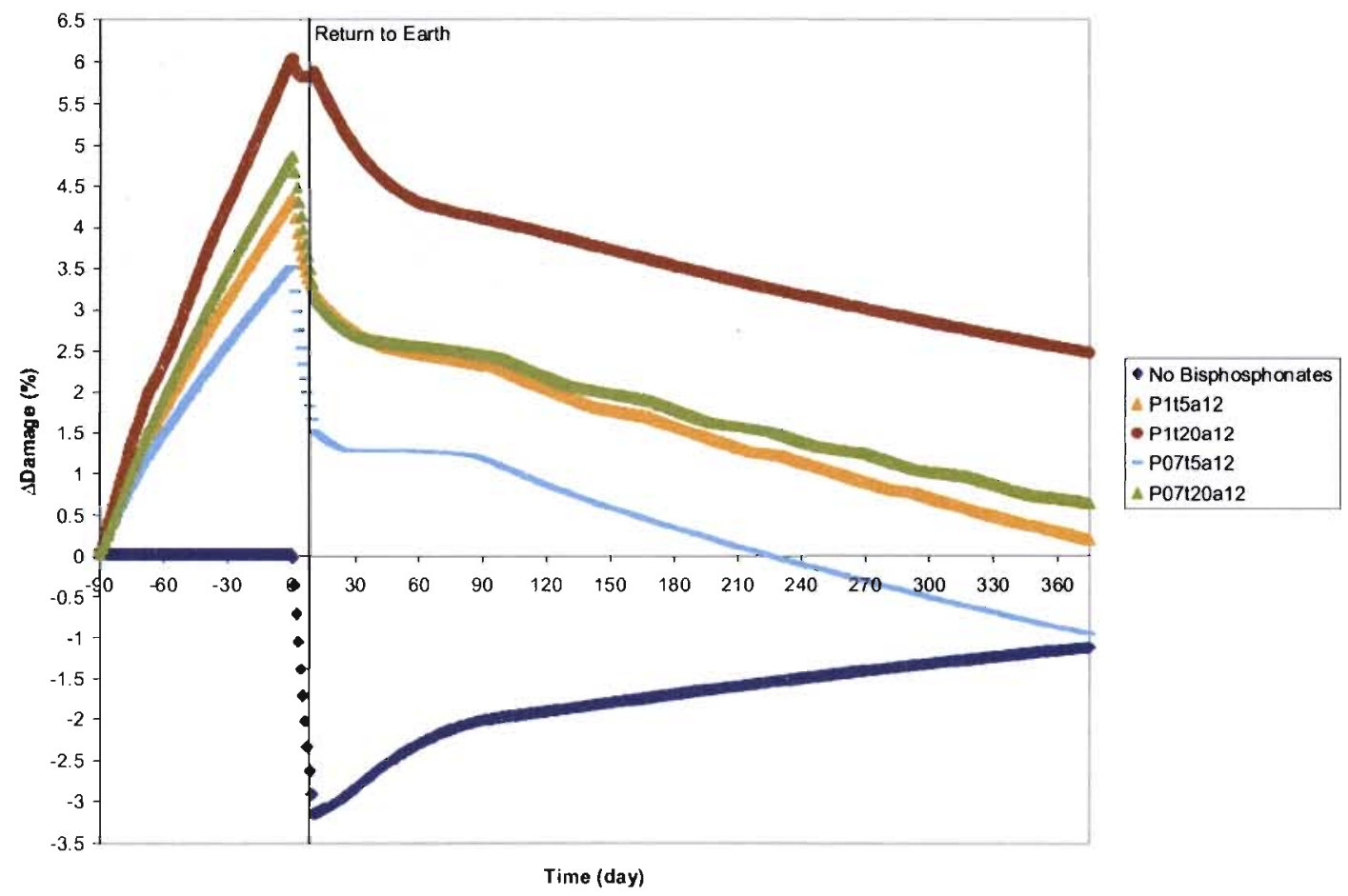

Figure B18. Predicted bisphosphonate effects beginning 90 days preflight on damage accumulation (D) and posttreatment return to Earth from 10-day spaceflight. 


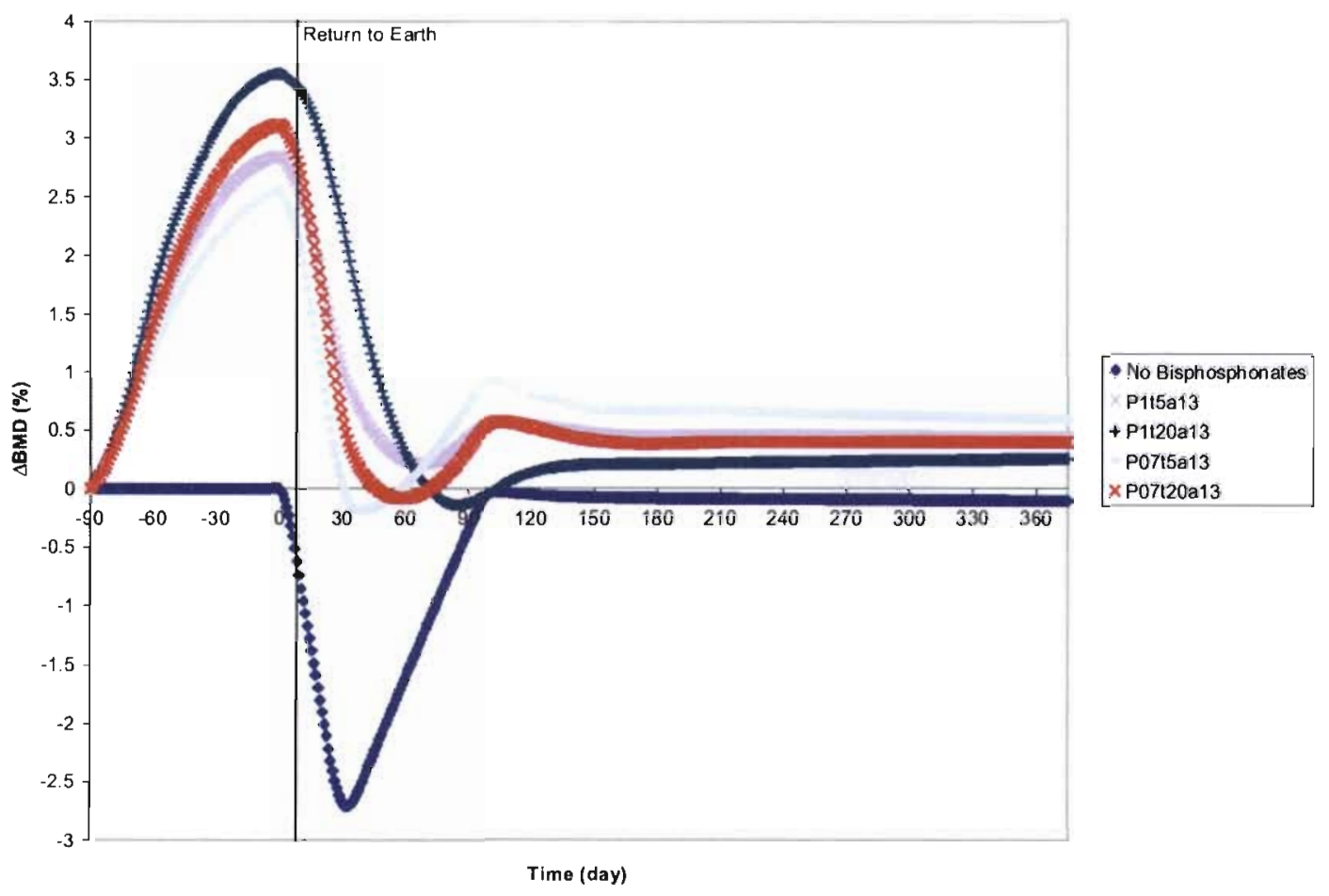

Figure B19. Predicted bisphosphonate effects beginning 90 days preflight on BMD and posttreatment return to Earth from 10-day spaceflight.

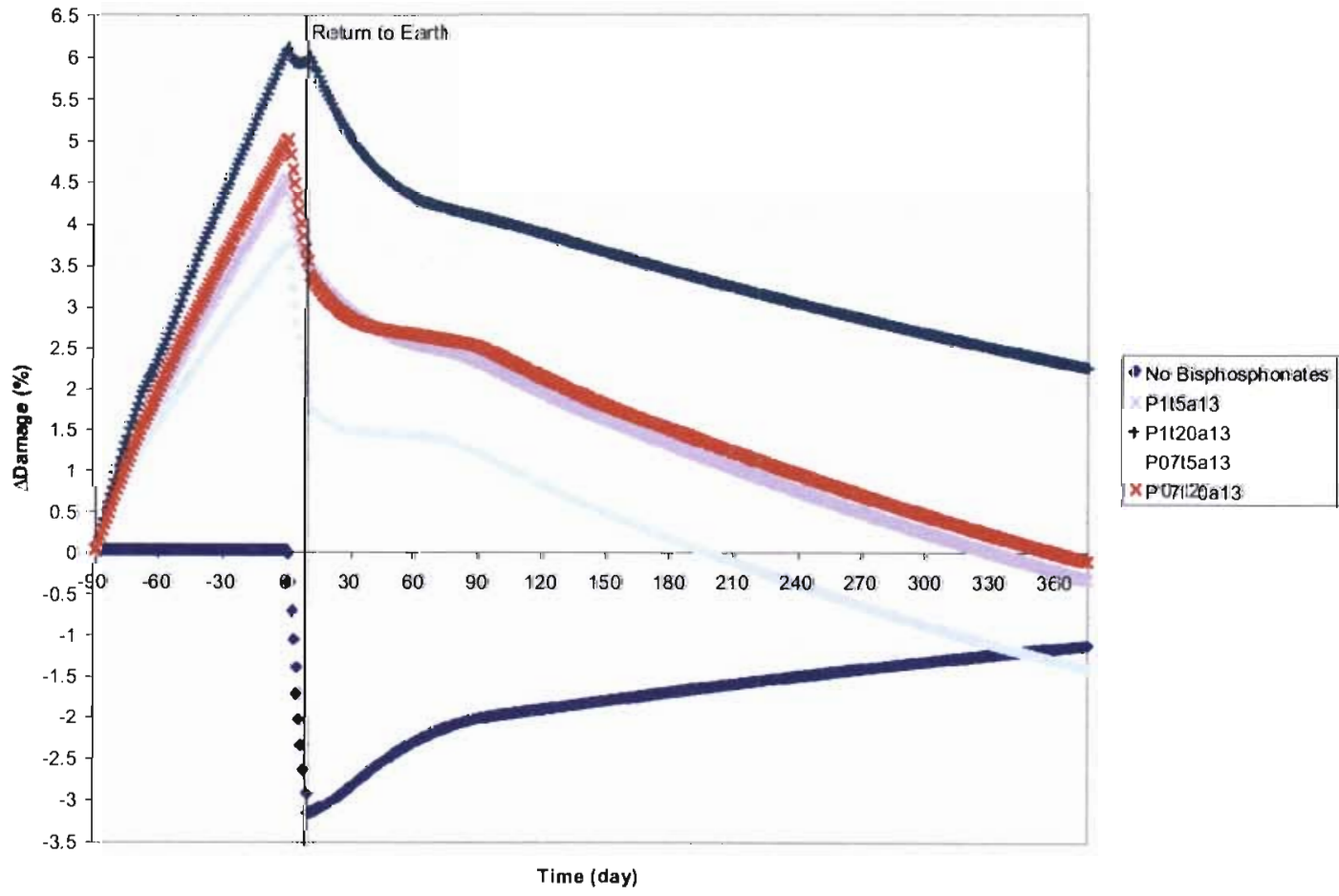

Figure B20. Predicted bisphosphonate effects beginning 90 days preflight on damage accumulation (D) and posttreatment return to Earth from 10-day spaceflight. 


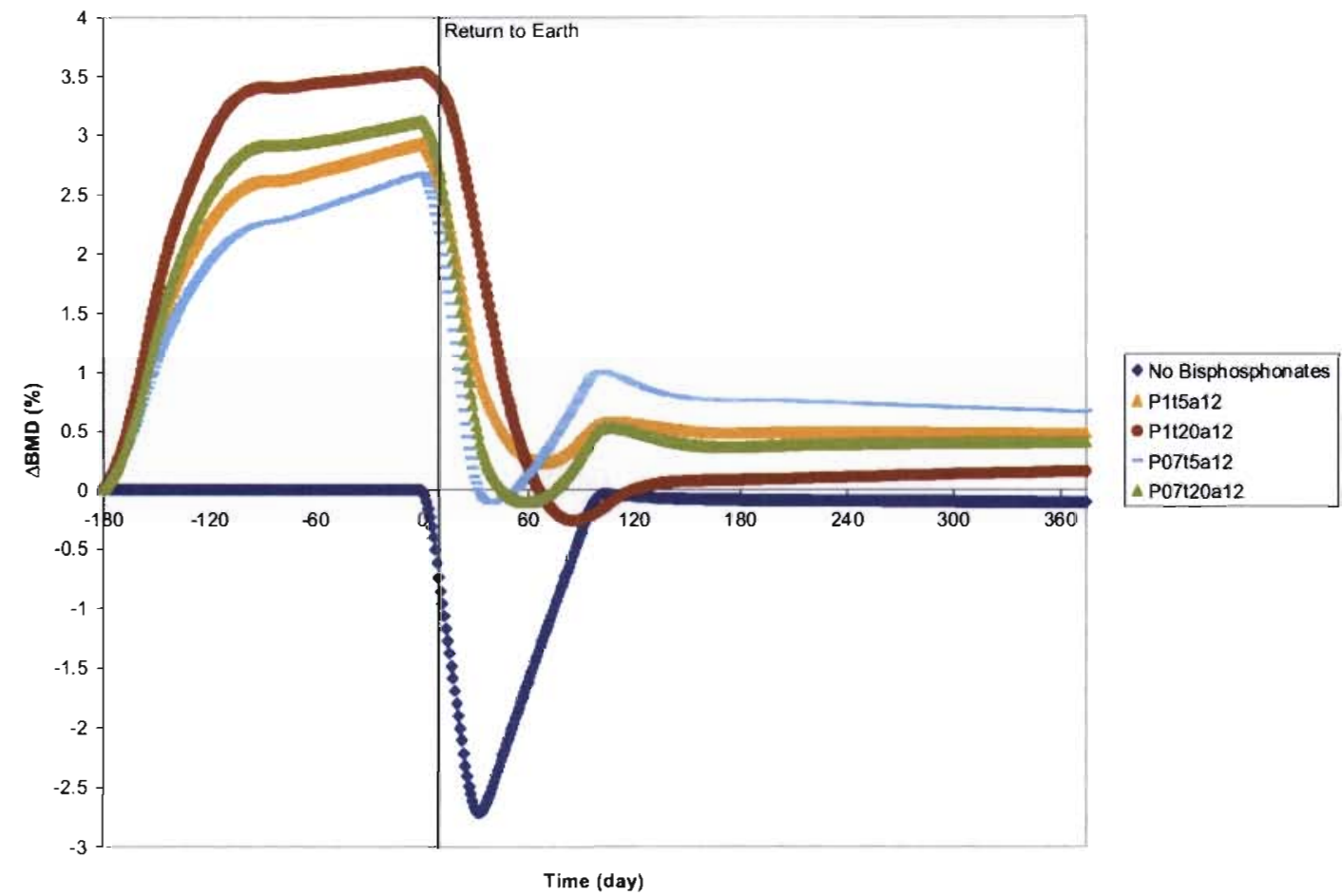

Figure B21. Predicted bisphosphonate effects beginning 180 days preflight on BMD and posttreatment return to Earth from 10-day spaceflight.

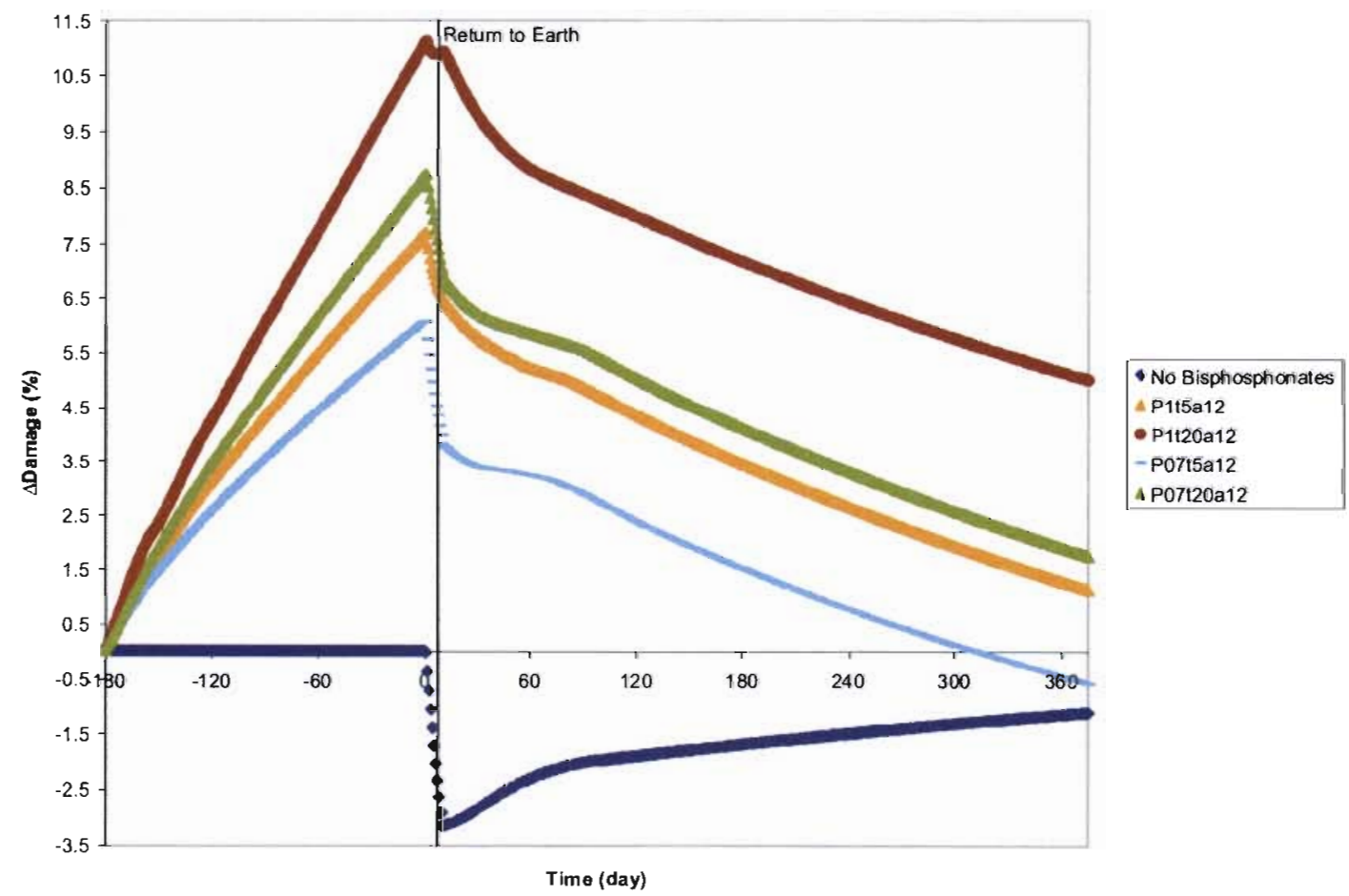

Figure B22. Predicted bisphosphonate effects beginning 180 days preflight on damage accumulation (D) and posttreatment return to Earth from 10-day spaceflight. 


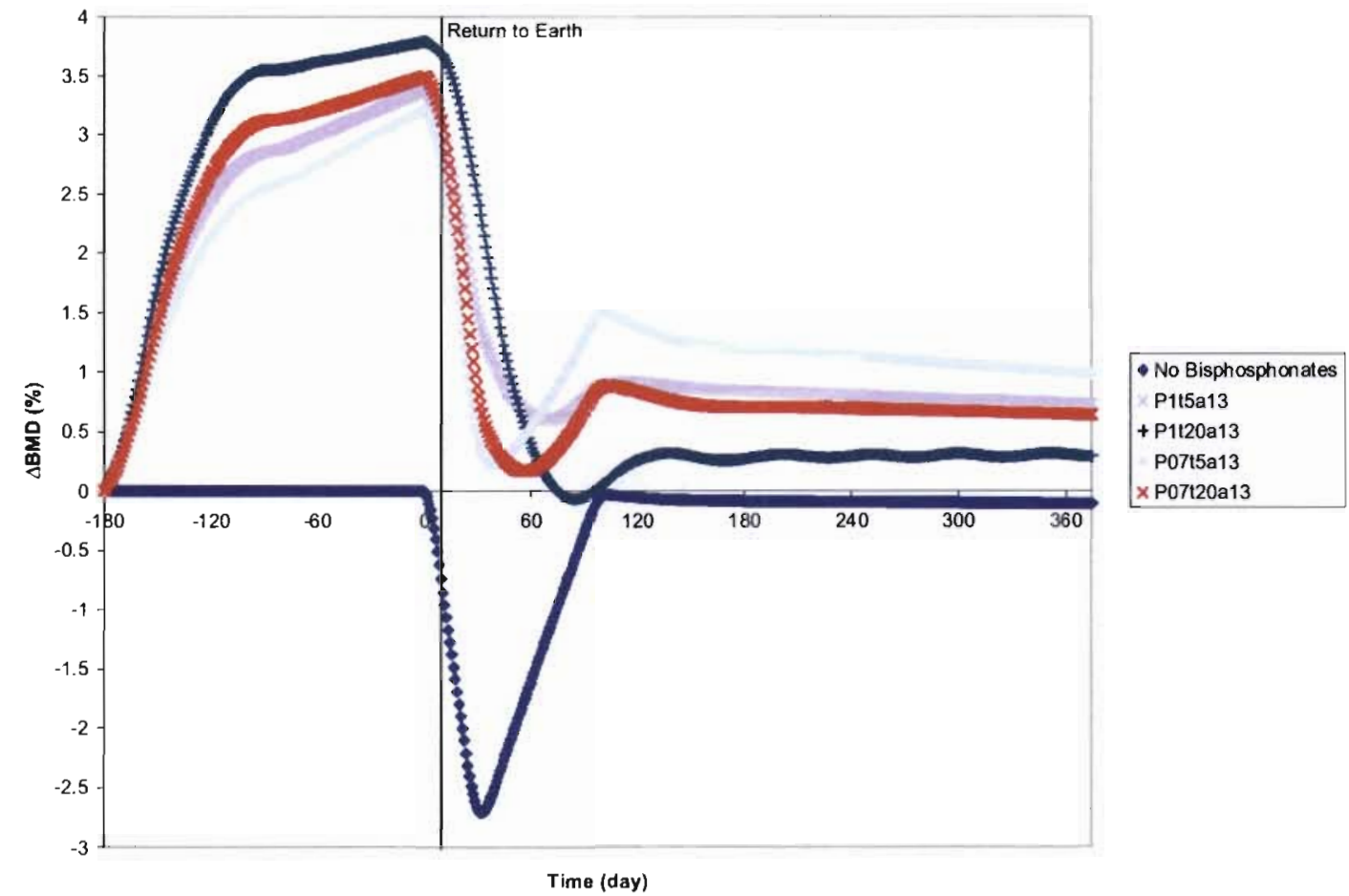

Figure B23. Predicted bisphosphonate effects beginning 180 days preflight on BMD and posttreatment return to Earth from 10-day spaceflight.

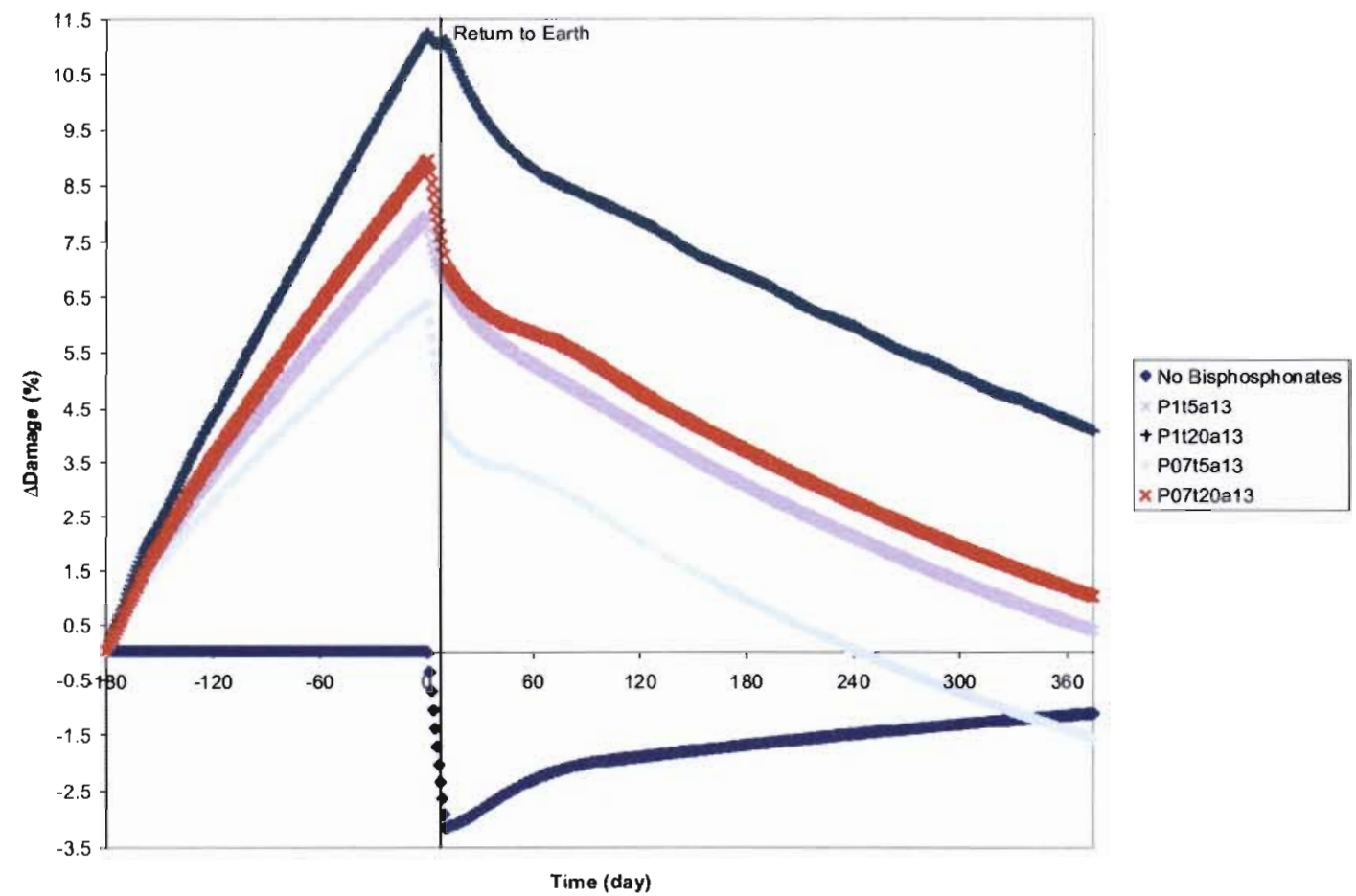

Figure B24. Predicted bisphosphonate effects beginning 180 days preflight on damage accumulation (D) and posttreatment return to Earth from 10-day spaceflight. 


\section{APPENDIX C: FIGURES (90-DAY SPACEFLIGHT)}

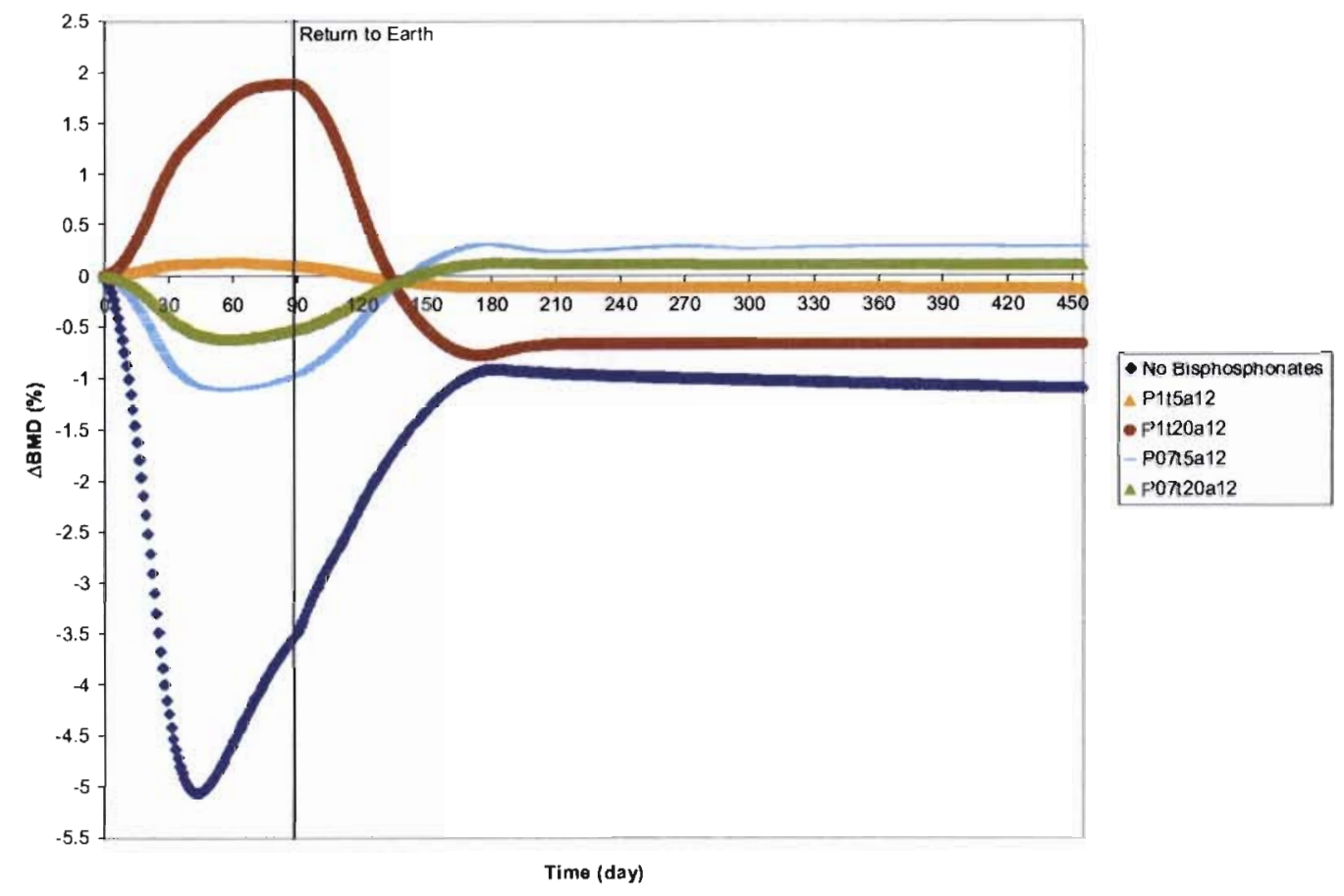

Figure C1. Predicted bisphosphonate effects on BMD and posttreatment return to Earth from 90-day spaceflight.

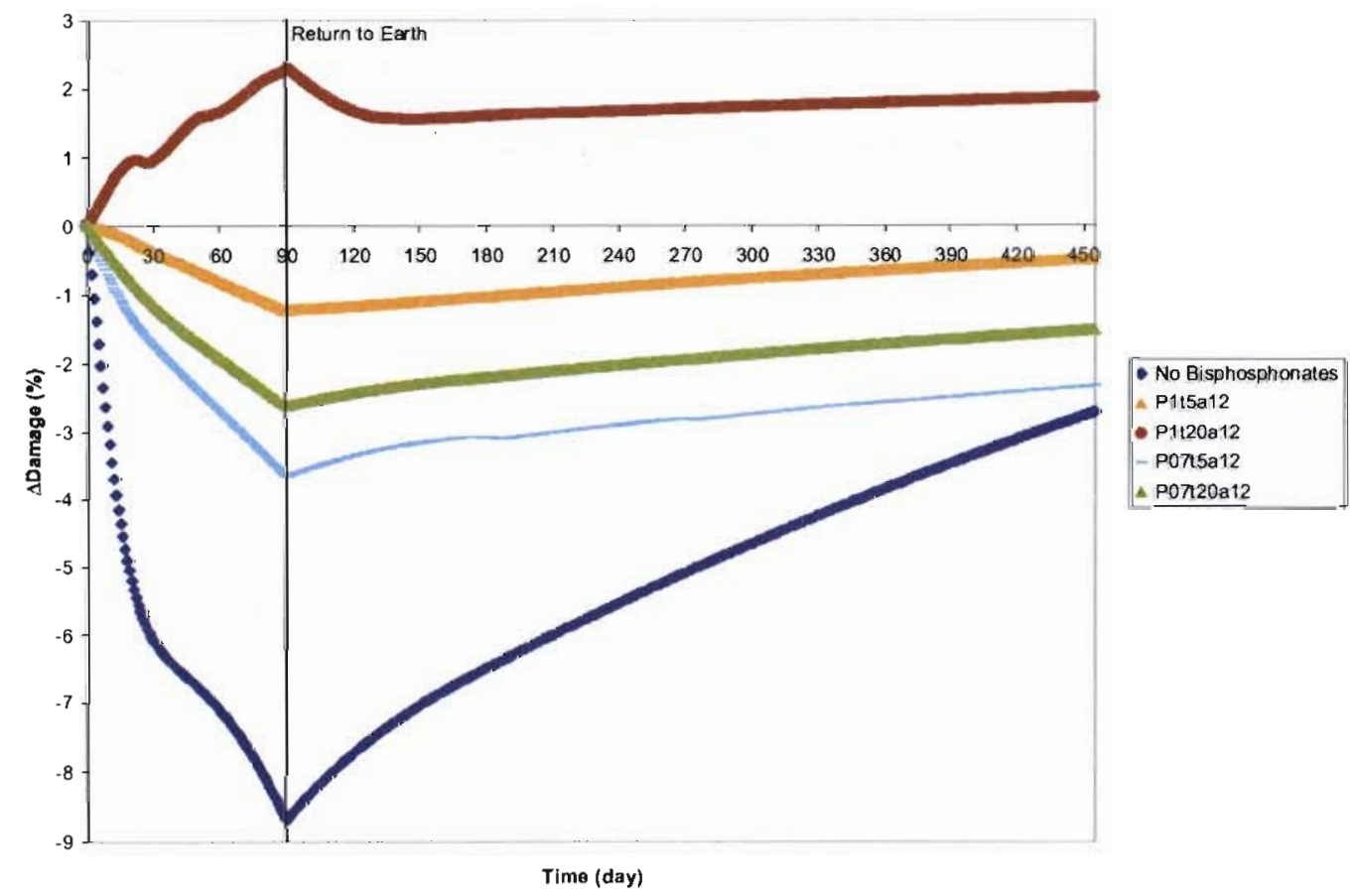

Figure C2. Predicted bisphosphonate effects on damage accumulation (D) and posttreatment return to Earth from 90-day spaceflight. 


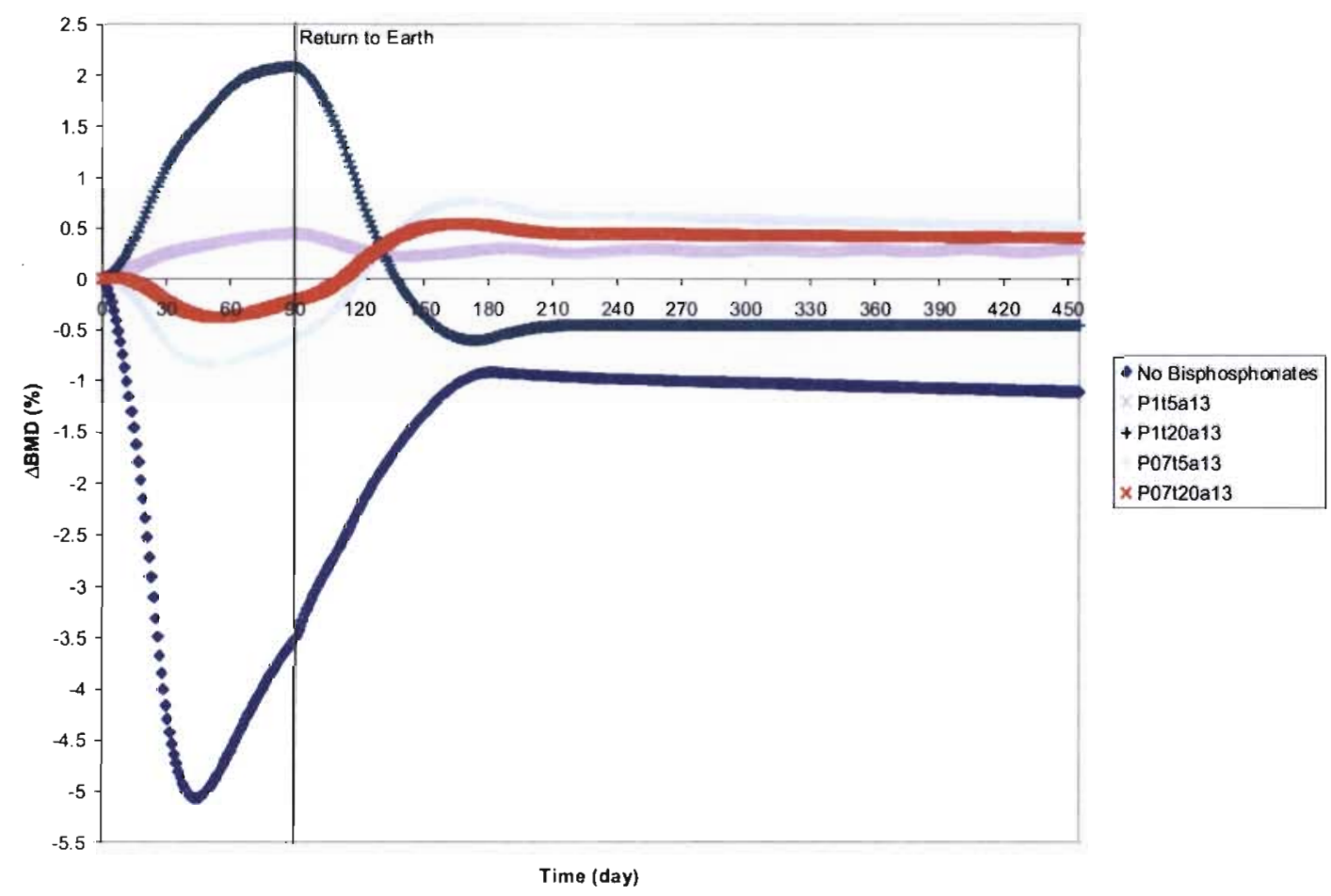

Figure C3. Predicted bisphosphonate effects on BMD and posttreatment return to Earth from 90-day spaceflight.

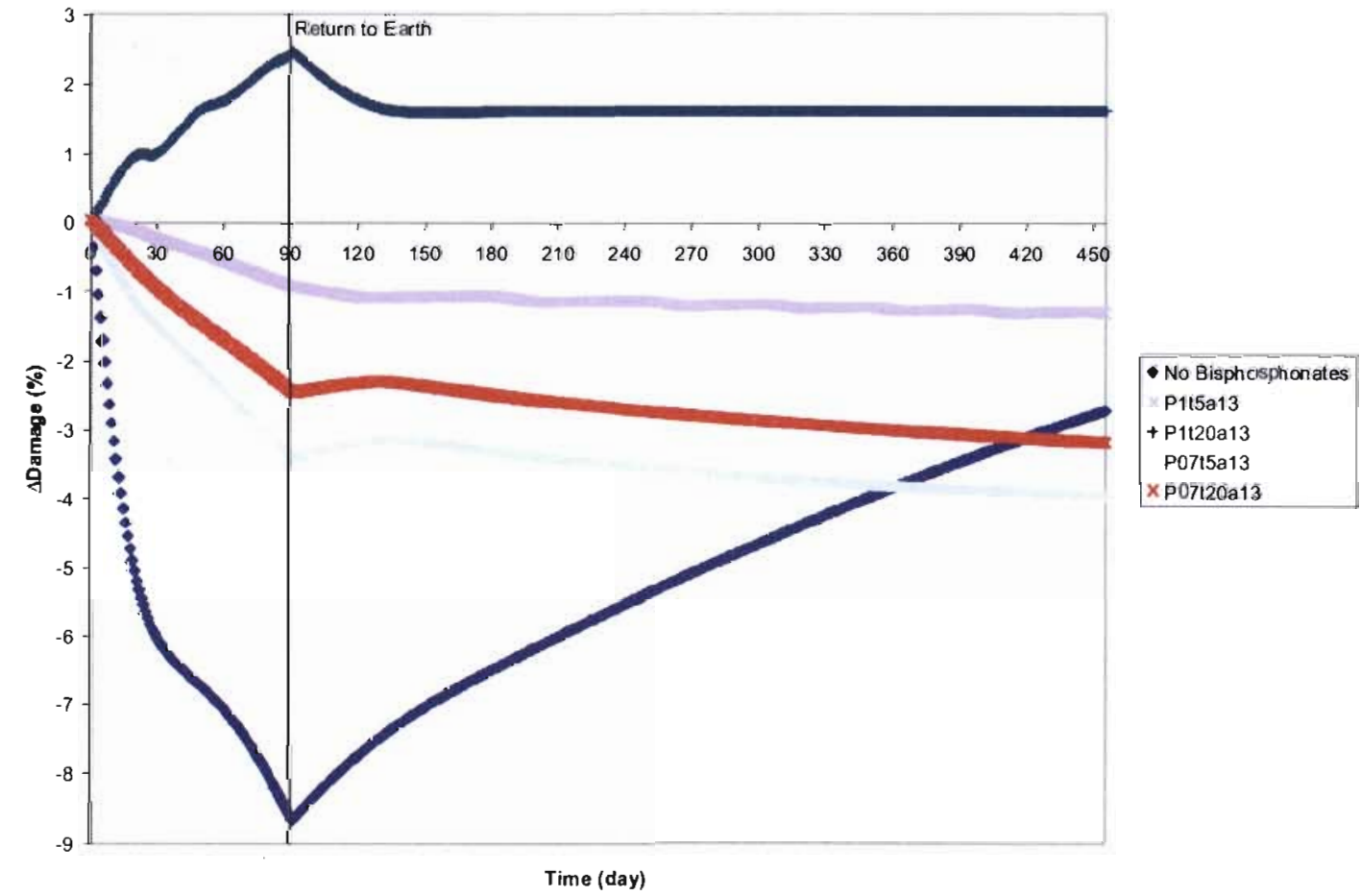

Figure C4. Predicted bisphosphonate effects on damage accumulation (D) and posttreatment return to Earth from 90-day spaceflight. 


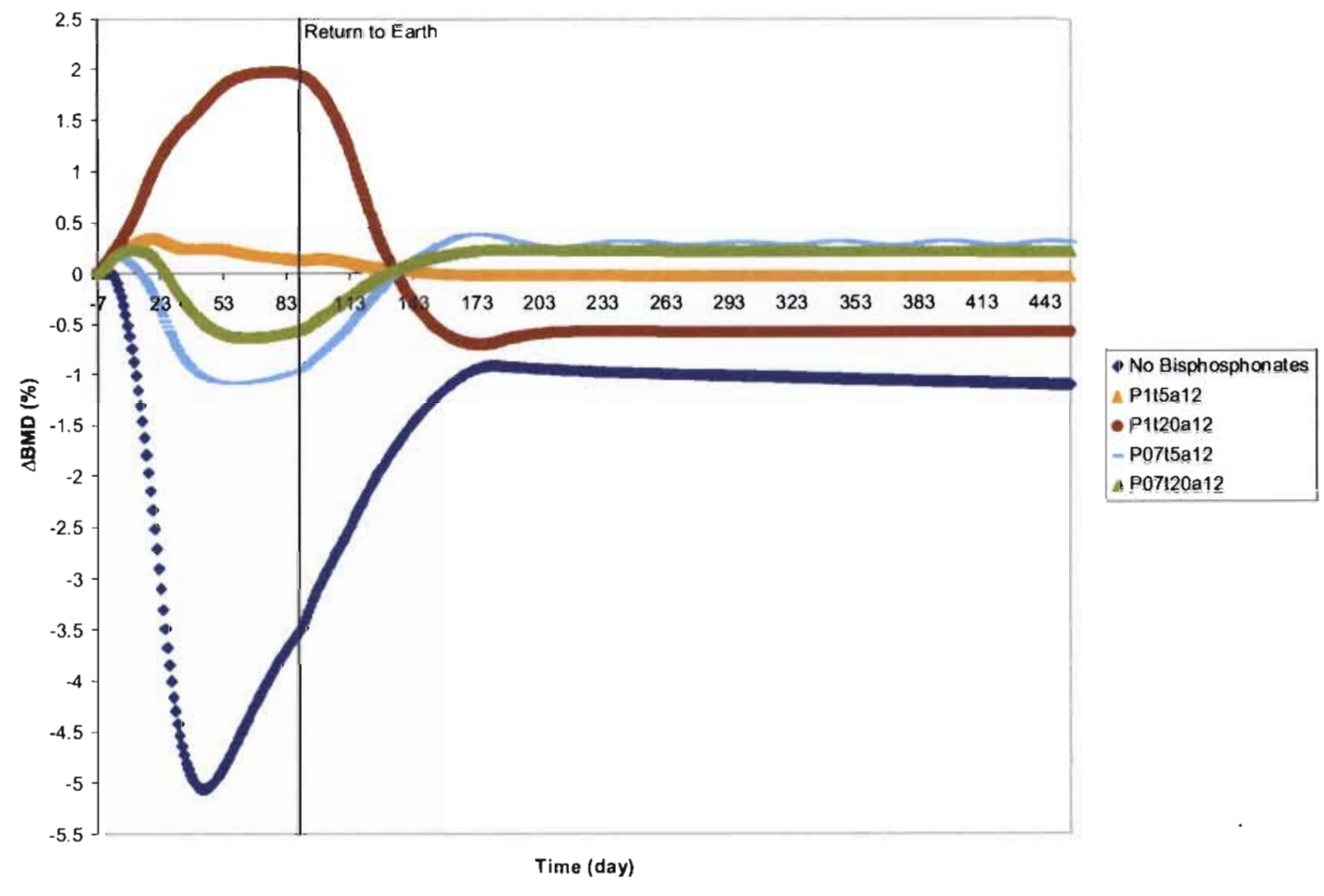

Figure C5. Predicted bisphosphonate effects beginning 7 days preflight on BMD and posttreatment return to Earth from 90 -day spaceflight.

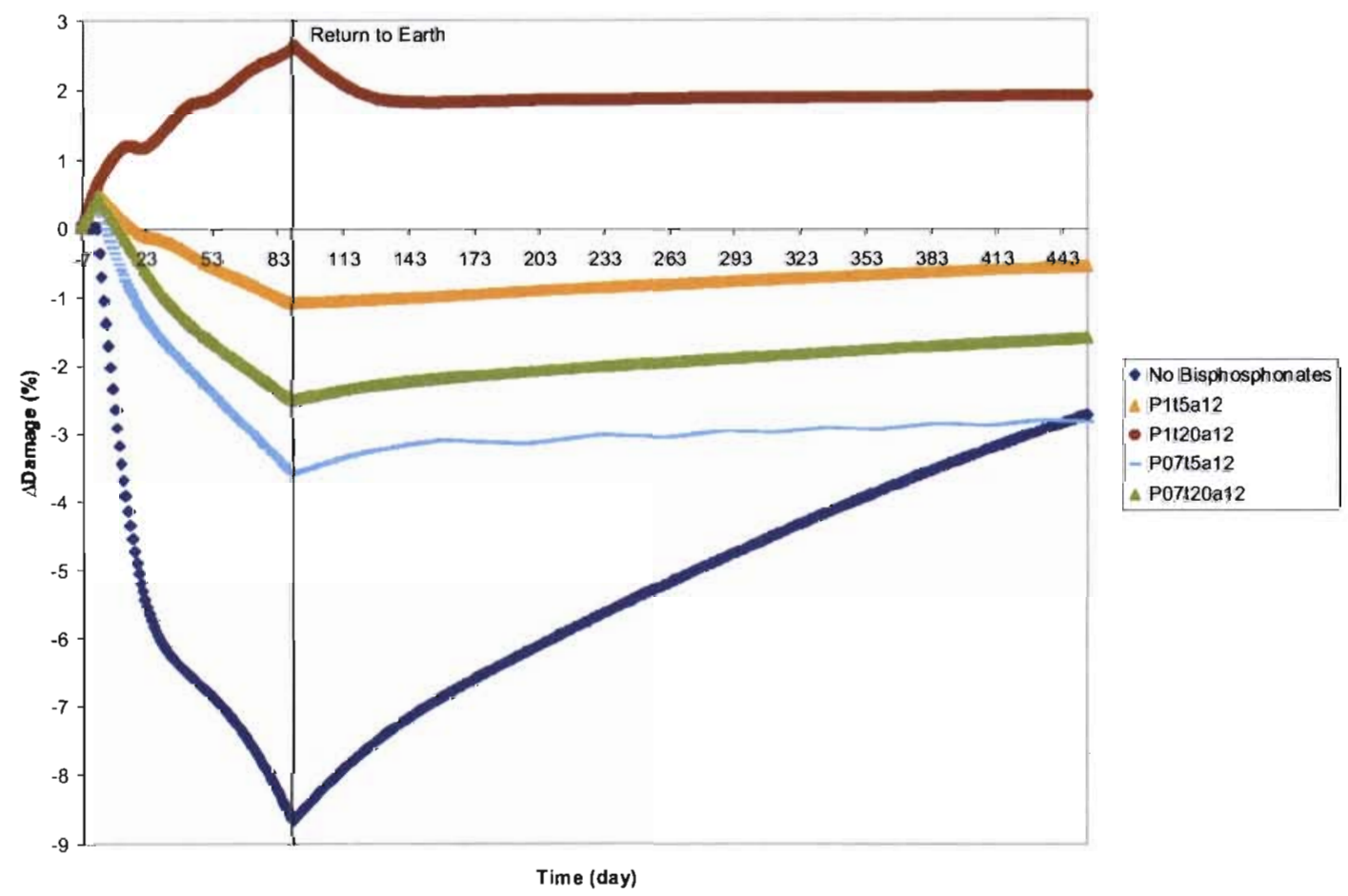

Figure C6. Predicted bisphosphonate effects beginning 7 days preflight on damage accumulation (D) and posttreatment return to Earth from 90-day spaceflight. 


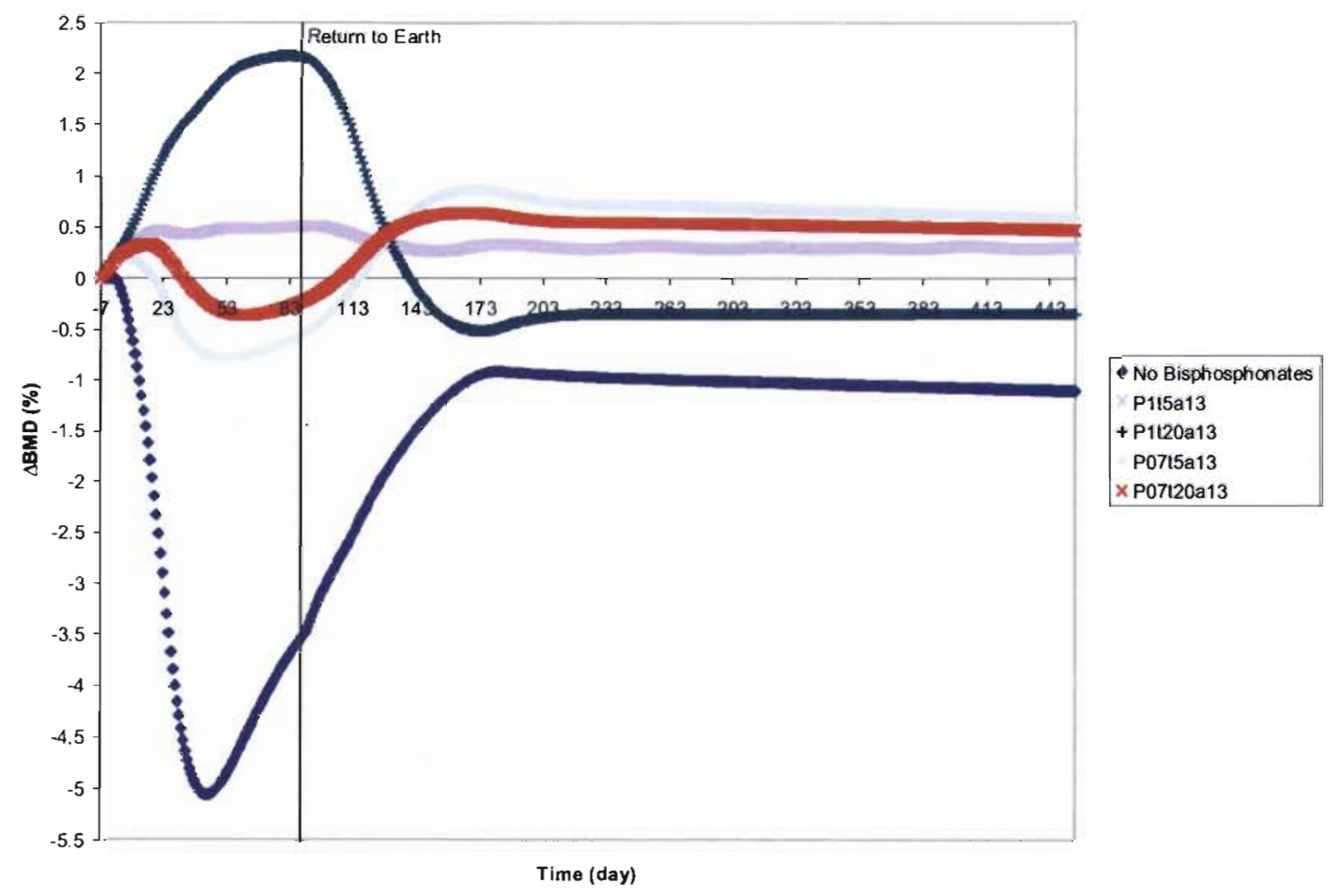

Figure C7. Predicted bisphosphonate effects beginning 7 days preflight on BMD and posttreatment return to Earth from 90 -day spaceflight.

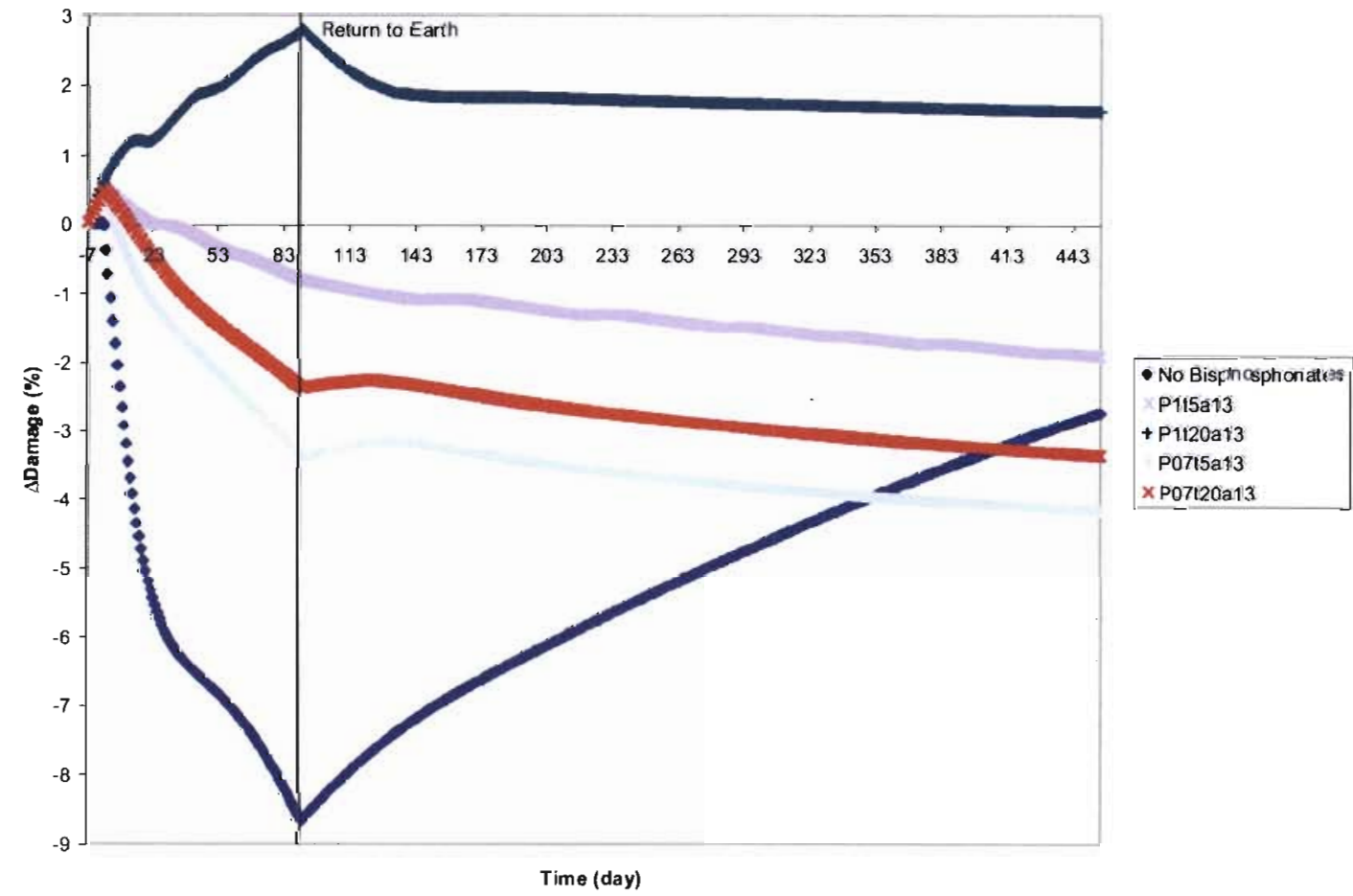

Figure C8. Predicted bisphosphonate effects beginning 7 days preflight on damage accumulation (D) and posttreatment return to Earth from 90-day spaceflight. 


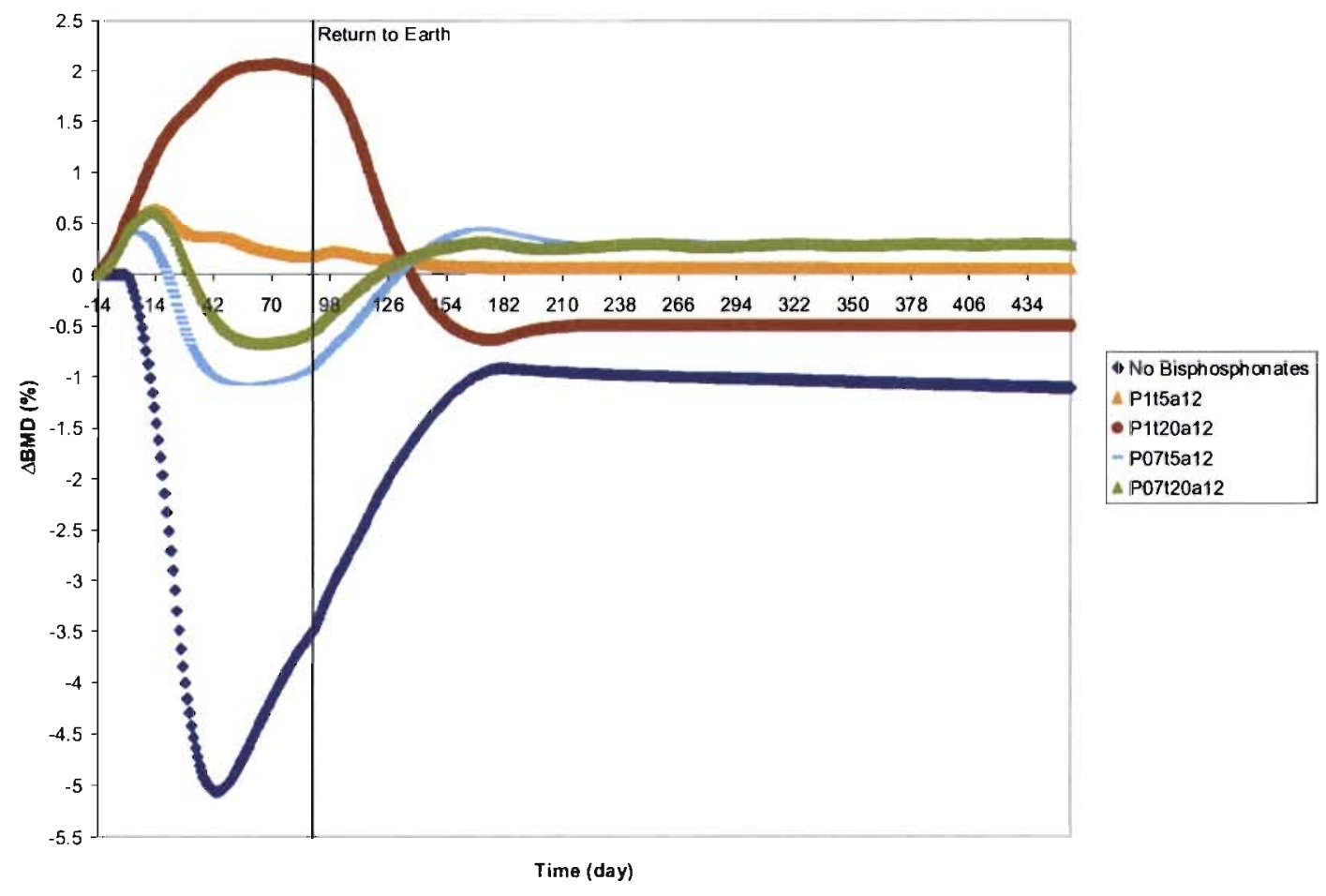

Figure C9. Predicted bisphosphonate effects beginning 14 days preflight on BMD and posttreatment return to Earth from $\mathbf{9 0}$-day spaceflight.

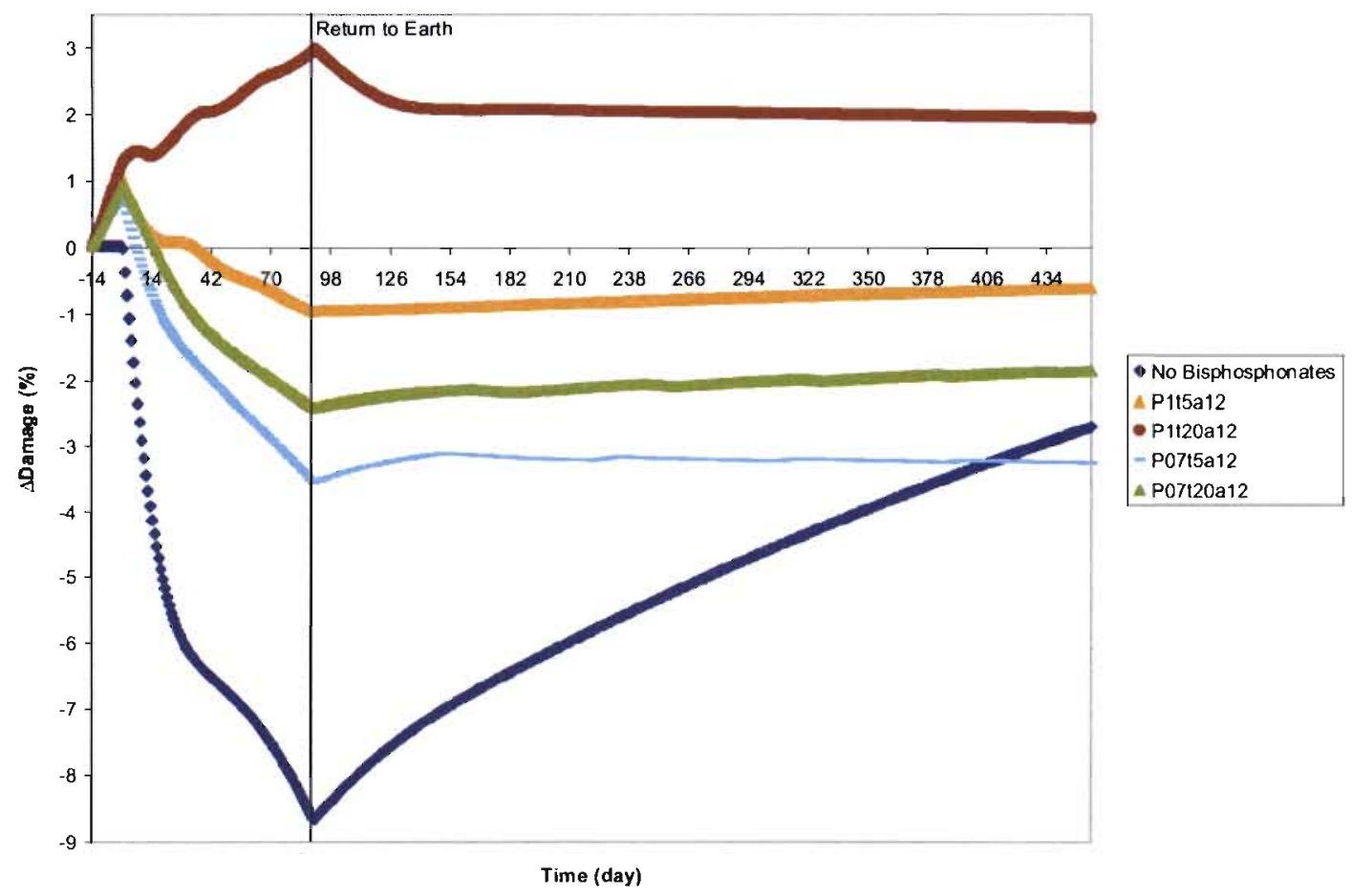

Figure C10. Predicted bisphosphonate effects beginning 14 days preflight on damage accumulation (D) and posttreatment return to Earth from 90-day spaceflight. 


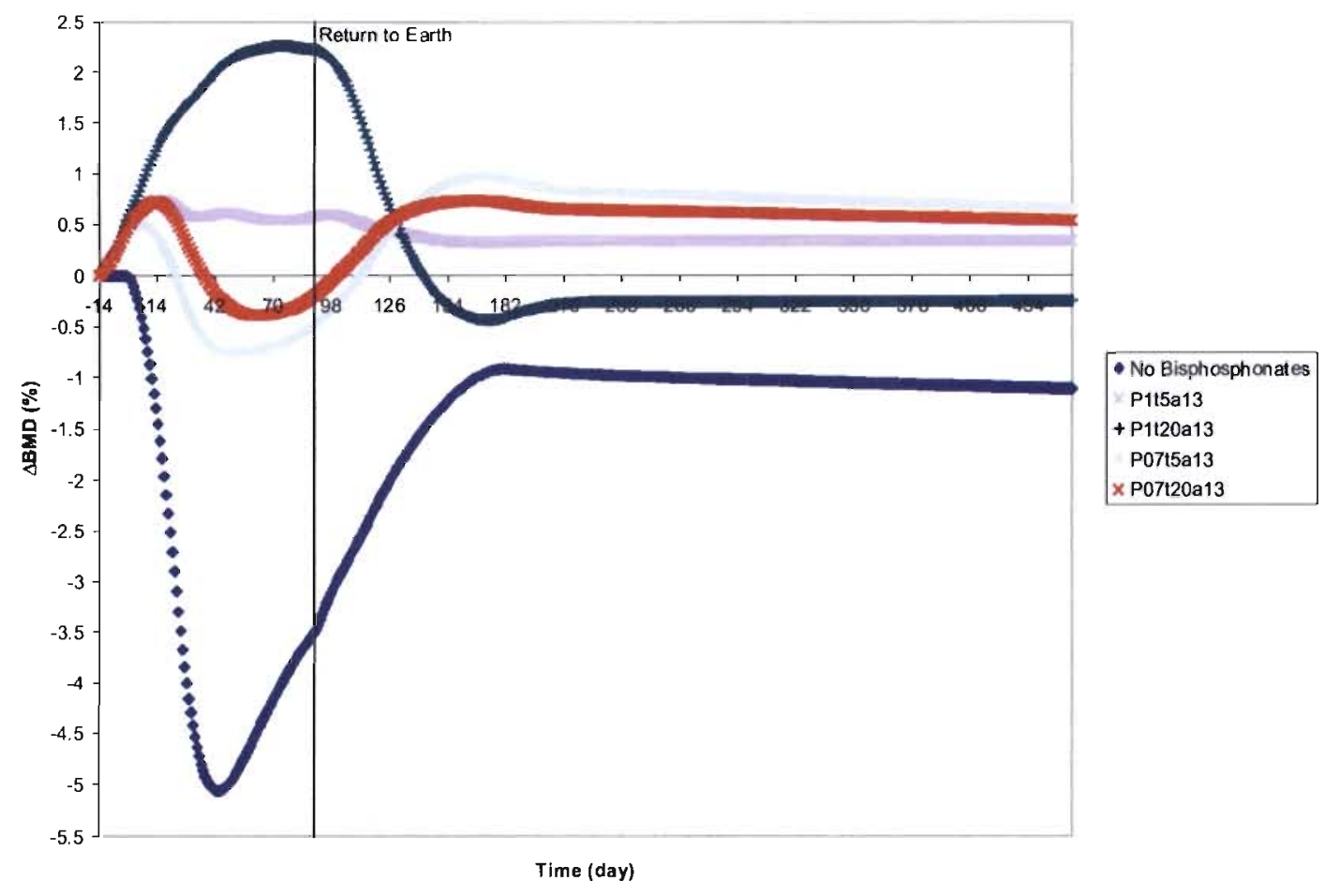

Figure C11. Predicted bisphosphonate effects beginning 14 days preflight on BMD and posttreatment return to Earth from 90-day spaceflight.

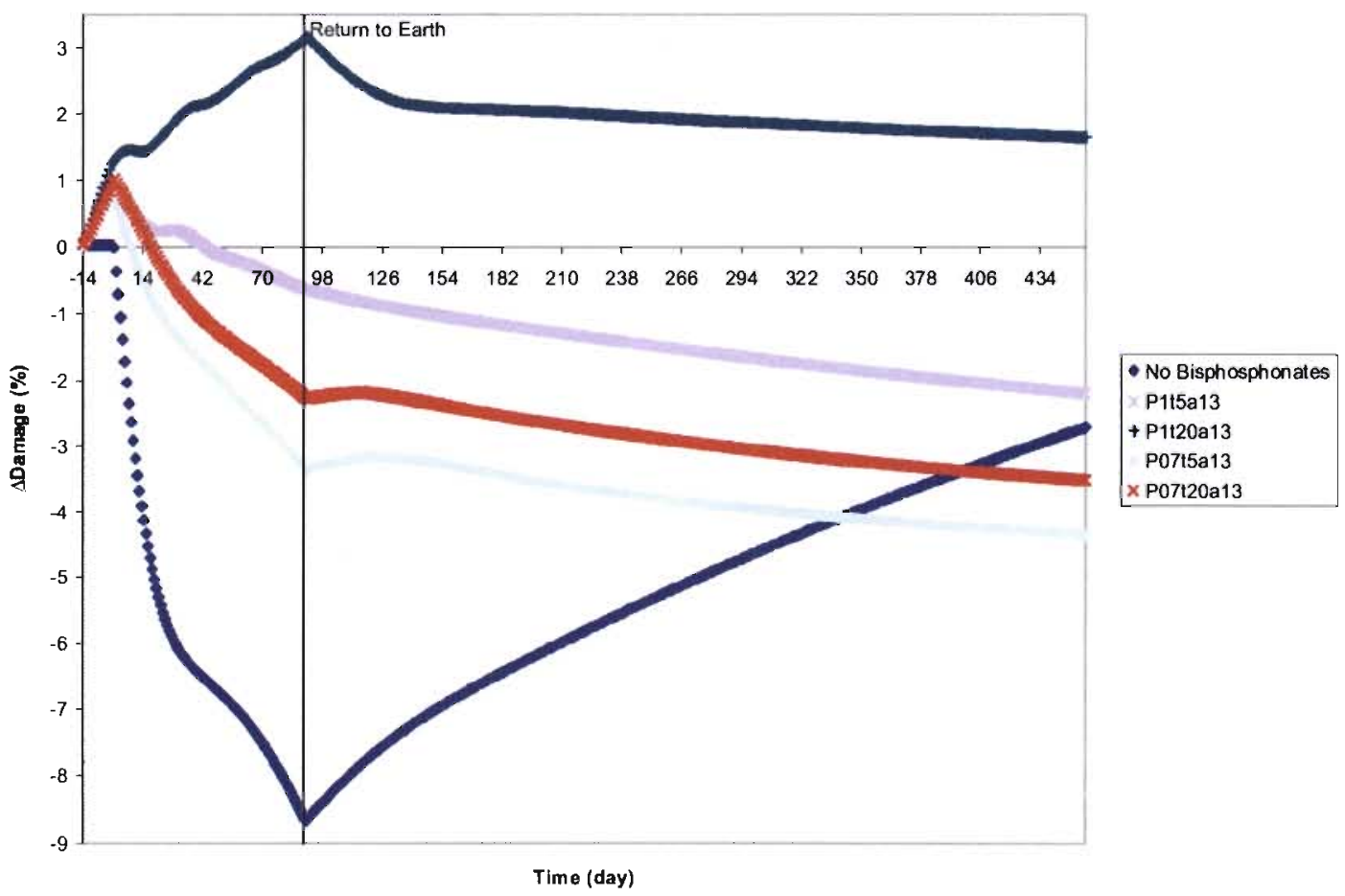

Figure C12. Predicted bisphosphonate effects beginning 14 days preflight on damage accumulation (D) and posttreatment return to Earth from 90-day spaceflight. 


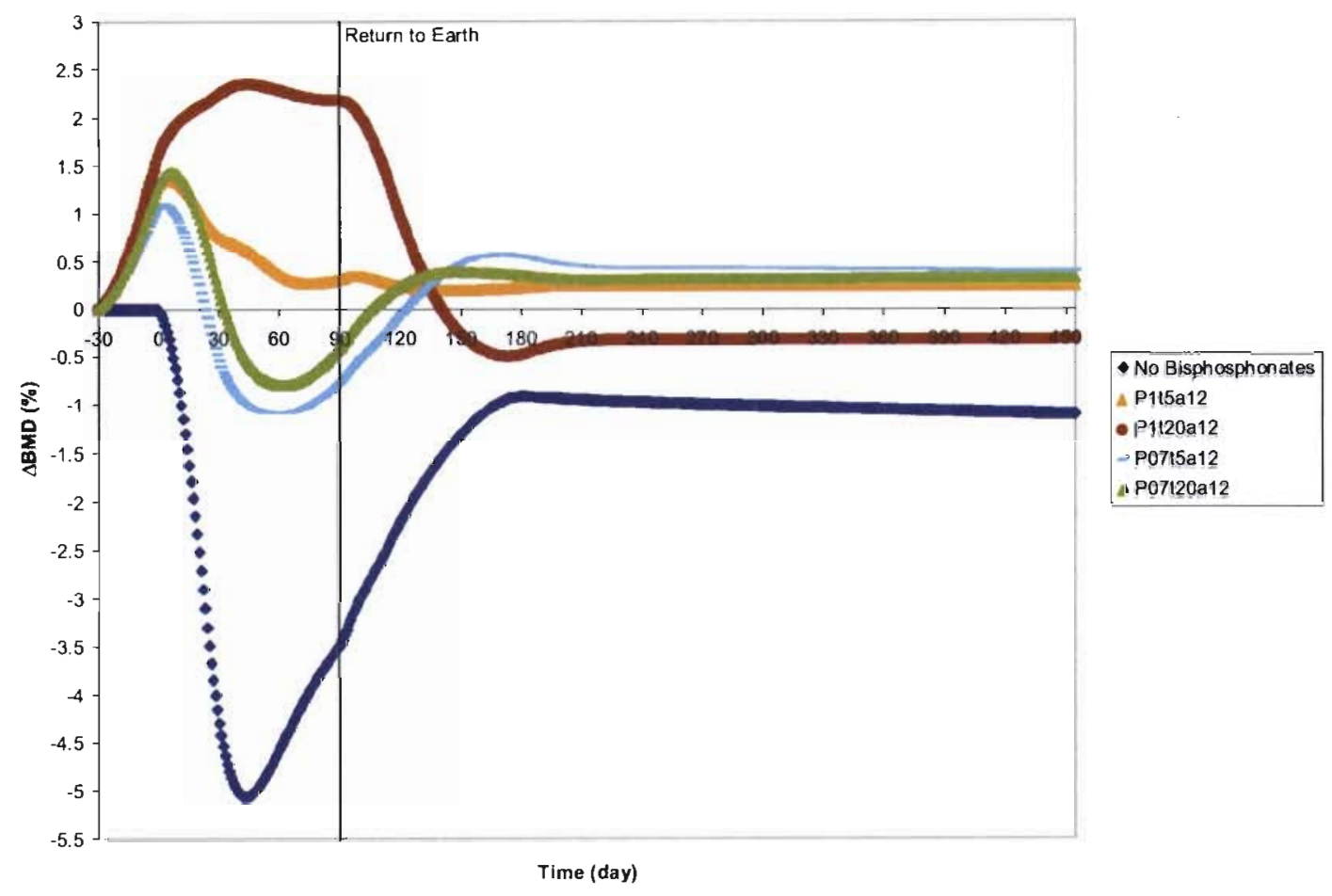

Figure C13. Predicted bisphosphonate effects beginning 30 days preflight on BMD and posttreatment return to Earth from 90-day spaceflight.

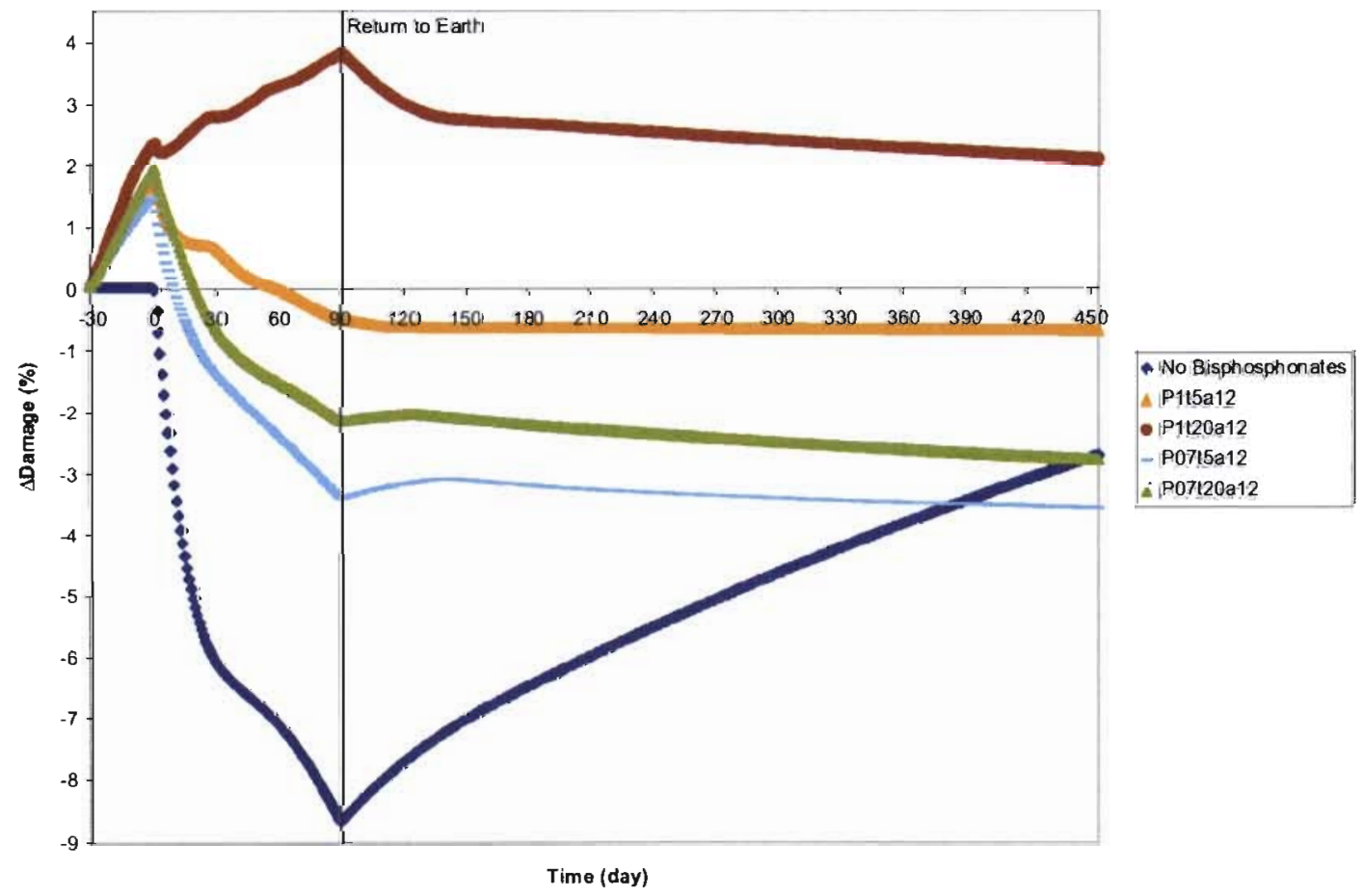

Figure C14. Predicted bisphosphonate effects beginning 30 days preflight on damage accumulation (D) and posttreatment return to Earth from 90-day spaceflight. 


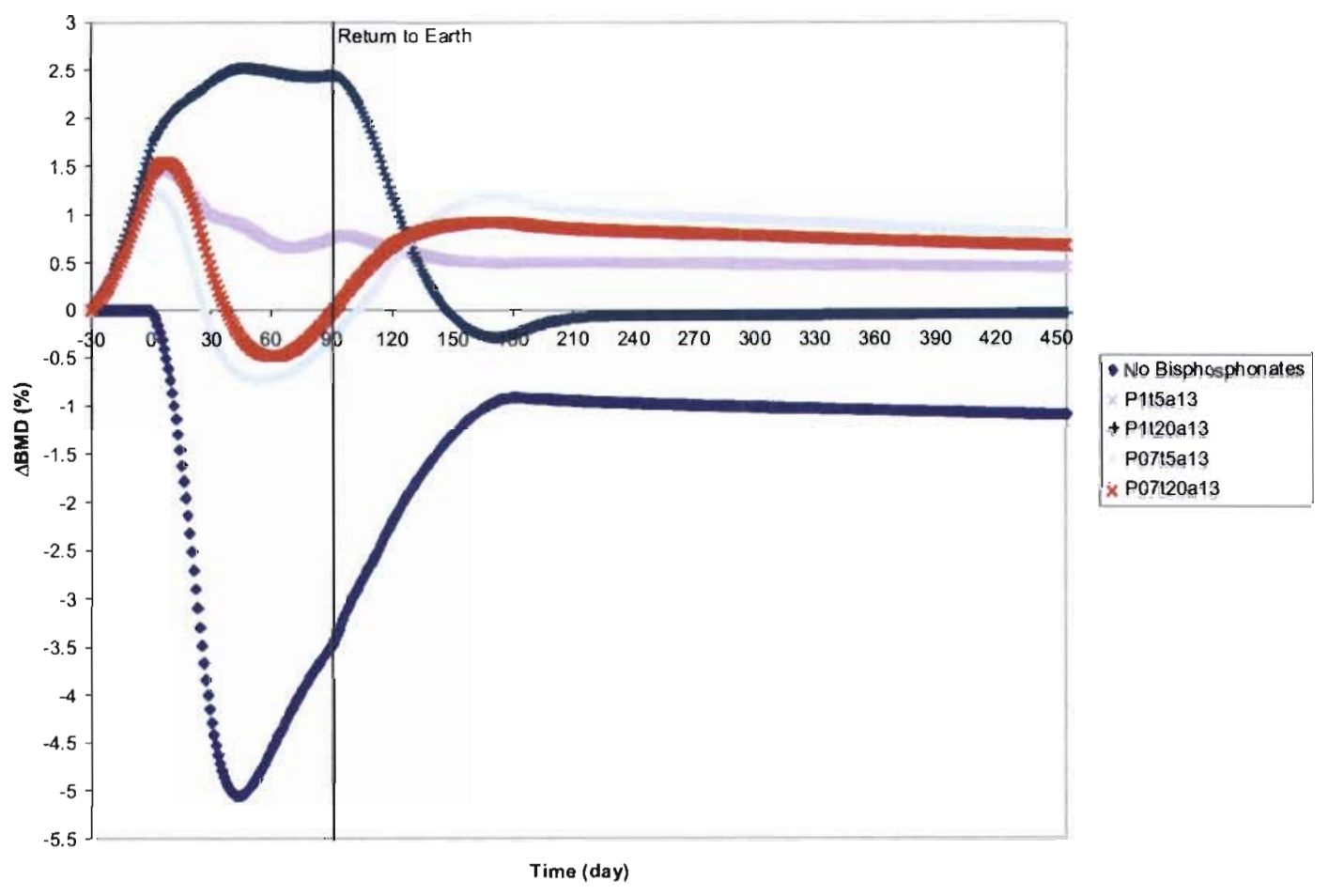

Figure C15. Predicted bisphosphonate effects beginning 30 days preflight on BMD and posttreatment return to Earth from 90-day spaceflight.

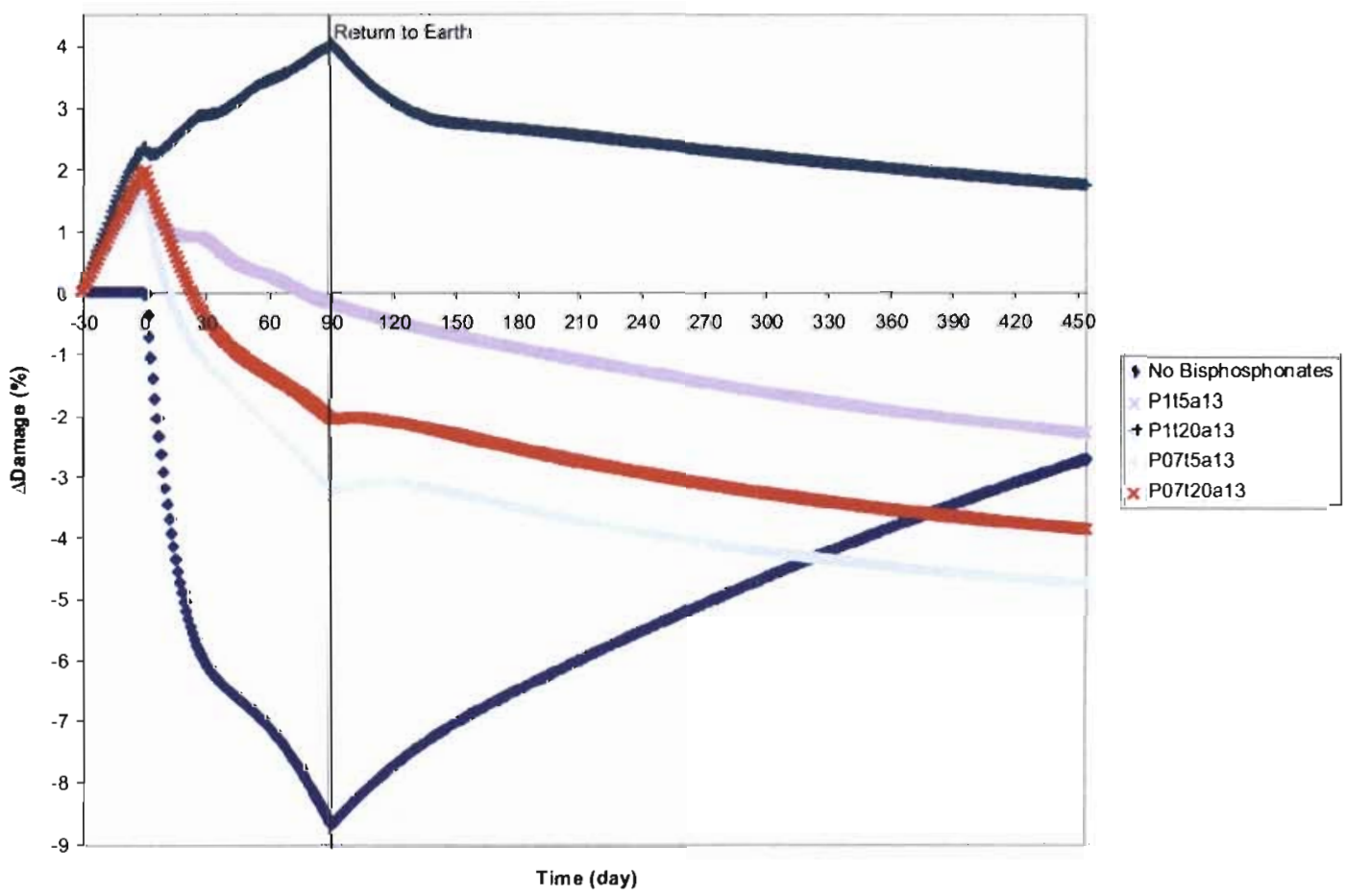

Figure C16. Predicted bisphosphonate effects beginning 30 days preflight on damage accumulation (D) and posttreatment return to Earth from 90-day spaceflight. 


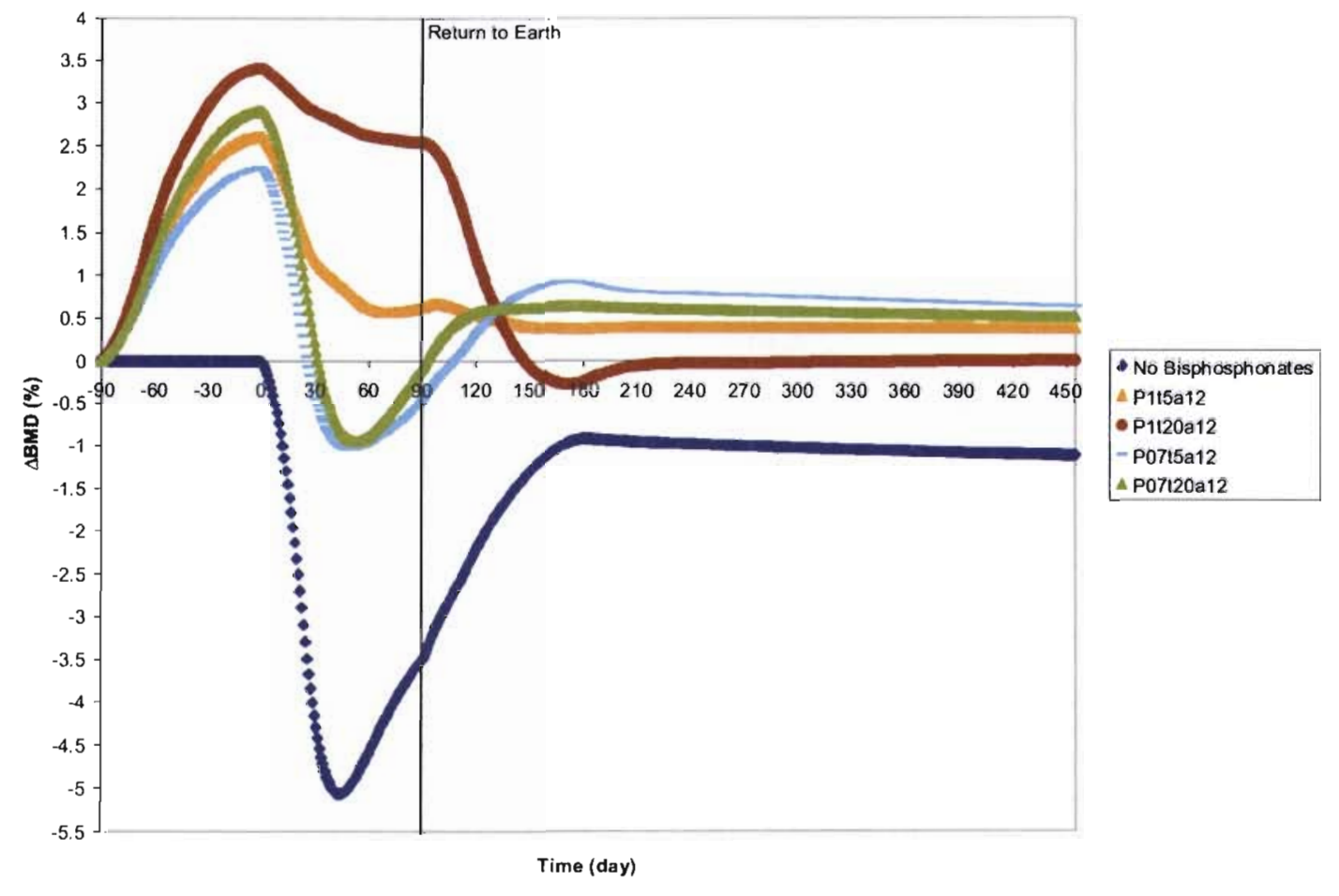

Figure C17. Predicted bisphosphonate effects beginning 90 days preflight on BMD and posttreatment return to Earth from 90-day spaceflight.

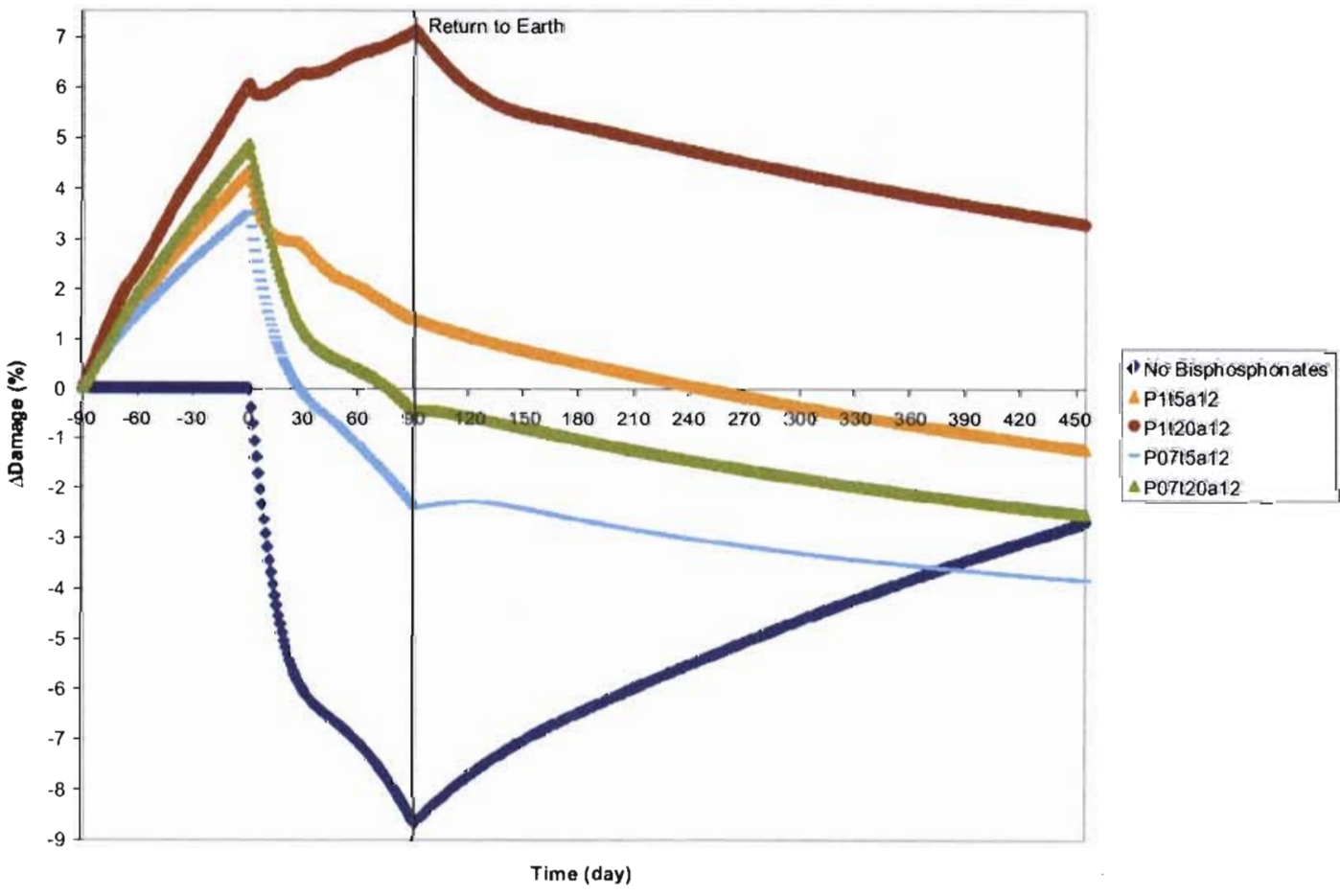

Figure C18. Predicted bisphosphonate effects beginning 90 days preflight on damage accumulation (D) and posttreatment return to Earth from 90-day spaceflight. 


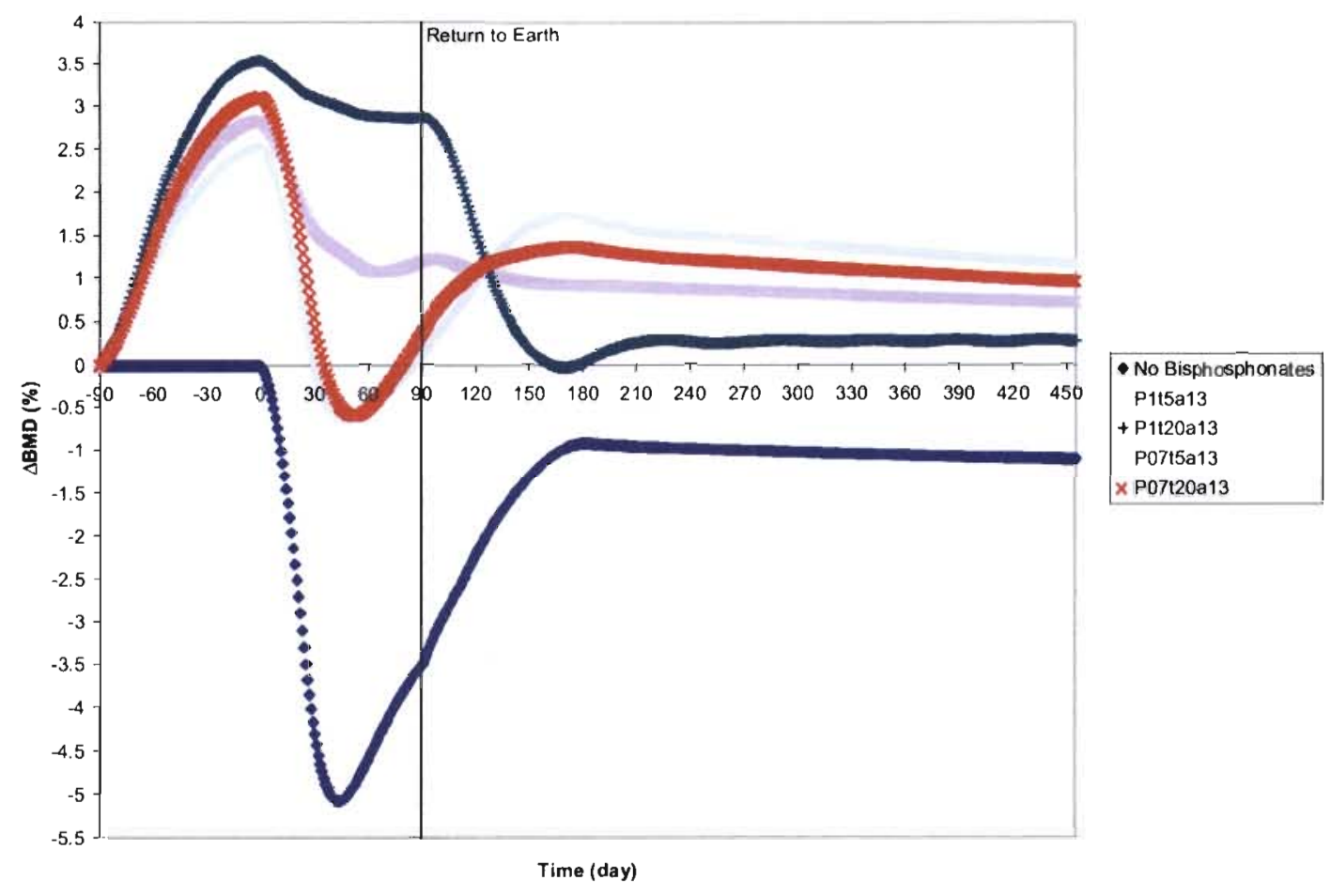

Figure C19. Predicted bisphosphonate effects beginning 90 days preflight on BMD and posttreatment return to Earth from $\mathbf{9 0 - d a y ~ s p a c e f l i g h t . ~}$

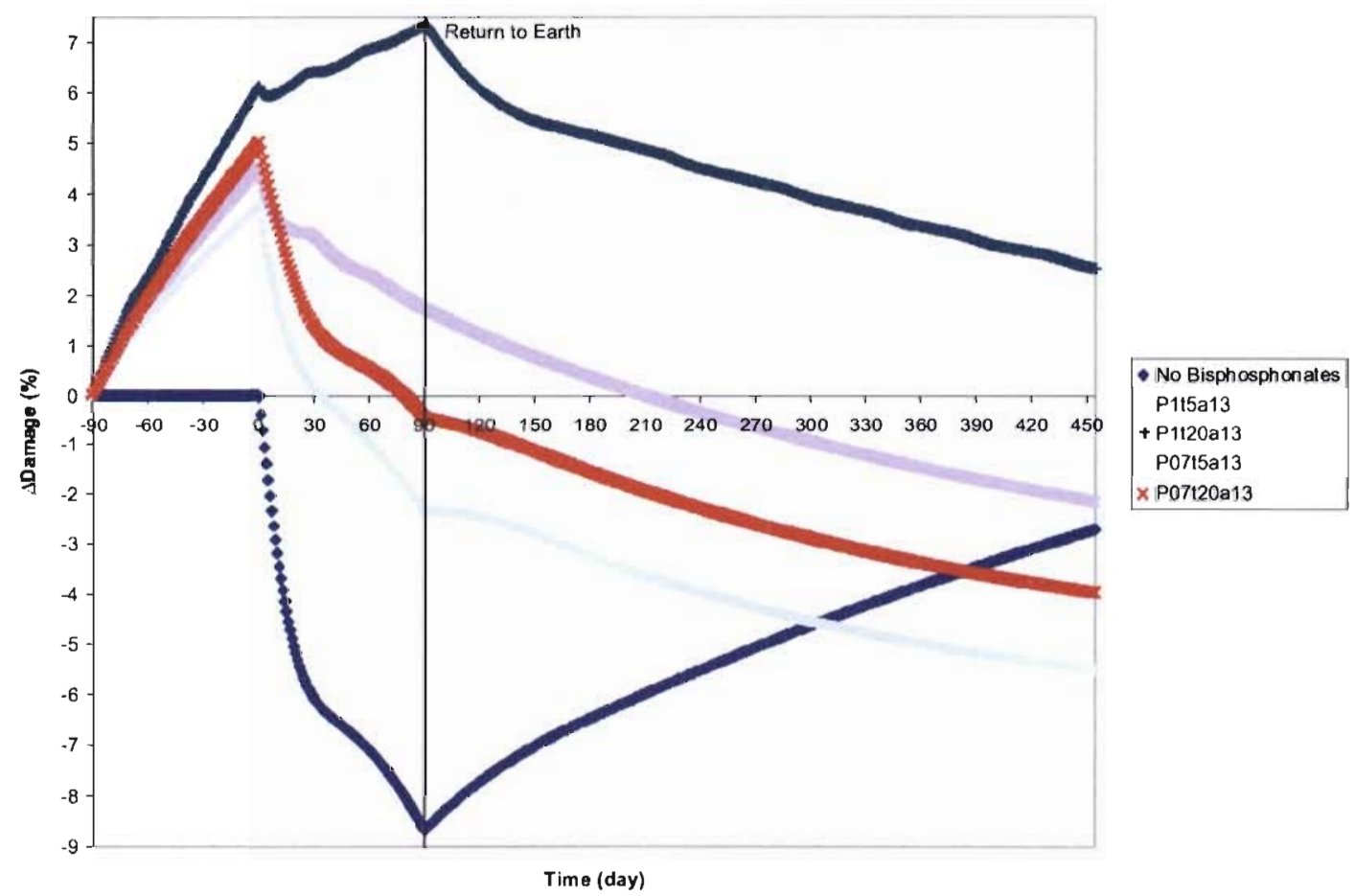

Figure C20. Predicted bisphosphonate effects beginning 90 days preflight on damage accumulation (D) and posttreatment return to Earth from 90-day spaceflight. 


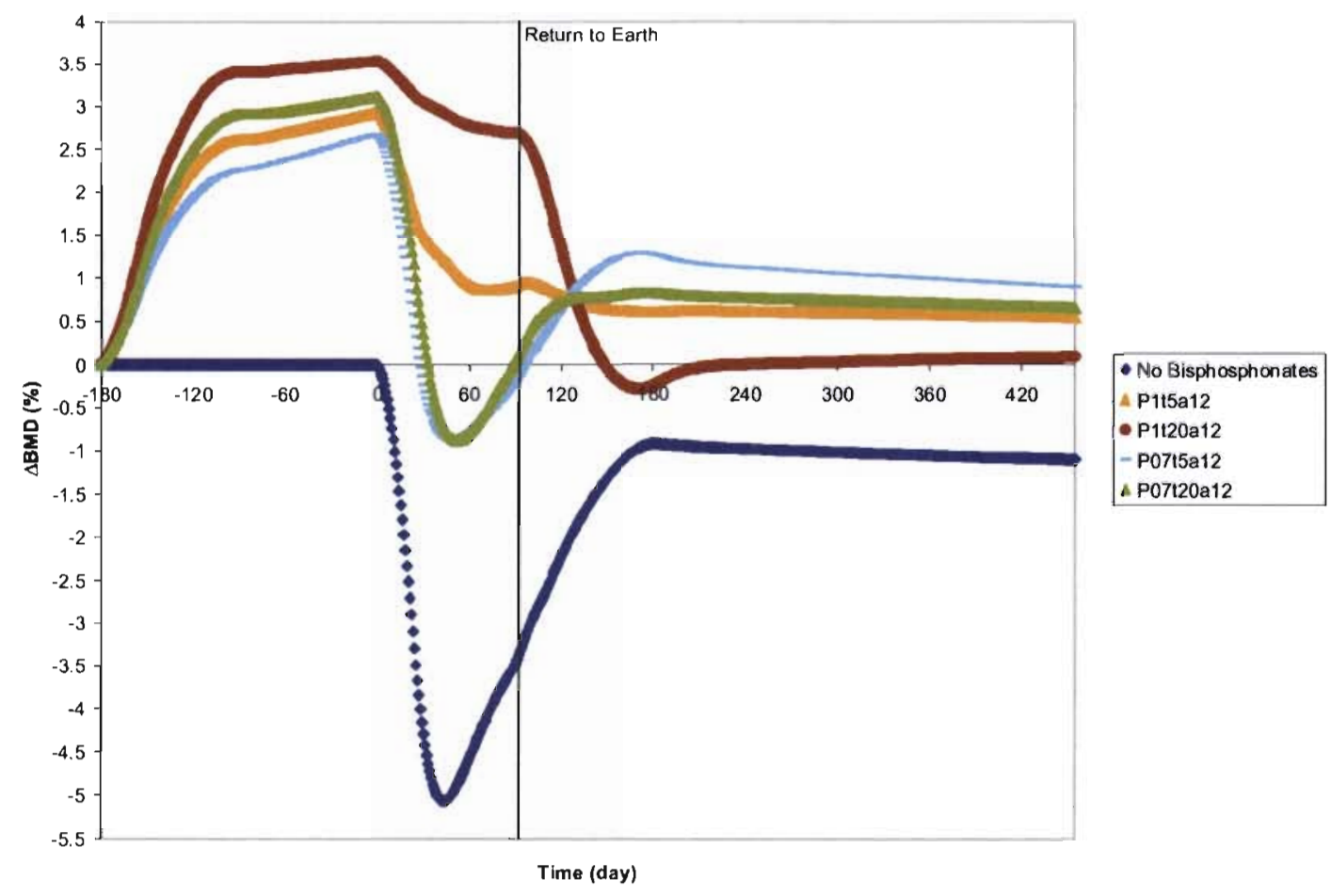

Figure C21. Predicted bisphosphonate effects beginning 180 days preflight on BMD and posttreatment return to Earth from $\mathbf{9 0 - d a y ~ s p a c e f l i g h t . ~}$

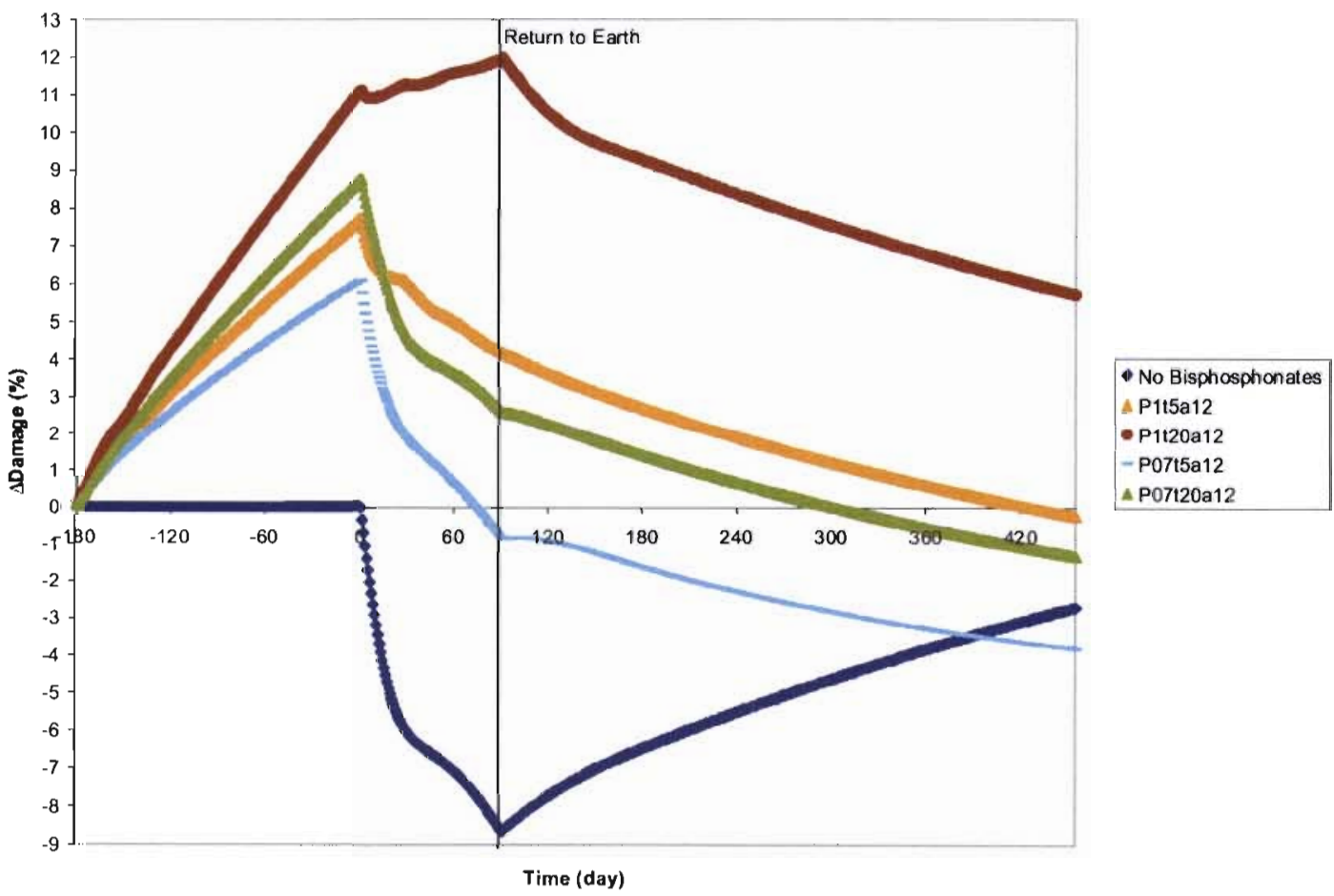

Figure C22. Predicted bisphosphonate effects beginning 180 days preflight on damage accumulation (D) and posttreatment return to Earth from 90-day spaceflight. 


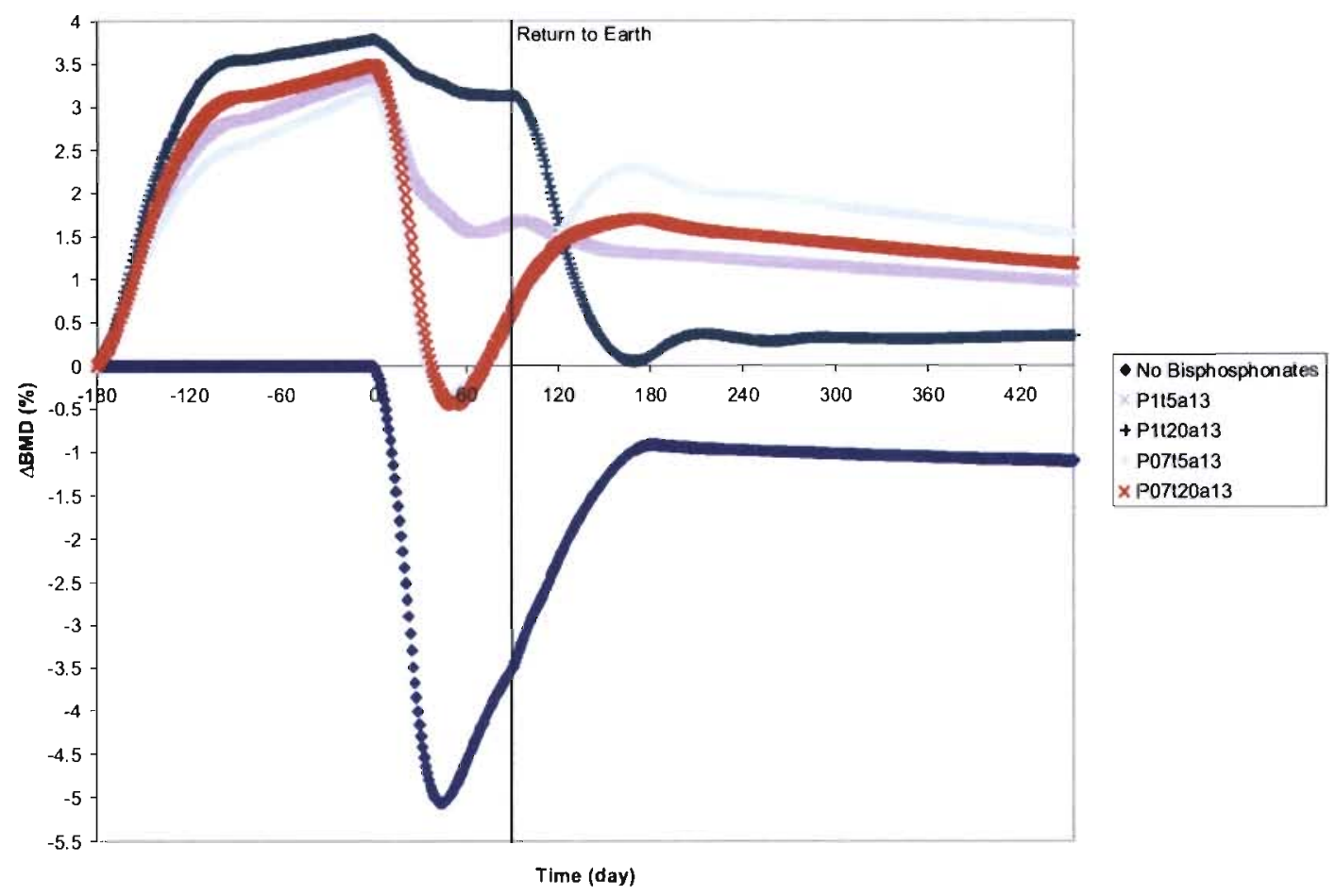

Figure C23. Predicted bisphosphonate effects beginning 180 days preflight on BMD and posttreatment return to Earth from 90-day spaceflight.

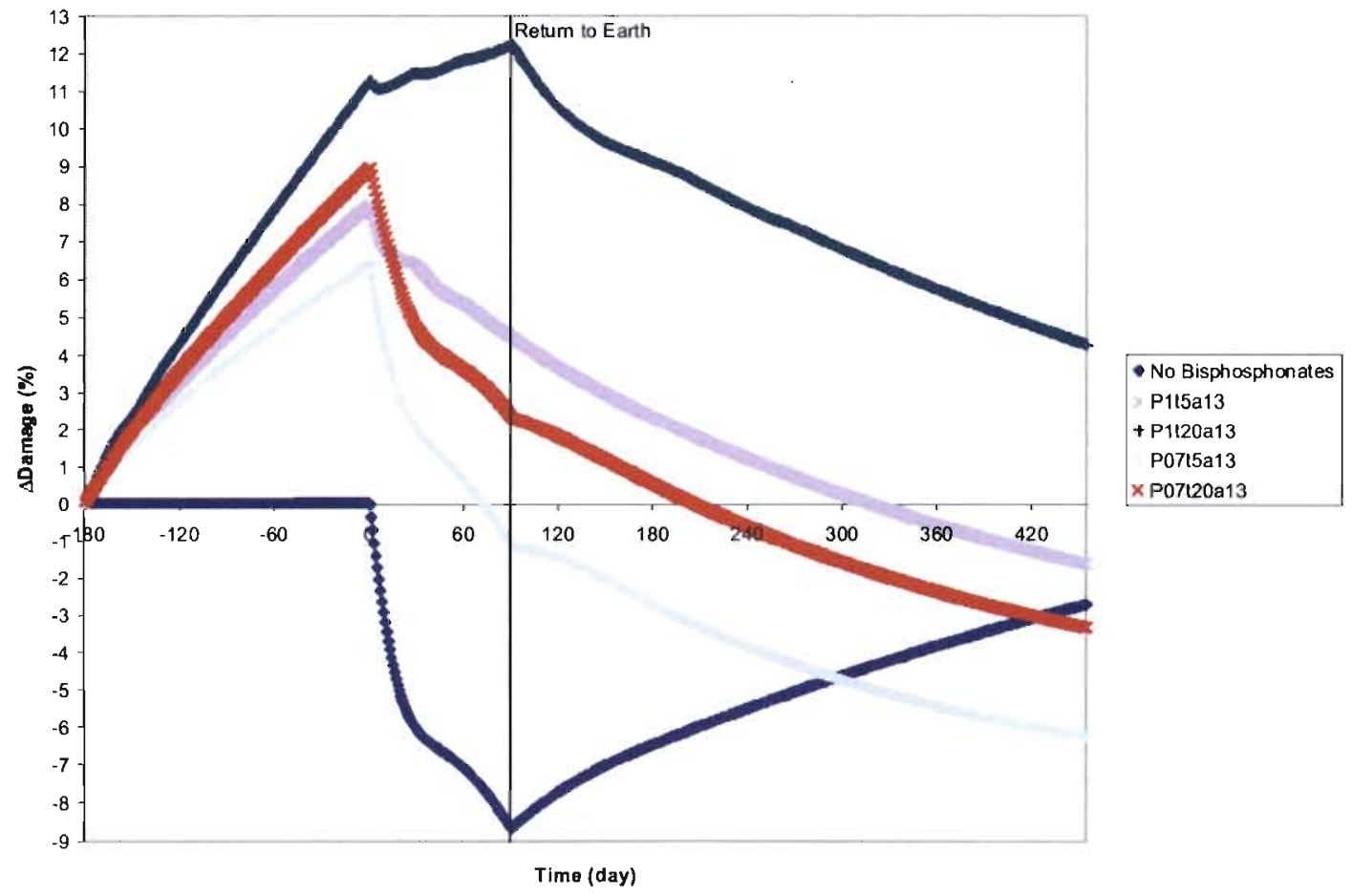

Figure C24. Predicted bisphosphonate effects beginning 180 days preflight on damage accumulation (D) and posttreatment return to Earth from 90-day spaceflight. 


\section{APPENDIX D: FIGURES (180-DAY SPACEFLIGHT)}

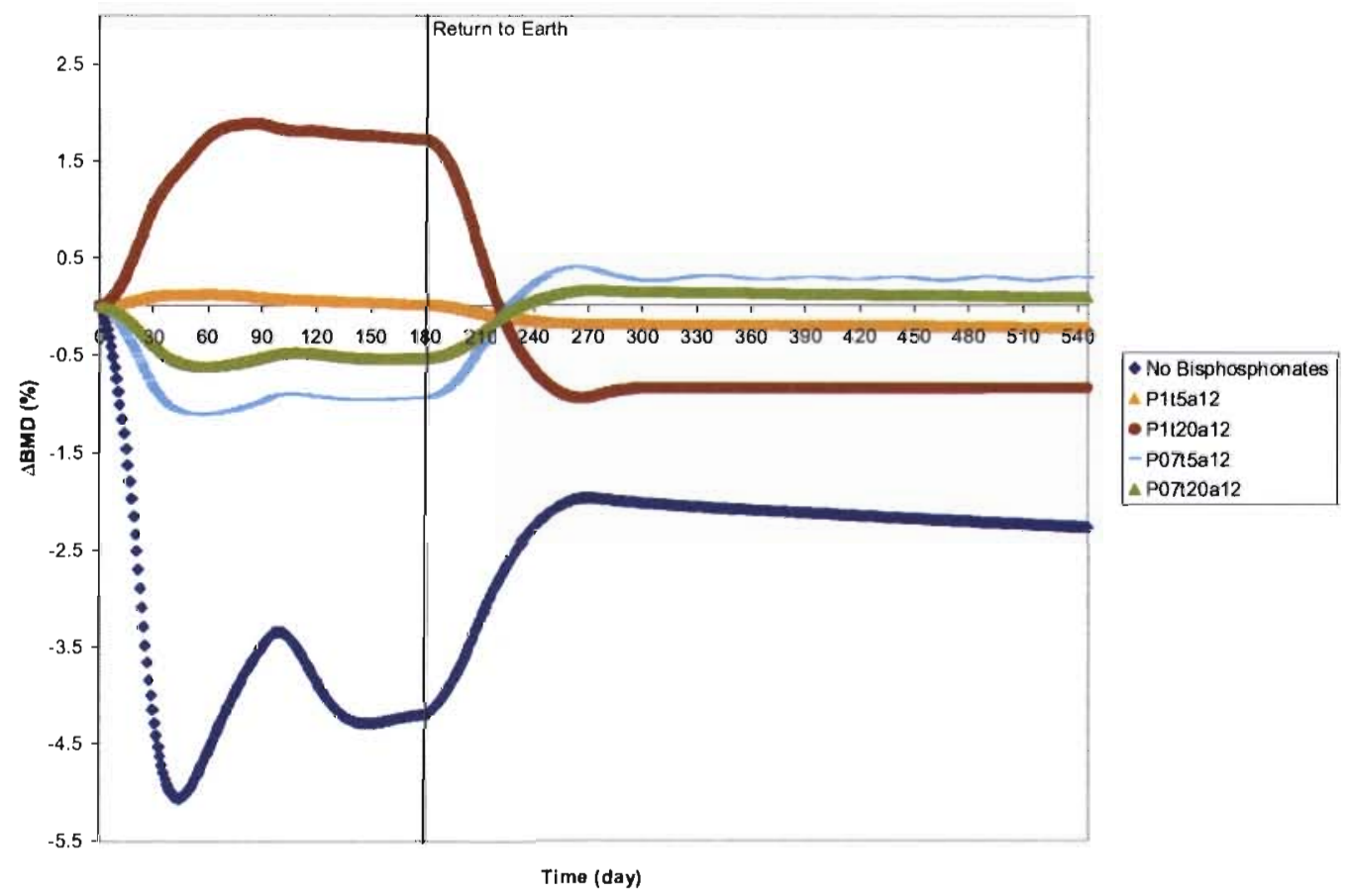

Figure D1. Predicted bisphosphonate effects on BMD and posttreatment return to Earth from 180day spaceflight.

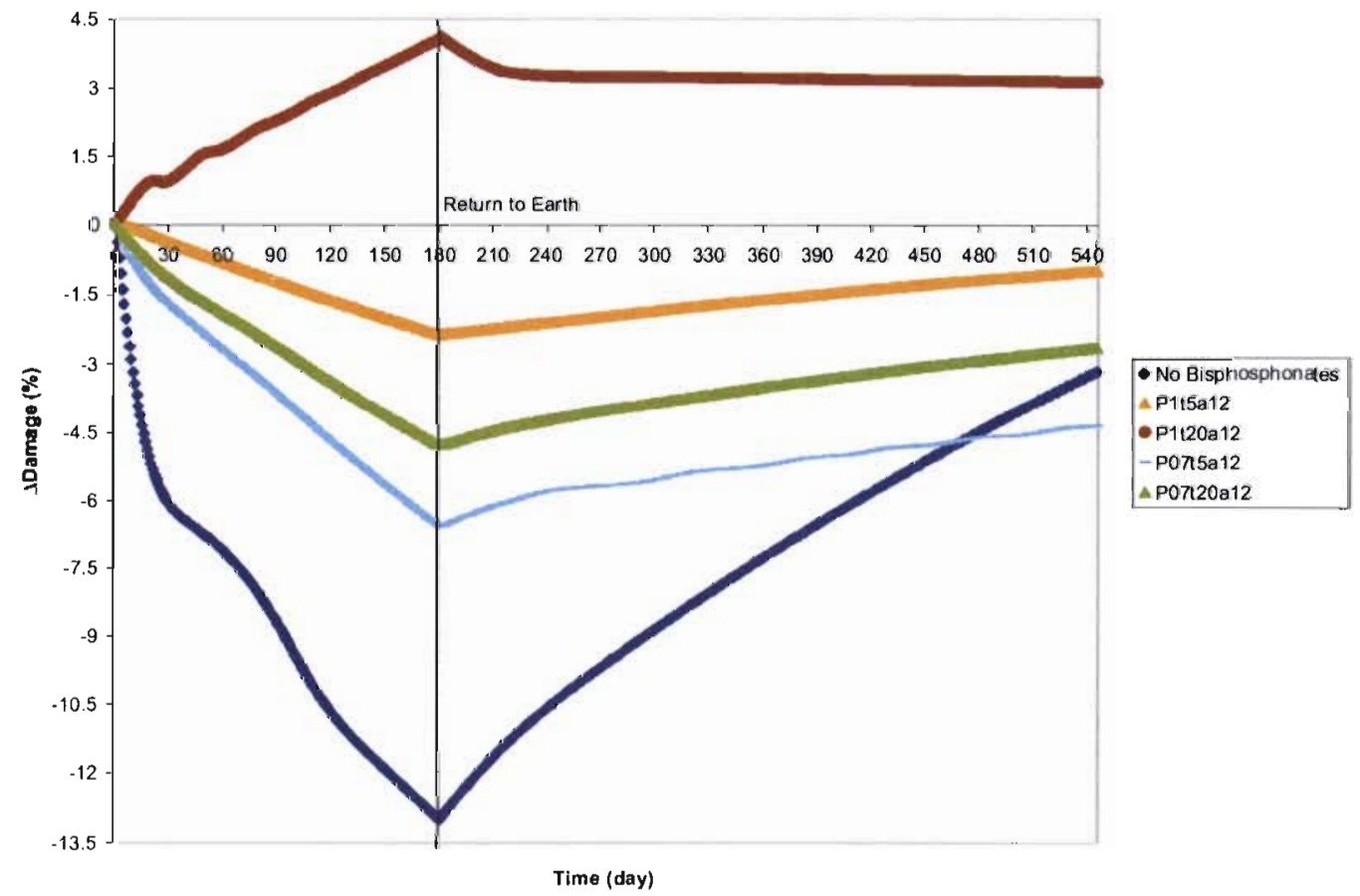

Figure D2. Predicted bisphosphonate effects on damage accumulation (D) and posttreatment return to Earth from 180-day spaceflight. 


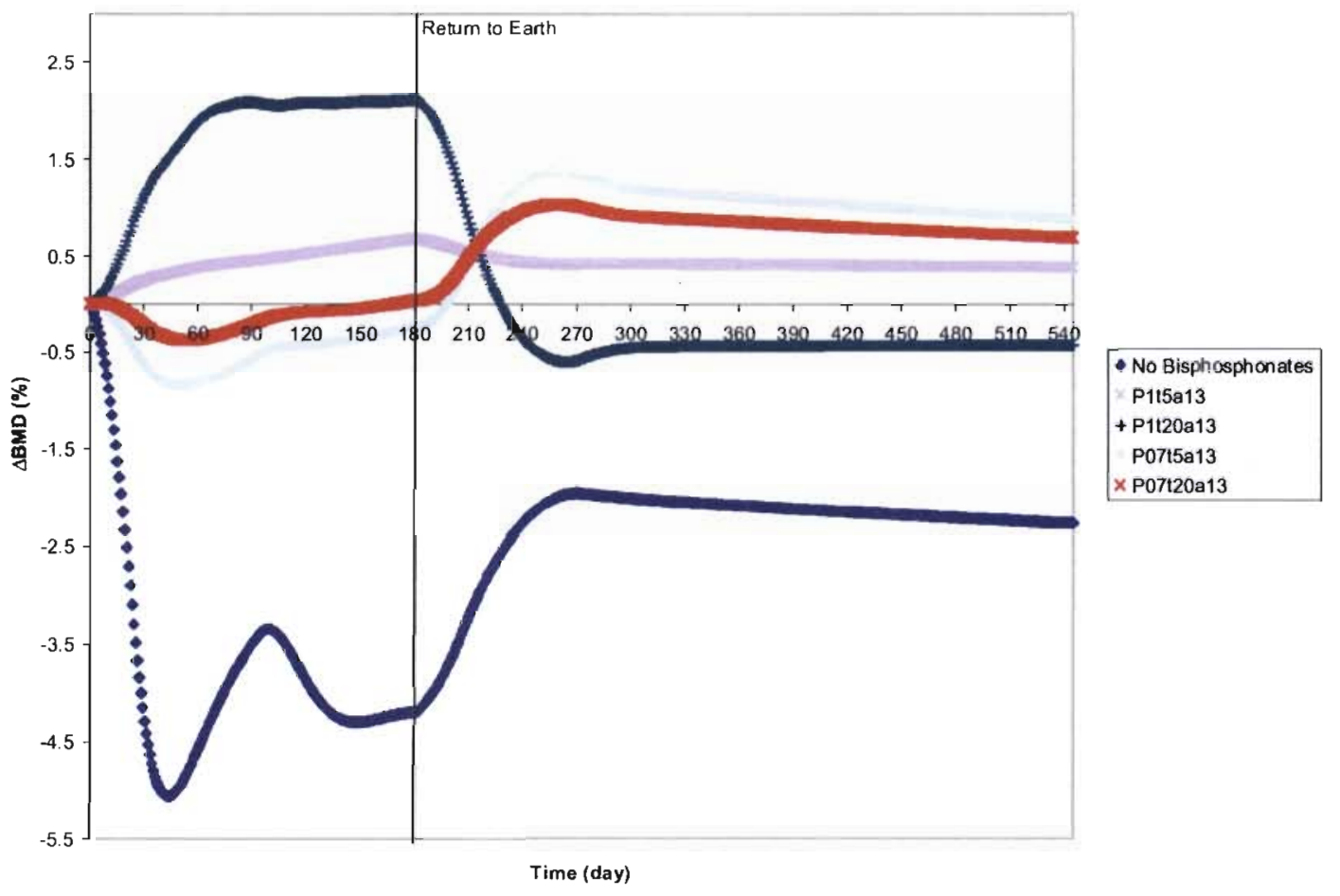

Figure D3. Predicted bisphosphonate effects on BMD and posttreatment return to Earth from 180day spaceflight.

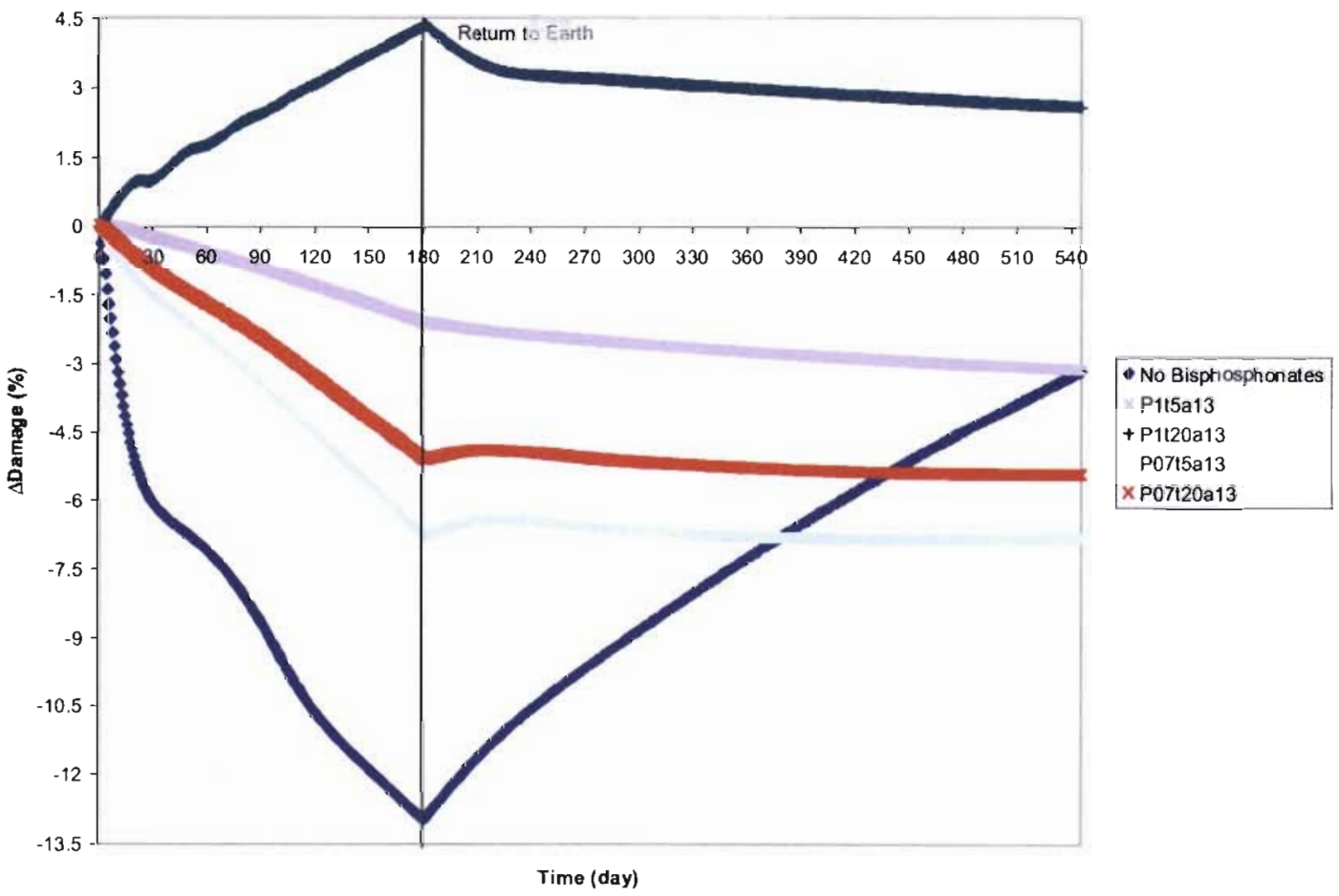

Figure D4. Predicted bisphosphonate effects on damage accumulation (D) and posttreatment return to Earth from 180-day spaceflight. 


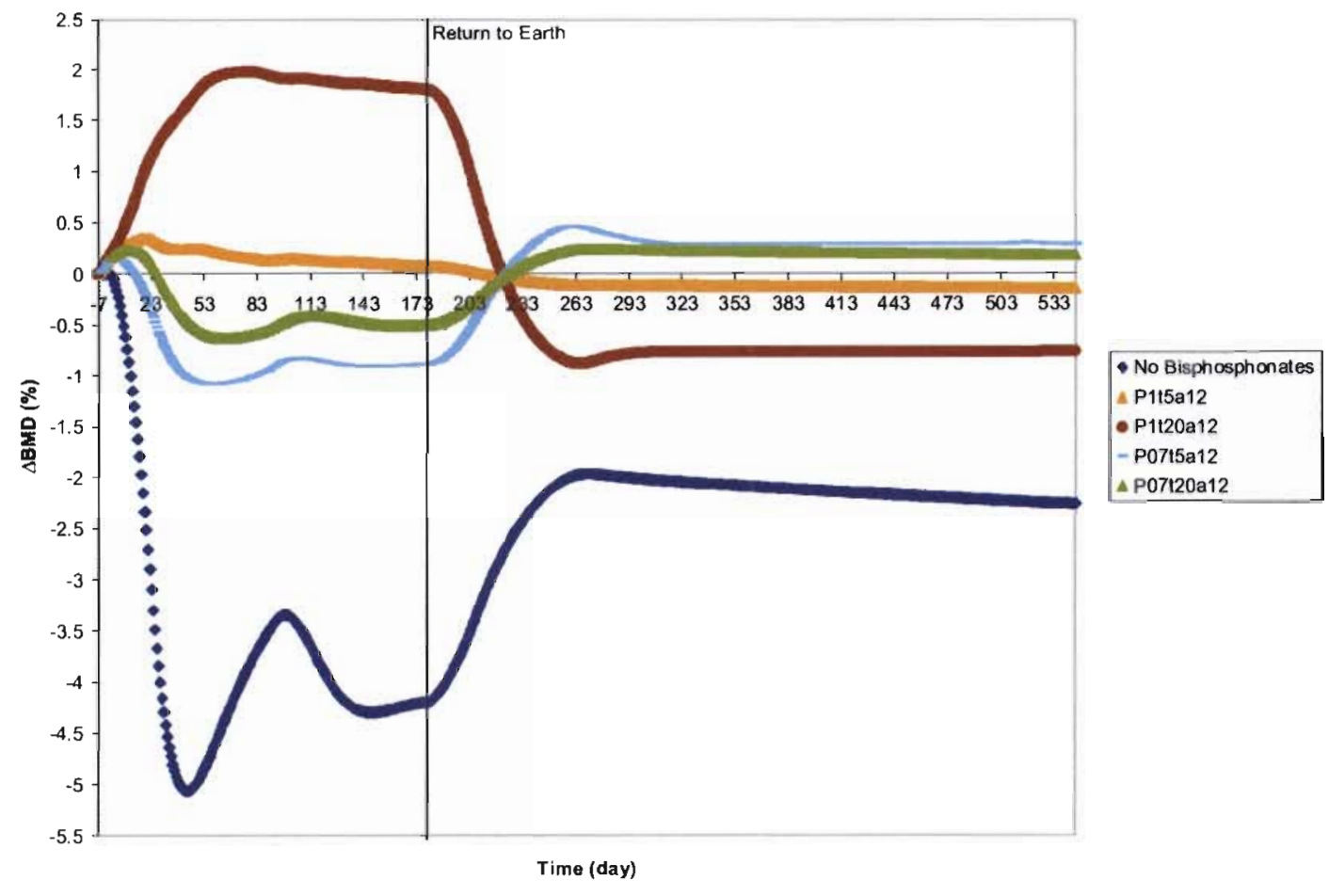

Figure D5. Predicted bisphosphonate effects beginning 7 days preflight on BMD and posttreatment return to Earth from 180-day spaceflight.

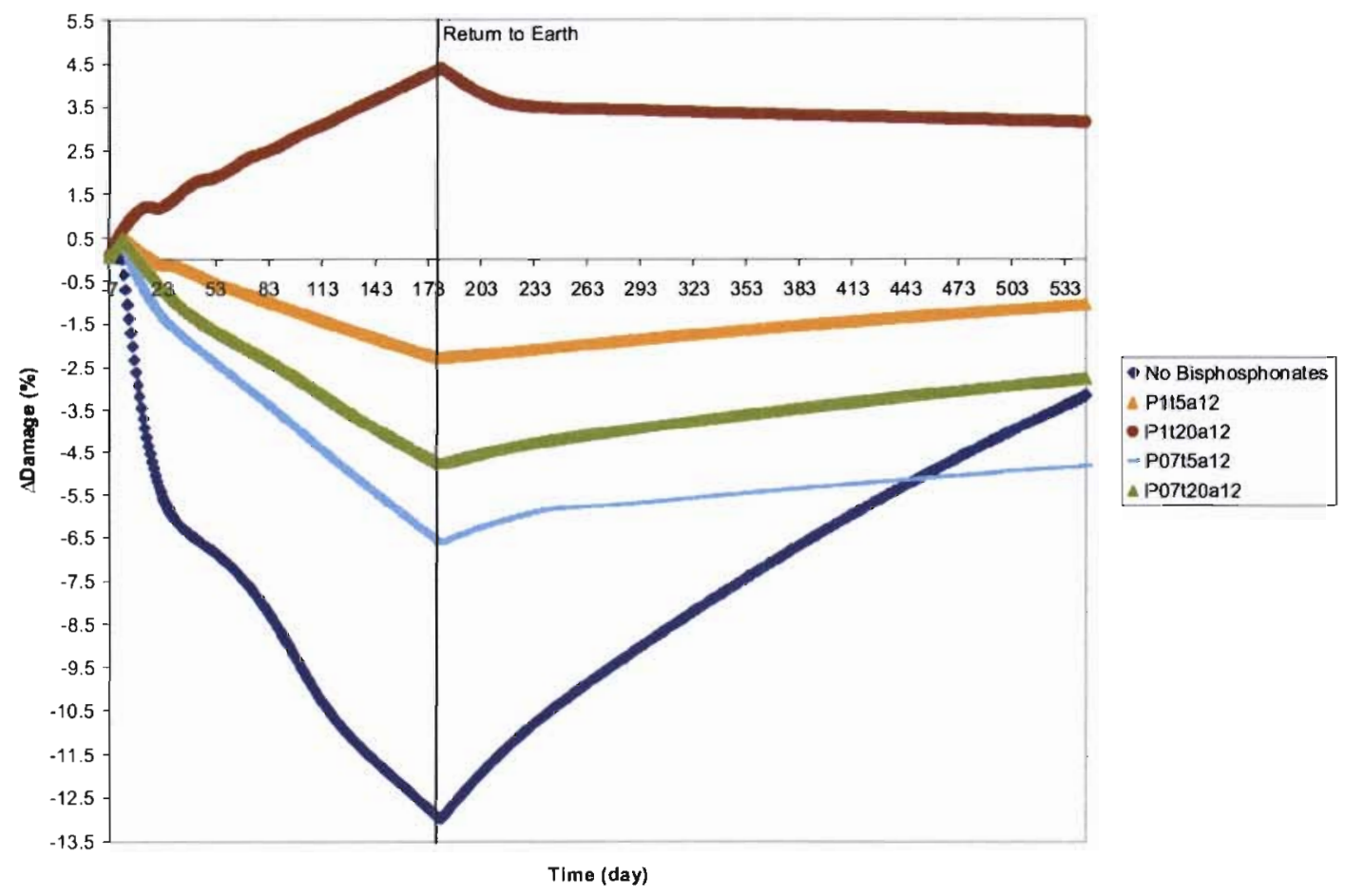

Figure D6. Predicted bisphosphonate effects beginning 7 days preflight on damage accumulation (D) and posttreatment return to Earth from 180-day spaceflight. 


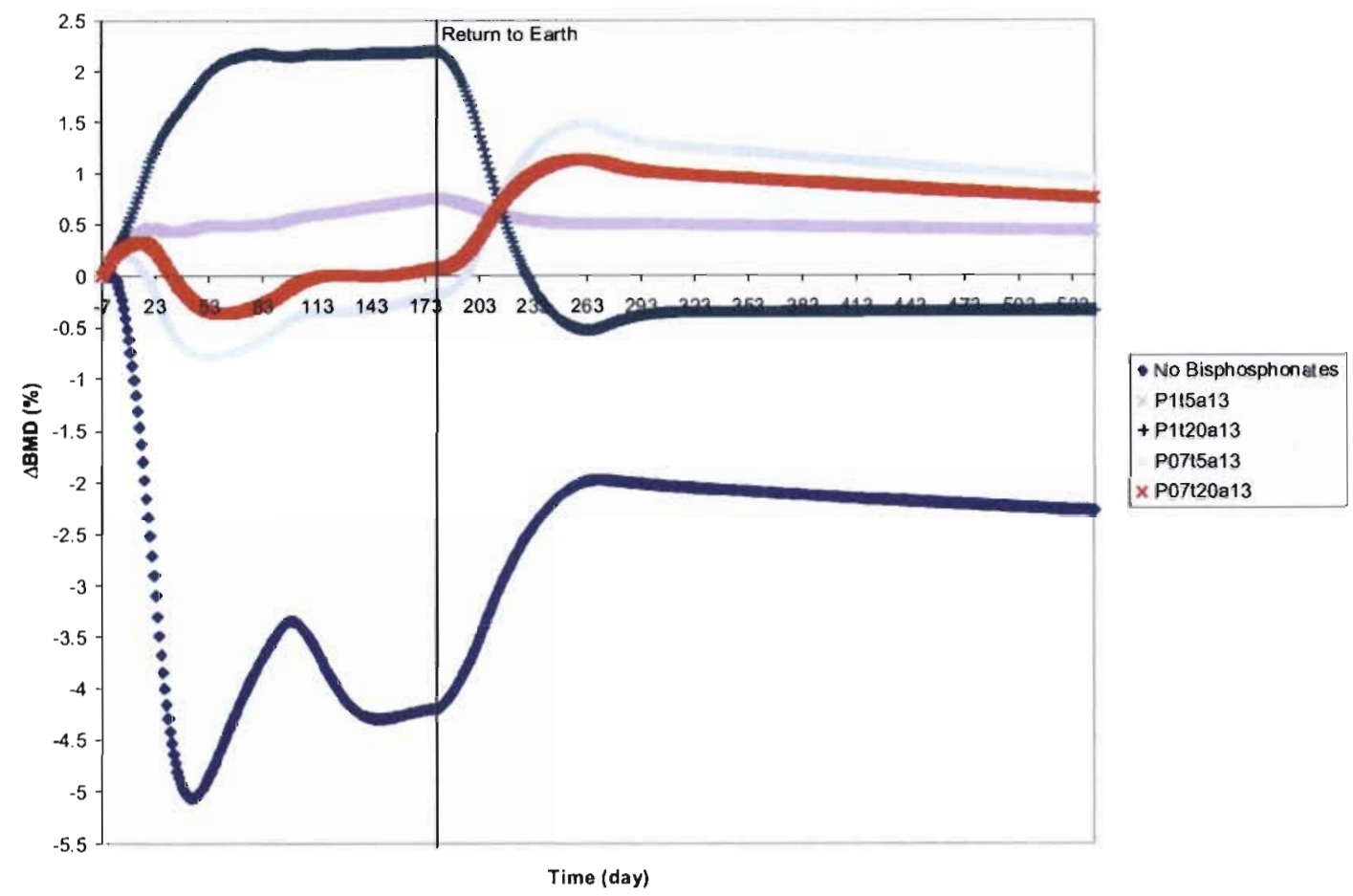

Figure D7. Predicted bisphosphonate effects beginning 7 days preflight on BMD and posttreatment return to Earth from 180-day spaceflight.

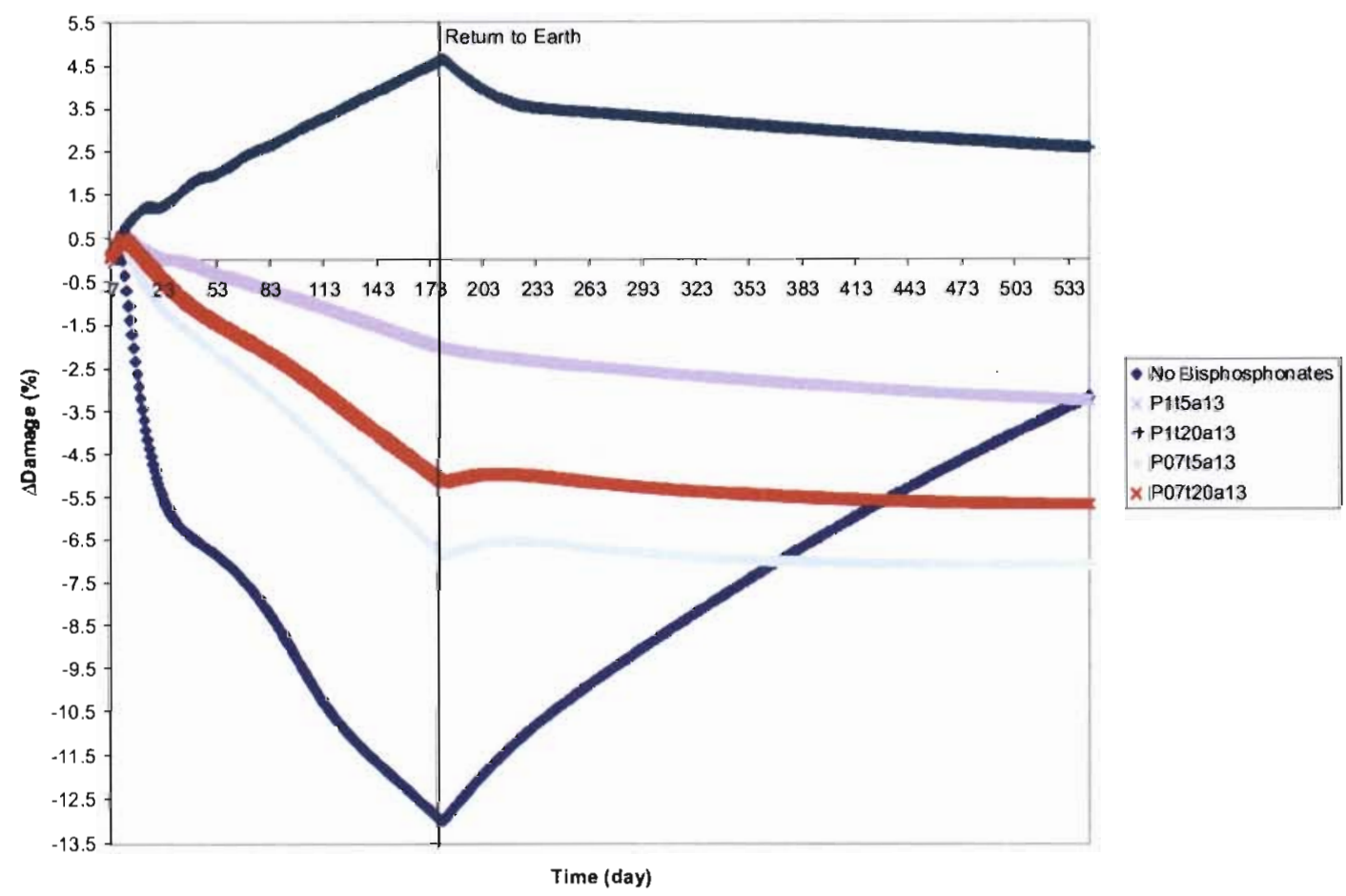

Figure D8. Predicted bisphosphonate effects beginning 7 days preflight on damage accumulation (D) and posttreatment return to Earth from 180-day spaceflight. 


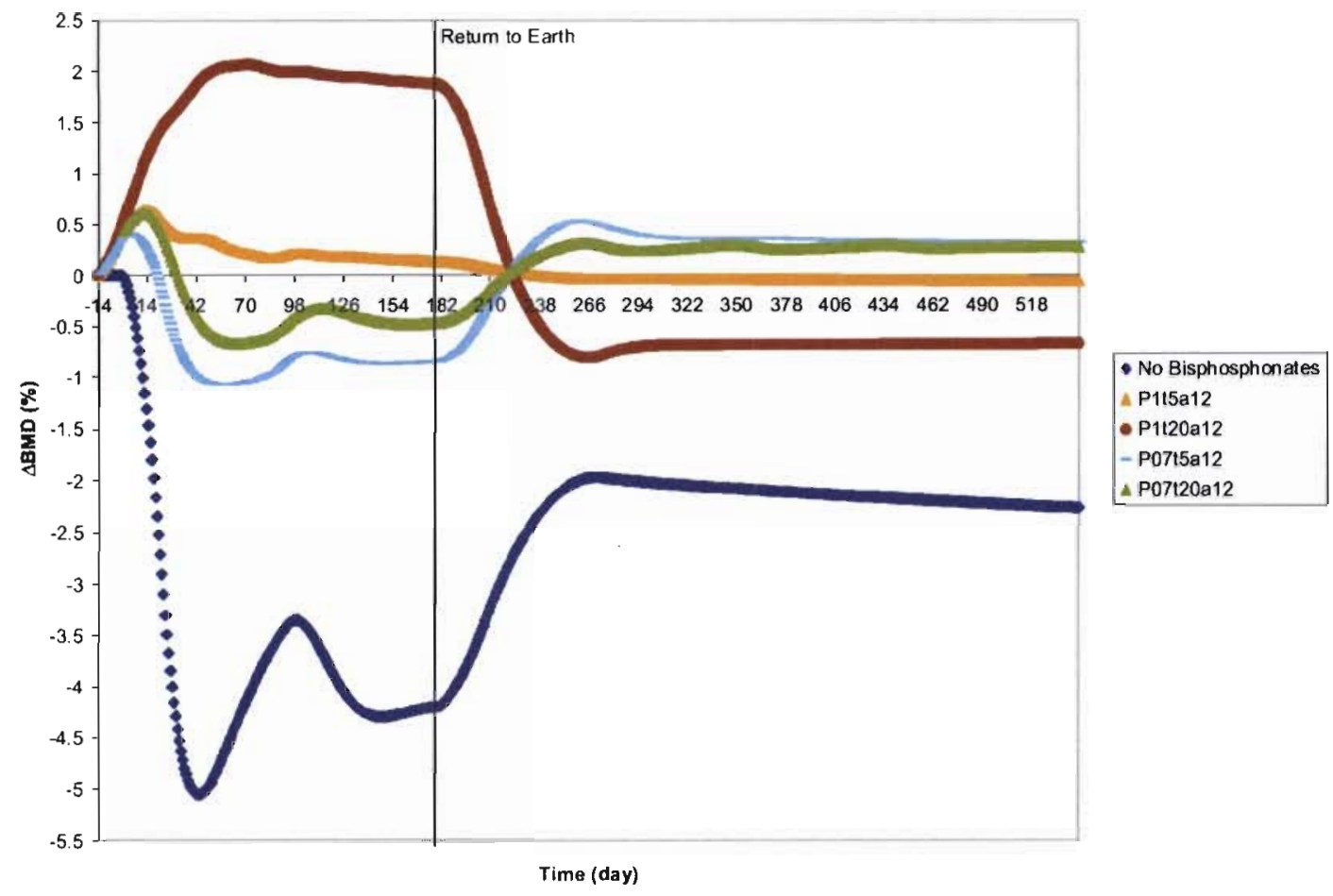

Figure D9. Predicted bisphosphonate effects beginning 14 days preflight on BMD and posttreatment return to Earth from 180-day spaceflight.

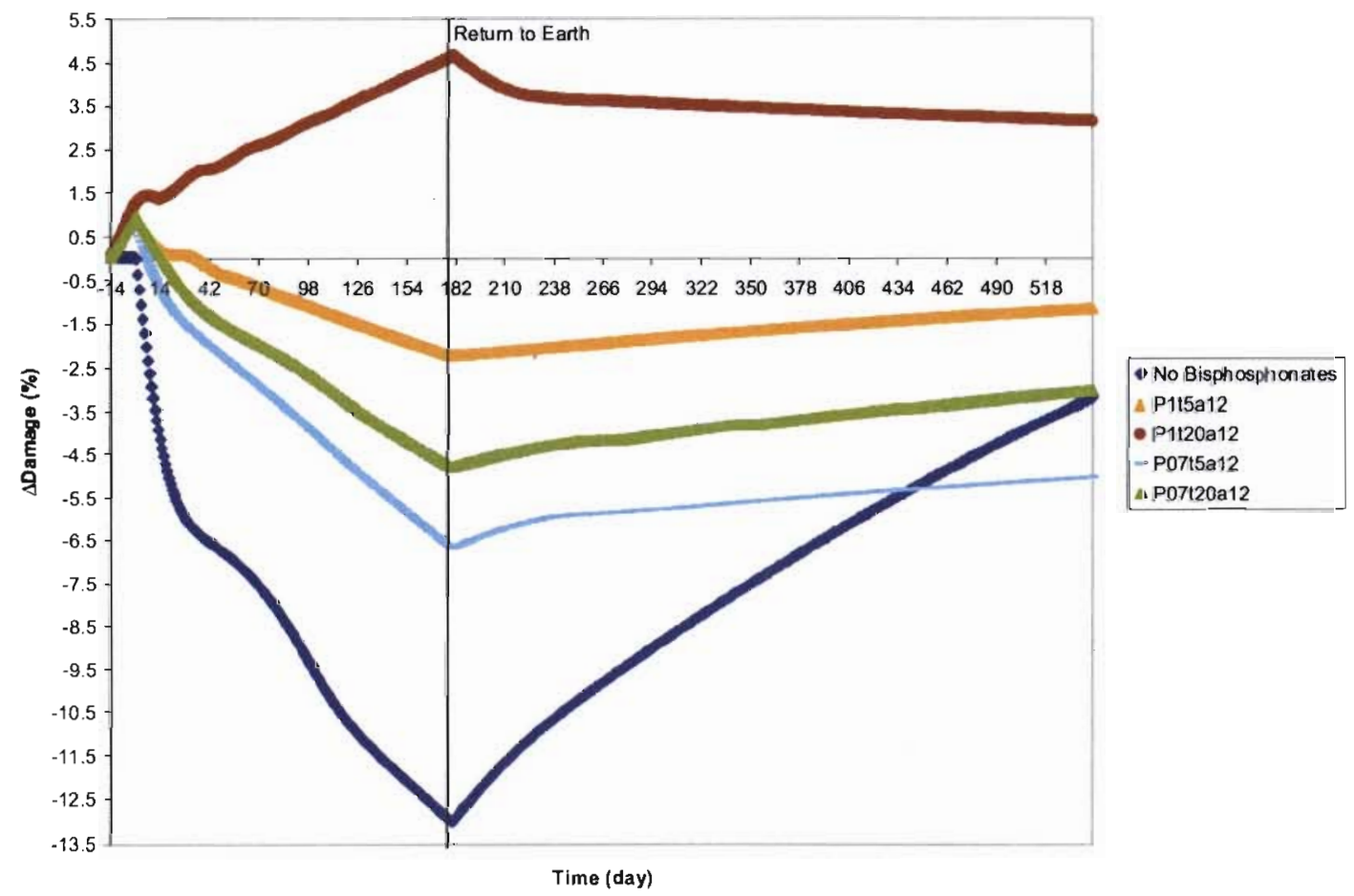

Figure D10. Predicted bisphosphonate effects beginning 14 days preflight on damage accumulation (D) and posttreatment return to Earth from 180-day spaceflight. 


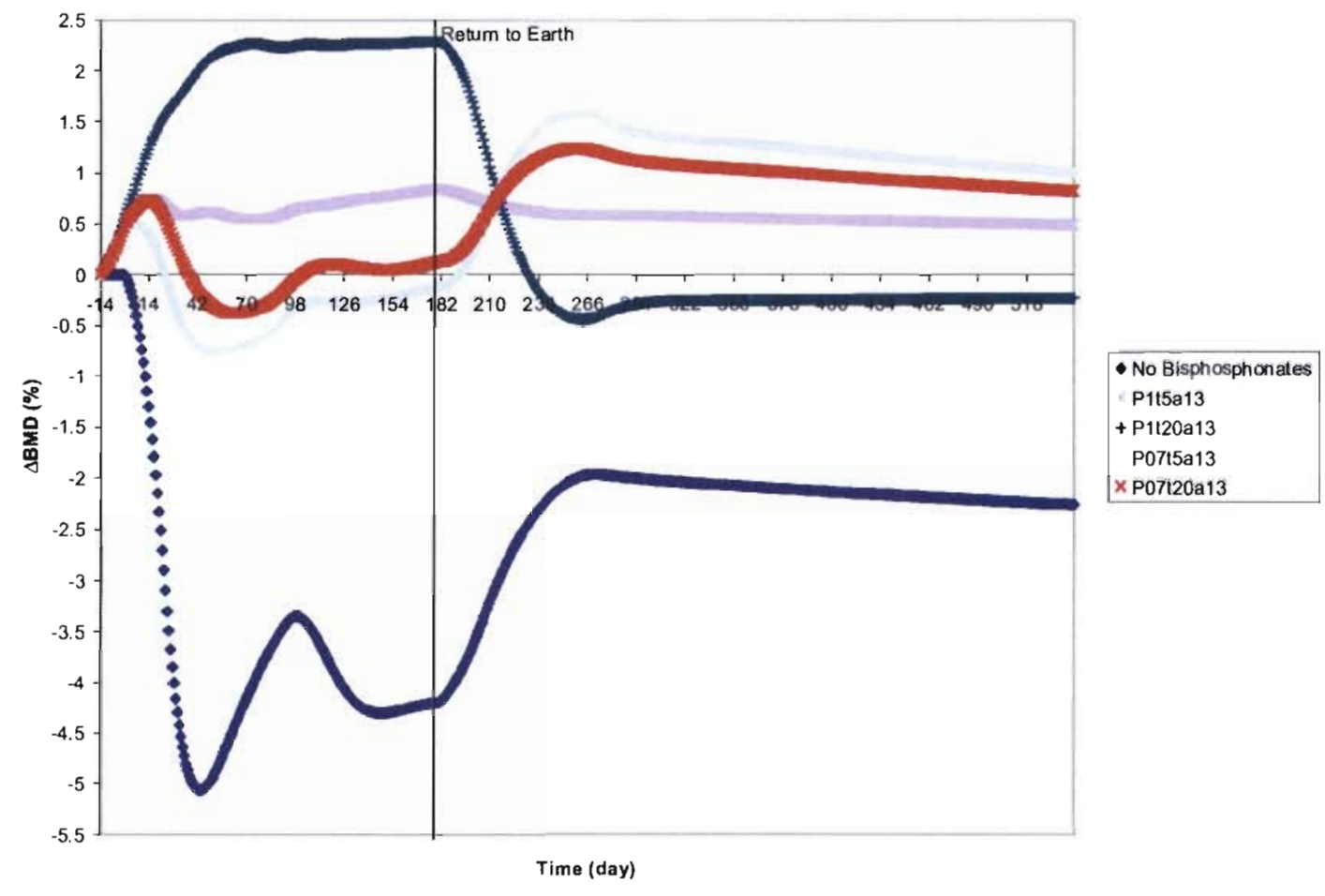

Figure D11. Predicted bisphosphonate effects beginning 14 days preflight on BMD and posttreatment return to Earth from 180-day spaceflight.

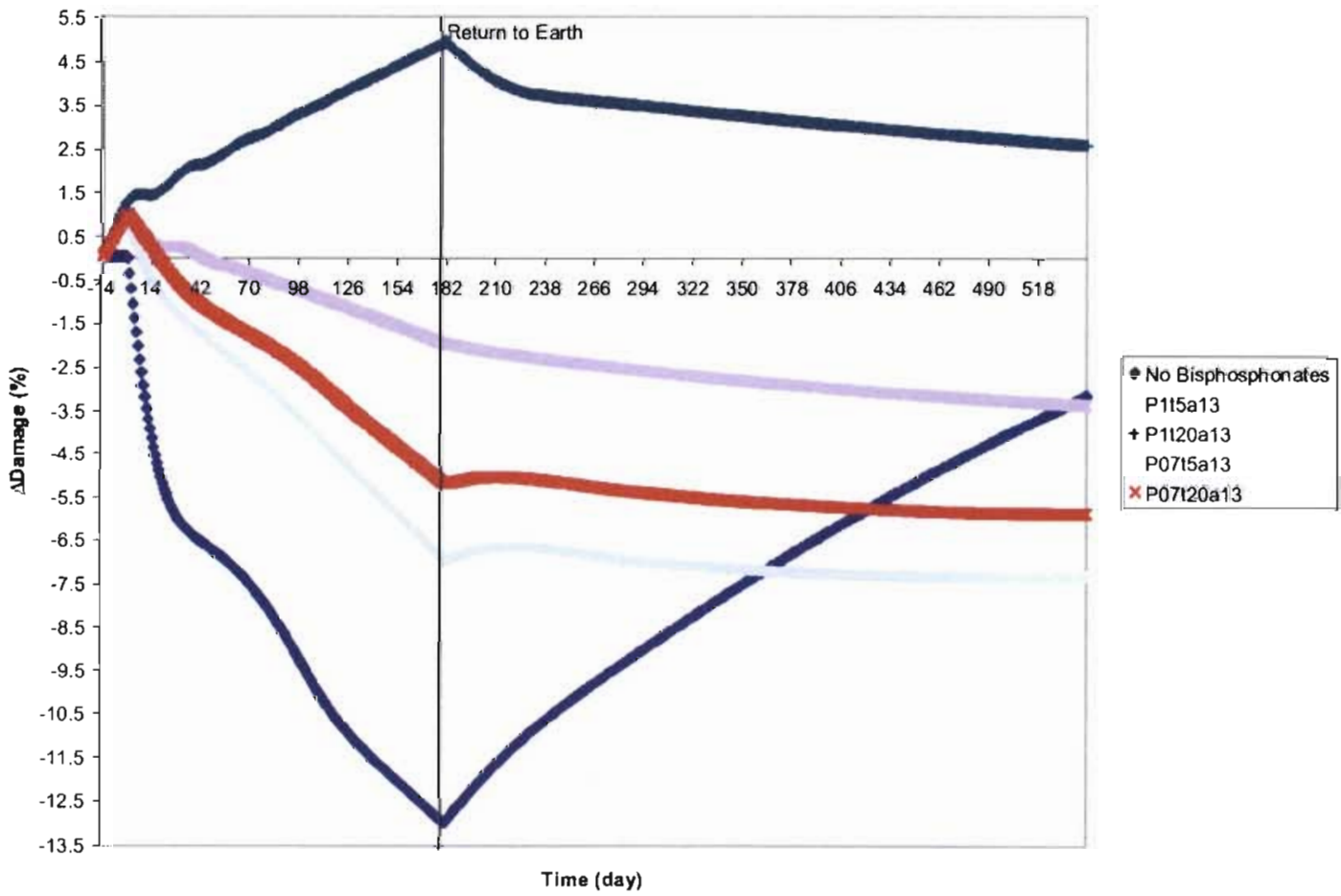

Figure D12. Predicted bisphosphonate effects beginning 14 days preflight on damage accumulation (D) and posttreatment return to Earth from 180-day spaceflight. 


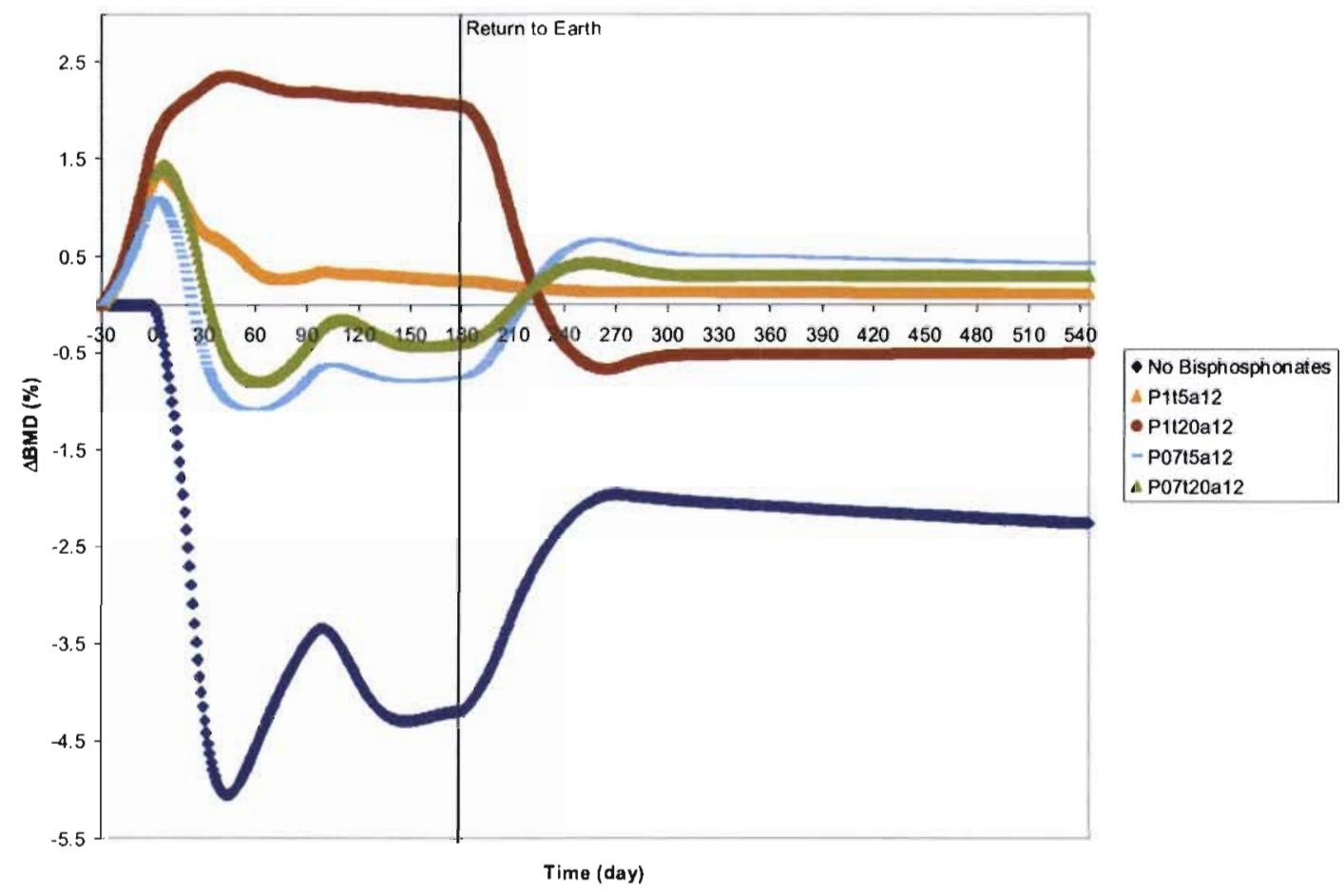

Figure D13. Predicted bisphosphonate effects beginning 30 days preflight on BMD and posttreatment return to Earth from 180-day spaceflight.

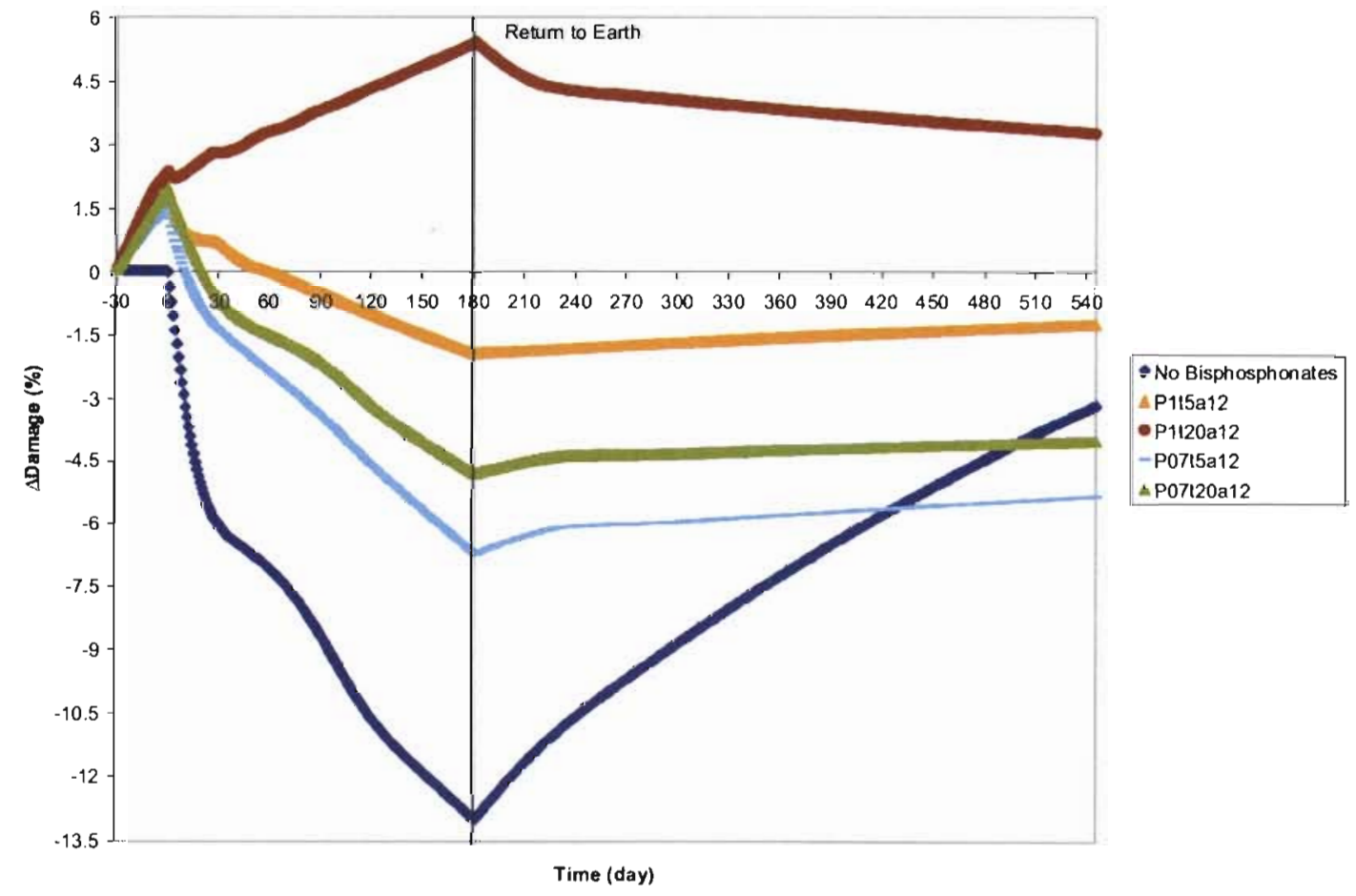

Figure D14. Predicted bisphosphonate effects beginning 30 days preflight on damage accumulation (D) and posttreatment return to Earth from 180-day spaceflight. 


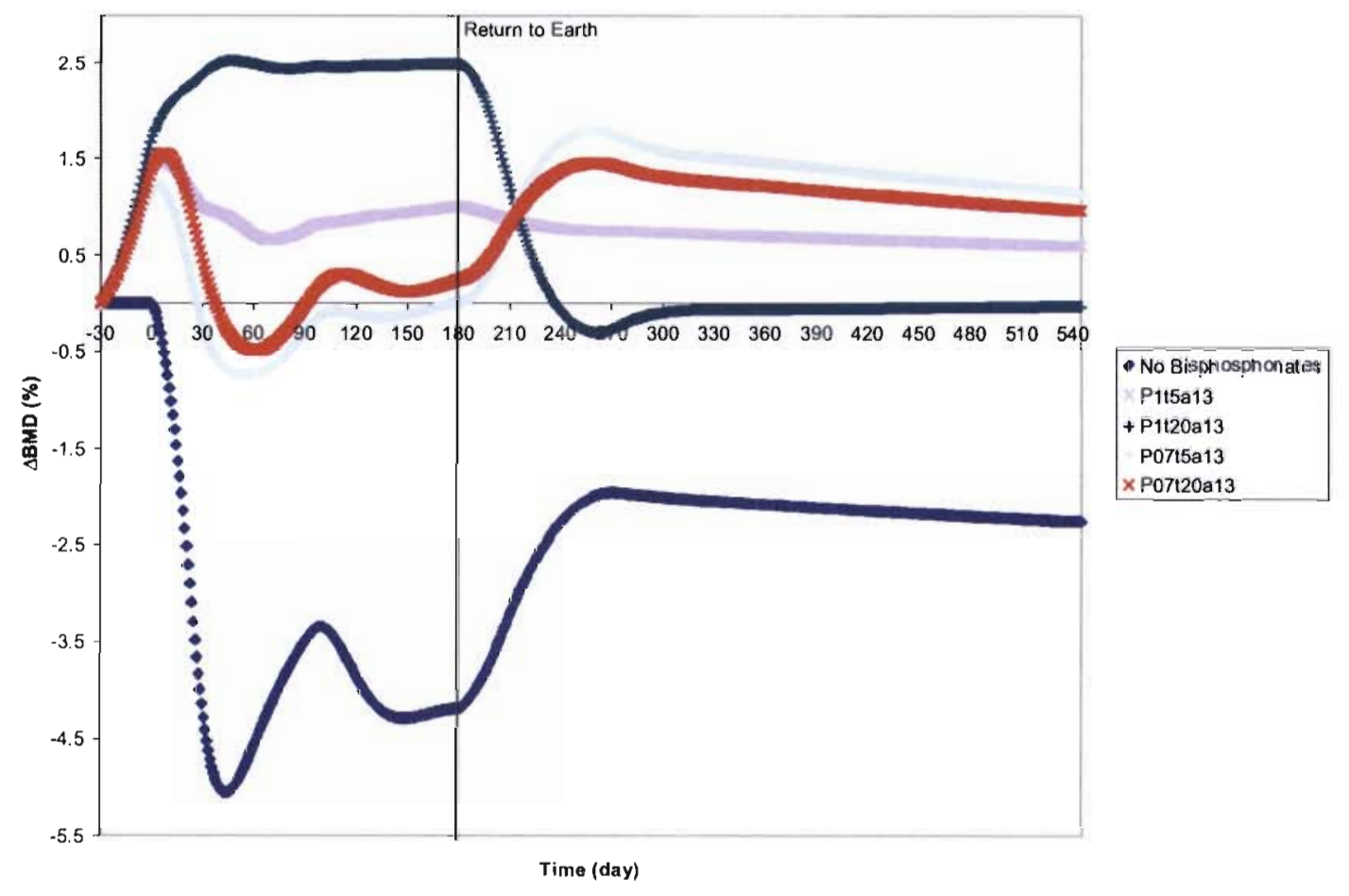

Figure D15. Predicted bisphosphonate effects beginning 30 days preflight on BMD and posttreatment return to Earth from 180 -day spaceflight.

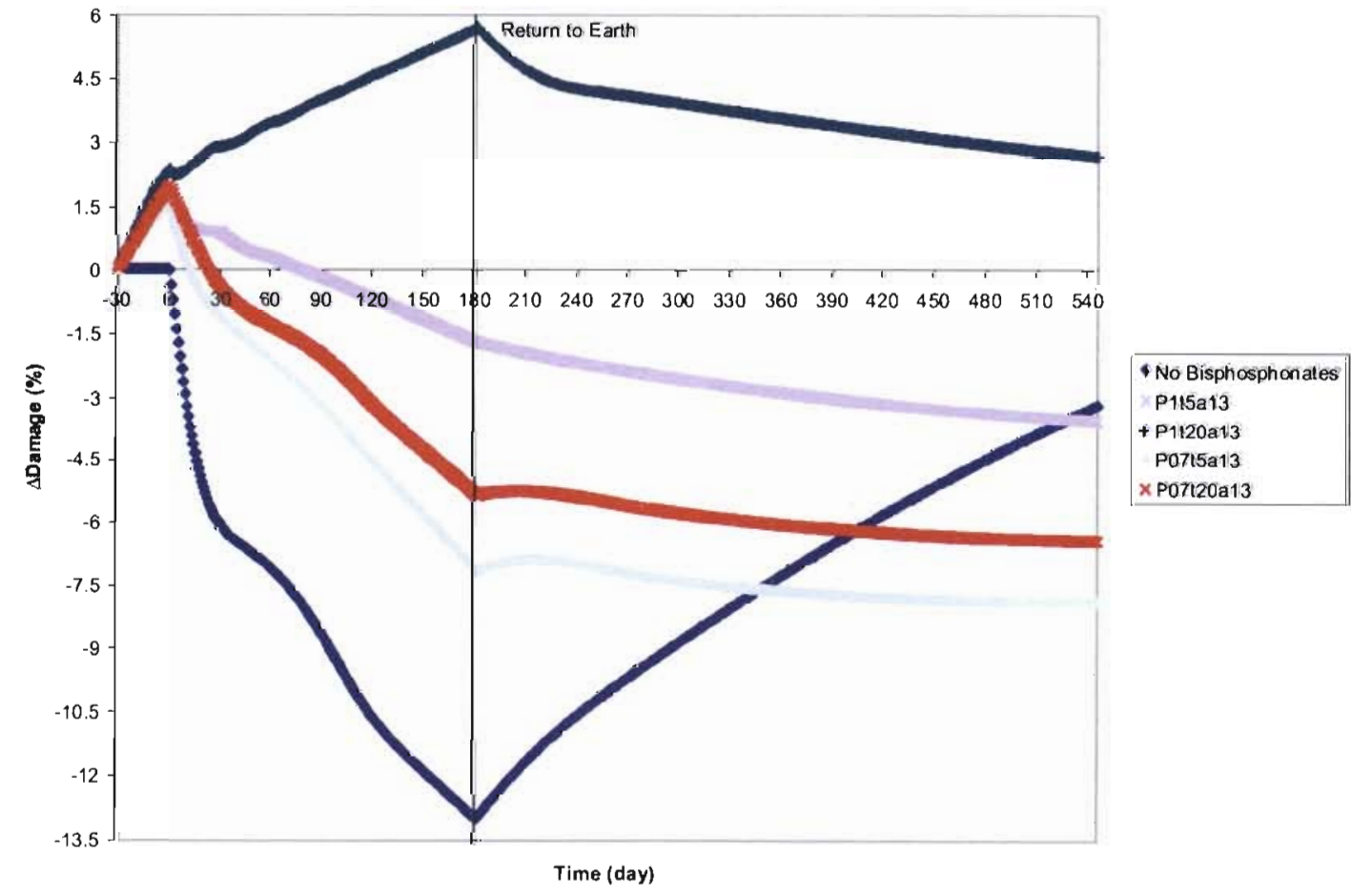

Figure D16. Predicted bisphosphonate effects beginning 30 days preflight on damage accumulation (D) and posttreatment return to Earth from 180-day spaceflight. 


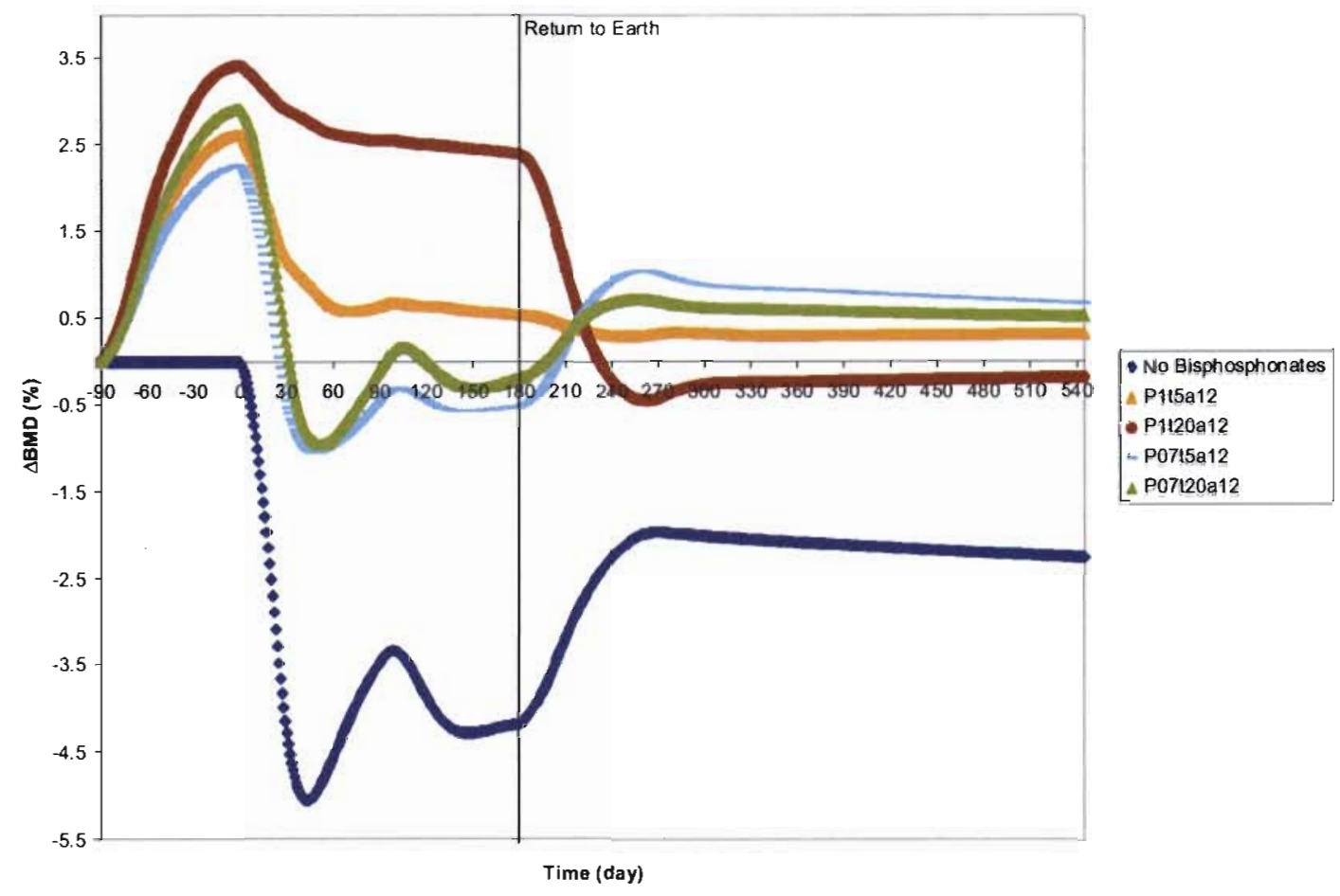

Figure D17. Predicted bisphosphonate effects beginning 90 days preflight on BMD and posttreatment return to Earth from 180-day spaceflight.

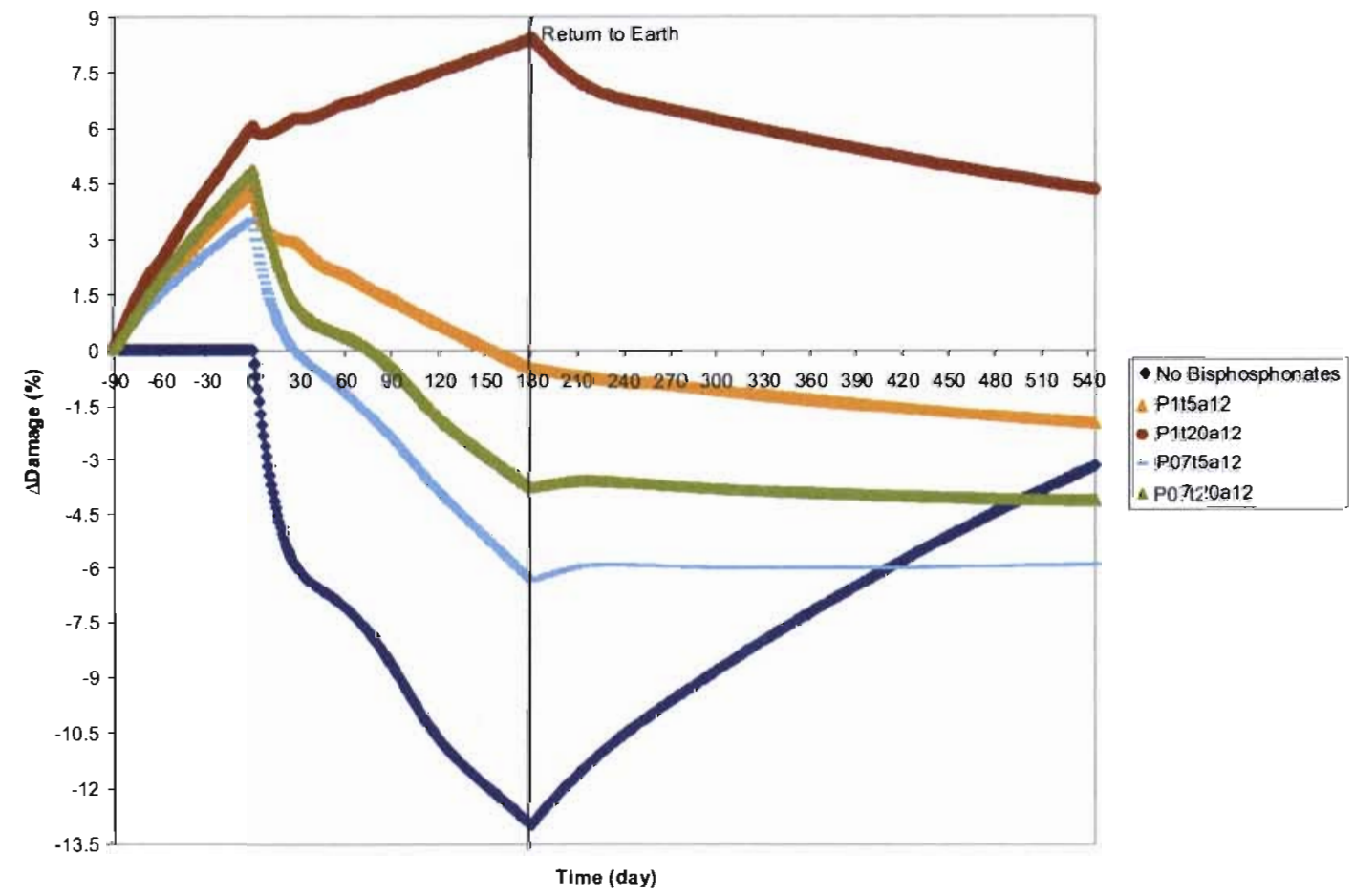

Figure D18. Predicted bisphosphonate effects beginning 90 days preflight on damage accumulation (D) and posttreatment return to Earth from 180-day spaceflight. 


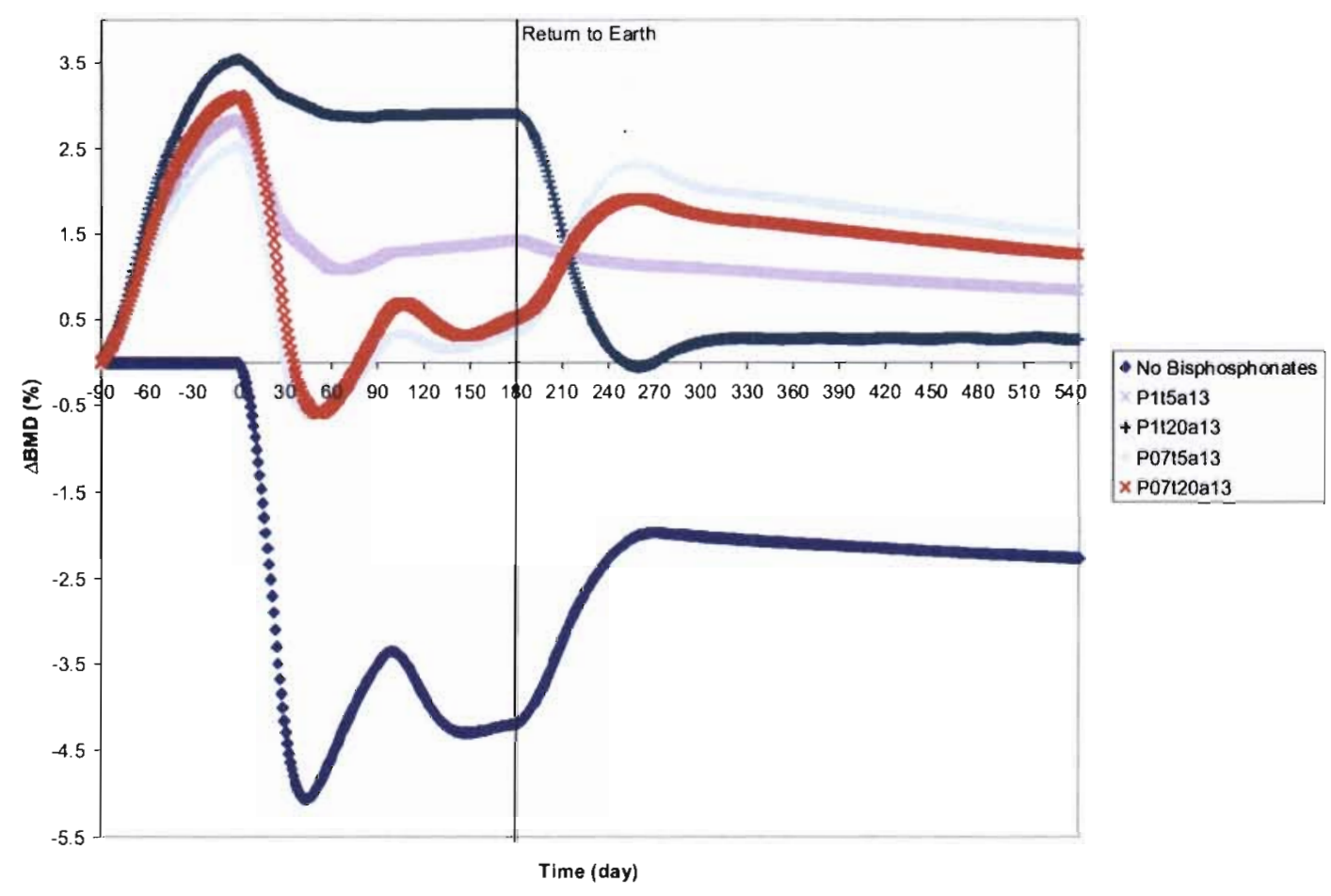

Figure D19. Predicted bisphosphonate effects beginning 90 days preflight on BMD and posttreatment return to Earth from 180-day spaceflight.

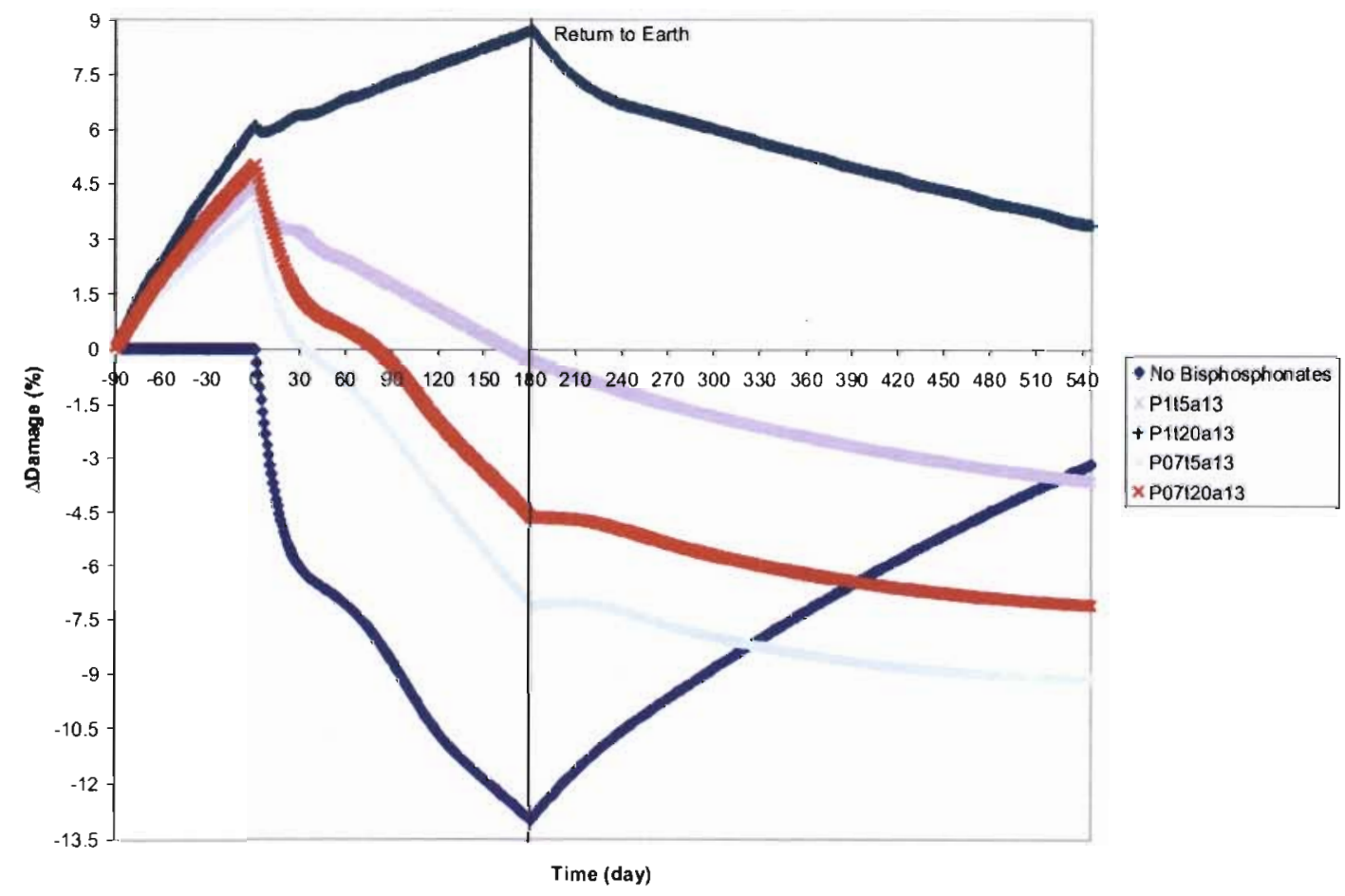

Figure D20. Predicted bisphosphonate effects beginning 90 days preflight on damage accumulation (D) and posttreatment return to Earth from 180-day spaceflight. 


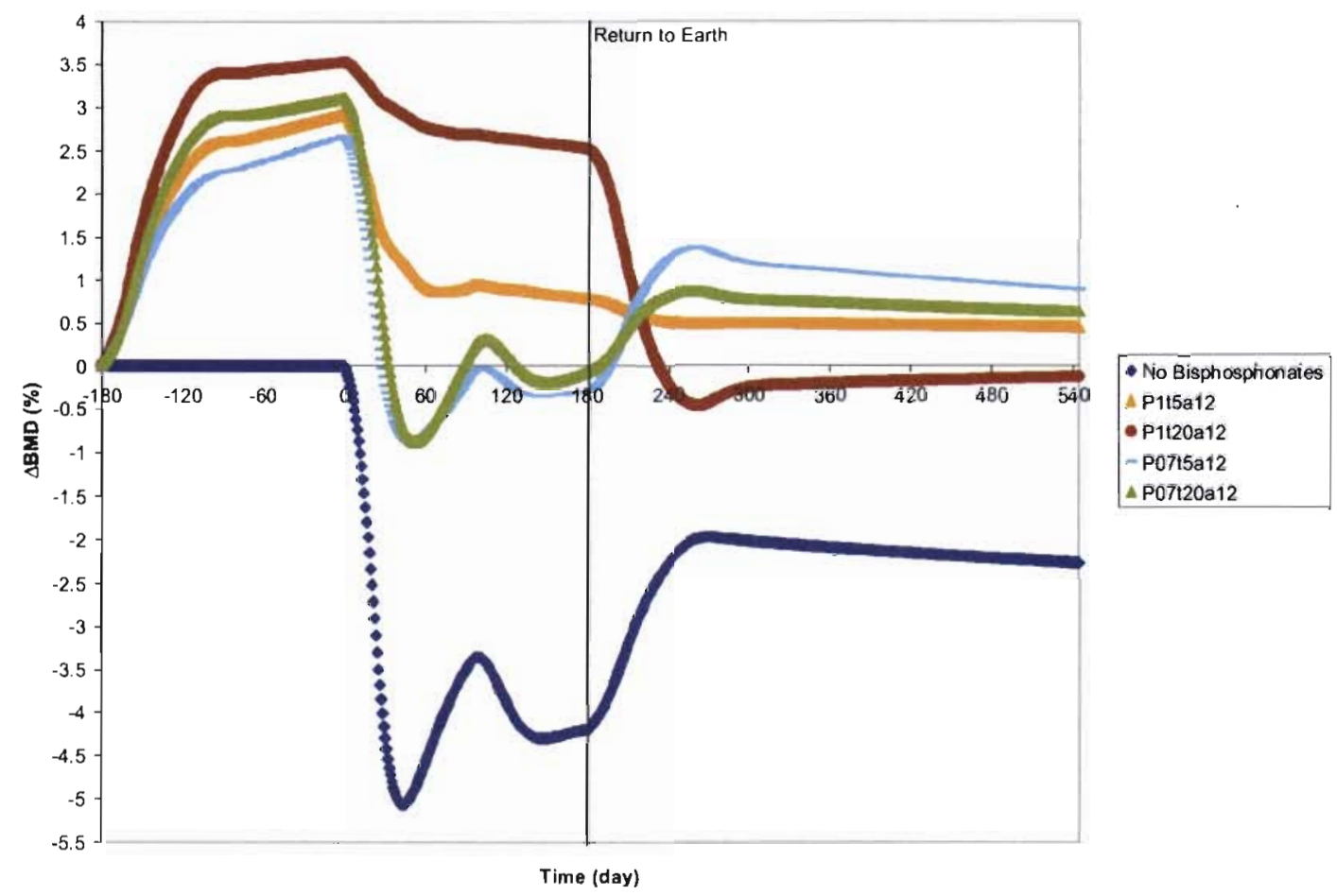

Figure D21. Predicted bisphosphonate effects beginning 180 days preflight on BMD and posttreatment return to Earth from 180-day spaceflight.

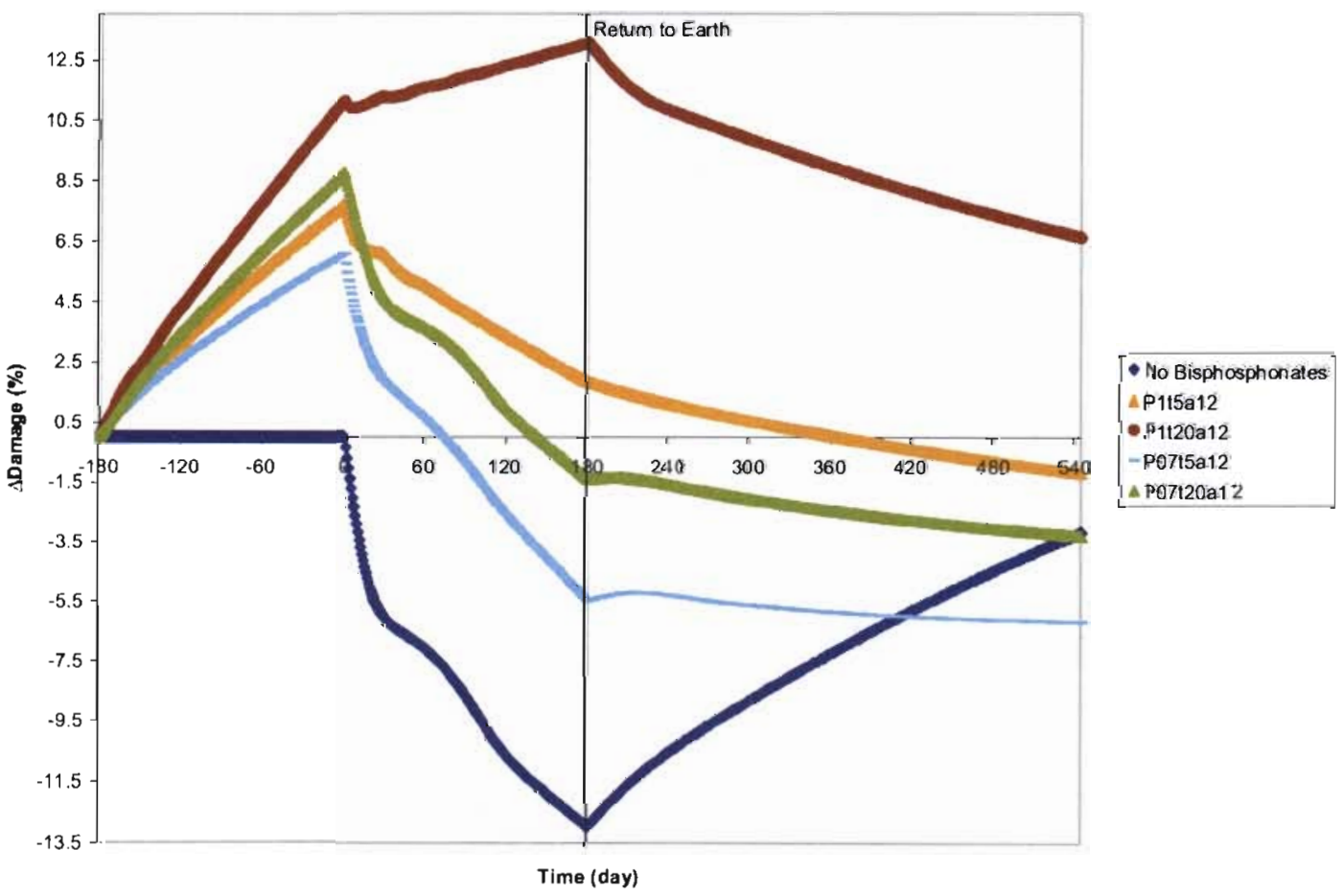

Figure D22. Predicted bisphosphonate effects beginning 180 days preflight on damage accumulation (D) and posttreatment return to Earth from 180-day spaceflight. 


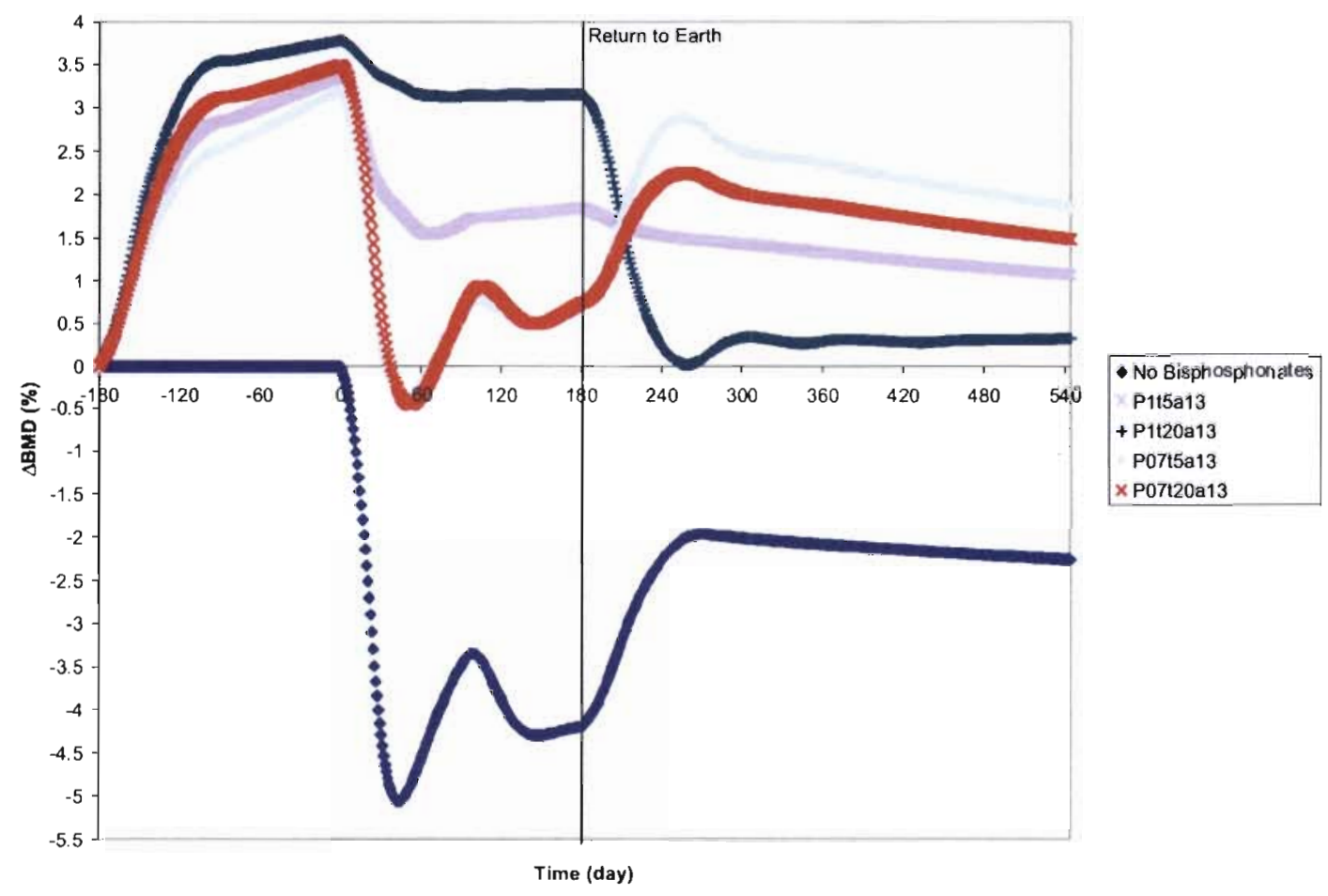

Figure D23. Predicted bisphosphonate effects beginning 180 days preflight on BMD and posttreatment return to Earth from 180-day spaceflight.

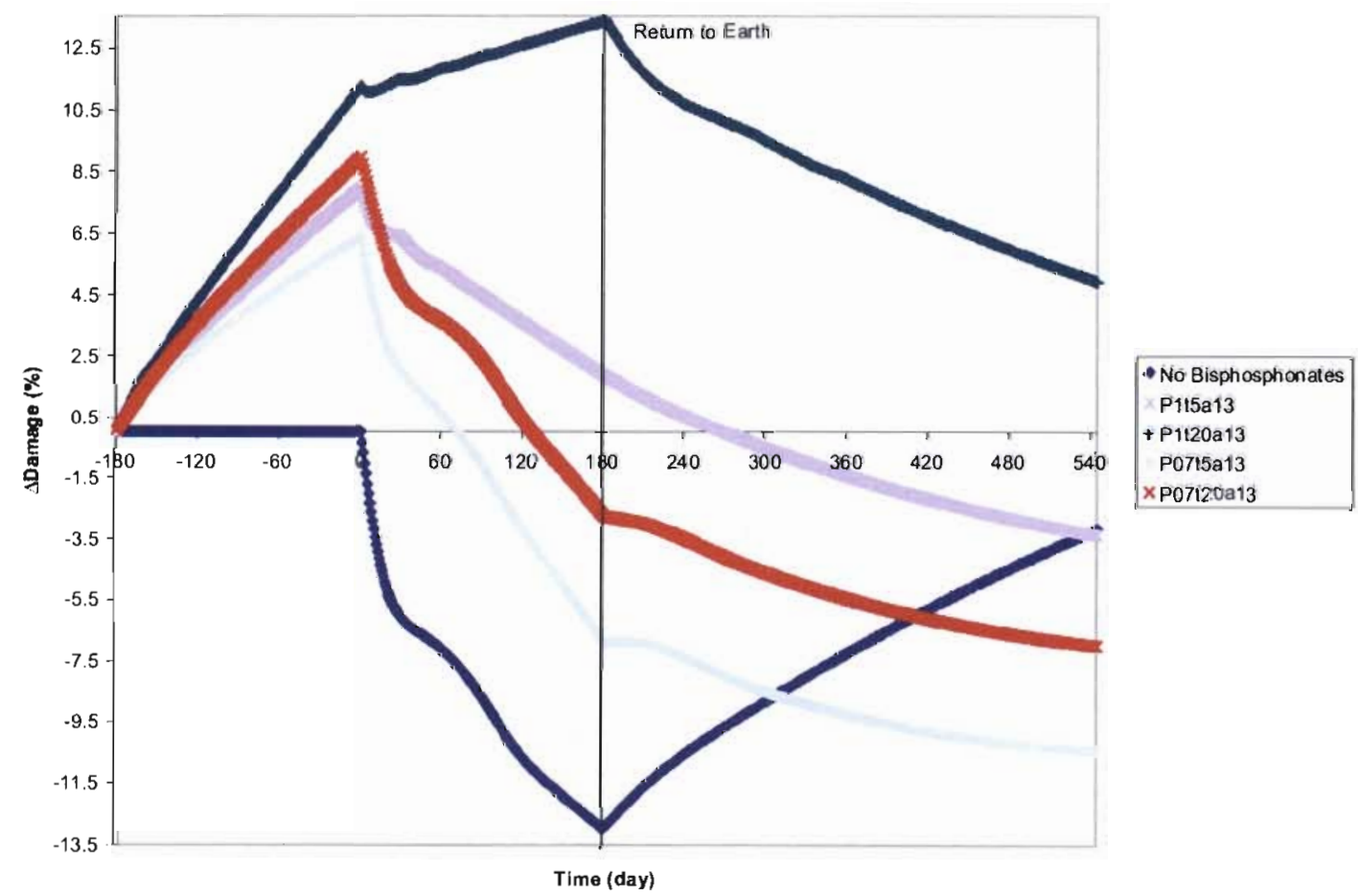

Figure D24. Predicted bisphosphonate effects beginning 180 days preflight on damage accumulation (D) and posttreatment return to Earth from 180-day spaceflight. 


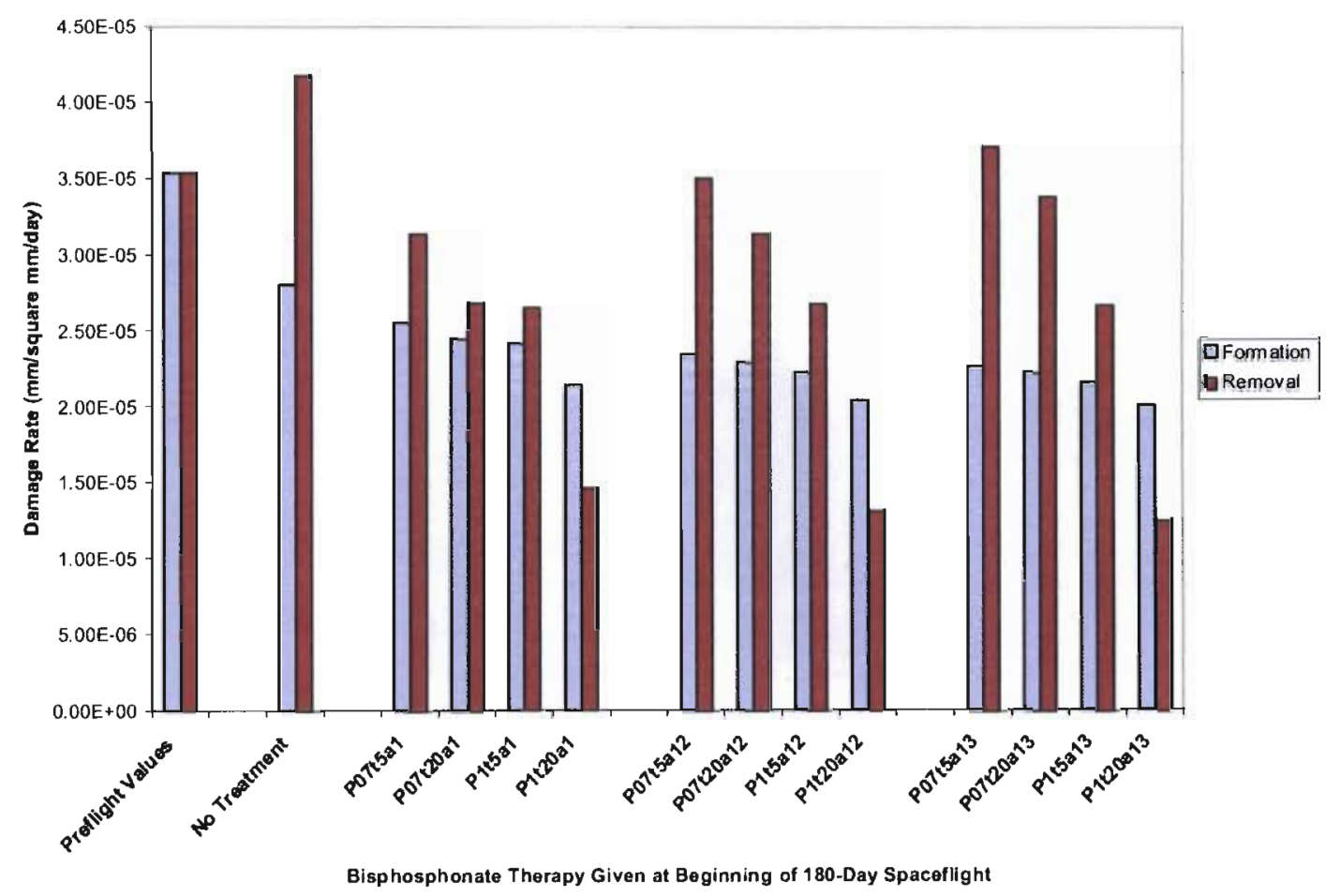

Figure D25. Predicted effects of 180-day spaceflight with and without bisphosphonate treatment on damage formation and removal rates. Note that these are end-of-flight values. 


\section{APPENDIX E: FIGURES (365-DAY SPACEFLIGHT)}

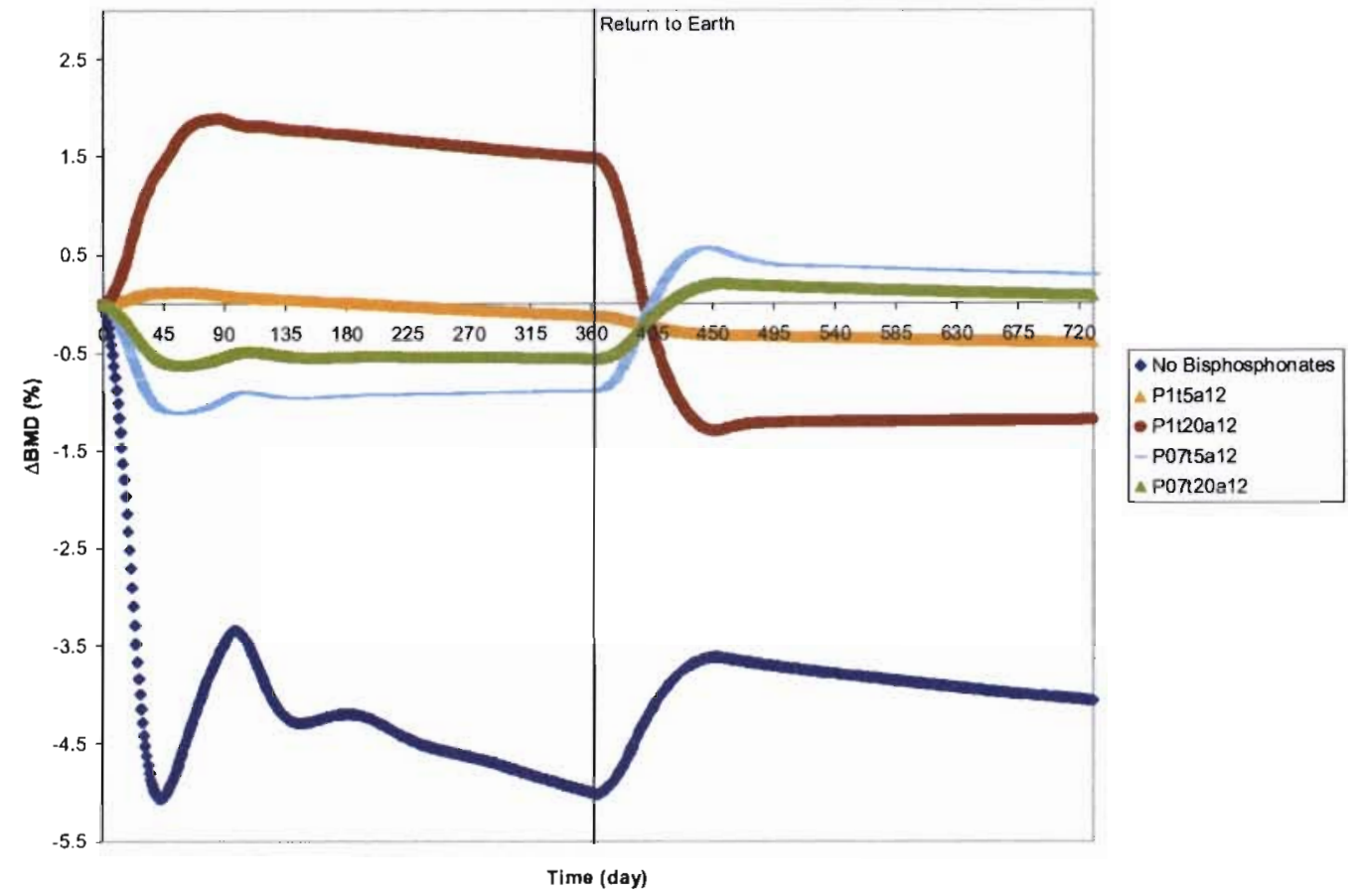

Figure E1. Predicted bisphosphonate effects on BMD and posttreatment return to Earth from 365day spaceflight.

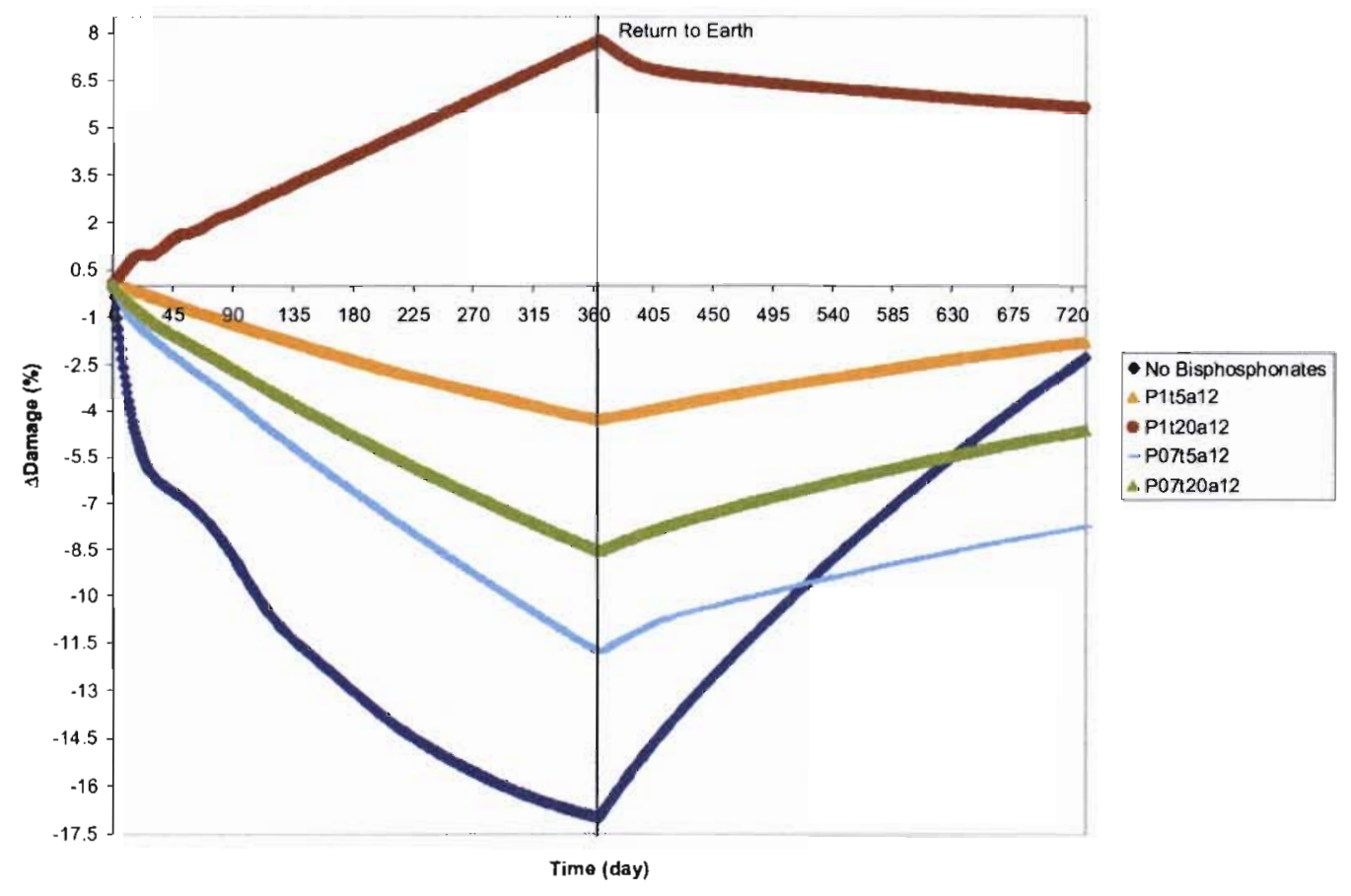

Figure E2. Predicted bisphosphonate effects on damage accumulation (D) and posttreatment return to Earth from 365-day spaceflight. 


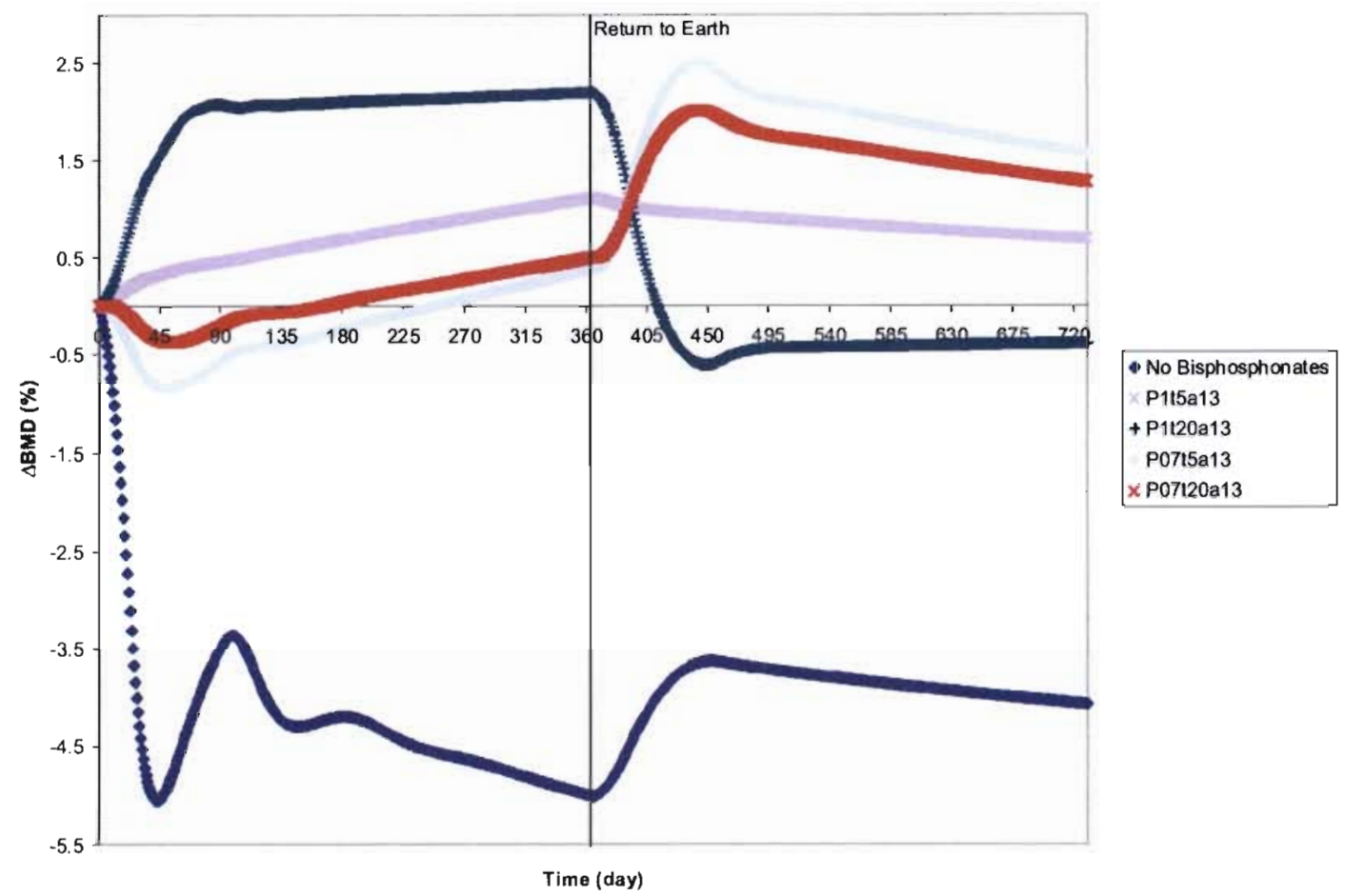

Figure E3. Predicted bisphosphonate effects on BMD and posttreatment return to Earth from 365day spaceflight.

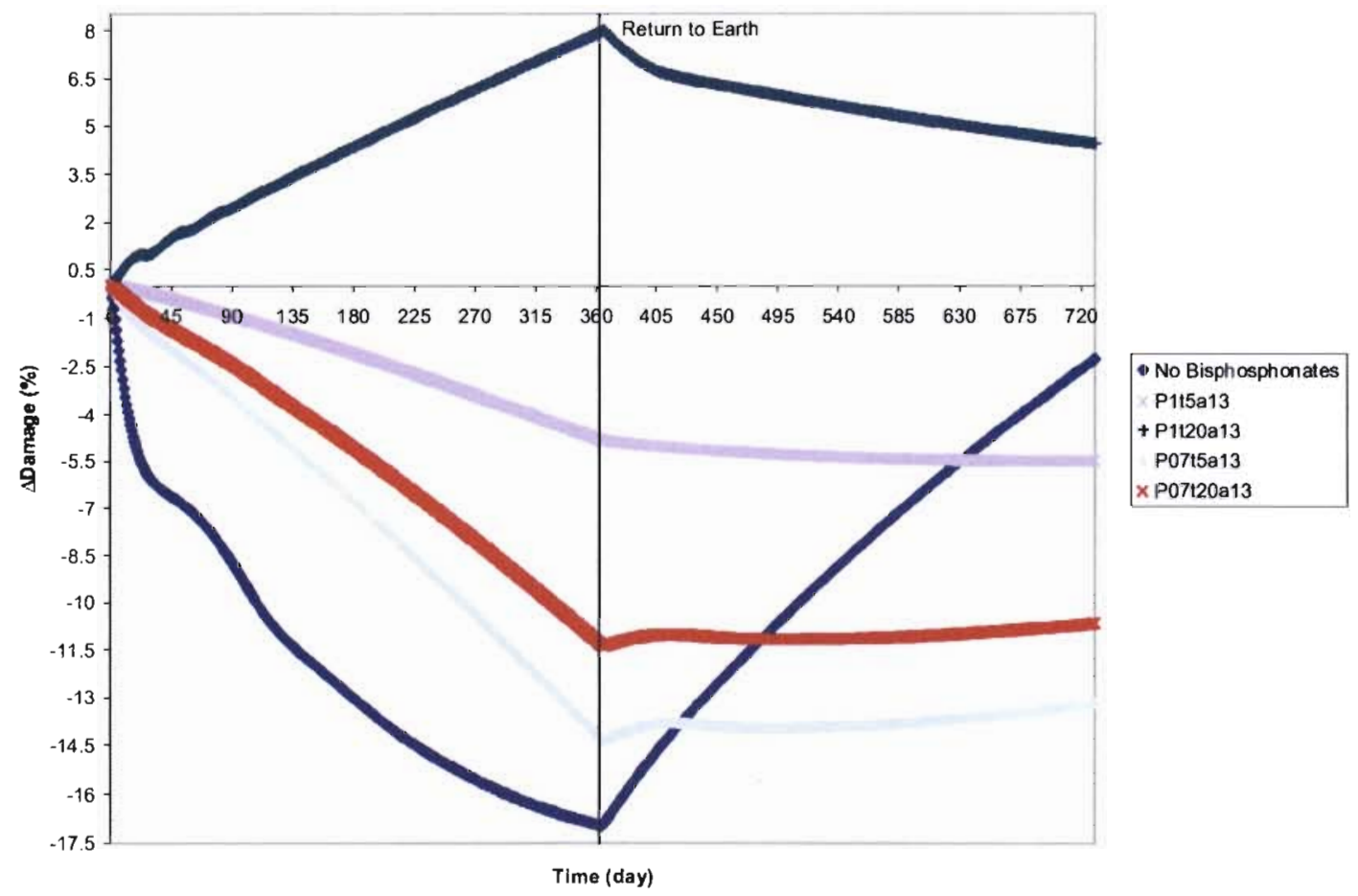

Figure E4. Predicted bisphosphonate effects on damage accumulation (D) and posttreatment return to Earth from 365-day spaceflight. 


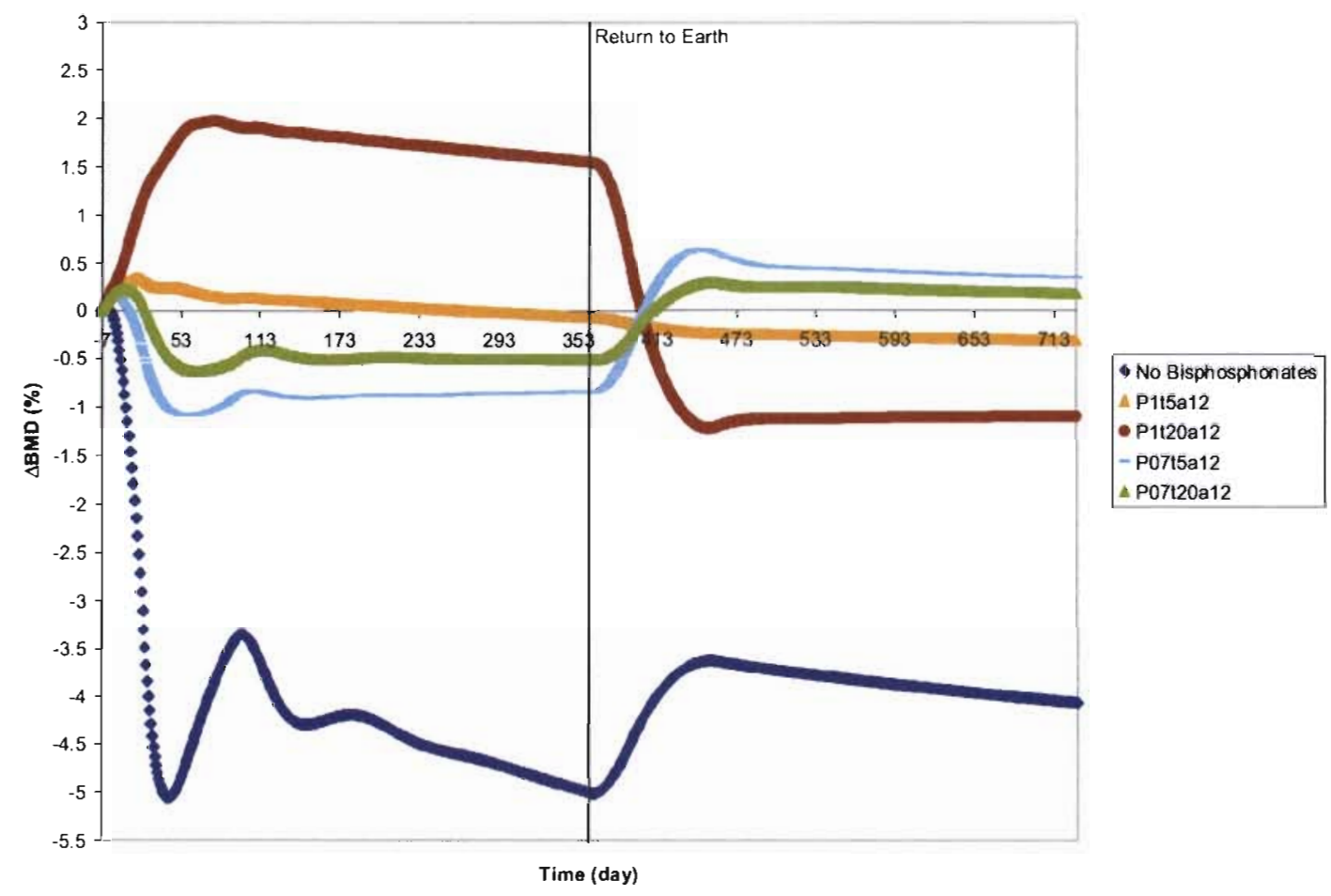

Figure E5. Predicted bisphosphonate effects beginning 7 days preflight on BMD and posttreatment return to Earth from 365-day spaceflight.

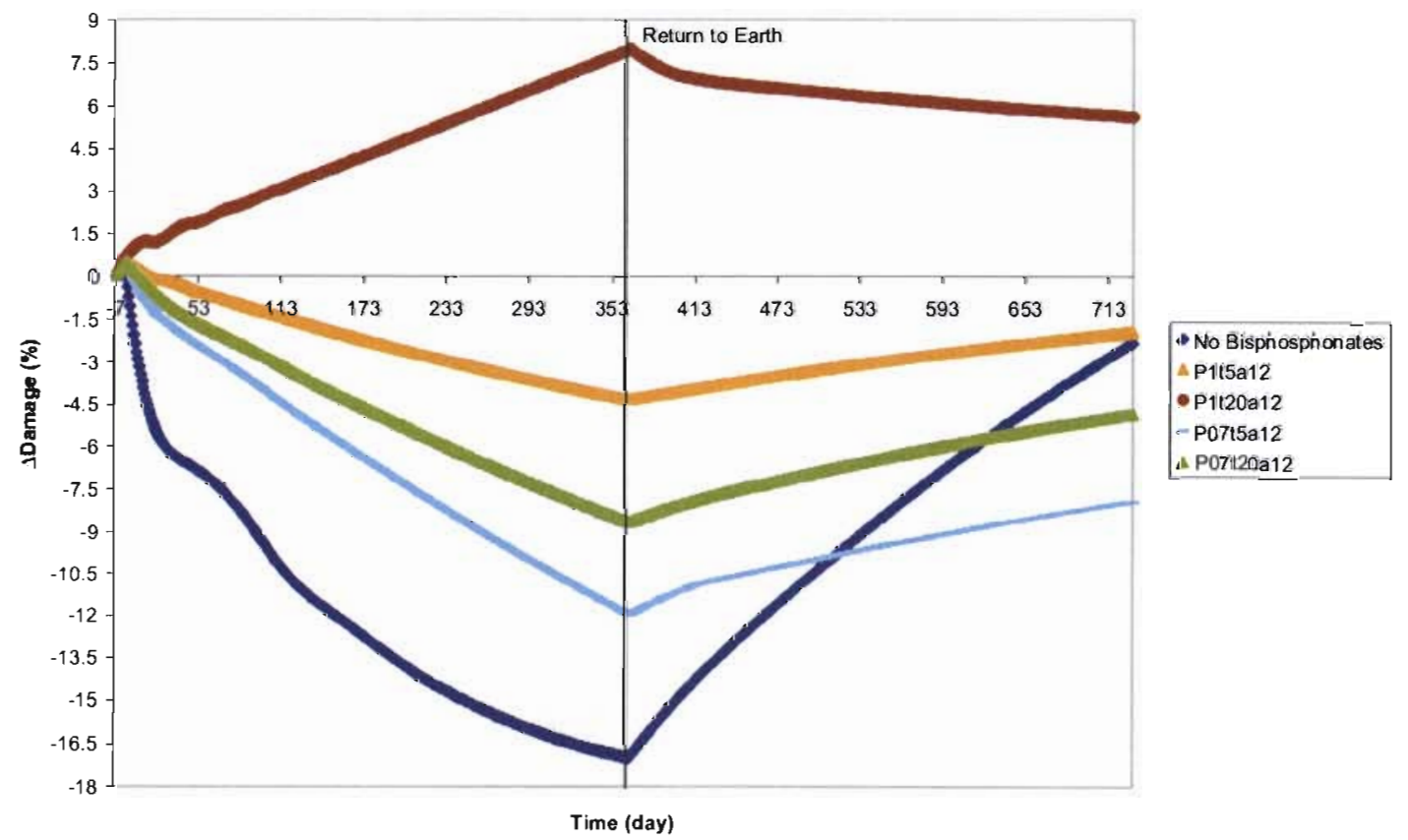

Figure E6. Predicted bisphosphonate effects beginning 7 days preflight on damage accumulation (D) and posttreatment return to Earth from 365-day spaceflight. 


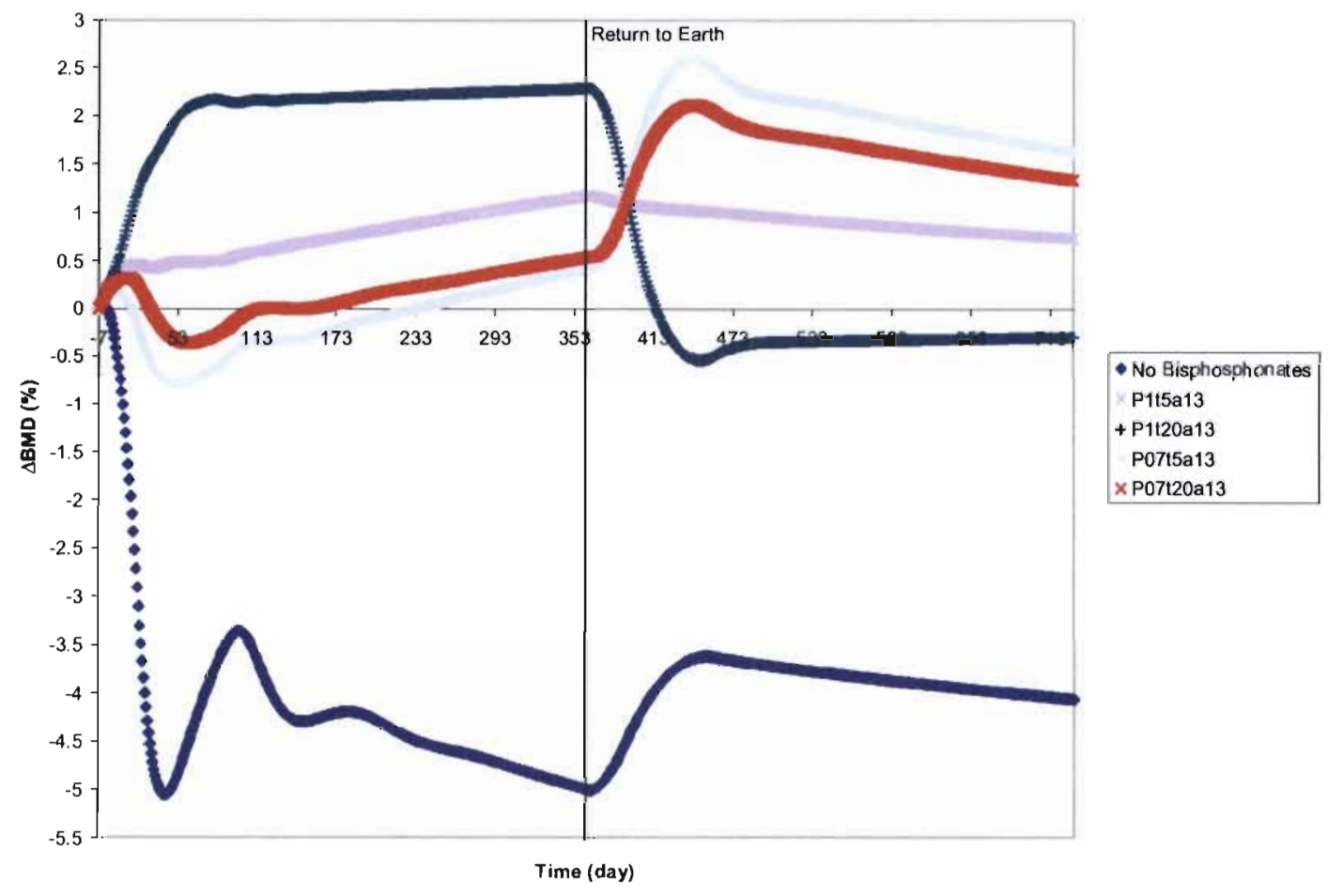

Figure E7. Predicted bisphosphonate effects beginning 7 days preflight on BMD and posttreatment return to Earth from 365-day spaceflight.

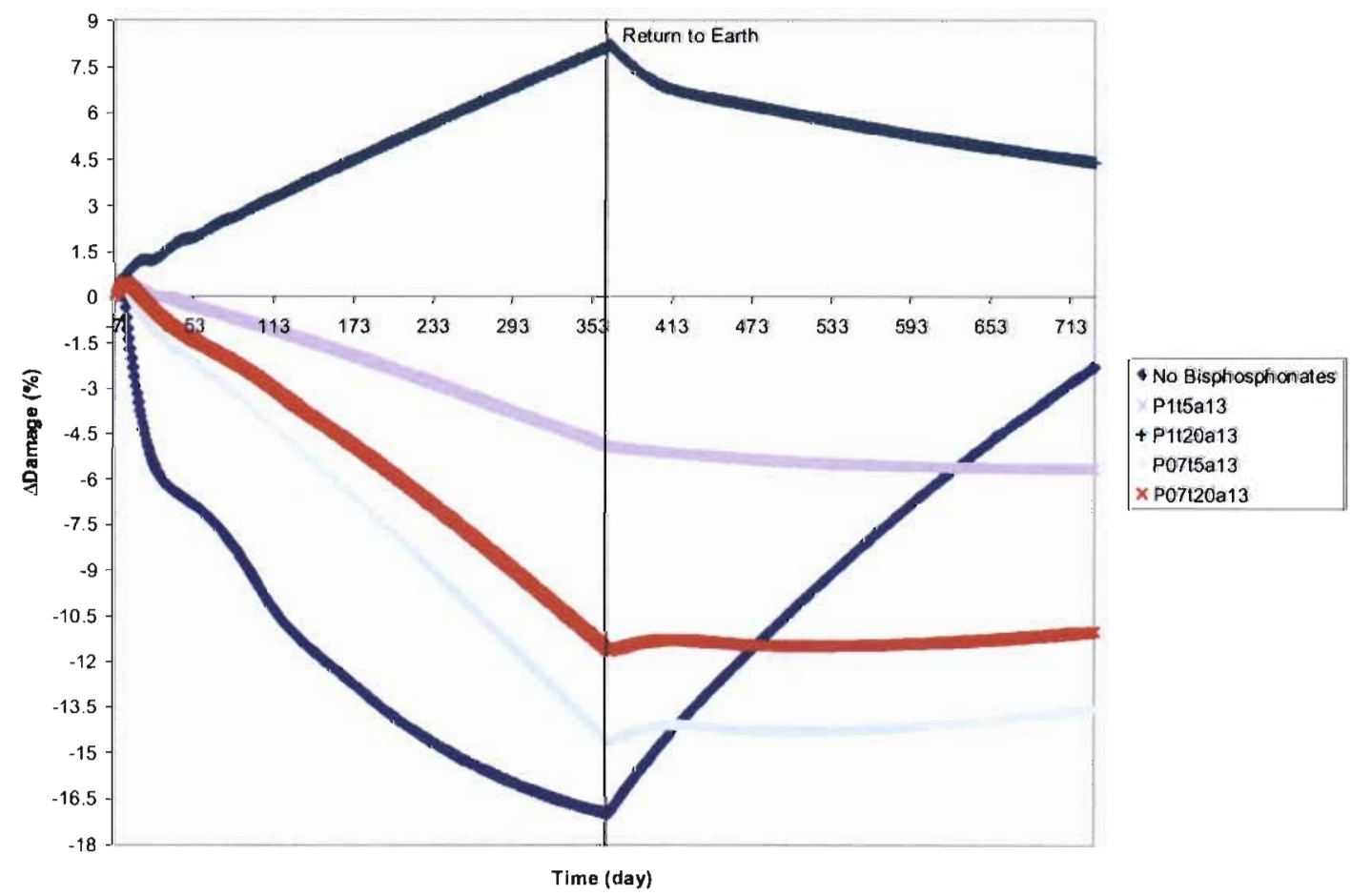

Figure E8. Predicted bisphosphonate effects beginning 7 days preflight on damage accumulation (D) and posttreatment return to Earth from 365-day spaceflight. 


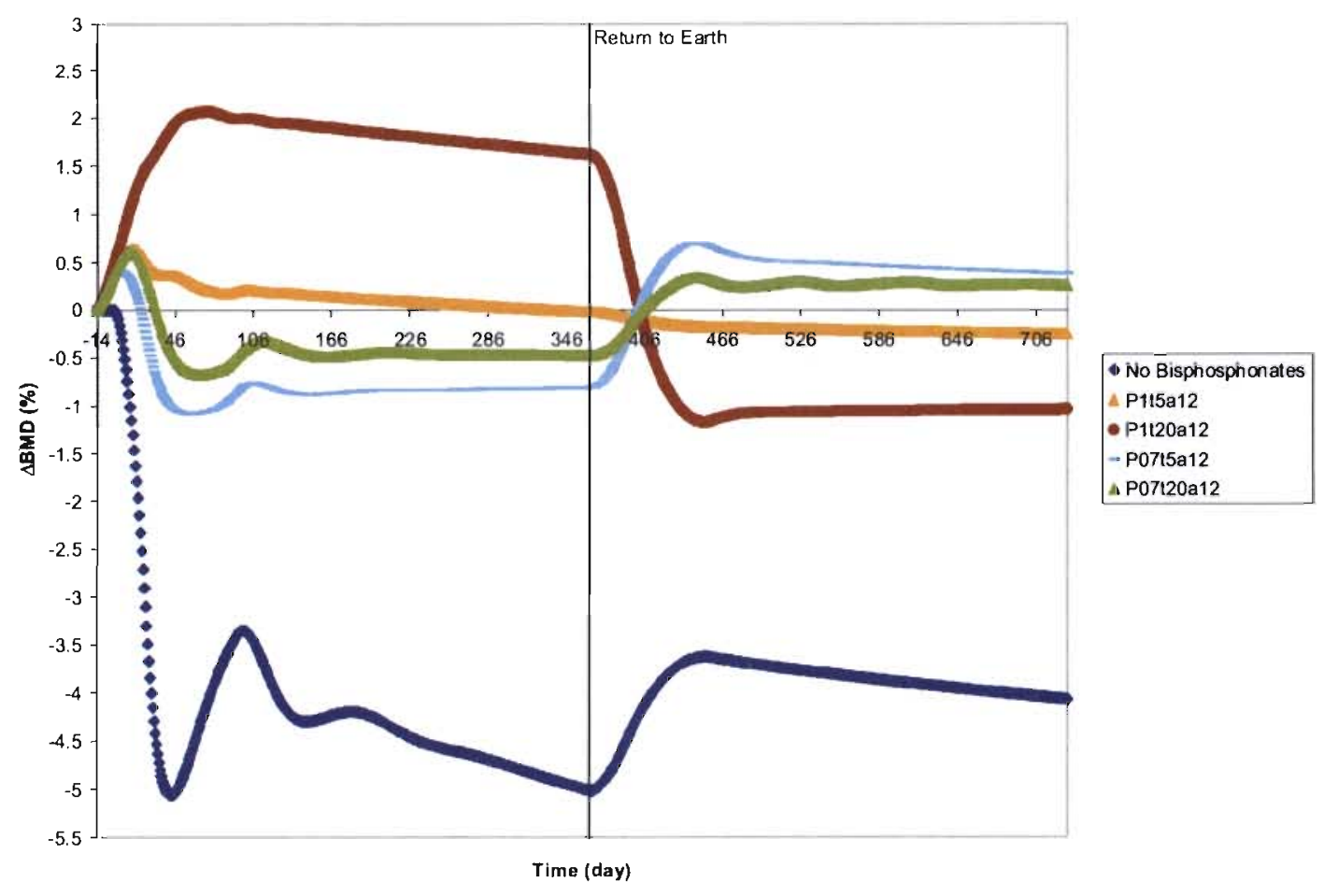

Figure E9. Predicted bisphosphonate effects beginning 14 days preflight on BMD and posttreatment return to Earth from 365 -day spaceflight.

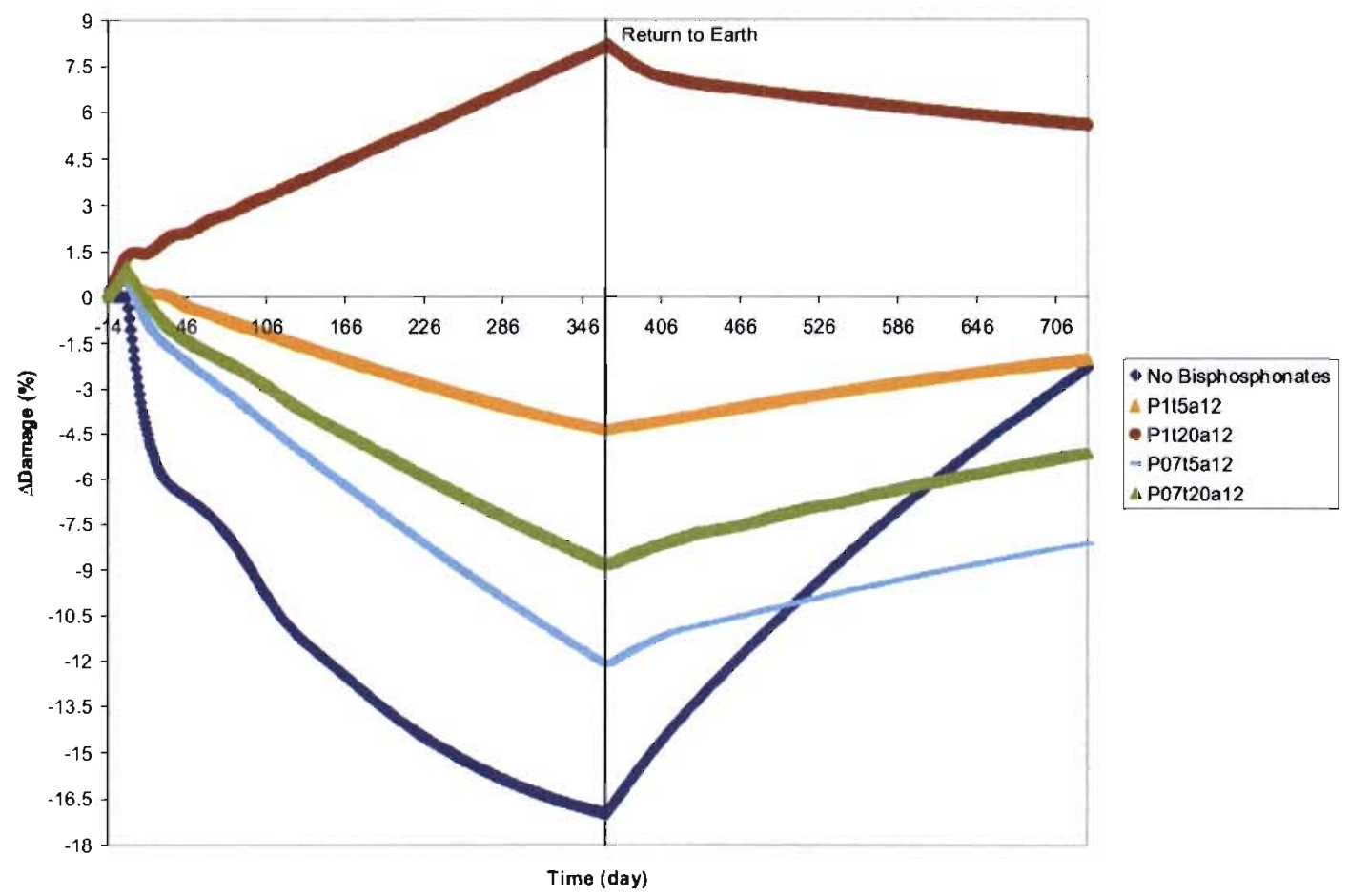

Figure E10. Predicted bisphosphonate effects beginning 14 days preflight on damage accumulation (D) and posttreatment return to Earth from 365-day spaceflight. 


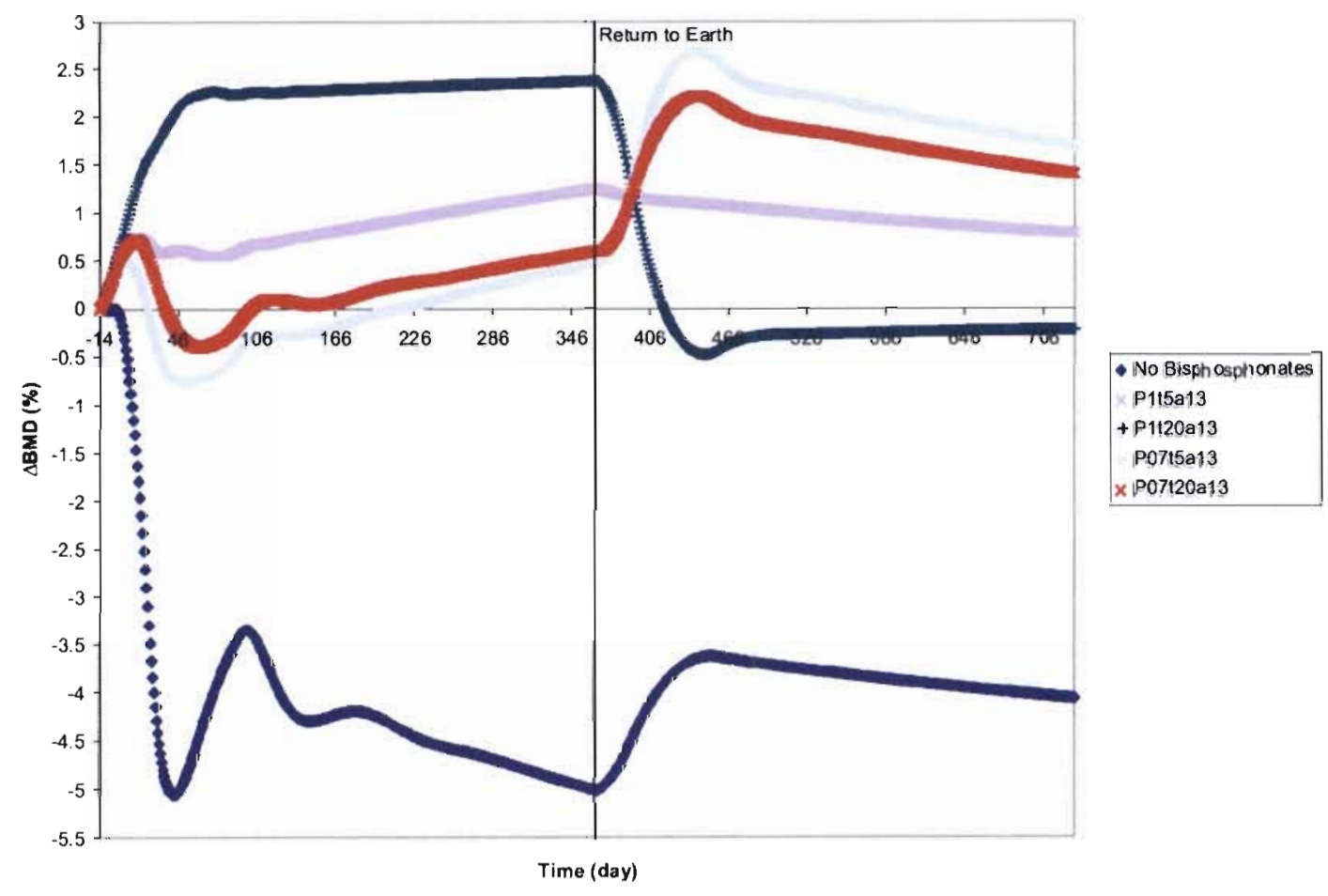

Figure E11. Predicted bisphosphonate effects beginning 14 days preflight on BMD and posttreatment return to Earth from 365-day spaceflight.

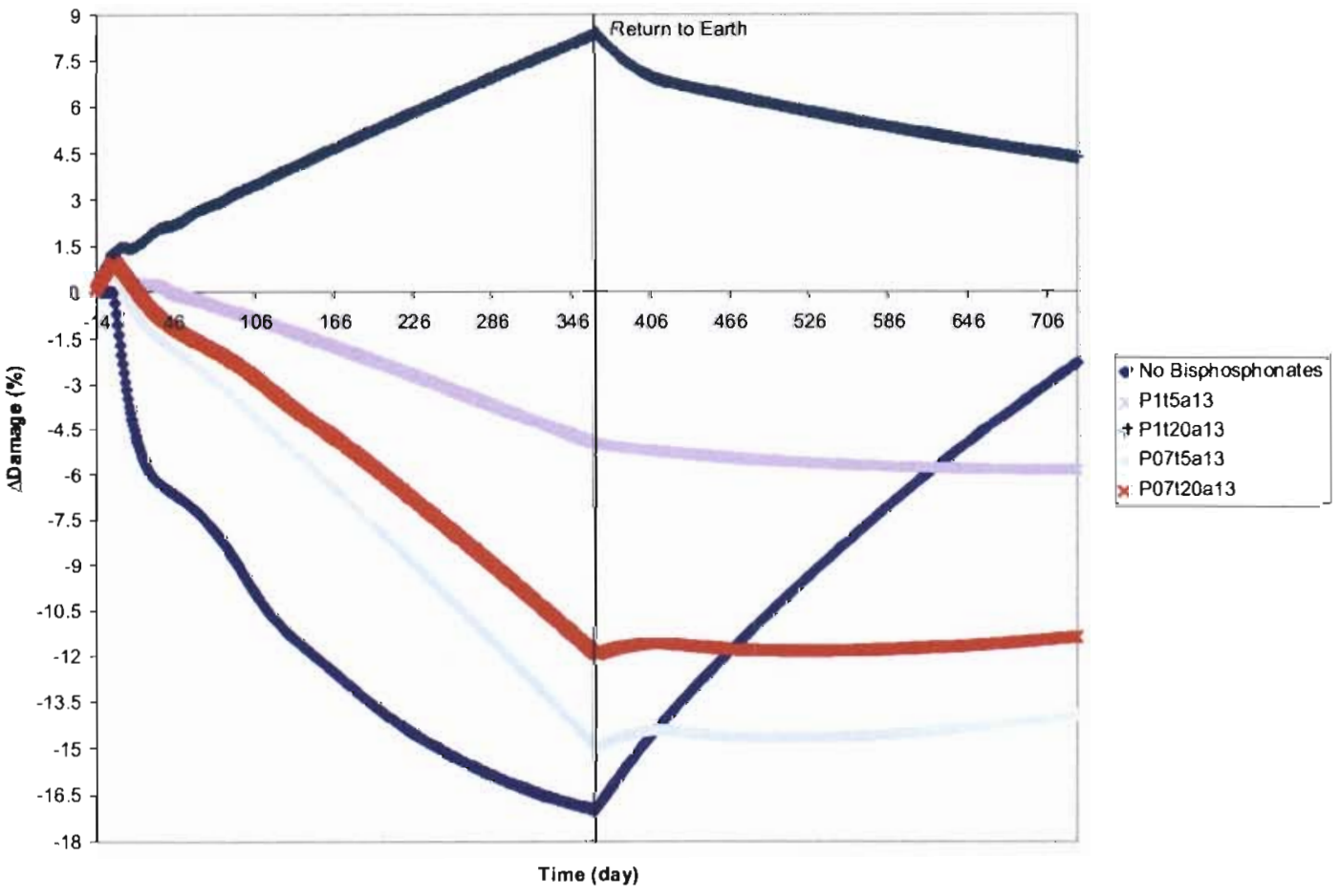

Figure E12. Predicted bisphosphonate effects beginning 14 days preflight on damage accumulation (D) and posttreatment return to Earth from 365-day spaceflight. 


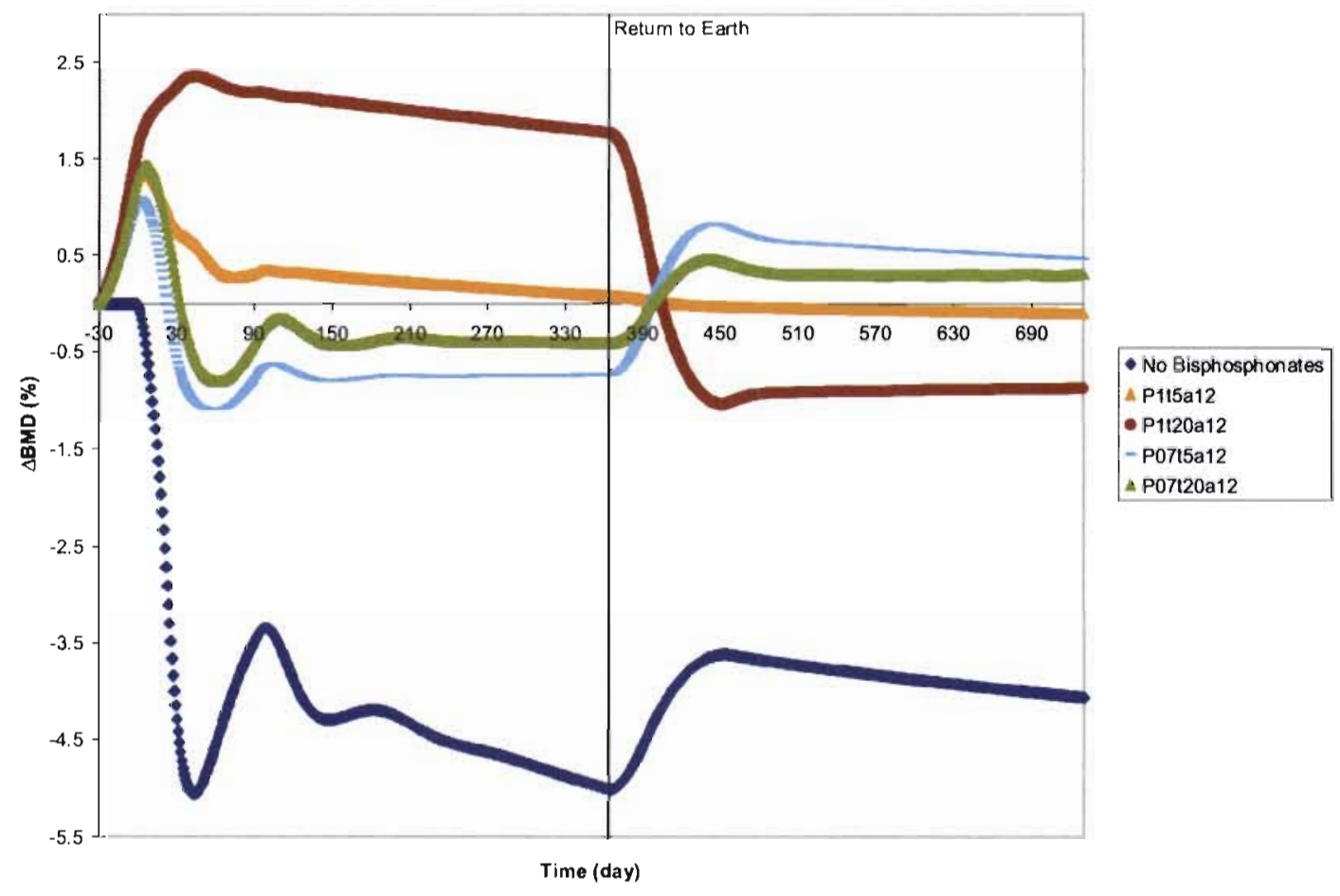

Figure E13. Predicted bisphosphonate effects beginning 30 days preflight on BMD and posttreatment return to Earth from 365-day spaceflight.

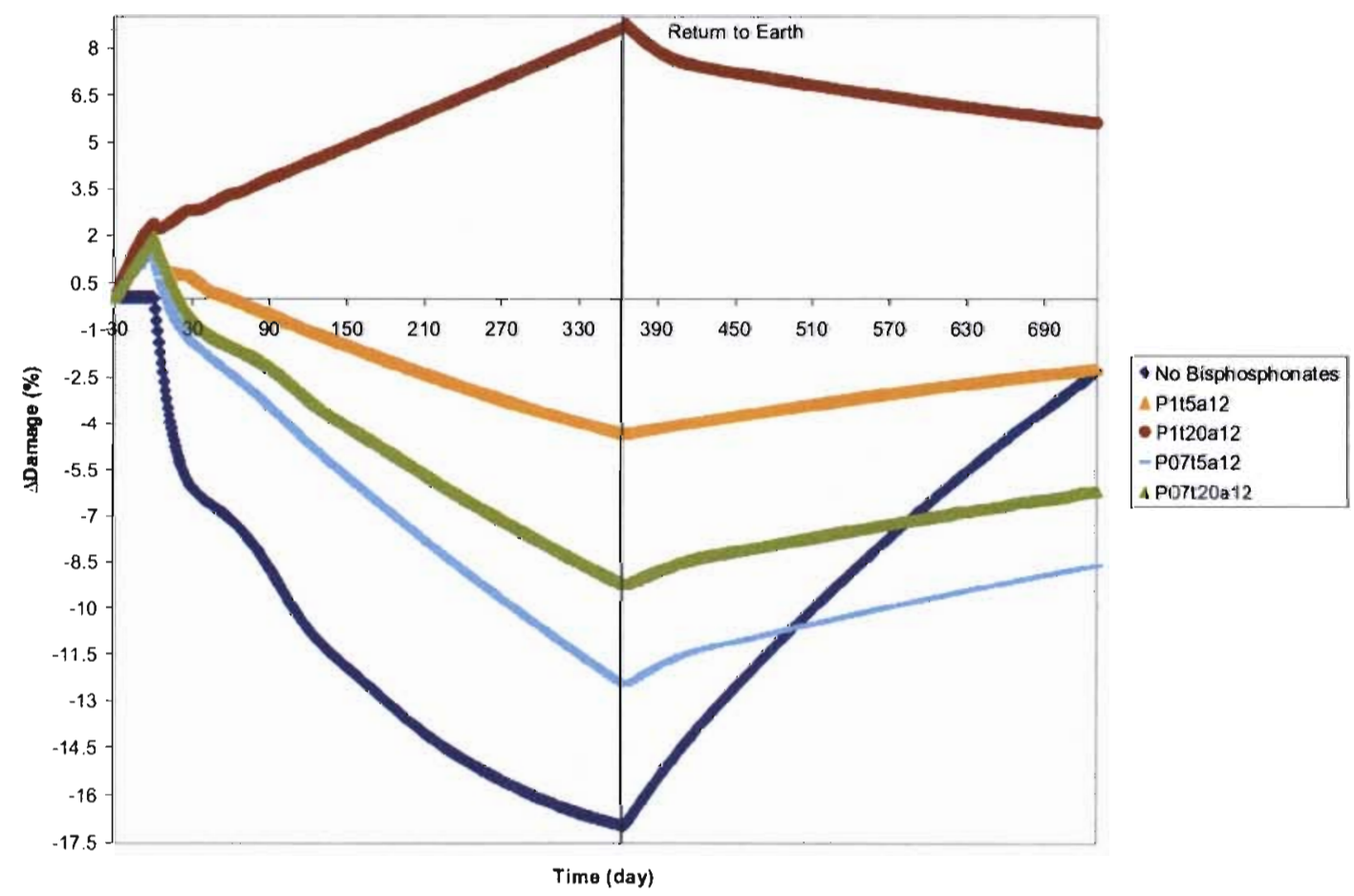

Figure E14. Predicted bisphosphonate effects beginning 30 days preflight on damage accumulation (D) and posttreatment return to Earth from 365-day spaceflight. 


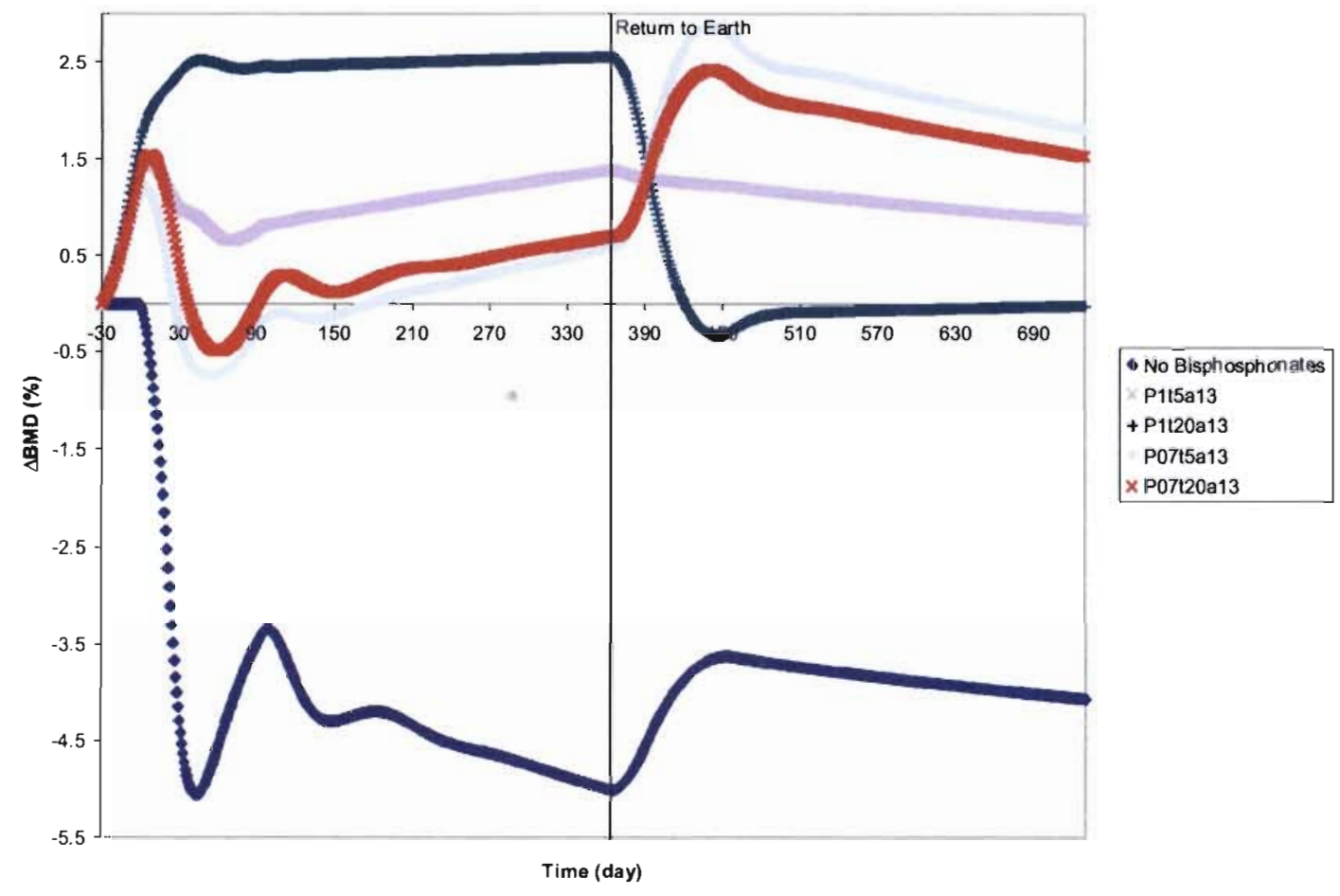

Figure E15. Predicted bisphosphonate effects beginning 30 days preflight on BMD and posttreatment return to Earth from 365 -day spaceflight.

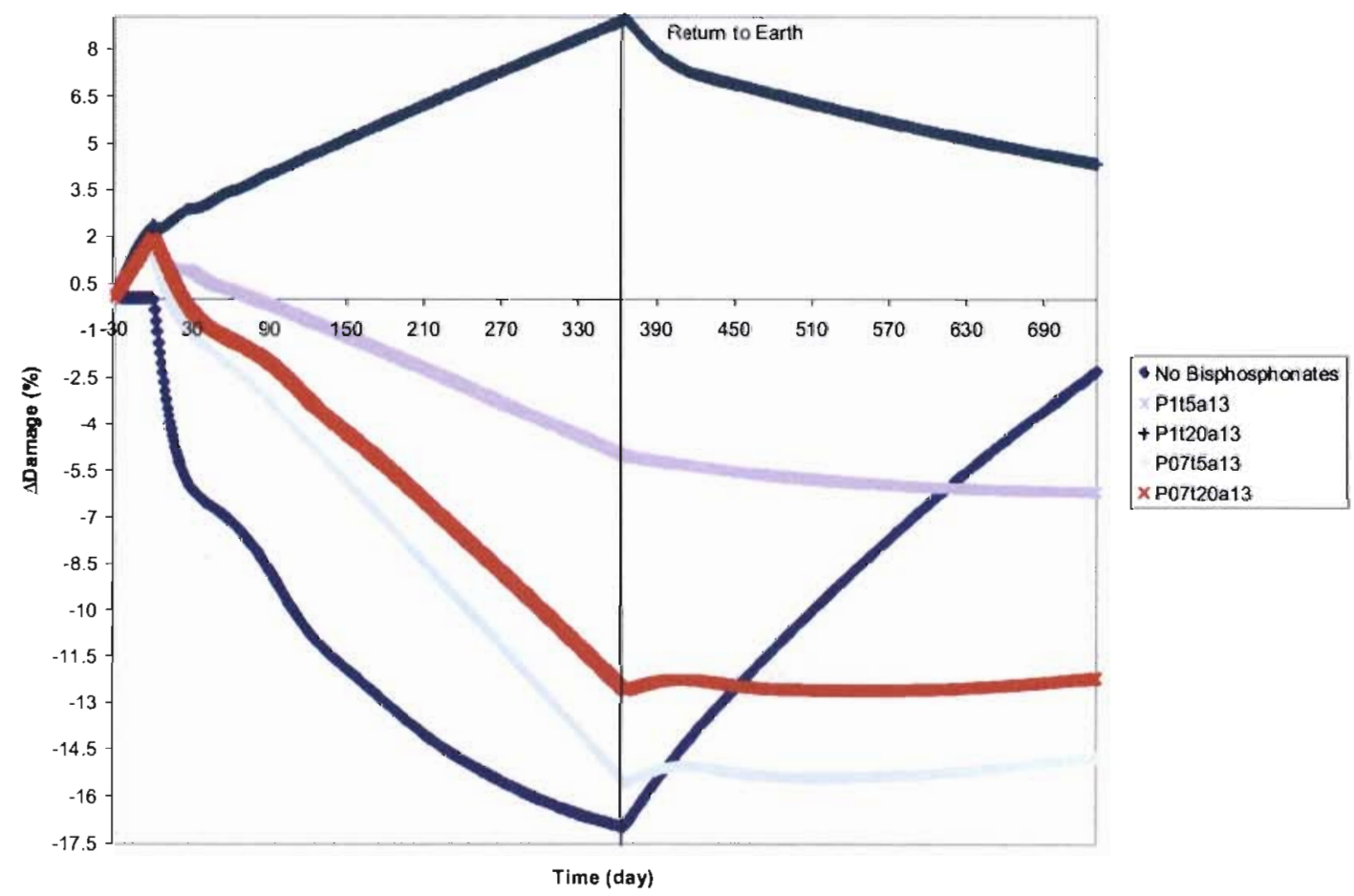

Figure E16. Predicted bisphosphonate effects beginning 30 days preflight on damage accumulation (D) and posttreatment return to Earth from 365-day spaceflight. 


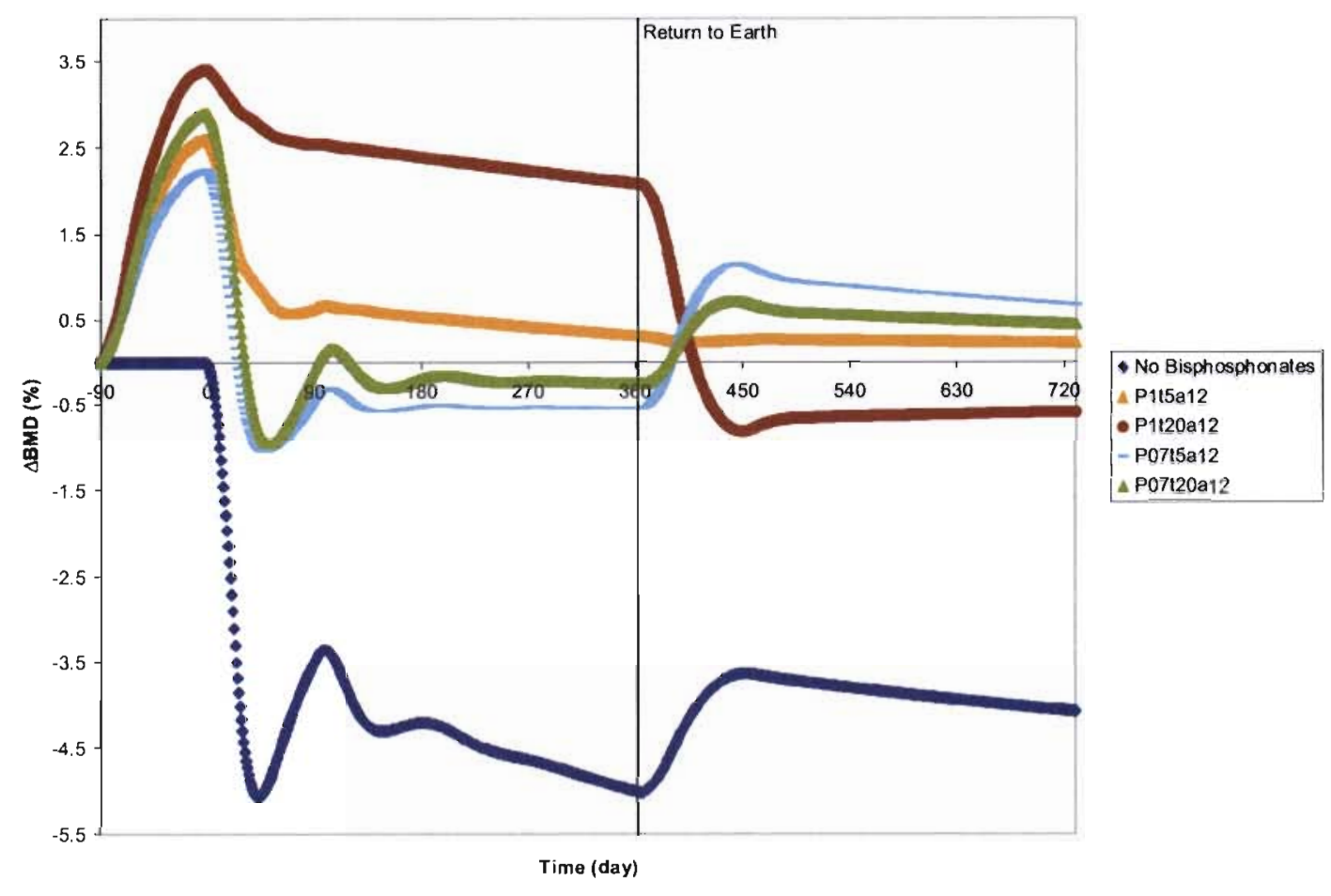

Figure E17. Predicted bisphosphonate effects beginning 90 days preflight on BMD and posttreatment return to Earth from 365-day spaceflight.

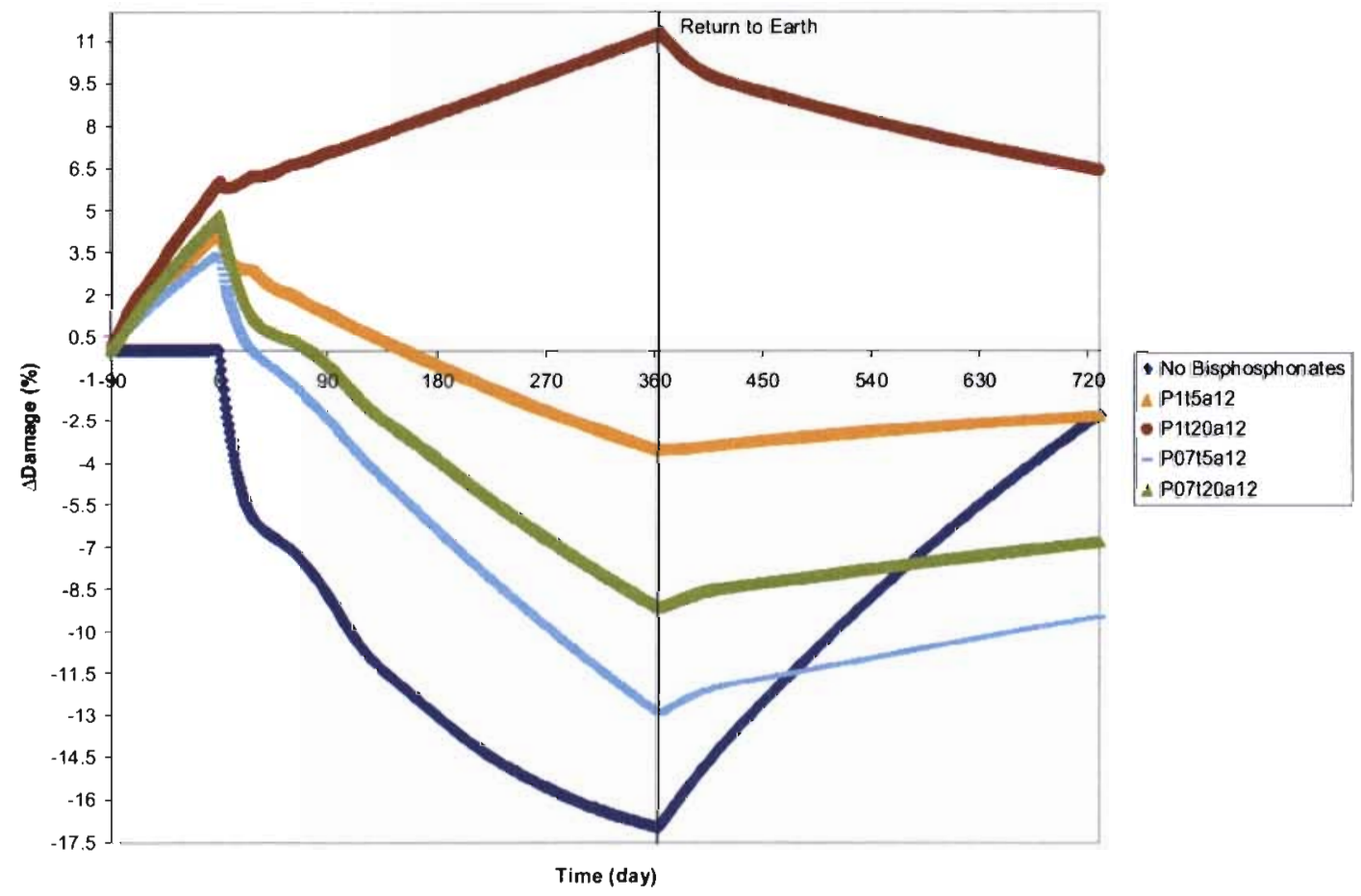

Figure E18. Predicted bisphosphonate effects beginning 90 days preflight on damage accumulation (D) and posttreatment return to Earth from 365-day spaceflight. 


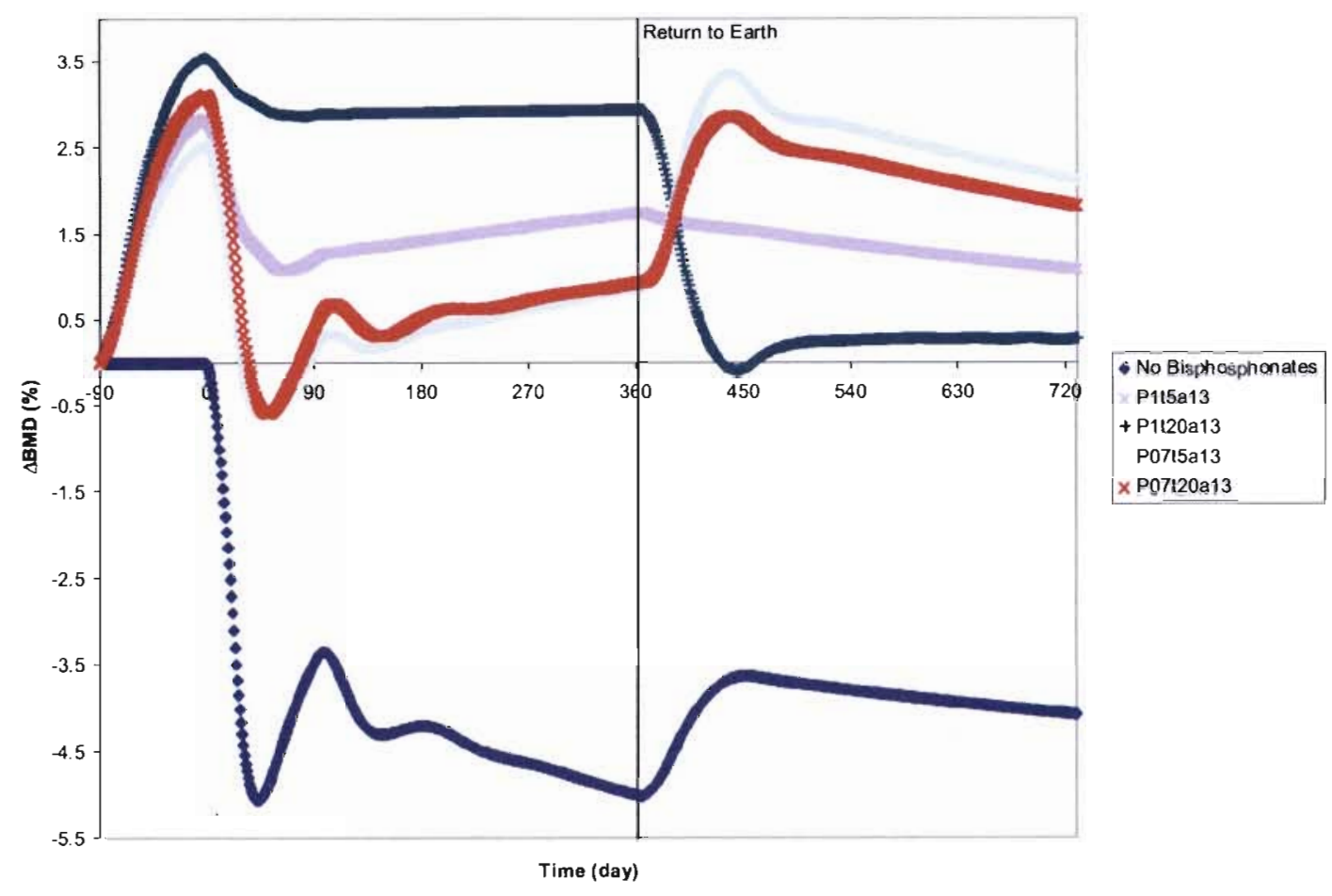

Figure E19. Predicted bisphosphonate effects beginning 90 days preflight on BMD and posttreatment return to Earth from 365-day spaceflight.

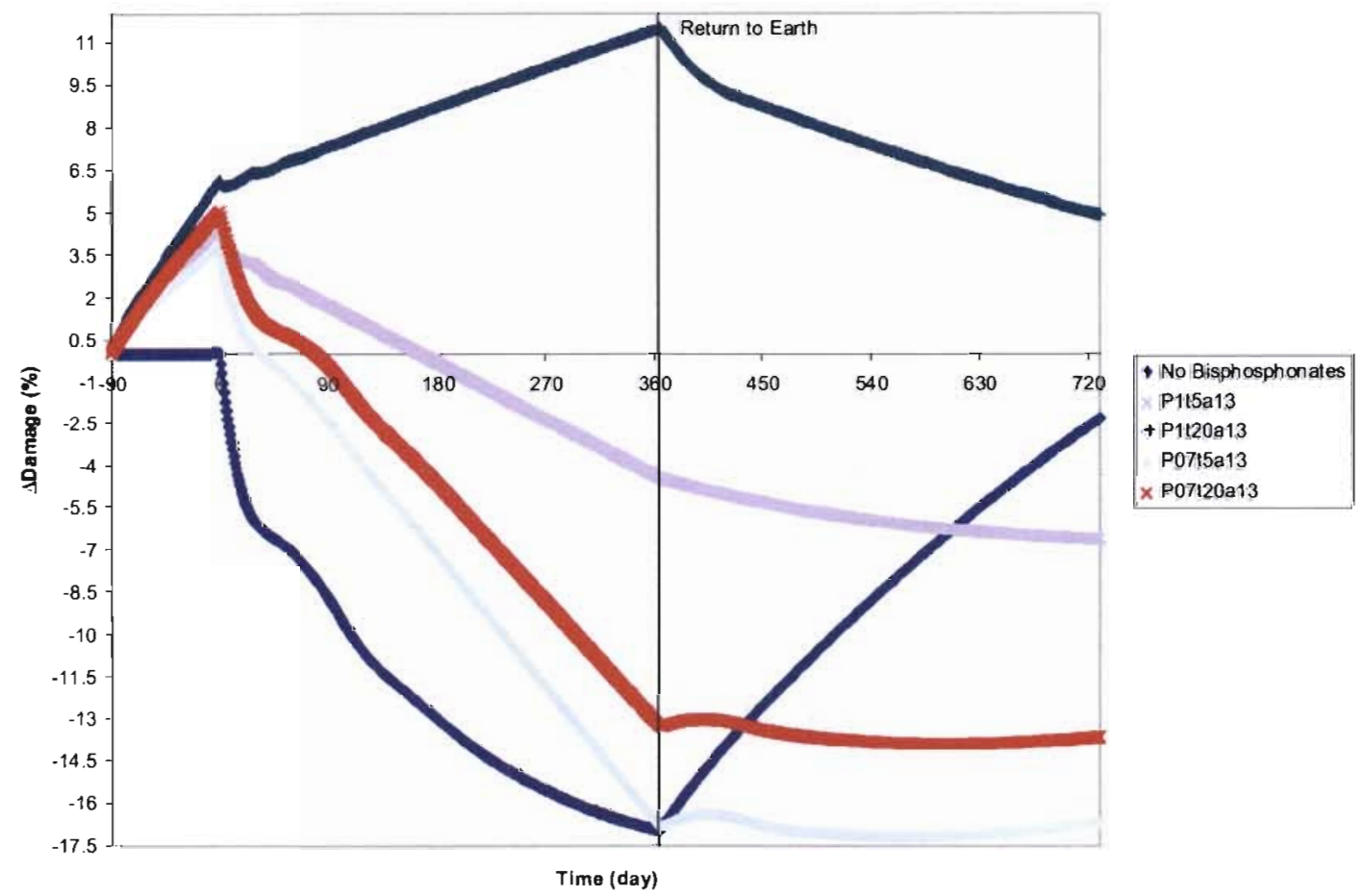

Figure E20. Predicted bisphosphonate effects beginning 90 days preflight on damage accumulation (D) and posttreatment return to Earth from 365-day spaceflight. 


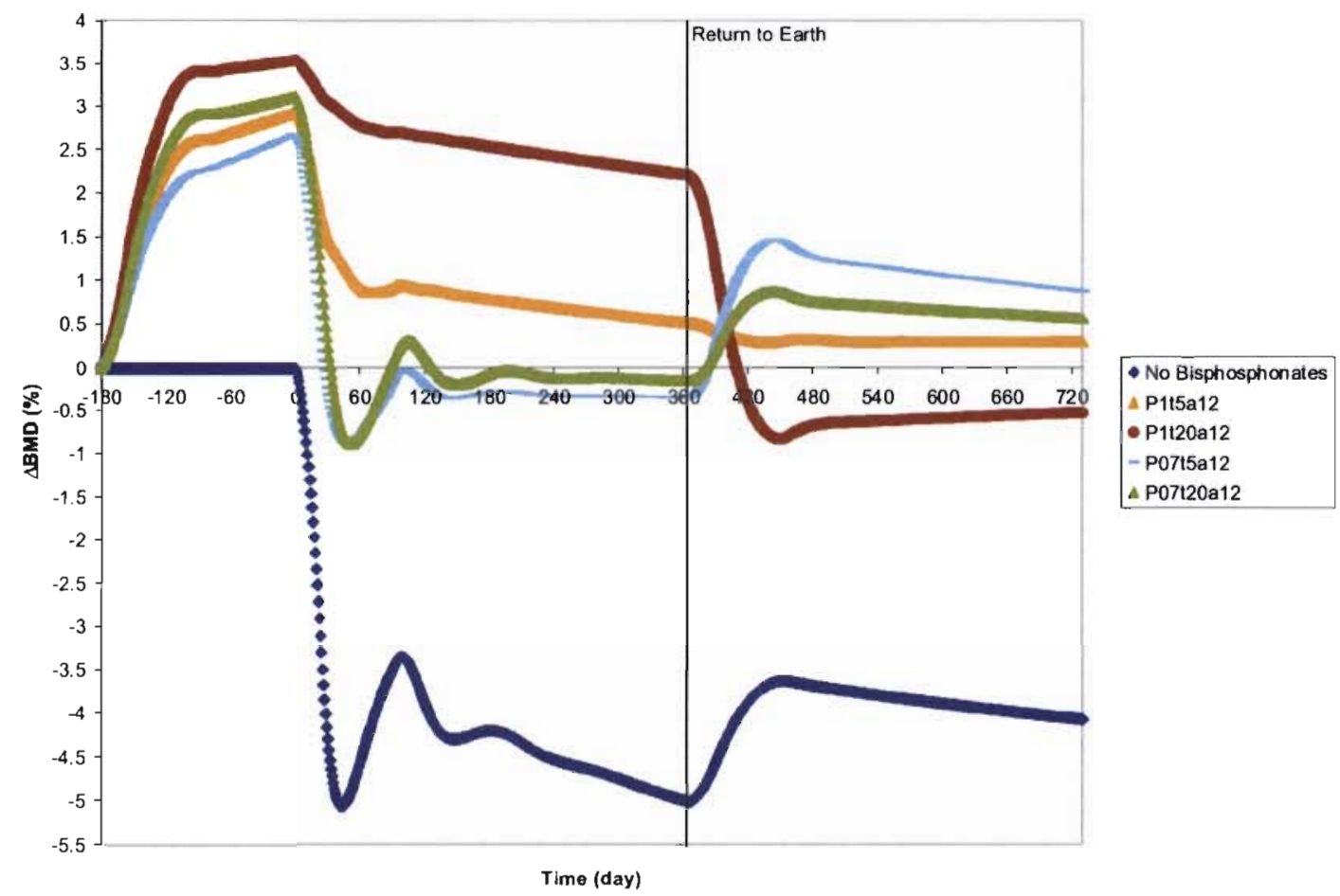

Figure E21. Predicted bisphosphonate effects beginning 180 days preflight on BMD and posttreatment return to Earth from 365-day spaceflight.

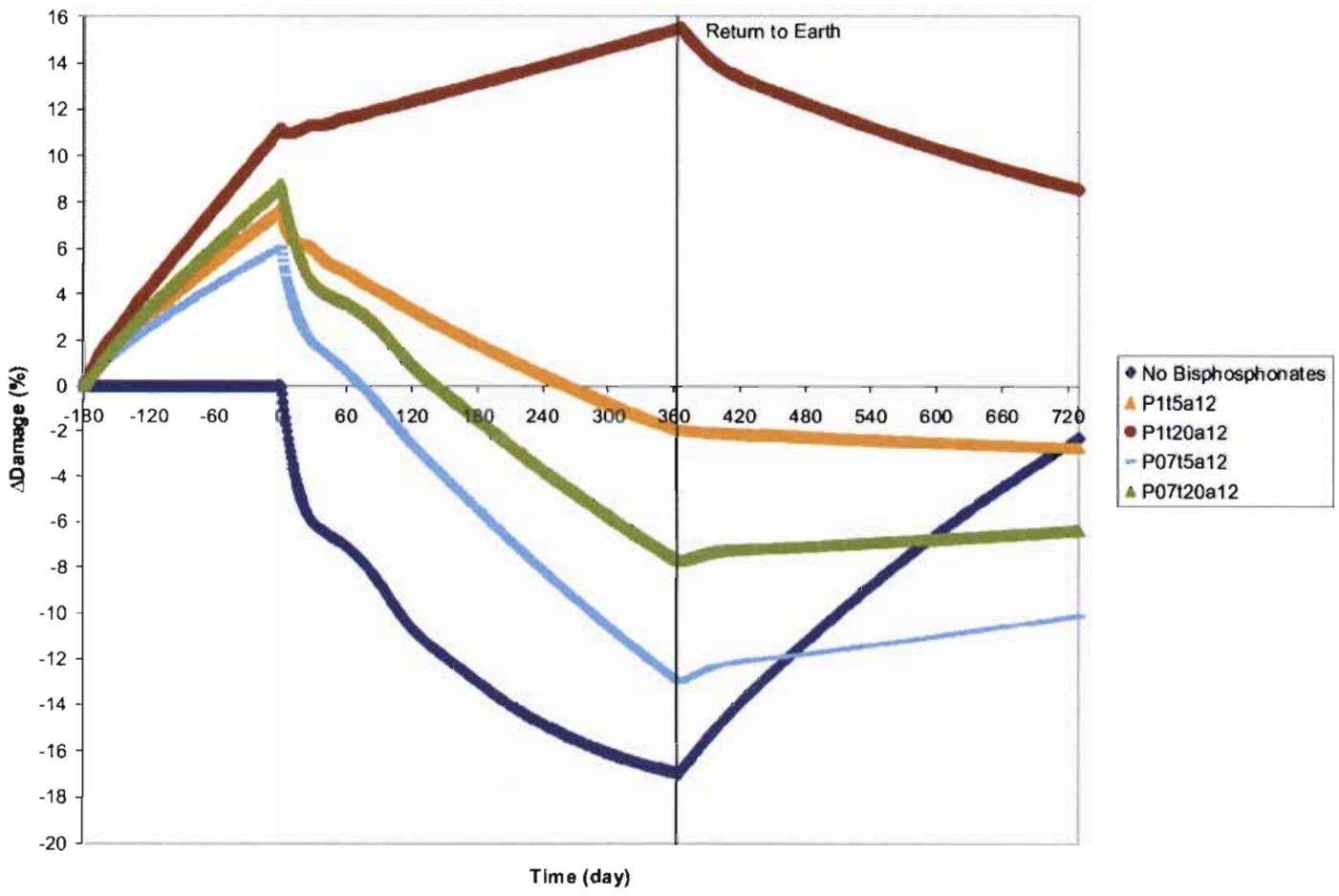

Figure E22. Predicted bisphosphonate effects beginning 180 days preflight on damage accumulation (D) and posttreatment return to Earth from 365-day spaceflight. 


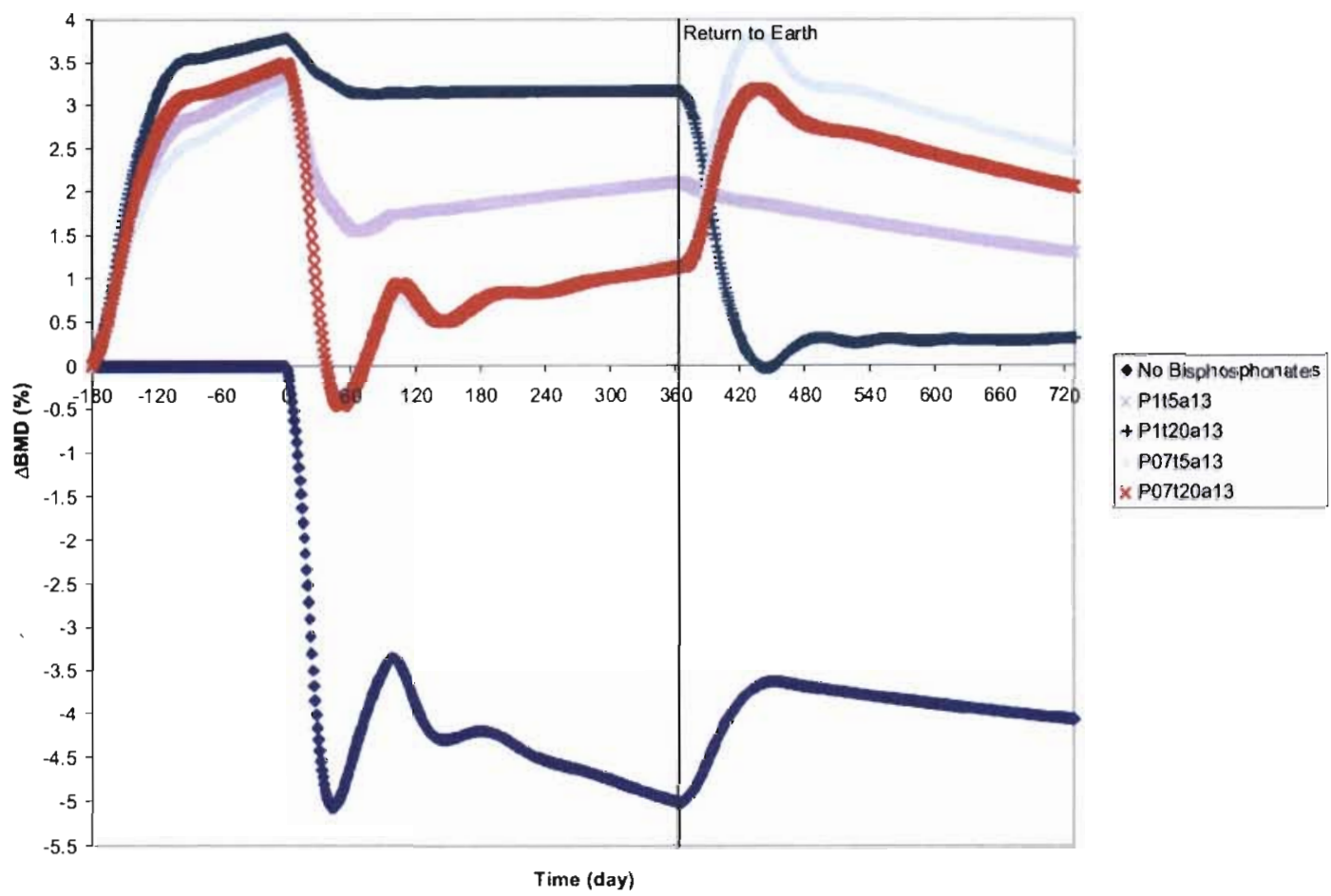

Figure E23. Predicted bisphosphonate effects beginning 180 days preflight on BMD and posttreatment return to Earth from 365-day spaceflight.

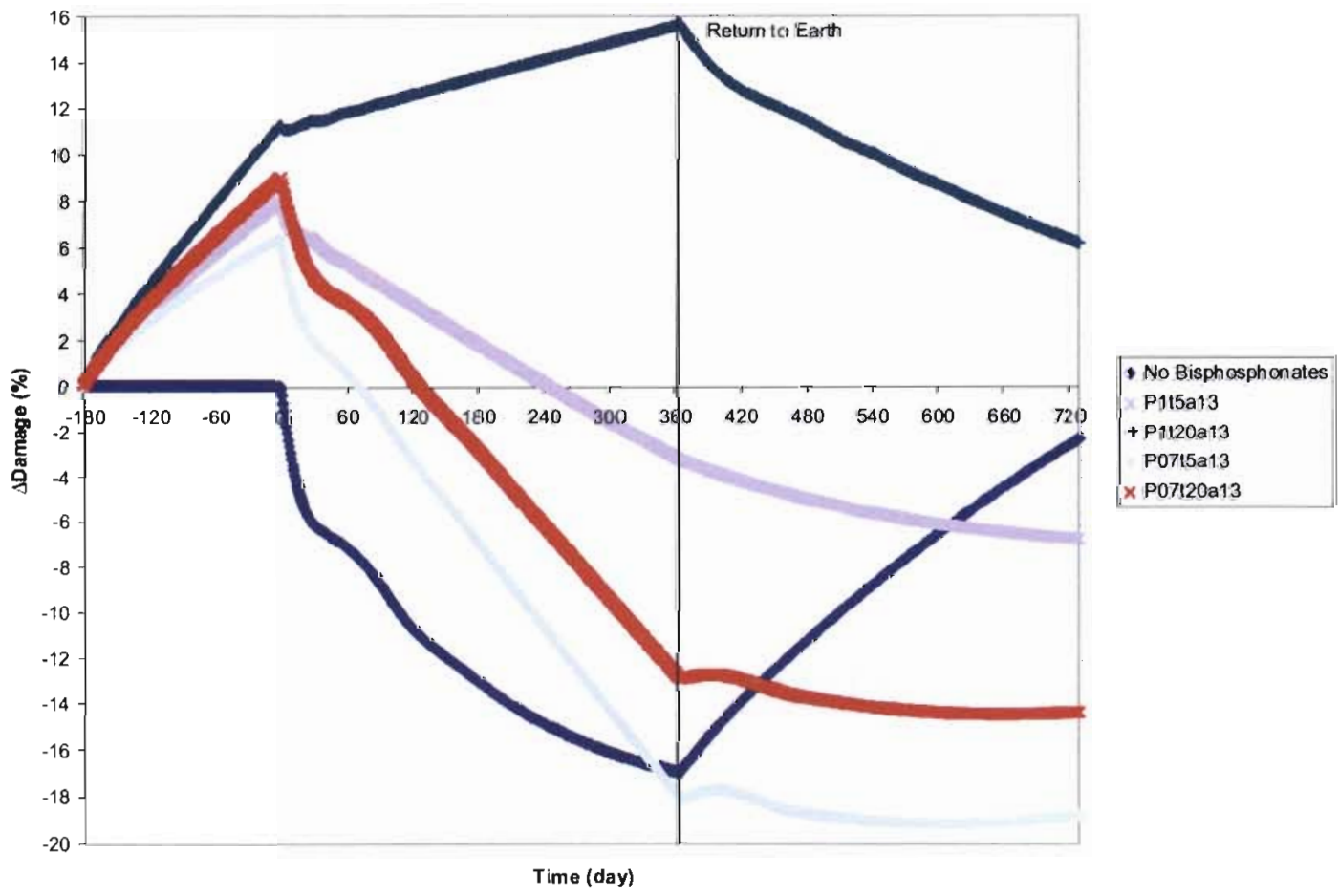

Figure E24. Predicted bisphosphonate effects beginning 180 days preflight on damage accumulation (D) and posttreatment return to Earth from 365-day spaceflight. 